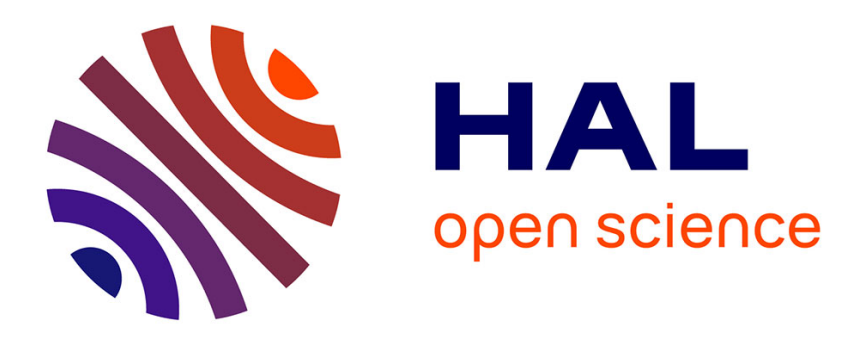

\title{
Bilan hydrochimique et géochimique du Lac Léman
}

Michel Meybeck

\section{To cite this version:}

Michel Meybeck. Bilan hydrochimique et géochimique du Lac Léman. Hydrologie. Université de Paris, 1971. Français. NNT: . tel-00822170

\section{HAL Id: tel-00822170 \\ https://theses.hal.science/tel-00822170}

Submitted on 14 May 2013

HAL is a multi-disciplinary open access archive for the deposit and dissemination of scientific research documents, whether they are published or not. The documents may come from teaching and research institutions in France or abroad, or from public or private research centers.
L'archive ouverte pluridisciplinaire HAL, est destinée au dépôt et à la diffusion de documents scientifiques de niveau recherche, publiés ou non, émanant des établissements d'enseignement et de recherche français ou étrangers, des laboratoires publics ou privés. 


\section{BILAN HYDROCHIMIQUE ET \\ GEOCHIMIQUE DU LAC LEMAN}

\section{par Michel Meybeck}

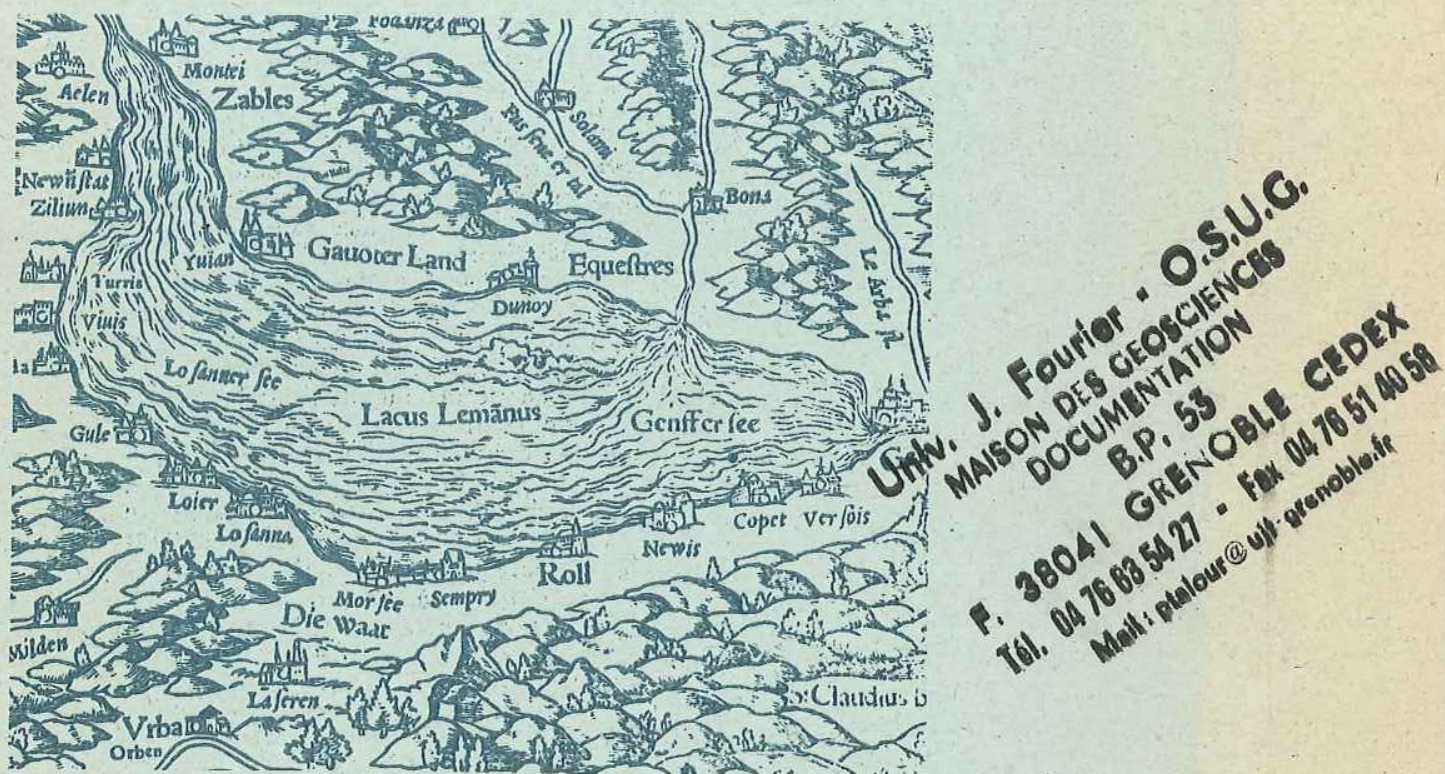

CENTRE DE RECHERCHES GEODYNAMIQUES

THONON-LES - BAINS 74 FRANCE 
LA FACULTE DES SCIENCES DE PARIS

pour 1'obtention du

DOCTORAT DE 3ème CYCLE

SPECIALITE GEODYNAMIQUE EXTERNE

par Miche1 MEYBECK

BILAN HYDROCHIMIQUE et GEOCHIMIQUE DU LAC LEMAN

Soutenue 1e : 9.01 .1971

devant la Commission composée de

Président
Examinateurs

L. Glangeaud

Examinateurs

P. Bellair

$\mathrm{Ph}$. Olive

Invité

A. Burger

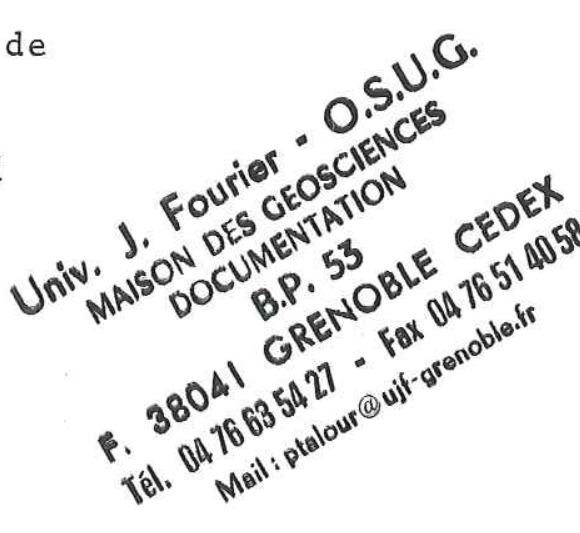

CENTRE DE RECHERCHES GEODYNAMIQUES

$$
74 \text { - THONON (FRANCE) }
$$


Au moment de soumettre mon travail à l'apprêciation du jury, c'est un agréable devoîr d'adresser ici mes sincères remerciements à tous ceux quî m'ont aîdê.

- A Monsieur 1e Professeur Louis Glangeaud, Membre de 1 'Institut, pour la confiance qu'îl $\mathrm{m}^{\prime a}$ tểmoìgnée en m'acceptant comme Assistant dans son Laboratoire et pour I'honneur qu'il me fait en présidant ce jury.

- A Monsieur le Professeur Pierre Bellair qui a bien voulu examiner et critiquer ce travail.

- A Monsieur le Professeur Andrê Burger, spécialement venu de Neuchate1, quỉ représente à ce jury le pays quí est le berceau de la limnologie.

- A Monsieur Philippe 0live, Sous-Directeur du Centre de Recherches Géodynamîques de Thonon, quỉ m'a accueilli, ill y a trois ans, dans I'équipe du C.R.G. Là j'aì pu bénéficier des meilleures conditions de travail, et les nombreuses études que nous avons effectuées en équipe m'ont beaucoup enrichí.

Ce travail n'aurait pas été possible sans le concours de nombreuses personnes. L'ensemble des prélèvements sur le lac a étê réalisé avec Monsieur G. Olivier, toujours avec compétence et gentillesse, je n'oublierai pas toutes ces sorties sur le Lëman et ce travail lui doit beaucoup. J'ai ëgalement une grande dette envers Monsieur P. Blanc et Madame A. Noir qui ont effectué avec beaucoup de rigueur l'ensemble des analyses chîmiques qui sont à la base d'une grande partìe de cette étude. 
J'aî aussî bênếficiciê du concours de Madame S. Chessel pour les analyses de tritium et de Mesdames $O$. Contamine et L. Ferrari pour les nombreuses filltrations et analyses physiques des sédiments, à toutes trois $j$ 'adresse mes vifs remerciements.

La réalisation technique de ce travaîl a étê assurée avec beaucoup de soin par Mademoîselle M. Lecraz et Madame Duchamp pour la frappe, Mademoiselie A.M. Chiara pour la mise en page et le tirage et Monsieur R. Carrier pour les dessins, qu'ills trouvent îcî l'expression de ma sincère reconnaissance.

Mes remerciements vont également à :

- Messieurs C. Bobier et J. Petit de la Station de Gêodynamique sous-marine de Villefranche-sur-Mer qui ont rendu possible la campagne de sismique continue.

- Messieurs Guenin, Kolher et Fischer de I'Office Fêdéral de I'Economie Hydraulique de Berne pour les documents très utiles qu'ils ont mis à ma disposition.

- Messieurs Burkard et Monod de la Commission Internationale pour la Protection des Eaux du Léman et du Rhône contre la Pollution pour les renseignements précieux que j'ai trouvề dans leurs rapports.

Tout au long de ce travail, j'ai pu bénéficier des conseils de mes camarades du Centre Géodynamîque, Mademoiselle E. Crouzet, Messieurs M. Bakalowicz, J.M. Martin, E. Siwertz et en particulier P. Hubert avec lequel j'ai eu le plaisir de partager ce magnifíque terrain de thèse qu'est le Léman. Je n'oublierai pas cette amicale collaboration.

Enfin que ceux qui, par leur amitié et leur affection, m'ont soutenu tout au long de ce travail, trouvent icì l'expression de ma profonde gratitude.

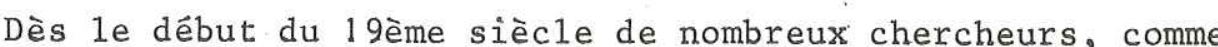
De Saussure, se sont intéressê au Léman, mais ce fut Forel qui, à partir de 1880, mena à bien la première étude complète du Léman. On peut même dire que son travail fut à la base de la science des lacs : 1a limnologie. Ce savant encyclopédiste entreprit de vastes recherches allant de la physico-chimie des eaux à l'histoire des populations riveraines en passant par la sédimentologie, la biologie, etc... Ces travaux sont essentiels pour deux raisons : tout d'abord c'est la première étude moderne d'un $1 \mathrm{ac}$, ensuite Forel nous donne l'état du Léman vers 1880 , tout particulièrement en ce qui concerne la qualité des eaux, le cycle du plancton, la faune et la flore. Il manquait cependan une carte bathymétrique précise des fonds du Léman. Elle fut réalisée de 1886 à 1889 par Hörnlimann pour 1a partie suisse et Delebecque pour la partie française. Les éléments de base étant réunis, de nombreux chercheurs ont poursuivi les recherches de Forel. Citons notamment, avant 1945, Gagnebin, Parejas, Romieux,pour la géologie et la sédimentologie, Kreitmann et Mercanton pour 1 'étude des courants. Après 1945 les études se sont très diversifiées, citons Dussart pour 1'hydrologie, Juget pour la biologie, Monod pour la chimie, Novel pour la bactériologie. Ces dernières années le Léman a été étudiê au Centre de Recherches Géodynamiques (Glangeaud, Serruya, Olive, Sauvage, VergnaudGrazzini, Hubert, Meybeck, Fontes,...) par des méthodes nouvelles, comme la stratigraphie des sédiments superficiels, la sismique continue, les isotopes, la palynologie.

L'accès aisé du Léman, la proximité de nombreux Centres de Recherches ou Universités ont beaucoup favorisé ces recherches. Il se dessine actuellement des regroupements des chercheurs des diffêrentes 
disciplines. D'une part la "Commission Franco-Suisse de Protection du Léman et du Rhône contre la Pollution" groupe tous les laboratoires travaillant en hydrochimie et biologie. (Laboratoires cantonaux des cantons de Genève, de Vaud et du Valaîs, Station d'Hydrobiologie Lacustre de $1^{\prime} I \circ N \circ R$.A. à Thonon, et Centre de Recherches Géodynamiques de Thonon). Ses travaux de contrôle de la qualité des eaux du lac sont très complets et nous ont servì plusieurs fois. D'autre part il s'est constituê, il y a deux ans, un groupe d'étude géologique du Léman, Geolem, constitué par le Bureau de Recherches Géologiques et Minières, I'Universitể de Genève et le Centre de Recherches Géodynamiques de Thonon. Ce groupe travaille essentiellement sur la structure du Léman et la géochimie des sédiments avec application à l'étude de la pollution des sédiments, phénomène non considéré jusqu'à présent.

On pouvait donc considérer que le Léman était un lac parfaitement connu. Mais en fait de nombreux points restaient encore imparfaitement étudiés, notamment en ce qui concerne la chimie des eaux et des sédiments du Léman, ainsi que la structure de la cuvette lémanique. De plus, jusqu'ici aucun bilan global du bassin versant du Léman n'avait étê entrepris. Un tel bìlan hydrochimique, sêdimentaire et géochimique est important pour trois raisons :

- le Léman est situé dans un environnement climatique, géologique et géographique qui détermine ses caractères généraux. L'étude des apports permettra donc de connaître la nature des bassins versants et de leur relation avec le lac.

- à 1'heure où les problèmes d'eutrophisations et de polZutions se posent avec acuitê, l'êtablissement des bilans des lacs est un problème essentiel mais très délicat à mettre en oeuvre.

- enfin du point de vue de la géodynamique externe le bilan $d^{\prime}$ un bassin versant de $8000 \mathrm{~km}^{2}$ donne des indications précieuses sur les phénomènes actuels d'érosion et de transport. En ajoutant à ces données celles obtenues pour le Bodensee, nous obtiendrons des résultats concernant toute cette partie des Alpes centrales.

Notre étude a duré seulement trois ans. Pour un lac comme le Léman, dont le temps de renouvellement des eaux est de I'ordre de io ans, cette période est encore insuffìsante. Aussi, nous avons été amené à tenir le plus grand compte des échelles de temps et d'espace des phénomènes principaux, afin de présenter un bilan aussi représentatif que possible. 


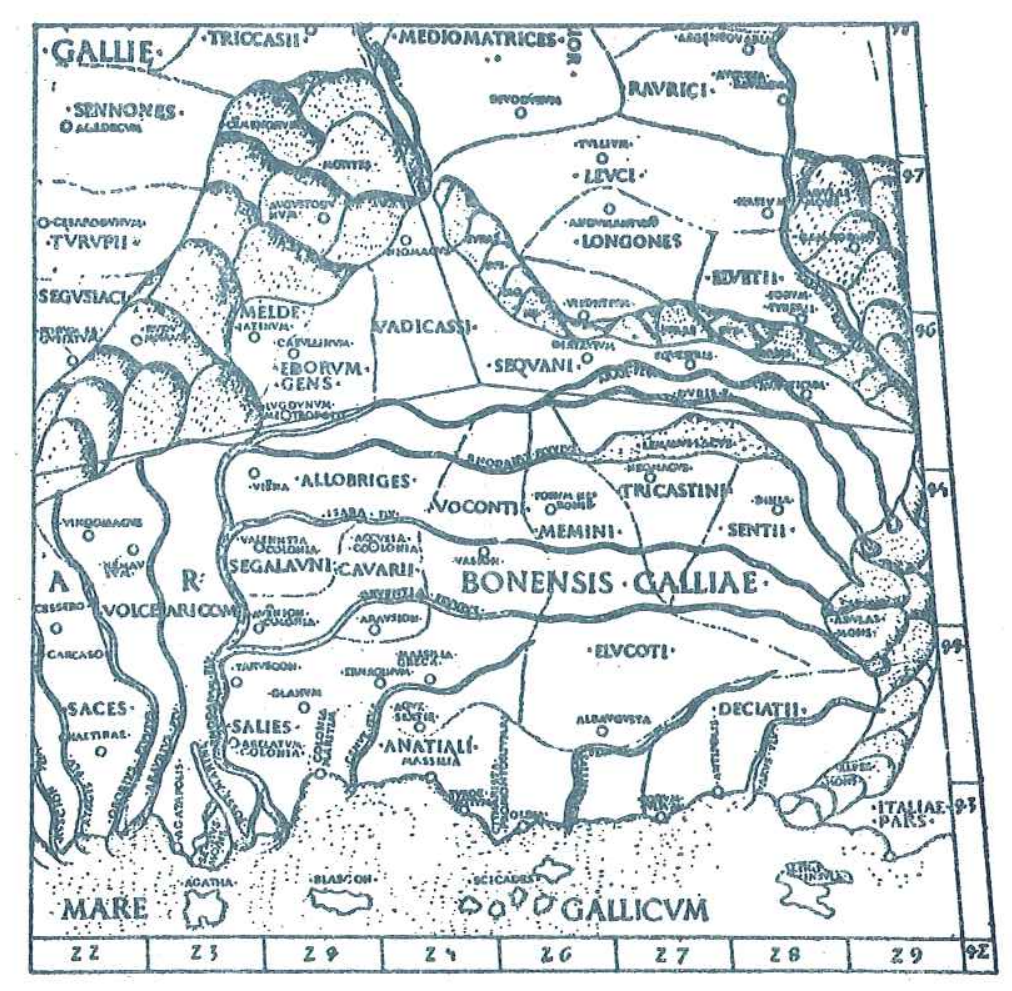




\section{1. - Données morphométriques.}

L'arc Alpin est jalonné de nombreux lacs qui ont en commun leur grande dimension et une origine entièrement ou en partie glaciaire (figure $I_{1}$ ), parmi eux se trouve le Léman. C'est un des plus grands lacs d'Europe occidentale : il est le premier pour le volume des eaux, le quatrième pour la profondeur maximale (après le lac de Come - 410 m -, 1e 1ac Majeur - 372 m - et 1e lac de Garde - 346 m). I1 est souvent divisé en deux parties : 1e Grand Lac qui occupe la cuvette centrale et le Petit Lac ou Lac de Genève qui est la partie occidentale étroite et peu profonde. Les caractéristiques du Léman sont résumées dans le tableau suivant (d'après Forel, 1895 et Delebecque, 1898).

TABLEAU $I_{1}:$ Caractêristiques morphométriques du Léman

\begin{tabular}{|l|l|l|l|}
\hline & Léman & Grand Lac & Petit Lac \\
\hline Altitude du plan d'eau & $372 \mathrm{~m}$ & \\
\hline Volume (V) & $89 \mathrm{~km}^{3}$ & $85,8 \mathrm{~km}^{3}$ & $3,2 \mathrm{~km}^{3}$ \\
\hline Surface (S) & $582 \mathrm{~km}^{2}$ & $503 \mathrm{~km}^{2}$ & $79 \mathrm{~km}^{2}$ \\
\hline Profondeur maximale & $309 \mathrm{~m}$ & $309 \mathrm{~m}$ & $76 \mathrm{~m}$ \\
\hline Profondeur moyenne (V/S) & $152 \mathrm{~m}$ & $172 \mathrm{~m}$ & $41 \mathrm{~m}$ \\
\hline Longueur & $72 \mathrm{~km}$ & $49 \mathrm{~km}$ & $23 \mathrm{~km}$ \\
\hline
\end{tabular}




\section{2. - Hydrologie.}

L'ensemble du bassin versant du Léman couvre une surface de $7975 \mathrm{~km}^{2}$, son altitude moyenne est de $1670 \mathrm{~m}$ (figure $\mathrm{I}_{2}$ ). Le principal affluent est le Rhône Alpestre qui provient du Valais, à lui seul il représente $70 \%$ des apports d'eau au lac. La Dranse du Chablais est le deuxìème affluent avec $10 \%$ des apports, le reste des apports est constitué par. une vingtaine de petits affluents parmi lesquels ìl convient de cîter la Venoge. L'exutoỉe du Léman, régularisé par des vannes, est à Genève.

*1.3. - Bathymétrie.

Le centre du Grand Lac est caractérisé par une vaste étendue plane de $12 \mathrm{~km}$ sur $6 \mathrm{~km}$, d'une profondeur de 307 à $309 \mathrm{~m}$, elle est gểéralement appelée plaine centrale. Les fonds entre la plaine centrale et l'embouchure du Rhône correspondent au deltra sous-

lacustre de cette rivière. Vers 1 'ouest les fonds remontent régulièrement jusqu'à la barre de Promenthoux (61 m de profondeur) qui sépare le Grand Lac du Petit Lac (figure $I_{3}$ ). Des coupes transversales montrent bien trois types de profils : le Petít Lac présente des profils semi-ovalaires, les pentes sont régulières jusqu'au milieu du profil, dans la partie occidentale du Grand Lac le profil devient triangulaire, par contre dans la partie orientale il est nettement trapézoïdal : les pentes sont abruptes et les fonds presque horizontaux. Une caractéristique importante des fonds du Léman est la présence d'une échancrure dans le delta sous-lacustre du Rhône, la carte de Delebecque-Hörnlimann (Delebecque 1898) montre très bien cette particularitê sur laquelle nous reviendrons. Nous avons déjà signalé le delta du Rhône, d'autres deltas sous-lacustres sont visibles : le principal est celui de la Dranse du Chablais, citons aussi celui de la Venoge. Enfin signalons quelques parties peu profondes du lac comme les baies d'Excenevex et de Villeneuve. 

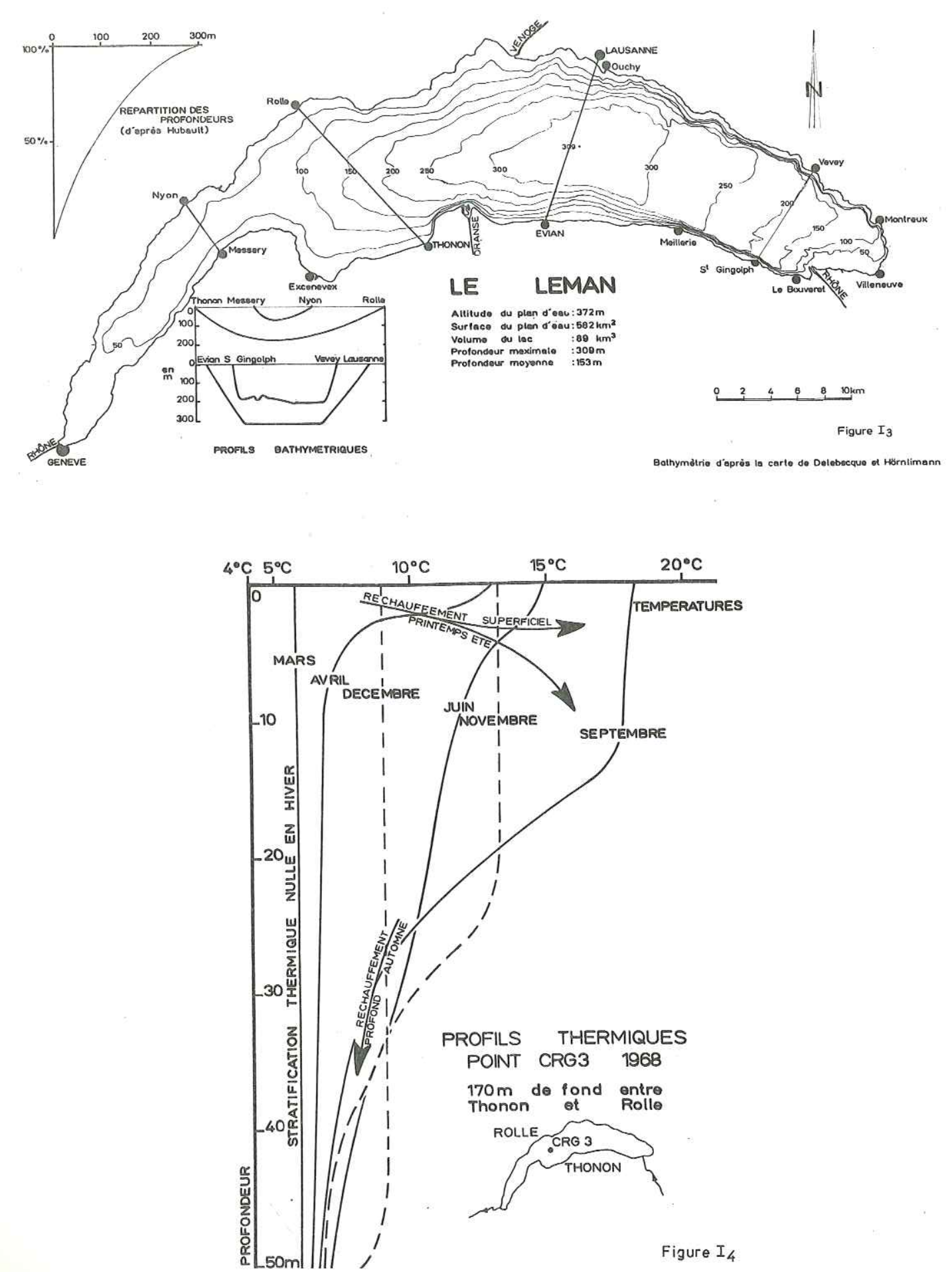

1.4. - Thermique et chimie des eaux.

La thermîque du Léman est bien connue depuis Forel, ce dernier I'avait classé dans les lacs "tropicaux", Dussart en fait un Iac "monomîctique chaud de premier ordre". Le Léman a toujours en effet une température supérieure à $4^{\circ} \mathrm{C}$, et les eaux profondes ont une température voîsine de $4^{\circ} \mathrm{C}$ pendant toute 1 'annếe. I1 comprend une période de cîrculation en hiver et une période" de stratification en étê. En effet l'énorme volume du Léman et sa grande profondeur font que le refroidissement hivernal n'est jamais assez intense pour refroìdir toute la masse en dessous de $4^{\circ} \mathrm{C}$ (maximum de densité de $1^{\prime}$ eau pure). Les rares congélations du Léman sont reportées pour la rade de Genève peu profonde. La figure $I_{4}$ montre bien les variations de température $\mathrm{du}$ Lac et le processus de réchauffement. La thermocline qui représente la profondeur du lac où le gradient de température est le plus élevé (point d'inflexion des courbes températures-profondeur) commence à apparaître en mars-avril en surface, s'abaisse progressivement jusqu'à $50 \mathrm{~m}$ en novembre-décembre, puis s'atténue complètement de décembre en mars. La thermocline est très accusée en période de stratification thermique de juillet en septembre, elle se situe alors entre 15 et $20 \mathrm{~m}$, le lac est ainsi nettement séparé en deux parties entre lesquelles les échanges sont très difficiles : l'épilimnion entre la surface et la thermocline et 1 'hypolimnion entre la thermocline et le fond.

Le Lëman est peu minéralisé : environ $200 \mathrm{mg} / 1$ d'éléments dissous, les éléments príncipaux sont les bicarbonates, les sulfates, le calcium et le magnêsium. Nous reviendrons plus en détail sur la chimie des eaux dans le chapitre correspondant.

\section{5. - Géologie.}

Le Léman est situé dans la dépression molassique entre les ensembles alpins et jurassiens : au Sud les nappes des prêalpes du Chablais et du Valais, et au Nord 1a Haute Chaîne du Jura dont les 

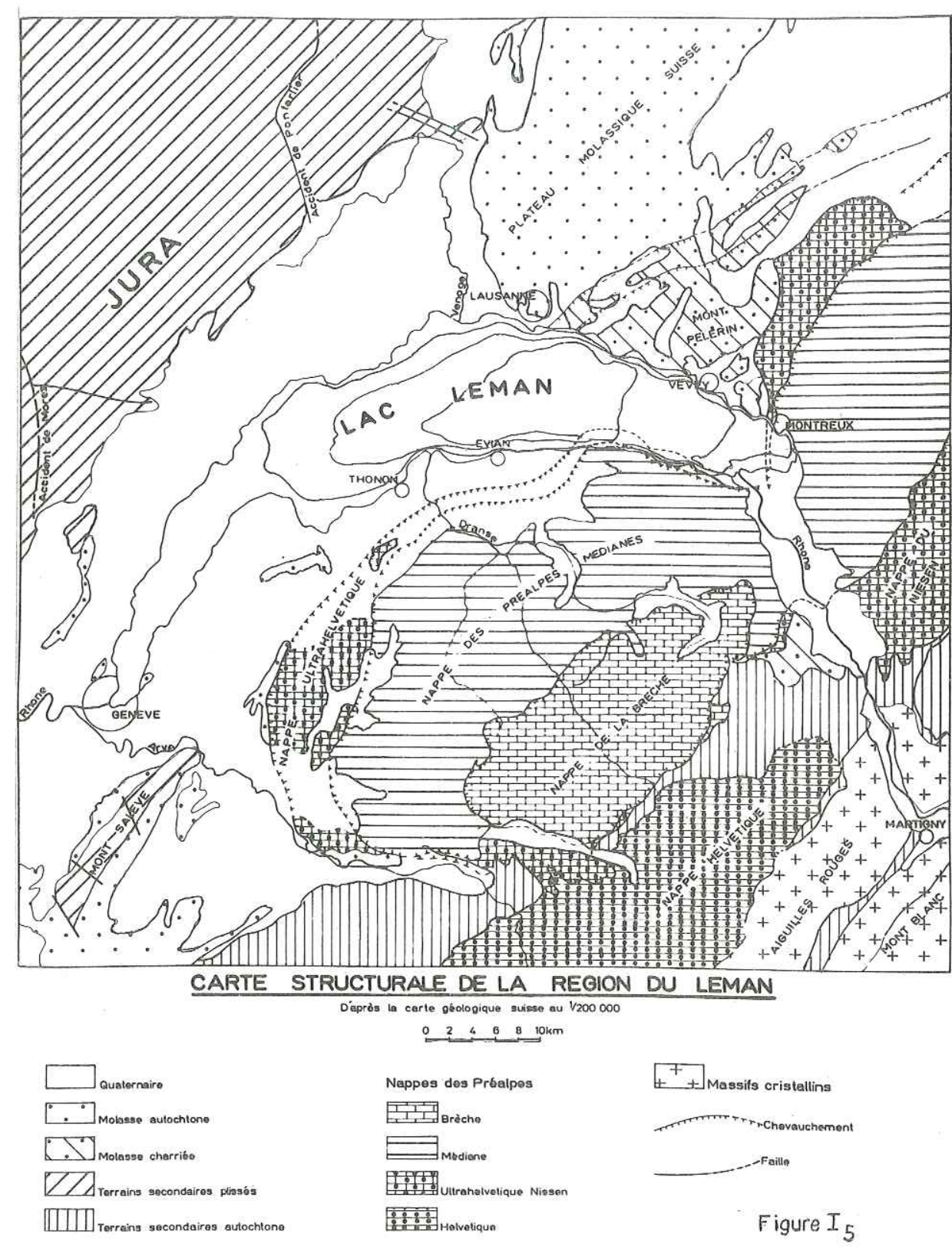

plis sont soumîs à de nombreux décrochements (Pontarifer, Morez). Le Lac occupe donc une positîn particulièrement importante entre ces deux domaìnes. La figure Is reprèsente la structure du Bassin Lêmanique d'après la carte gểologique Suísse au 1/200 000. Depuis, un grand nombre de données nouvelles ont étế apportées, en particulier par les études de sismàque continue sur le lac. Ces problèmes seront discutếs dans le cinquième chapitre.

\section{6. - Climat de la région du Léman.}

B. Blavoux et al (1962) ont défini les caractères climatiques principaux de la région du Léman ainsì : la température moyenne annuelle de $1^{\prime}$ air est de $9^{\circ} 38$ à Genève. La température moyenne des eaux de surface est de $1^{\circ} 85$ supérieure à celle de $1^{\prime}$ ai à Thonon, le lac est ainsi responsable d'un microclimat; par exemple l'amplitude annuelle de la tempêrature de $1^{\prime}$ air à Thonon est de $18^{\circ} \mathrm{C}$ soít $2^{\circ} \mathrm{C}$ inférieure à celle qu'on pourrait atteindre si le Léman n'existait pas. L'humîdîtê relatîve de $1^{\prime}$ aìr est en moyenne de $75 \%$. La pluviosité sur le plan d'eau est environ de $1000 \mathrm{~mm} / a n$, et l'êvaporation est estimée entre $650 \mathrm{~mm} / \mathrm{an}$ et $850 \mathrm{~mm} / \mathrm{an}$.

\section{7. - Comparaison du Léman avec le Bodensee.}

11 nous a semblé intéressant de comparer le Lếman au Bodensee dont les caractéristiques sont analogues (Dussart 1963):

\begin{tabular}{|l|l|l|l|}
\hline & Bodensee & Obersee & Untersee \\
\hline Surface & $539 \mathrm{~km}^{2}$ & $476 \mathrm{~km}^{2}$ & $63 \mathrm{~km}^{2}$ \\
\hline Volume & $48 \mathrm{~km}^{3}$ & 47,6 & 0,84 \\
\hline Altitude du plan d'eau & $395 \mathrm{~m}$ & & \\
\hline Profondeur maximale & $252 \mathrm{~m}$ & 252 & 46 \\
\hline Profondeur moyenne & $92 \mathrm{~m}$ & \\
\hline
\end{tabular}


Le Bodensee peut être divisé en deux bassins distincts : l'Obersee et 1'Untersee. L'Obersee se déverse dans 1 'Untersee par le Rhin lacustre (Seerhein) au niveau de la vîlle de Constance qui sépare les deux 1acs.

Les analogies entre le Bodensee et le Lếman ne s'arrêtent pas aux données morphométriques, nous verrons que ces deux lacs ont des situations géographiques, hydrologiques et géologiques comparables. Le Bodensee et son bassin versant étant très bien connus notamment après les travaux de Müller $(1964,1967,1968 \ldots$..), Waibel (1962) et de 1a Commission Internationale d'Etude du Bodensee. Nous pourrons donc les étudier parallèlement (chimie, sédimentologie, structure, etc...). Comme ces deux lacs jouent le rôle d'intégrateur de leurs bassins versants, il sera possible d'obtenir des données concernant les taux d'érosions sur l'ensemble des Alpes Suisses.

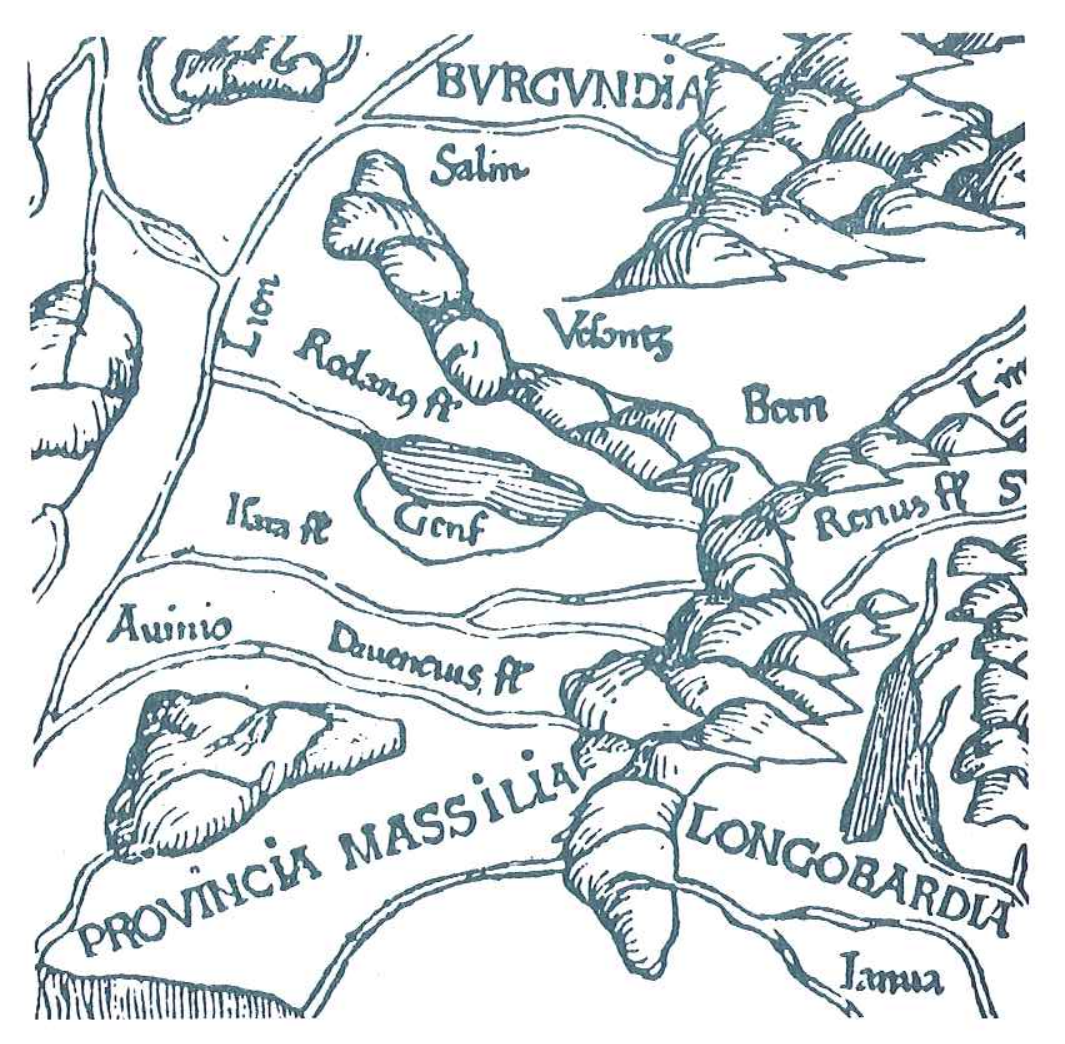




\section{1. - Caractères de I'étude chimique des affluents du Léman.}

Nous avons étudié les deux affluents principaux du Léman (figure $\mathrm{I}_{2}$ ) : le Rhône et la Dranse du Chablais qui représentent à eux deux $85 \%$ des apports d'eau au Léman. Le tableau suivant donne leurs caractêristiques ainsi que celles du Rhin à l'entrée du Bodensee auquel nous les comparons.

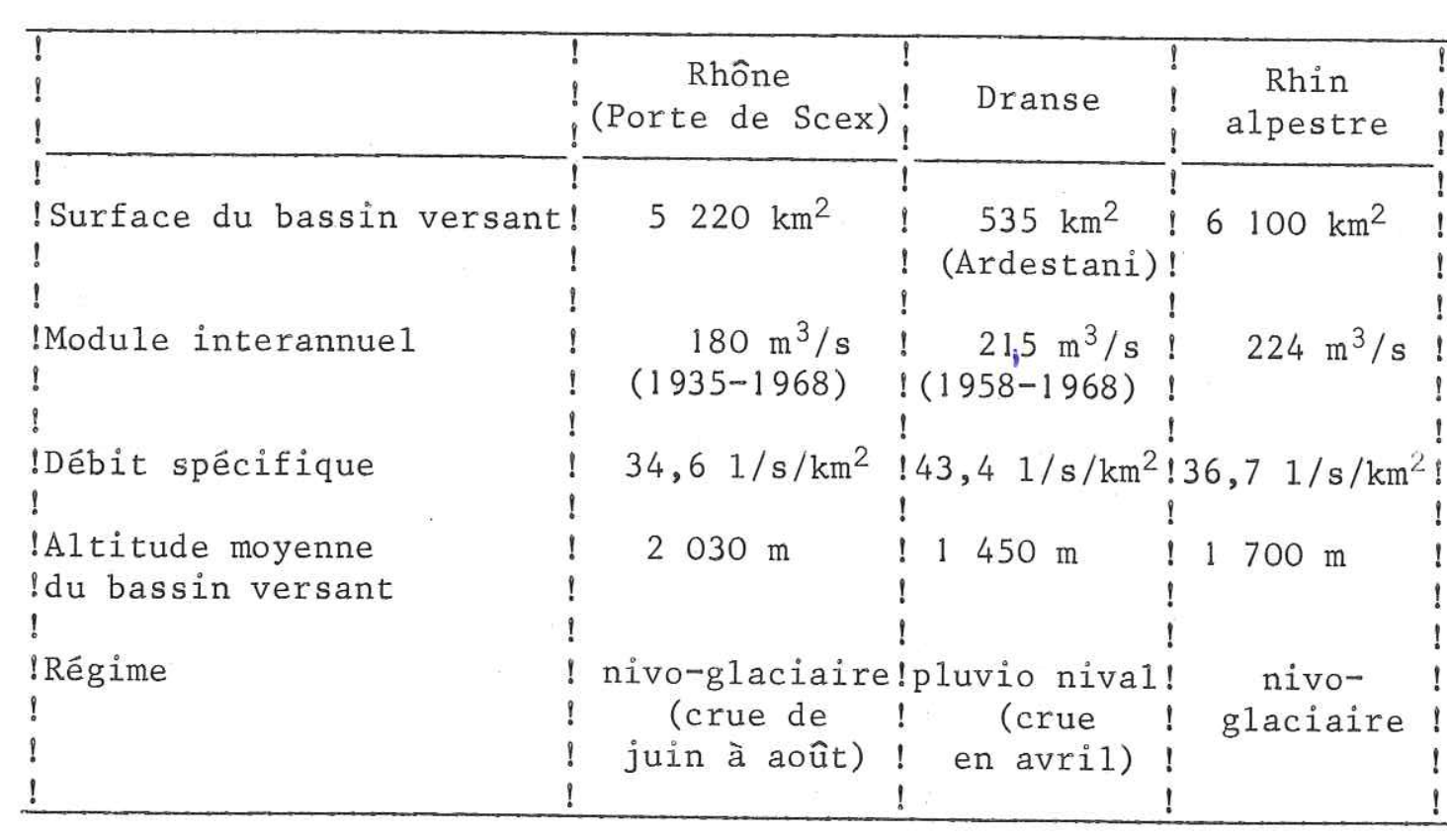

Le comportement de ces deux rivières n'est plus entièrement naturel : 1a Dranse comporte de petits aménagements hydrauliques, le Rhône lui-même n'est pas barré mais de nombreux affluents le sont (barrage de la Grande Dixence, de Moiry, etc...), aussi les débits 
peuvent-i1s être influencés surtout en pêriode de basses eaux. De plus, les points de prélèvements sur la Dranse (pont de Vongy) et sur 1e Rhône (porte du Scex) sont sîtuếs à la fin du parcours de la rivière et donc en aval de différentes sources de pollutions. Néanmoins ainsì que nous le verrons, les effets de la pollution ne sont sensìbles que pour un petît nombre des éléments étudiés. Enfin les bassins versants de la Dranse et du Rhône sont relativement homogènes au poỉnt de vue géographique et géologique, il ne se produira donc pas de phênomènes trop différents au niveau des sousbassîns. Nous pourrons donc quand même étudier le chimisme naturel de ces deux rivières.

Pour 1'établisssement du bìlan des apports au Léman il nous fallaitt des données sur les affluents restants. Nous les avons trouvées dans les rapports internes de la Commission Internationale de Protection des Eaux du Léman et du Rhône, effectués par P. Burkard et quî portent sur les êleêments suîvants : $\mathrm{K}^{+}, \mathrm{Ca}^{++}, \mathrm{Mg}^{++}, \mathrm{Cl}^{-}, \mathrm{NO}_{3}{ }^{-}$. Les concentrations des éléments manquants $\left(\mathrm{SiO}_{2}, \mathrm{Na}^{+}, \mathrm{SO}_{4}{ }^{-}{ }^{-}, \mathrm{HCO}_{3}{ }^{-}\right.$) ont été extrapolées à partîr de la Dranse en raison de la nature identique des bassins versants.

\section{2. - Méthodes d'analyses employées.}

Les analyses furent toutes effectuées au Laboratoire de chimie du Centre de Recherches Géodynamiques, sous la direction de P. BLANC assisté de Mme A. NOIR. Les éléments suivants furent systématíquement dosês : $\mathrm{SìO}_{2}$ dissoute, calcium, magnésium, sodium, potassium, chlorures, sulfates, bicarbonates, carbonates, nitrates par les méthodes suivantes :

- silice : spectrophotométrìe du silico-molybdate réduit par 1'acide aminonaphtol sulfonique. Précision $5 \%$, limite de dosage $0,01 \mathrm{mg} / 1$.
- calcium : complexométrie à 1'EDTA. Prêcision $1 \%$ imit.e de dosage $0,01 \mathrm{mg} / 1$.

- magnésium : complexométrie à I'EDTA (différence entre duretể totale et calcium) ; depuis 1969 par Absorption Atomique. Prêcision $5 \%$ par complexométrie et 1 \% par Absorption Atomique, limite de dosage $0,005 \mathrm{mg} / 1$.

- sodium et potassium : photométrie de flamme en êmìssion, depuis 1969 par Absorption Atomique. Précision $5 \%$ par photométrie et $2 \%$ par Absorption Atomique, limite de dosage $0,01 \mathrm{mg} / 1$.

- chlorures: spectrophotométrie au thiocyanate mercurique en présence de fer ferrique, en milieu perchlorique. Précision $2 \%$, limite de dosage $0,05 \mathrm{mg} / 1$.

sulfates : turbidimétrie. Précision $5 \%$, limite de dosage $2 \mathrm{mg} / 1$.

- bicarbonates : titrimétrie à l'acide sulfuríque /50 en présence d'indicateur mixte. Précision $2 \%$, limite de dosage $0,1 \mathrm{meq} / 1$

- carbonates : titrimétrie à l'acíde sulfurique N/50 en présence de phénolphtaléine. Précision $10 \%$, limite de dosage $0,15 \mathrm{meq} / 1$.

- nitrates : spectrophotométrie à l'acide phénol disulfonique. Précision $5 \%$, limite de dosage $0,05 \mathrm{mg} / 1$. 


\section{3. - Variation des éléments dissous dans le Rhône.}

\subsection{1. - Caractères du bassin.}

Le bassin du Rhône est presque entièrement situé dans le Valais, les terraîns traversés sont à la fois crìstallìns (Gotthard, Aar, Mt Blanc) et calcaîres (Préalpes du Chablais et du Valais). Les eaux sont bicarbonatées calciques rìches en sulfates et magnésium. Depuis Forel (1895) et Delebecque (1898), les travaux sont peu nombreux. La présente étude porte sur 25 analyses réparties de Novembre 1967 à Mars 1970, les résultats complets sont reportés sur le tableau $\mathrm{II}_{2}$ en annexe de ce chapitre.

\subsection{2. - Corrélation entre la concentration et le débit.}

La grande variabilité de la concentration avait déjà été remarquée par Delebecque (1898) et Forel (1895), ce dernier avait déjà écrit : "pour 1e Rhône comme pour 1a Dranse la teneur en matière dissoutes est fonction inverse du débit" (Le Léman tome II, page 597) Examinons les relations entre la concentration $C_{d}$ et le débit instantané $Q_{1}$. Elles sont du type

$$
C_{d}=A Q_{1}^{B}
$$

$A$ et $B$ sont des coefficients dépendant des unités choisies.

Le graphe de cette fonction est une droite en coordonnées bi logarithmique. Pour chaque élément nous allons voir la qualité de la corrélation et la dispersion des mesures autour de cette droite expérimentale. Nous avons pris le débit instantané et non pas journalier : en effet de grosses variations de débit peuvent être quelquefois enregistrées sur le Rhône Alpestre et encore plus souvent sur la Dranse du Chablais en 1 'espace de quelques heures comme le montre 1a figure $\mathrm{II}_{6}$.
- bicarbonates: (figure $\mathrm{II}_{\mathrm{I}_{\mathrm{a}}}$ ). Corrêlation moyenne, faible variation, dispersion de $70 \mathrm{mg} / 1$ à $120 \mathrm{mg} / 1$.

- calcium: (figure $\mathrm{II}_{\mathrm{l}_{\mathrm{b}}}$ ). Corrélation nette, dispersion de $32 \mathrm{mg} / 1$ à $65 \mathrm{mg} / 1$

sulfates : (fígure $I I_{1_{c}}$ ). Corrélation nette avec une variation du simple au trîple, de $27 \mathrm{mg} / 1$ à $91 \mathrm{mg} / 1$.

- magnésium: (figure $\mathrm{II}_{\mathrm{I}_{d}}$ ). Bonne corrélation, variation du simple au triple, de $2,6 \mathrm{mg} / 1$ à $8,8 \mathrm{mg} / 1$.

- sodium : (figure $\mathrm{II}_{1} \mathrm{e}$ ). Corrélation moyenne, variations très fortes, de $1,6 \mathrm{mg} / 1$ à $6,6 \mathrm{mg} / 1$.

- potassium: (figure $\mathrm{II}_{1_{\mathrm{f}}}$ ). Corrélation médiocre, variations de $1 \mathrm{mg} / 1$ à $2,3 \mathrm{mg} / 1$.

- silice : (figure $\mathrm{II}_{\mathrm{I}_{\mathrm{g}}}$ ). Corrélation médiocre, variations de $2 \mathrm{mg} / 1$ à $4,5 \mathrm{mg} / 1$. Cette variation de la silice est intéressante, en effet, certains auteurs comme Davis (1964) ont signalé la constance de la silice vis-à-vis des débits. Nous discuterons du comportement plus loin $(2.6 .2$ 。)

- chlorures : (figure $\mathrm{II}_{\mathrm{l}_{\mathrm{h}}}$ ). La corrélation est assez bonne. Davis I'avait déjà remarqué en partîculier pour les rivières Matanuska et Connecticut. Ceci est remarquable car les chlorures son souvent rejetés par les usines, les concentrations sont alors beaucou plus indépendantes du débit. Par exemple le 8 janvier 1968 nous avons dosé $16,6 \mathrm{mg} / 1$ de $\mathrm{Cl}^{-}$pour un débit de $70 \mathrm{~m}^{3} / \mathrm{s}$, le point représentati de ce prélèvement n'a pas été porté sur la figure. Cette corrélation chlorures-débits pourrait signifier que le Rhône n'est pas encore trè atteint par la pollution industrielle.

- nitrates: (figure $\mathrm{II}_{1_{1}}$ ). La corrélation avec les débits est faible et deux points ( 6 et 7 Août 1968) sortent du nuage "norma1". Les teneurs en nitrates sont donc très variables ce qui est 

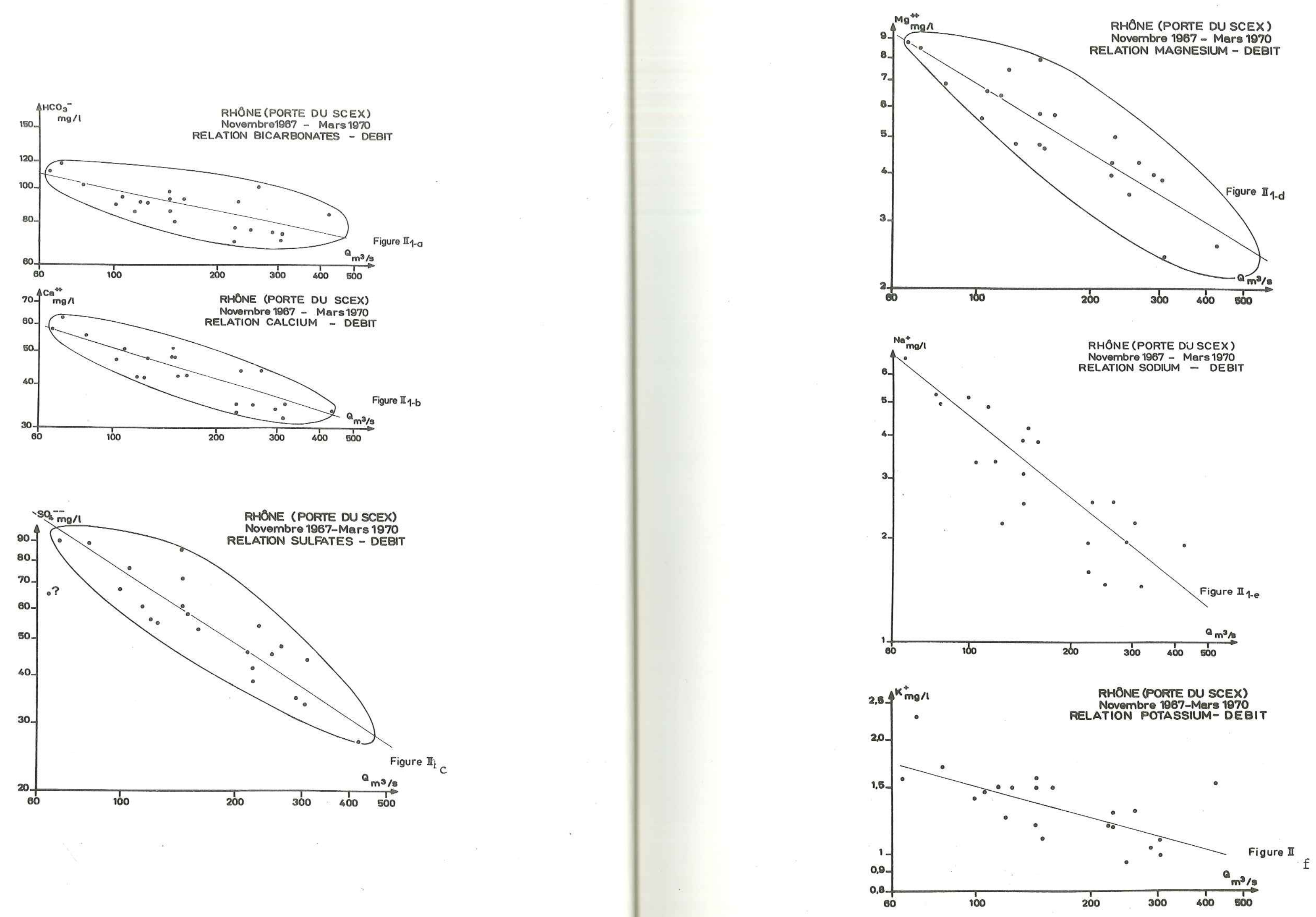


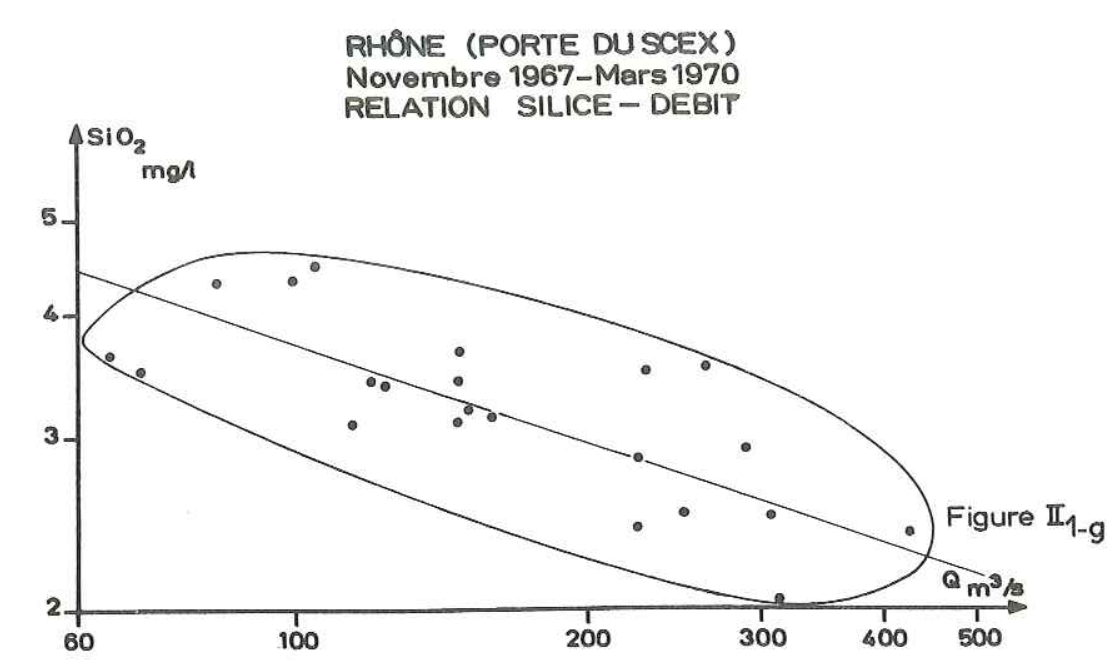

Il ne faut pas s'étonner de la grande dispersion des concentrations en nitrates. Des études poussées ont été réalisées par 1'"Internationale Gewässerschutz Kommisssion für den Bodensee" qui remarque, dans son rapport $n^{\circ} 6$ sur les apports au Bodensee en éléments polluants, des variations très fortes en quelques heures sans que le débit du Rhin ait sensiblement varié :

\begin{tabular}{|c|c|c|c|}
\hline Mois & Eléments & $\begin{array}{l}\text { Variation sur } \\
? \text { jours (mg/1) } \\
\end{array}$ & $\begin{array}{l}\text { Variation sur } \\
1 \text { jour (mg/1) }\end{array}$ \\
\hline !Eévrier 1961 & !Chlorures & $1,05-1,55$ & $1,05-1,55$ \\
\hline :Débit de & !Phosphore total & $0,06-0,17$ & $0,06-0,17$ \\
\hline $101 \mathrm{~m}^{3} / \mathrm{s}$ à $133 \mathrm{~m}^{3} / \mathrm{s}$ & Azote total & $0,95-2,56$ & $1,07-2,56$ \\
\hline Juillet 1961 & !Chlorures & $0,3-2,4$ & $0,3-2,0$ \\
\hline :Débit variable de & !Phosphore total & $0,05-0,45 !$ & $0,05-0,30$ \\
\hline $300 \mathrm{~m}^{3} / \mathrm{s}$ à $545 \mathrm{~m}^{3} / \mathrm{s}$ & Azote total & $1,06-2,44$ & $1,06-2,32$ \\
\hline !octobre 1961 & !Chlorures & $0,7-5,4$ & $0,7-4,2$ \\
\hline !Débit de & !Phosphore total! & $0,01-0,28 !$ & $0,01-0,28$ \\
\hline $84 \mathrm{~m}^{3} / \mathrm{s}$ à $126 \mathrm{~m}^{3} / \mathrm{s}$ & Azote total & $\begin{array}{l}0,8 \\
!\end{array}$ & $0,8-1,96 !$ \\
\hline
\end{tabular}

Aucune variation caractéristique des saisons n'a pu être mise en évidence dans aucun des éléments étudiés : à débit égal les concentrations d'hiver et d'été ne montrent pas de différences significatives. Il est possible que ces liaisons existent pour les bicarbonates ou la silice mais nous ne disposions pas d'assez d'ana1yses.

\subsection{3. - Corrélation entre les éléments.}

Nous avons effectué plusieurs diagrammes de dispersion ("scatter diagram") pour déterminer les relations entre les éléments. 

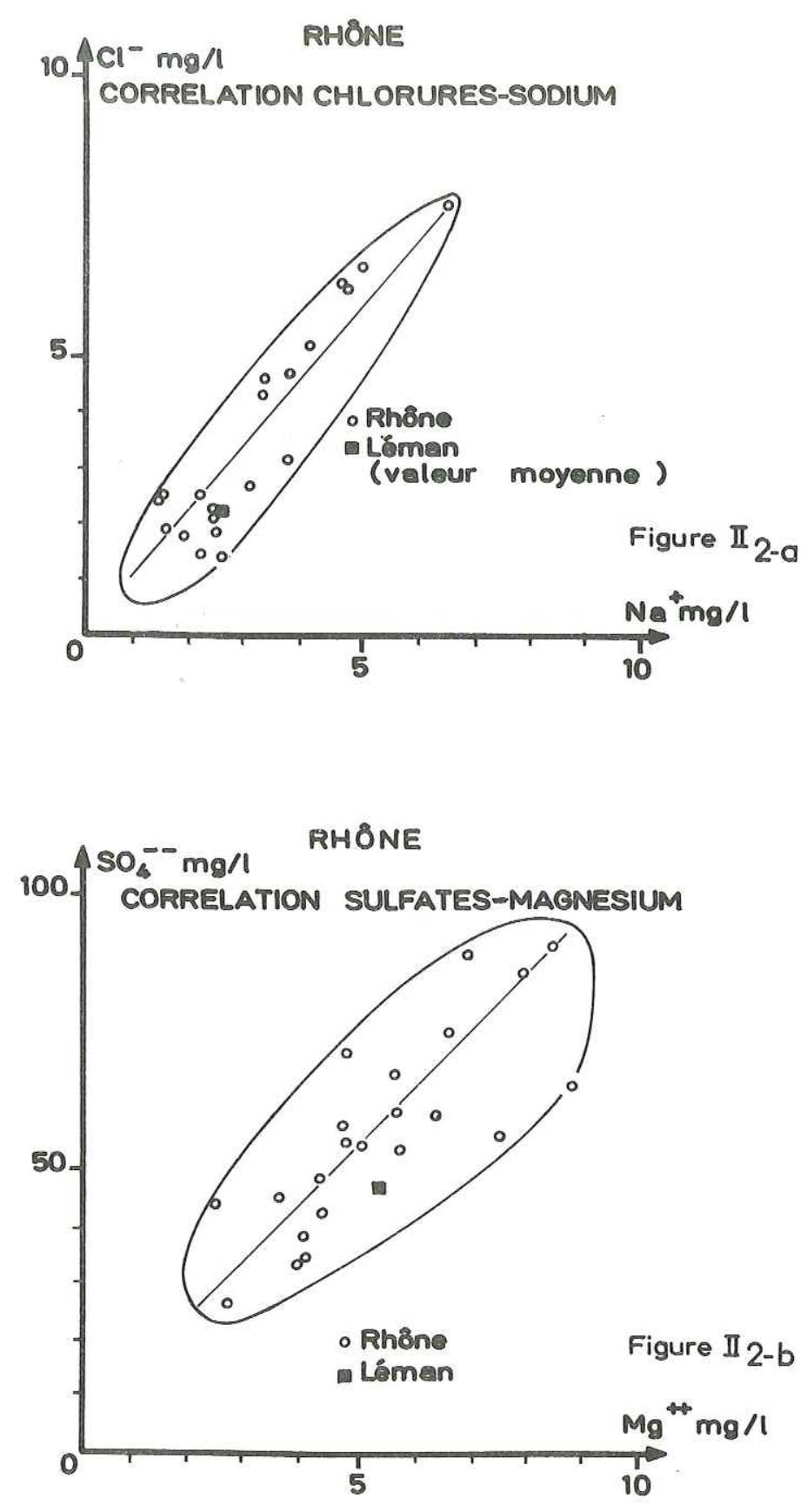
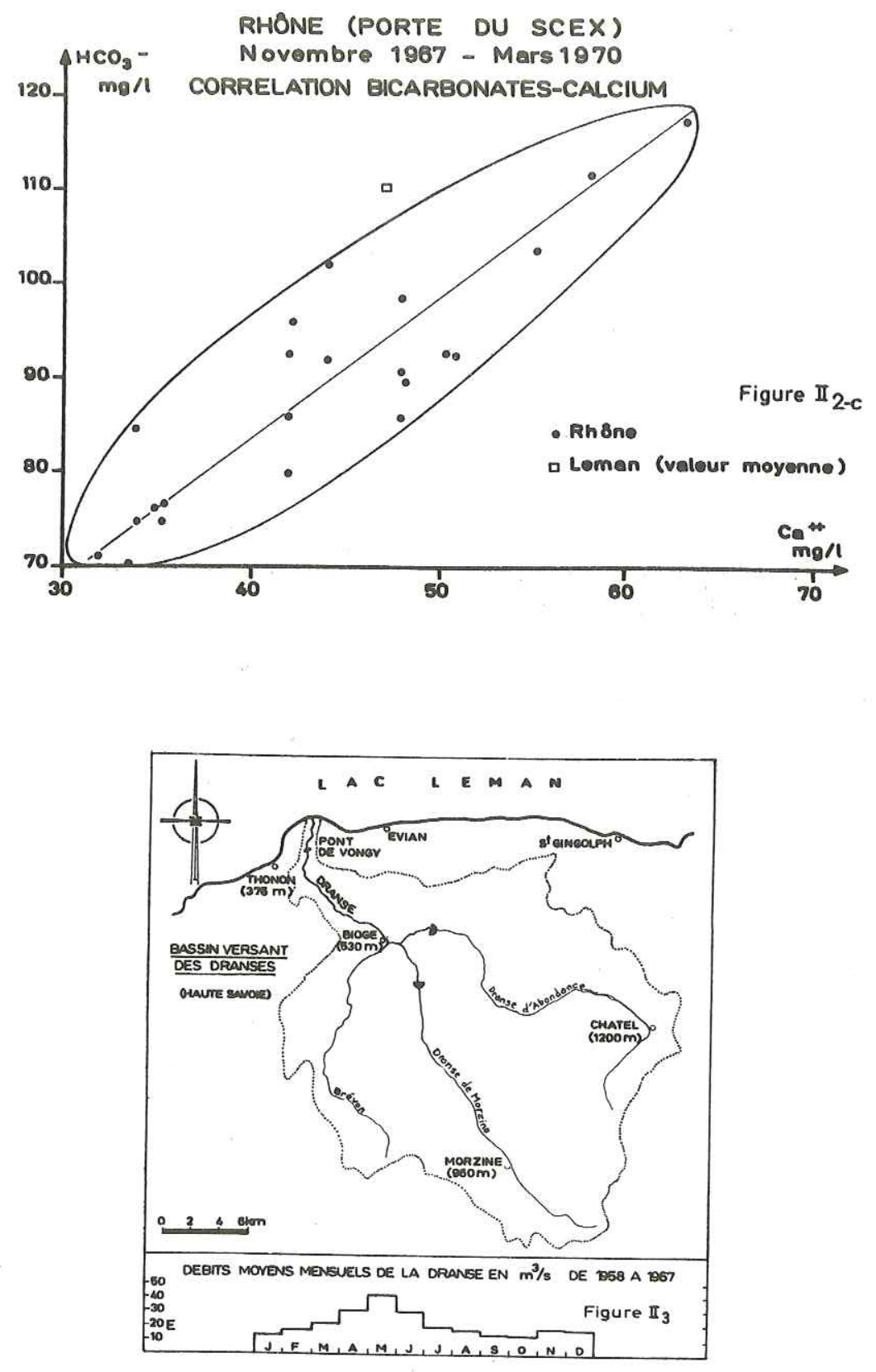
La corrélation la plus forte s'observe entre les chlorures et le sodium (figure $\mathrm{II}_{2_{a}}$ ) on note également une corrélation nette entre les sulfates et le magnésium (fìgure $\mathrm{II}_{2 \mathrm{~b}}$ ), et les bicarbonates et le calcium (fígure $\mathrm{II}_{2_{c}}$ ). Ces corrélations ne sont pas étonnantes, elies correspondent à des origines géochìmiques communes et aux lỉaisons chimîques entre les éléments.

\section{4. - Variations des éléments dissous dans la Dranse.}

2.4.1. - La Dranse du Chablaís est formée par 1a rêunion de trois rivières : Ia Dranse d'Abondance, la Dranse de Morzine et la Dranse de Bellevaux ou Brevon (figure $\mathrm{II}_{3}$ ). L'ensemble du bassin est situé dans les Préalpes du Chablais, nappes calcaires et grêseuses recouvertes quelquefois par des dépôts quaternaires fluvioglaciaires. Les principales études du chimisme de la Dranse ont été réalisées par Ardestani (1965), Sayar (1966) et Blanc (1968). Comme 1e Rhône, la Dranse est bicarbonatêe calcique, sa teneur en sulfate est légèrement plus forte. De Novembre 1967 à Décembre 1969 une cinquantaine d'analyses totales ont été réalisées. Les prélèvements ont été répartis également sur toute l'année et des études particulières ont été réalisées sur certains épisodes hydrologiques (crue d'orage, crue de fonte de neige, crue et étiage exceptionnels). Enfin nous avons tenté de différencier les eaux des trois affluents composant la Dranse du Chablais.

\subsection{2. - Corrélation entre la concentration et le débit.}

Comme pour le Rhône la variation principale des éléments dissous est due au débit.

- bicarbonates: (figure $\mathrm{II}_{4_{a}}$ ). La corrélation est assez bonne, une certaine dispersion peut s'observer. En effet les valeurs sont légèrement plus fortes en hiver qu'en été, cette disdistinction saisonnière est sans doute reliée ici à la température. La variation observêe est du simple au double (120 mg/1 à $250 \mathrm{mg} / 1$ ).
- sulfates: (figure $\mathrm{II}_{4}$ ). La corrélation est assez bonne : on observe une dispersion dans les valeurs, surtout pour les débits extrèmes. Nous 1 'expliquerons par la suite comme pour le Rhône. Le domaine de variation est très large (12 mg/1 à $160 \mathrm{mg} / 1$ ).

- calcium: (fîgure $\mathrm{II}_{4}$ ). La corrélation est bonne, 1a variation est du simple au double ( $52 \mathrm{mg} / 1$ à $110 \mathrm{mg} / 1)$.

- magnésium : (figure $\mathrm{II}_{4 \mathrm{~d}}$ ). La corrêlation est bonne, les teneurs varient du simple au quadruple $(4,3 \mathrm{mg} / 1$ à $16 \mathrm{mg} / 1)$.

- sodium : (figure $\mathrm{II}_{4} \mathrm{e}$ ). Le sodium marque une très grande dispersion, la corrélation avec le débit est très faible, le même phénomène s'observe pour le potassium. Il faut sans doute voir là un indice de pollution : en effet, si l'ensemble des valeurs est groupé entre 0,7 et $3,5 \mathrm{mg} / 1$ pour 1 e sodium, on note une valeur de $9,3 \mathrm{mg} / 1$ à l'étiage très prononcé de Novembre 1969. De même pour 1e potassium les valeurs sont toutes comprises entre $0,5 \mathrm{mg} / 1$ et $2 \mathrm{mg} / 1$ sauf une à $3,3 \mathrm{mg} / 1$.

- silice: (figure $\mathrm{II}_{\mathrm{f}_{f}}$ ). La dispersion des teneurs est très forte, et aucune corrélation avec le débit ne peut être mise en évidence. Nous avons là un exemple typique de la non-variation de la silice avec le débit. Ce phénomène a déjà été remarqué pour certaines rivières (Davis, 1964), mais pour d'autres (Carbonnel, 1965), la silice varie énormément avec le débit.

Par contre nous avons observé un groupement net pour chaque crue : 1a teneur est relativement constante quelque soit le débit. I semblerait donc que la silice provienne beaucoup plus des eaux superficielles (ruissellement et imprégnation des sols) que des eaux souterraines.

- chlorures : (figure $\mathrm{II}_{4} \mathrm{~g}$ ). Cet élément est très dispersé, les valeurs observées vont de 0,2 à $4 \mathrm{mg} / \mathrm{l}$. Contrairement au Rhône, nous avons donc là un indice net de pollution. 

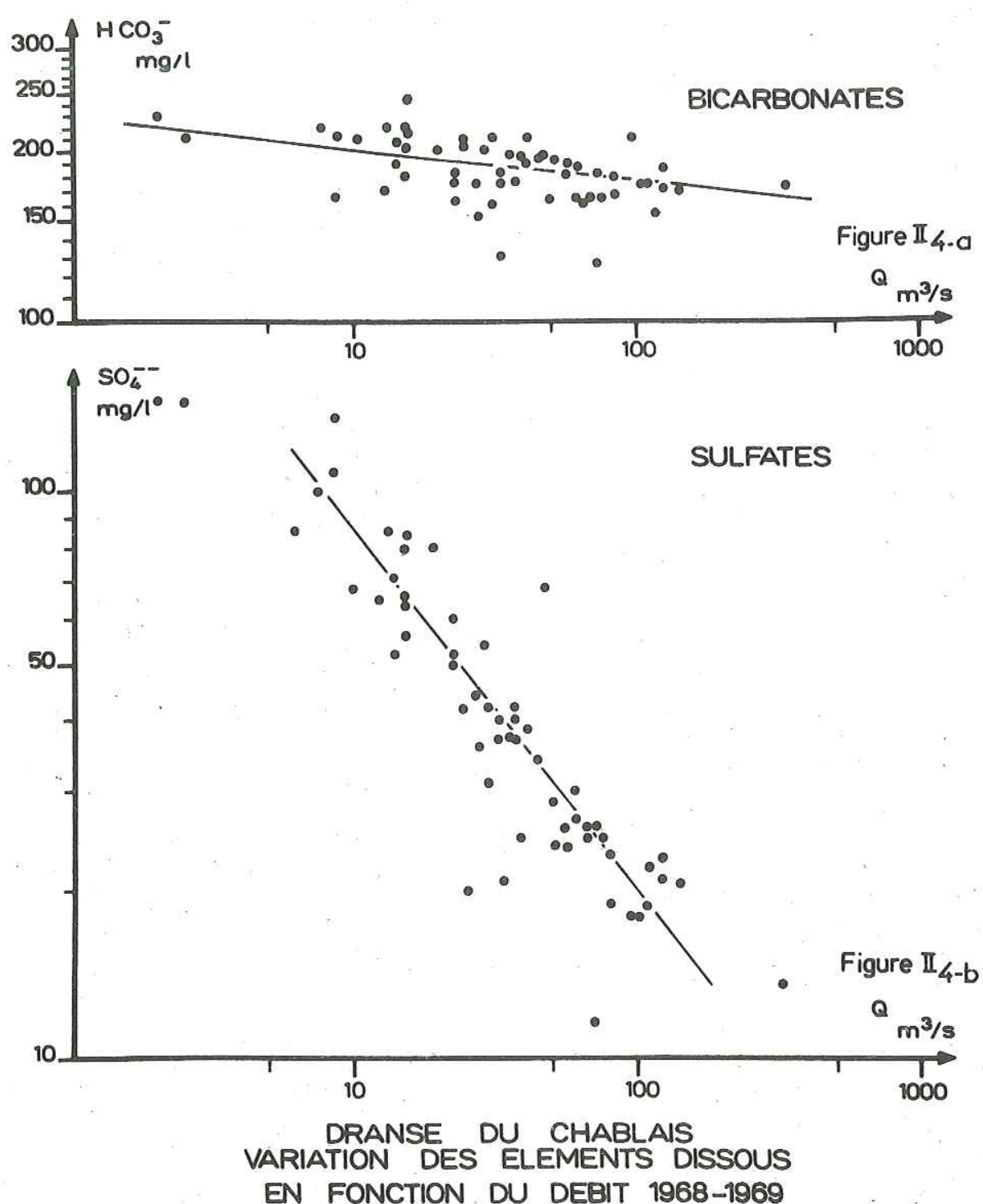

EN FONCTION DU DERIT
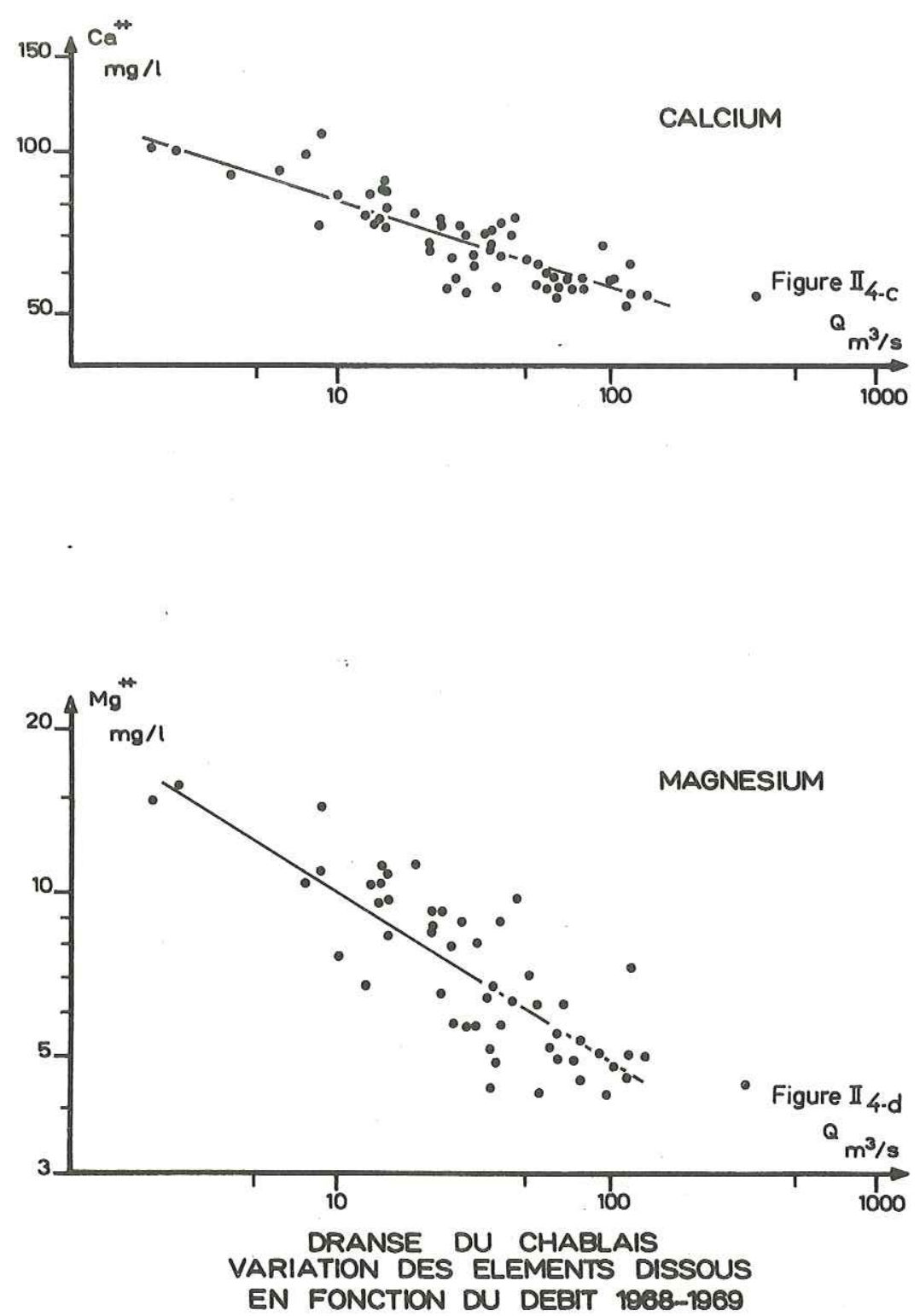

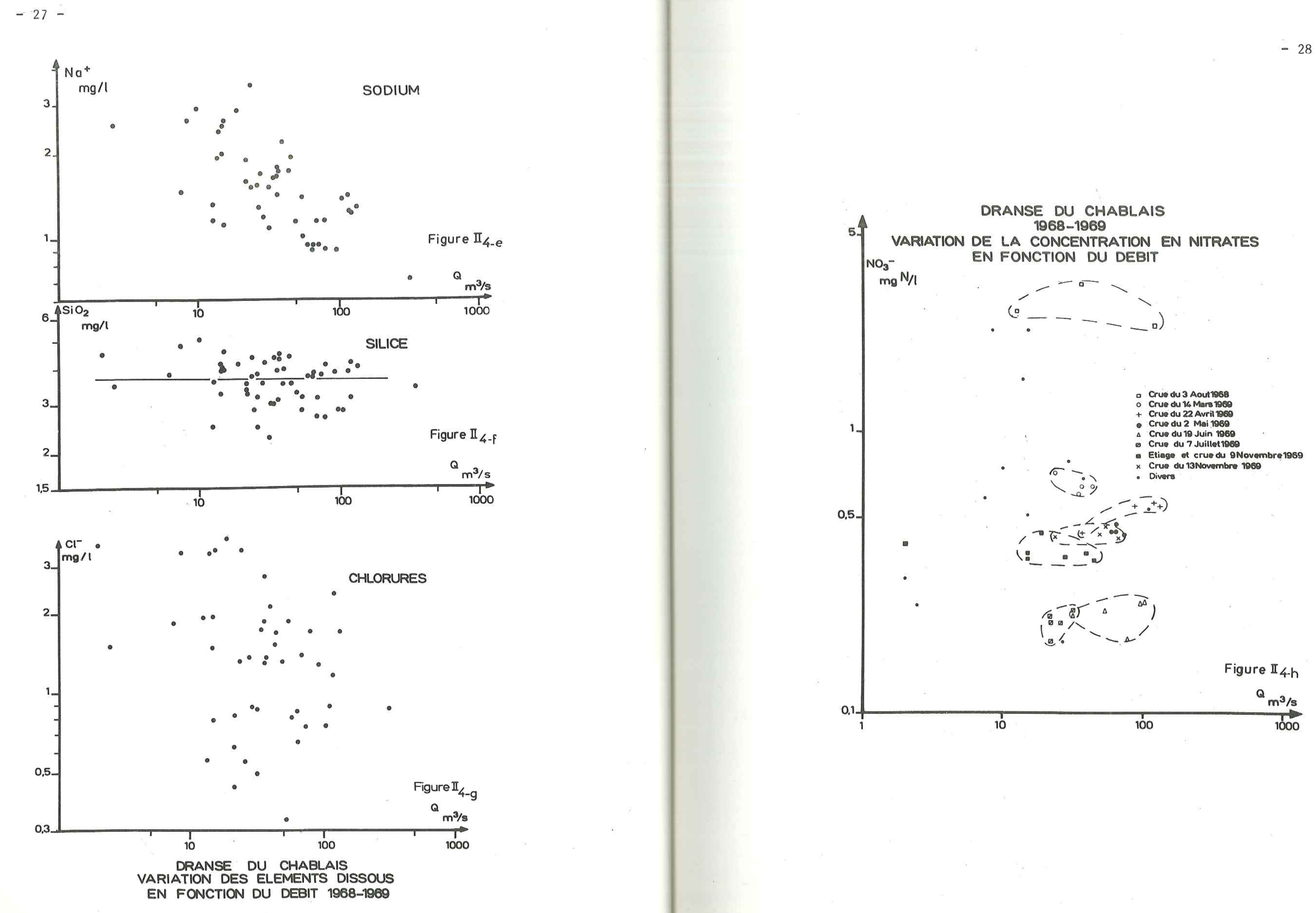
- nitrates: (figure $\mathrm{II}_{4} \mathrm{~h}$ ). Ils sont très dispersés entre $0,18 \mathrm{mg} / 1$ et $3,5 \mathrm{mg} / 1$ d'azote équivalent. Une étude plus précise montre un groupement très net des valeurs par crue élémentaire. Ceci peut s'expliquer si on considère que la source principale des nitrates est constituée par des engrais agrìcoles, la teneur observée sera donc fonction de la période d"épandage et du ruissellement.

\subsection{3. - Corrélation entre les éléments}

Les diagrammes de dispersion réalìsés ont montré les mêmes corrélations que pour le Rhône. La corrélation entre le sodium et les chlorures (figure $\mathrm{II}_{5_{a}}$ ) est satisfaisante si on élimine le prélèvement du 7 Novembre 1969 très poliué. La corrélation entre le Ca et les bicarbonates (figure $\mathrm{II}_{5_{\mathrm{b}}}$ ) est plus complexe : en effet, 1 'ensemble des valeurs est assez dispersé mais on peut observer une très bonne corrélation à l'échelle de la crue. Ce phénomène est encore plus visible pour la corrélation magnésium-sulfates (figure $\mathrm{II}_{5_{c}}$ ). Nous avons reporté sur ces deux dernières figures les valeurs des crues du 21 Septembre 1968, du 9 Novembre 1969 et du 13 Novembre 1969. On peut expliquer cette particularité de deux façons :

- chaque crue élémentaíre correspond à une certaine proportion d'eau des trois Dranses dont le comportement chimique n'est pas rigoureusement identique. Cette explication serait surtout valable pour la corrélation $\mathrm{Mg}-\mathrm{SO}_{4}$

- chaque crue intervient dans un contexte climatique et hydrologique déterminé (température, degré de lessivage des sols, etc...), cette explication s'appliquerait surtout à la corrêlation $\mathrm{Ca}-\mathrm{HCO}_{3}$.

Nous avons également représenté le diagramme de dispersion entre la silice et les nitrates. Les valeurs de nitrates supérieures à $1 \mathrm{mg} / 1 \mathrm{n}$ 'ont pas été prises en considération (figure $\mathrm{II}_{5_{\mathrm{d}}}$ ) : une corrélation positive est nette et on peut remarquer que -mise à part la crue du 21 Septembre 1968- les valeurs se groupent bien suivant la saison. Les valeurs les plus élevées du couple Silice- $-\mathrm{NO}_{3}{ }^{-}$se rapportent
DRANSE DU CHABLAIS

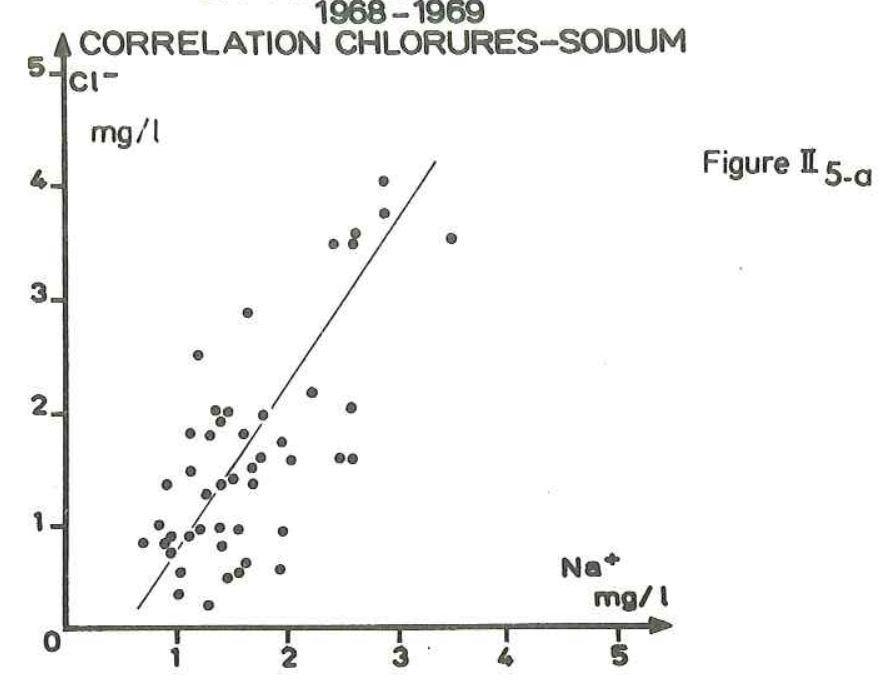

DRANSE DU CHABLAIS CORRELATION BICARBONATES=CALCIUM

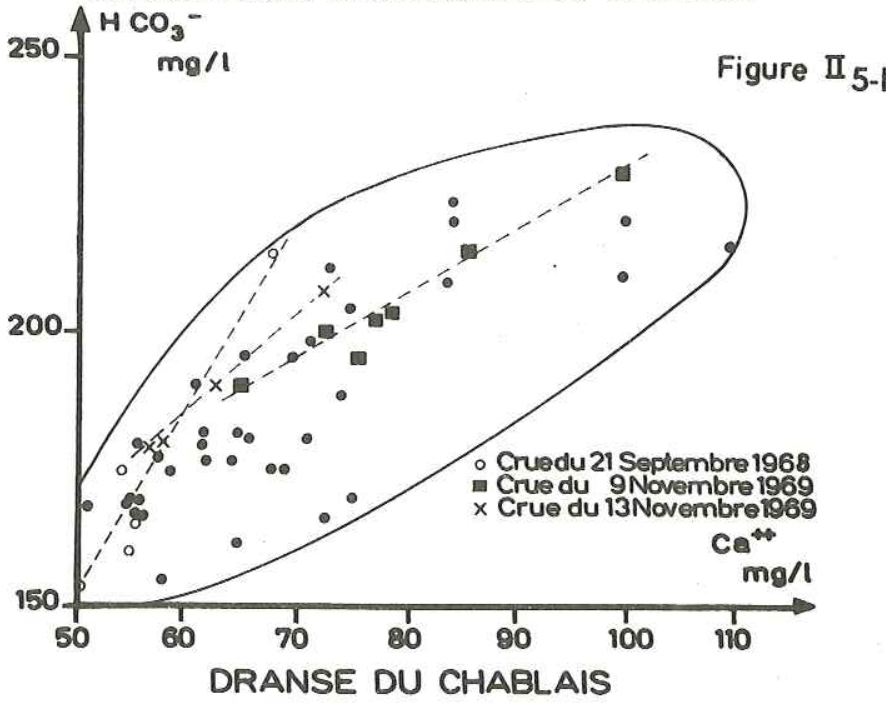




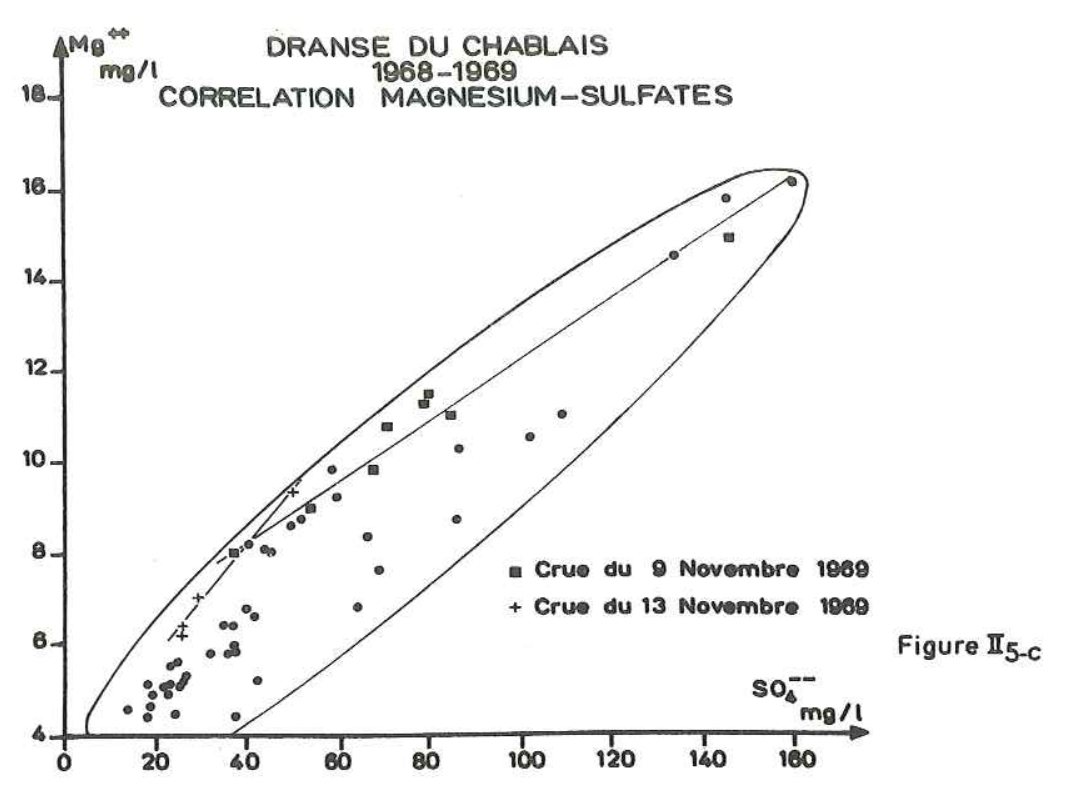

à I'hiver, les plus faỉbles à l'été. Cette relation n'est pas tellement surprenante si on consìdère que l'origine principale des deux éléments est sans doute la même : eaux de ruissellement et eaux d'imprégnation.

\subsection{4. - Etude du chimisme de certains épisodes hydrologiques.}

Nous avons pu étudier un certain nombre de crues, ce terme étant ici compris comme une augmentation brusque de débit sans tenir compte de la valeur atteînte par le maximum. Les corrélations entre la concentration des éléments et le débit ne sont pas la plupart du temps meilleures que les corrélations générales. Nous avons déjà vu que certaines données sont caractérístîques de chaque crue : les nitrates et $1 \mathrm{a}$ silice qui varient peu et les proportions $\mathrm{Ca}-\mathrm{HCO}_{3}$ et $\mathrm{Mg}-\mathrm{SO}_{4}$. Deux épisodes extrêmes ont pu être étudiés.

La crue du 21 Septembre 1968: 1e débit a atteint au moins $400 \mathrm{~m}^{3} / \mathrm{s}$ soit trois fois plus que les crues annuelles, la durée de retour d'une telle crue a été estimée à 10 ans (Hubert et al, 1969). Un prêlèvement proche du maximum de crue $\left(310 \mathrm{~m}^{3} / \mathrm{s}\right)$ a pu être analysé, les valeurs de sulfates descendent jusqu'à $14,2 \mathrm{mg} / 1$, ce qui est le minimum observê sur plus de deux ans. Notons que le point représentatif de cette crue est systématiquement à l'écart de la variation générale : nous avons là un changement d'échelle physique, les mêmes corrélations concentrations-débits quî sont valables pour des variations de débits normals $\left(5 \mathrm{~m}^{3} / \mathrm{s}-150 \mathrm{~m}^{3} / \mathrm{s}\right)$ ne s'appliquent plus aux crues exceptionnelles : le comportement chimique habituel de 1a rivière a changé. En effet, le coefficient de ruissellement ca1culé par les méthodes classiques atteint $90 \%$ au maximum de crue, on pourrait s'attendre à des compositions chimiques proportionnelles à $10 \%$ d'eau d'avant la crue et $90 \%$ d'eau de pluie, il n'en est rien et les teneurs observêes sont nettement plus fortes. Plusieurs explications sont possibles : une partie des eaux de ruissellement met en charge les eaux d'imprégnation des sols plus chargées en ions dissous, 


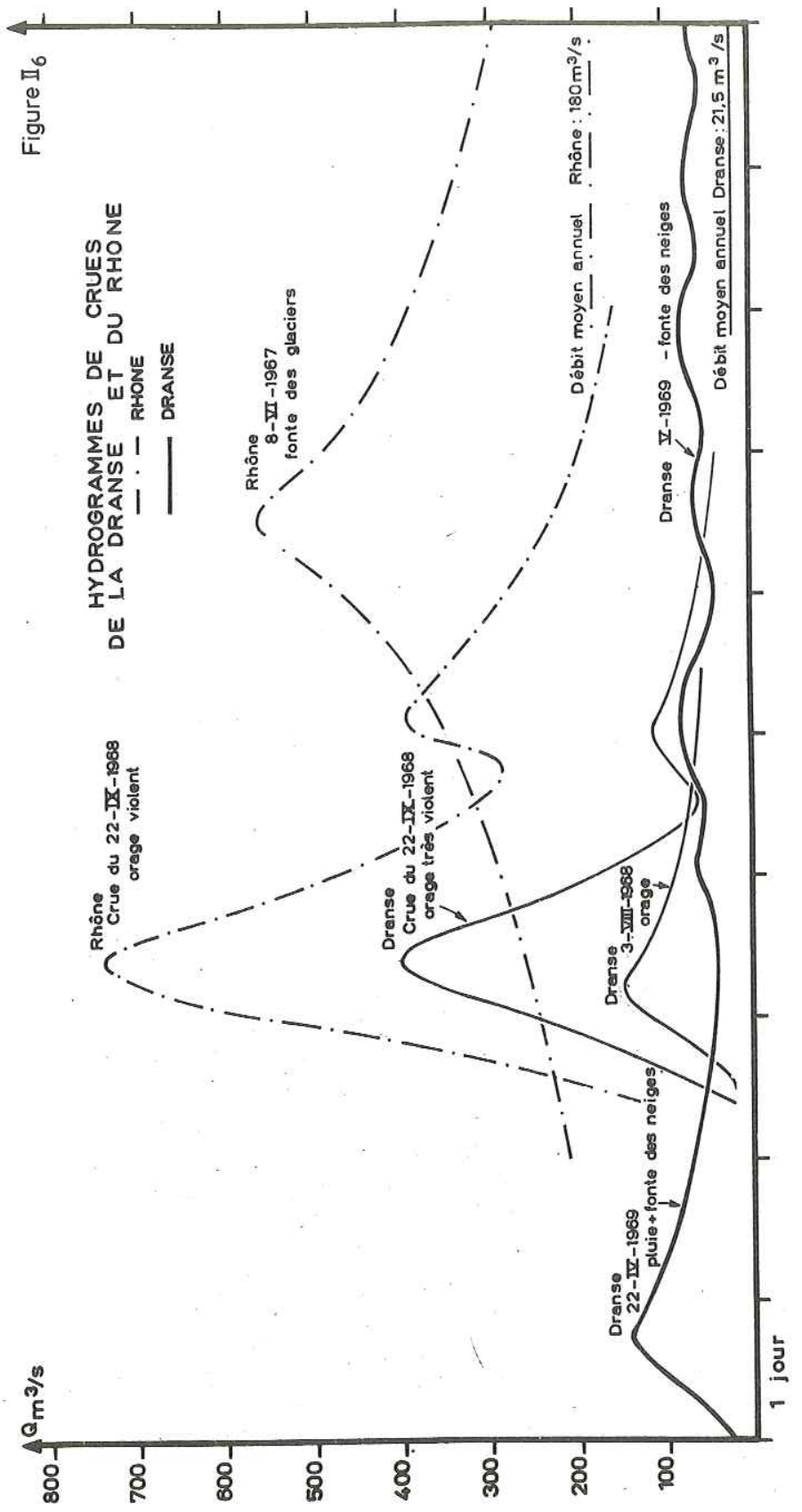

ou bien il y a mise en solution partielle des matières en suspensions dans les eaux. Il est également possîble que les coefficients de ruisseliement soîent surestìmés par les méthodes classìques et que la part des eaux souterraines -plus chargées- dans les crues soit. supérieure (Crouzet et al, 1970).

\section{L'étiage exceptionnel d'Octobre 1969 : à la suite d'une} sécheresse très prolongée dont le temps de retour sur l'ensemble des Alpes a été estìmé à 100 ans (Saîntignon, 1969), le débît de la Dranse est descendu à $2 \mathrm{~m}^{3} / \mathrm{s}$. Les analyses effectuées se situent toutes en dehors de la variation générale et sont inférieures à celles qu'on attendrait. L'enrichissement chimique des eaux est donc limité, les teneurs suivantes correspondent donc aux teneurs des eaux souterraines -en mg/1- qui constituent alors la totalité des eaux de 1a Dranse.

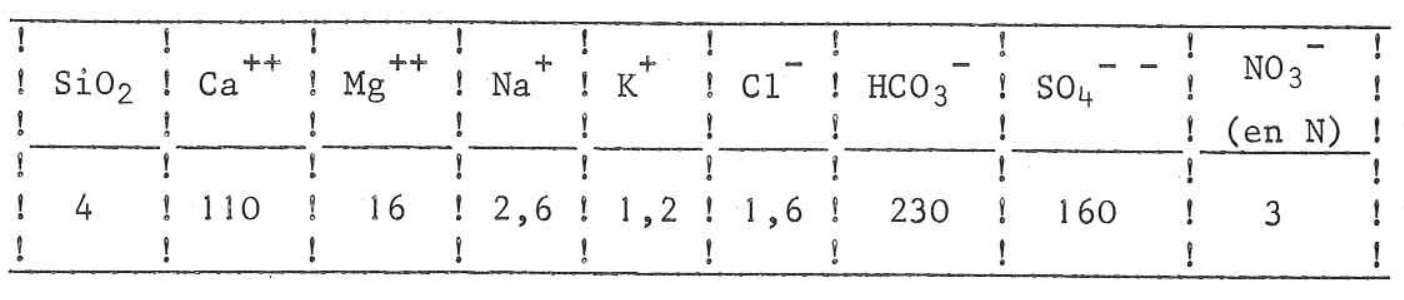

Notons que les éléments $\mathrm{K}^{+}, \mathrm{Na}^{+}, \mathrm{NO}_{3}{ }^{-}, \mathrm{Cl}^{-}$et $\mathrm{SiO}_{2}$ n'ont pas un enrichissement très élevê, comme nous $1^{\prime}$ avons dêjà dit leur origine est plus dans les eaux de lessivages des sols que dans les eaux souterraines.

Enfin une étude particulière de la crue annuelle de fonte des neiges a été réalisée en 1969 (figure $\mathrm{II}_{6}$ ). L'hydrogramme est typique et des prêlèvements ont été faits lors de la montée des eaux le 25 Avril et pendant les oscillations journalières de fontes des neiges (2 Mai) de $60 \mathrm{~m}^{3} / \mathrm{s}$ à $75 \mathrm{~m}^{3} / \mathrm{s}$. Pendant ces oscillations, les teneurs des éléments principaux (ions $\mathrm{Ca}^{++}, \mathrm{Mg}^{++}, \mathrm{HCO}_{3}{ }^{-}$et $\mathrm{SO}_{4}{ }^{-}$) 
sont tout à fait "normales" compte tenu des dêbits. Par contre pour les autres élêments, les valeurs sont très particulières : silice très forte $(3,9 \mathrm{mg} / 1)$, mais chlorures $(0,65$ à $0,85 \mathrm{mg} / 1)$, nitrates $(0,45 \mathrm{mgN} / 1)$, sodium $(0,95 \mathrm{mg} / 1)$ et potassium $(0,6-0,85 \mathrm{mg} / 1)$ atteígnent là leurs valeurs les plus basses de toute $1^{\text {'année. }}$ du Chablais.

\subsection{5. - Oxigine géographique des éléments de la Dranse}

La Dranse du Chablaîs est formée de la réunion à Bioge de trois rivières assez comparables tant par leur dêbit spécifique, leur régime que par la nature de leur bassin versant (figure $\mathrm{II}_{3}$ ). Nous avons trouvé dans les deux travaux sur la Dranse de Morzine (Sayar, 1966) et d'Abondance (Ardestani, 1965), des résultats d'analyses chimiques que nous avons comparês aux nôtres (tableau $\operatorname{II}_{1}$ en annexe). II en ressort que :

- les trois rivières ont une composition générale analogue : eaux bicarbonatées, sulfatées calciques.

- les Dranses d'Abondance et de Morzine sont très semblables, les différences entre les concentrations n'excédant pas $20 \%$ pour des épisodes hydrologiques semblables. La Dranse de Morzine semble néanmoins toujours légèrement moins minéraliseée que celle d'Abondance.

- la Dranse de Bellevaux ou Brevon se distingue nettement par sa plus forte minéralisation en calcium et surtout en magnésium et

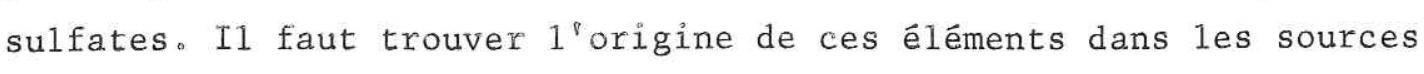
du cours inférieur du Brevon qui traversent les dolomies et gypses triasiques et dont les teneurs peuvent atteindre $1000 \mathrm{mg} / 1$ en $\mathrm{SO}_{4}{ }^{-}$-, $450 \mathrm{mg} / 1 \mathrm{en} \mathrm{Ca}^{++}$et $22 \mathrm{mg} / 1$ en $\mathrm{Mg}^{++}$.

\subsection{6. - Origine géochimique des éléments dissous de la Dranse.}

Nous avons vu que les éléments se groupaient nettement en trois, suivant leur variation, en fonction des débits, des crues, de la fonte des neiges, etc...

Les íons calcìum, magnếsîum, bìcarbonates et sulfates sont amenés princípalement par les eaux souterraìnes chargées; lors des crues, il y a dilution de celles-cì dans des eaux de ruissellement beaucoup moins minéralisées. Ce phénomène s'observe le mìeux pour les sulfates dont la variation en fonction du débit est de 1 à 10 .

La silice provient sans doute des eaux superficielles, princípalement des eaux d'ìmprégnation des sols. Sa teneur ne dépend pas du tout du débît maîs plutôt de 1 'époque de 1 'année et de la crue.

Les nitrates et aussi les chlorures, sodium et potassium, proviennent essentiellement du ruissellement des eaux sur les terrains cultivés. Chaque crue a des teneurs bien particulières dêpendant des crues antérieures, des engraís répandus etc... Ainsi pour la crue de fonte des neiges, ces quatres éléments sont en très faibles quantités dans 1a Dranse. La part des apports artificiels est presque nulle et les teneurs sont alors très proches de celles des pluies, notamment pour les chlorures, ce quỉ signifie que ces éléments ne proviennent qu'en faibles proportions de la dissolution des roches.

\section{5. - Bilan des apports au Léman.}

\subsection{1. - Mode de calcul des bilans.}

Nous avons vu la grande variabilité des compositions chimiques du Rhône et de la Dranse suivant le débit, parfoís la saison, le taux de pollution, la nature de l'épisode hydrologique, etc... Les éléments majeurs sont essentiellement liés au débit, il en résulte que 1a somme des éléments dissous le sera également. Nous avons porté sur les figures $\mathrm{II}_{7}$ et $\mathrm{II}_{8}$ la variation en fonction du débit instantané de la somme des élếments dissous : $\mathrm{SiO}_{2}, \mathrm{Ca}^{++}, \mathrm{Mg}^{++}, \mathrm{Na}^{+}, \mathrm{K}^{+}, \mathrm{Cl}^{-}, \mathrm{HCO}_{3}{ }^{-}$, $\mathrm{SO}_{4}{ }^{-}{ }^{-}, \mathrm{NO}_{3}{ }^{-}$. (Tous les élêments dissous ne sont évidemment pas dosés, 
il manque notamment les formes du Fer, le Sr ( $1 \mathrm{mg} / 1)$, les nitrites, mais ils $\mathrm{n}^{\prime}$ interviennent que pour quelques $\mathrm{mg} / 1$ au total). La corrélation est bonne, compte tenu pour la Dranse des inflexions déjà citées pour les débits extrêmes. Ces relations du type $C_{d}=A Q_{1}{ }^{B}$ existent en fait pour toutes les rivières mais peu de données sont encore publiées. Nous donnons sur. 1a figure $\mathrm{II}_{10}$ une comparaison de six rivières de régimes hydrologiques différents, pour les débits allant de $50 \mathrm{l} / \mathrm{s}$ à $10000 \mathrm{~m}^{3} / \mathrm{s}$. L'établissement des bilans est donc très délicat. Il est évìdent que pour y parvenìr le plus grand nombre d'analyses doit être réalisé. Les méthodes classiques de calcul sont les suivantes :

1) $M=Q \cdot T \cdot \sum_{i=1}^{i=n} \frac{C i}{n}$

avec : M : masse de l'élément apportée pendant le temps T

$Q$ : débít moyen pendant $T$

Ci : concentration du prélèvement i

$\mathrm{n}$ : nombre de prélèvements pendant T

Cette façon de procéder est déjà fausse car elle ne pondère même pas les concentrations par les débits.

2) $M=\sum_{i=1}^{i=n} \frac{\text { Ci Vi }}{n}$

avec : Vi : volume moyen journalier du ième prélèvement

Cette méthode marque un progrès puisque les concentrations sont pondérées par les débits, néanmoins :

- 1a concentration peut varier pendant la journée.
- la méthode ne faît ìntervenìr que le débìt du jour du prêlèvement, des épisodes hydrologìques (crues ou étìages) peuvent être laissés pour compte sỉ le nombre des prélèvements n'est pas assez élevé compte tenu du régìme de la rivière.

I1 faut donc : - faire intervenir la variation des concentrations en fonction du débit.

- tenir compte des courbes de débits moyens classés suivant un intervalle de temps déterminé pour chaque rivière pendant lequel on peut considérer que le débit ne varie pas trop fortement. Cet intervalle sera donc fonction du régime hydrologique.

Nous avons donc cherché à satísfaire ces deux conditions :

- les variations des concentrations en fonction du débit ont été déterminées. Pour la plupart des éléments nous pourrons donc attribuer une concentration donnée à un débit quelconque. Pour les êlëments comme les chlorures et les nitrates seul un très grand nombre de prélèvements pourra donner des valeurs moyennes significatives, on calculera alors le bilan par la deuxième méthode.

- nous avons pris les débits moyens journaliers pour le Rhône et 1a Dranse. Cet intervalle de temps s'est avêré être tout juste suffisant pour la Dranse : il aurait sans doute fallu prendre les débits moyens sur $12 \mathrm{~h}$. Nous avons déterminé pour chaque rivière des classes de débits en progression logarithmique, découpant ainsi sur 1'axe des abscisses des courbes concentration - débit des intervalles égaux : 
Dranse : $Q<2,5 \mathrm{~m}^{3} / \mathrm{s} ; 2,5$ à $7 \mathrm{~m}^{3} / \mathrm{s} ; 7$ à $15 \mathrm{~m}^{3} / \mathrm{s} ; 15$ à $35 \mathrm{~m}^{3} / \mathrm{s}$; 35 à $70 \mathrm{~m}^{3} / \mathrm{s} ; 70$ à $120 \mathrm{~m}^{3} / \mathrm{s}$; > $120 \mathrm{~m}^{3} / \mathrm{s}$.

Rhône : $Q<60 \mathrm{~m}^{3} / \mathrm{s} ; 60-100 \mathrm{~m}^{3} / \mathrm{s} ; 100-160 \mathrm{~m}^{3} / \mathrm{s} ; 160-270 \mathrm{~m}^{3} / \mathrm{s}$; 270 à $450 \mathrm{~m}^{3} / \mathrm{s}$;

$\mathrm{Q}>450 \mathrm{~m}^{3} / \mathrm{s}$

Pour chaque année nous avons déterminé la répartition des débìts dans ces classes.

Deux méthodes de calcul sont alors possible.

\section{3) Méthode des concentrations moyennes pondérées.}

On détermine une concentration moyenne $C_{m}$ pondérée par les débits.

$C_{m}=\sum_{i} \alpha_{j} C_{j}$

avec $: C_{j}$ concentration moyenne correspondant à 1 'intervalle de débit $j$

$\alpha_{j}$ proportion de I'intervalle de débit $j$

puis $M=C_{m} Q \cdot T$

Bìen que faisant intervenir la variation des teneurs en fonction du débit, cette méthode est du même type que la première méthode. En effet, le débit le plus fréquent n'est pas nécessairement le débit moyen annuel.

\section{4) Méthode des bilans partiels.}

On effectue un bilan partiel pour chaque tranche de débits.
$M_{j}=Q_{j} \cdot T_{j} \cdot C_{j}$

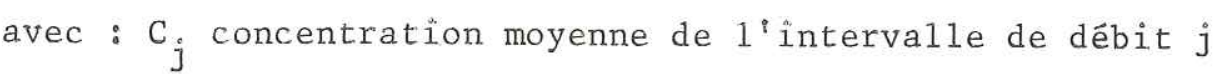

$Q_{j}$ débit moyen de l'intervalle j

Tj durêe totale des pérî̀odes de débîts comprìses dans 1'intervalle j

puis $M=\sum_{j} M_{j}$

Cette méthode nous semble la plus appropriée car elle tient compte à la fois de la répartition des débits et de la répartition de concentrations. La précision du bilan ainsi effectué dépend de l'intensité de la corrélation entre les concentrations et les débits, et du nombre d'intervalles de débits choisis. Cette méthode permet en outre de bonnes estimations des apports en se basant uniquement sur les débits pour autant que les corrêlations n'évoluent pas d'année en année.

\subsection{2. - Bilan des apports du Rhône.}

Nous avons effectué les bilans pour 1968 et 1969. Ces deux années ont été des années sèches : $166 \mathrm{~m}^{3} / \mathrm{s}$ et $157 \mathrm{~m}^{3} / \mathrm{s}$ alors que $1 \mathrm{e}$ module interannuel est de $180 \mathrm{~m}^{3} / \mathrm{s}$ (1935-1968). La répartition des débits est la suivante :

\begin{tabular}{|c|c|c|c|c|c|c|c|c|c|}
\hline & & $!$ & $!$ & $!$ & $!$ & $!$ & & $!$ & $T$ \\
\hline$!$ Dẻbit & ts $\mathrm{m}^{3} / \mathrm{s}$ & $\begin{array}{l}!<60 \\
!\end{array}$ & ! $60-100$ & $! 100-160$ & $! 160-2$ & & $270-450$ & $\begin{array}{l}! \\
!\end{array}>450$ & $!$ \\
\hline 1968 & & 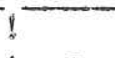 & $!$ & 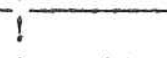 & $!$ & ! & & & \\
\hline ! nombre & de jours! & 0 & 44 & 162 & 122 & ! & 35 & $!$ & ! \\
\hline$!$ & $\%$ & 0 & 12 & $!$ & 34 & ! & 10 & $!$ & \\
\hline 1969 & & ? & 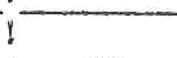 & 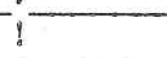 & $!$ & & & & 1 \\
\hline !nombre & de jours! & 7 & 82 & 146 & 86 & 1 & 43 & & \\
\hline & $\%$ & 2 & 23 & 40 & 23 & $P$ & 12 & $!$ & $!$ \\
\hline
\end{tabular}


TABLEAU II : $_{3}$ Bilan des apports en solution du Rhône

a) Méthode des concentrations moyennes pondérées.

- concentrations moyennes annuelles $C m=\sum_{j} \alpha_{j} C_{j}$, en $\mathrm{mg} / 1$.

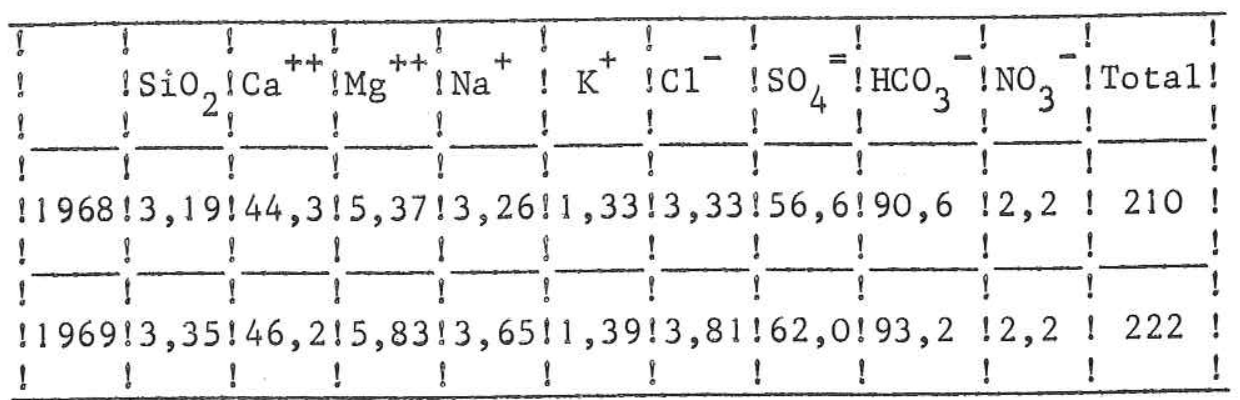

- apport annuel par élément $M=C m \cdot Q \cdot T$, en milliers de tonnes

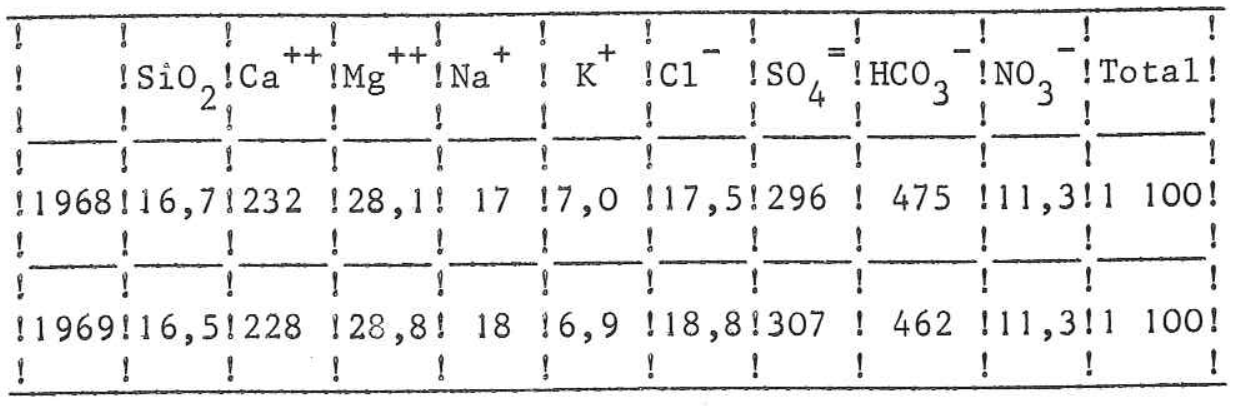

\section{b) Méthode des bilans partiels.}

- bilan de l'année 1968 en mïlilers de tonnes.

\begin{tabular}{|c|c|c|c|c|c|c|c|c|c|c|}
\hline $\begin{array}{c}\text { Classe de } \\
\text { débit }\end{array}$ & $\mathrm{SiO}_{2} !$ & $\mathrm{Ca}^{++}$ & $! \mathrm{Mg}^{++}$ & $\mathrm{Na}^{+}$ & $\mathrm{K}^{+}$ & $! \mathrm{Cl}^{-}$ & $!_{4} \mathrm{SO}_{4}=$ & $! \mathrm{HCO}_{3}{ }^{-}$ & $! \mathrm{NO}_{3}{ }^{-}$ & \\
\hline & & & $!$ & $!$ & & 8 & 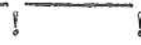 & & 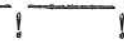 & \\
\hline $60 n$ & & & ! & & & & & & & \\
\hline & & & $!$ & $!$ & & & & & & \\
\hline $0=100 !$ &, 221 & & b! 2,40 & 11,67 & 0,48 & 1,83 & $3 ! 25,9$ & ! 31,6 & $5 ! 1,3 !$ & \\
\hline & & & $!$ & $!$ & $!$ & ! & $!$ & & $! \quad !$ & \\
\hline $00-$ & $15 !$ & 84,0 & ! 8 & & & & & & ! ! & \\
\hline & & & $!$ & $!$ & $!$ & & $!$ & & $!$ & \\
\hline $60-270 !$ & ! 6,77! & 92,5 & 110,2 & & & & & & & \\
\hline & & & 1 & $!$ & $!$ & $!$ & $!$ & & & \\
\hline $70-450 !$ & : 2,61! & 38,1 & $! 3,6$ & $! 1$ & & ! 1 & 13 & 18 & ! & \\
\hline & & & & & & & & & & \\
\hline & 0,01 & & & & & & & & & \\
\hline & & & & & & & & & & \\
\hline Total & , 8 & 23 & 124,4 & & 17 & & & & & \\
\hline
\end{tabular}

Ces résultats appellent plusieurs remarques :

- pour une même année, les deux méthodes donnent des résultats tout à faìt comparables compte tenu des nombreuses incertitudes commises.

- les bilans de 1968 et 1969 sont très proches, ce qui est normal compte tenu des débíts presque îdentiques et du mode de calcul.

- tous les éléments ont été déterminés d'après leur variation en fonction du débit sauf les nitrates pour lesquels nous avons pris une moyenne arithmétique annuelle. Les valeurs pour cet élément, mineur par rapport à la concentration globale mais important pour la pollution du lac, nous donnent juste un ordre de grandeur. Nous penson qu'un bilan significatif devrait être basé sur des analyses journalières. 

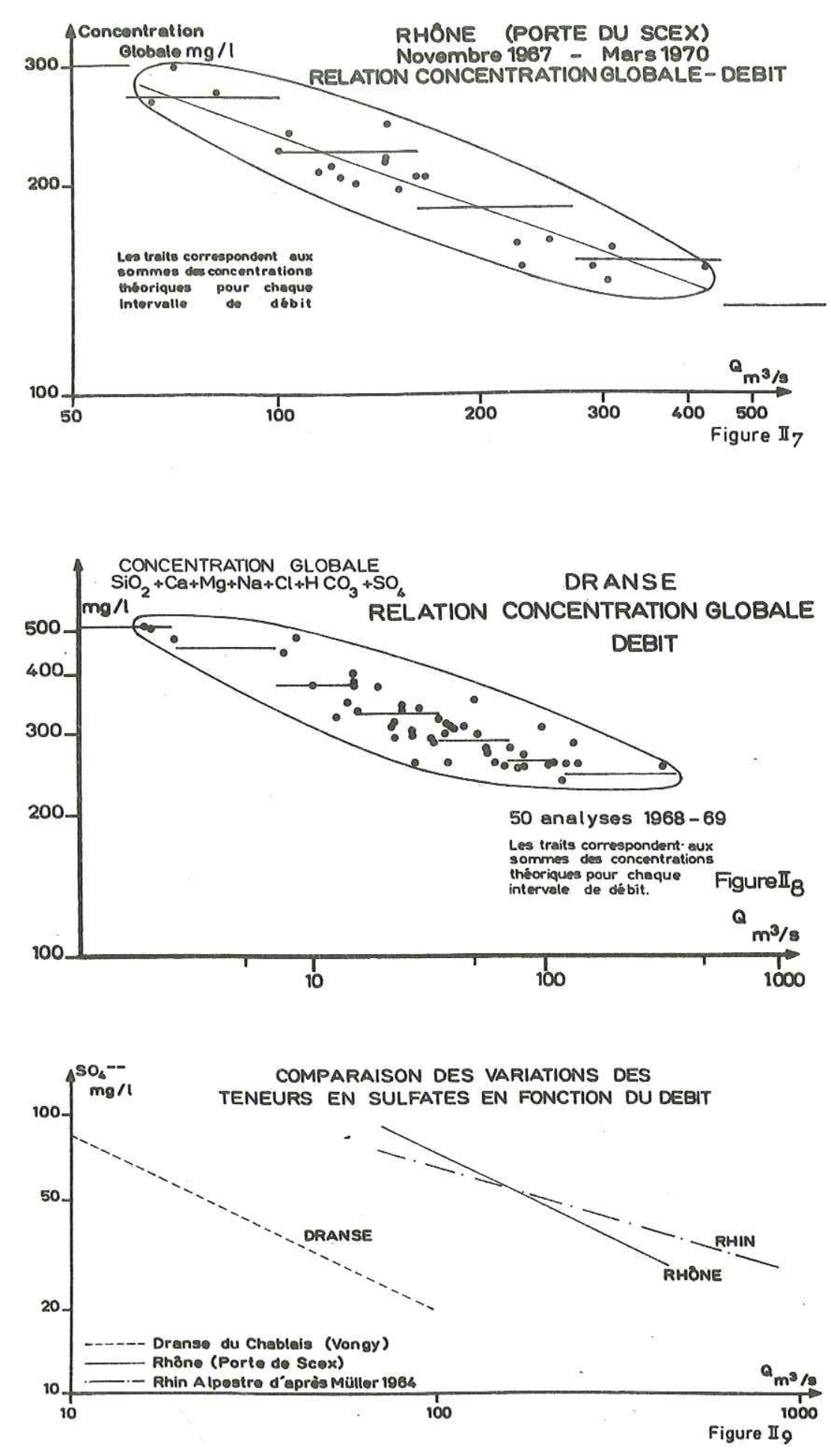

- la charge totale en éléments dissous est très légèrement inférieure à la réalité, en effet un certain nombre d'éléments mineurs et d'oligoéléments n'ont pas été dosés.

- nous avons portê sur la figure $\mathrm{II}_{7}$ les concentrations globales théorìques calculées en addîtionnant pour chaque intervalle de débìt les teneurs choììes pour chaque élément, on voìt que la correspondance est très satîsfaîsante.

Dussart (1961) donne par une méthode criticable un apport moyen annuel du même ordre : $1,23510^{6}$ tonnes en éléments dissous. Forel (Le Léman, tome I, p. 376) donnaît une valeur approxìmative de $0,9510^{6}$ tonnes, mais il semble que les débits choìsis étaient légèrement faïbles.

\subsection{3. - Bilan des apports de la Dranse.}

Nous ne disposons sur cette rivière que des débits classés pour 1968 (année moyenne de débìt $23,7 \mathrm{~m}^{3} / \mathrm{s}$ ).

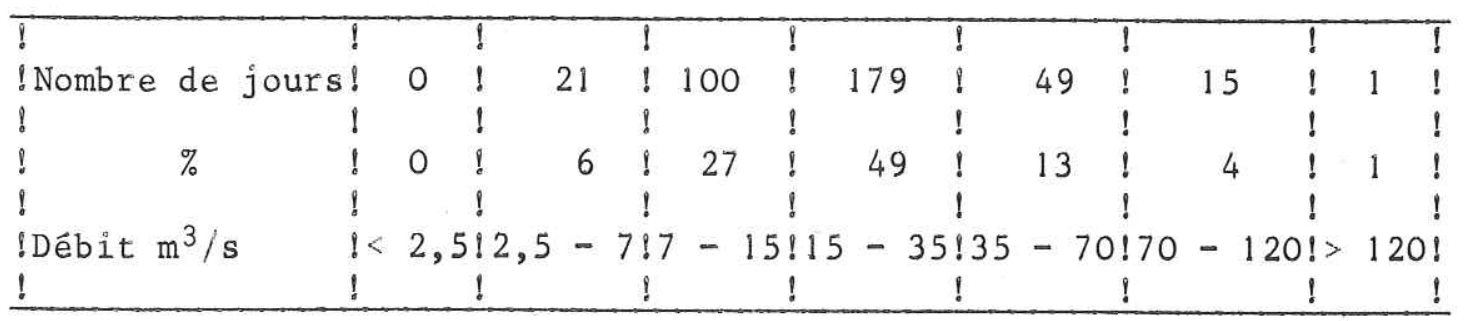


TABLEAU $\mathrm{II}_{4}$ : Bilan des apports en solution de la Dranse pour 1968

a) Méthode des concentrations moyennes pondérées.

Concentrations moyennes annuelies $\mathrm{Cm}=\sum_{j} \alpha_{j} C_{j}$, en mg/1.

Apport annue1 par élément $M=C m Q$ 。 tonnes.

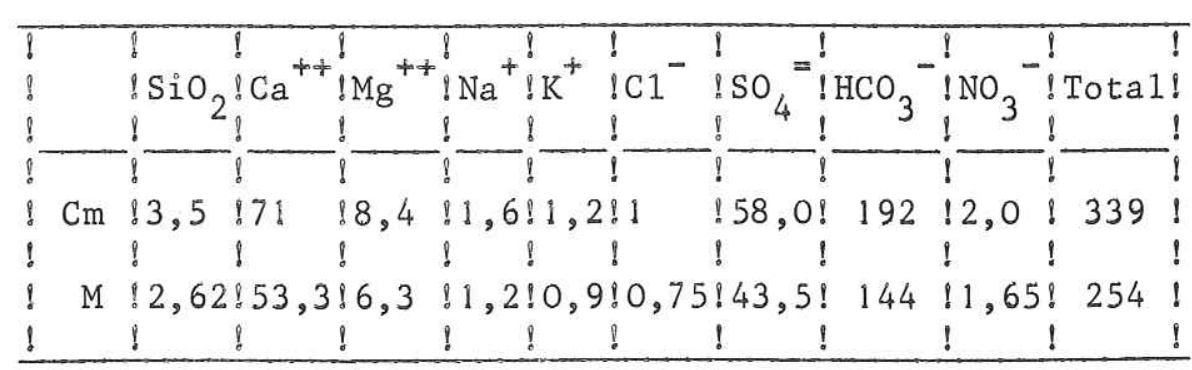

b) Méthode des bilans partiels en milliers de tonnes.

\begin{tabular}{|c|c|c|c|c|c|c|c|c|c|}
\hline $\begin{array}{l}\text { Classe de } \\
! \\
!\end{array}$ & $\mathrm{Ca}^{++}$ & $!^{\mathrm{Mg}^{++}}$ & $\mathrm{Na}^{+}$ & $\mathrm{K}^{+}$ & $\mathrm{Cl}^{-}$ & $\mathrm{SO}_{4}=$ & $! \mathrm{HCO}_{3}^{-}$ & $!_{\mathrm{NO}_{3}}^{-}$ & $\begin{array}{l}! \\
! \\
!\end{array}$ \\
\hline 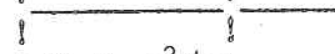 & 1 & ! & $!$ & $!$ & & & & $!$ & 1 \\
\hline$!<2,5 \mathrm{~m}^{3} / \mathrm{s} !$ & $!$ & ! & ! & ! & ! & & ! & & \\
\hline ! & $!$ & $!$ & $!$ & $!$ & $!$ & & $!$ & $!$ & $!$ \\
\hline $7 ! 0,03 !$ & 0,85 & $5 ! 0,117$ & 10,022 & $2 ! 0,011$ & :0,009! & 1,17 & 1,89 & 910,018 & $4,13 !$ \\
\hline$!$ & $!$ & $!$ & $!$ & $!$ & $!$ & & $!$ & $!$ & $!$ \\
\hline $7-15 ! 0,332$ & $2 ! 7,60$ & 10,950 & 0,190 & $0,0,114$ & 40,095 ! & ! 7,60 & 19,0 & 10,190 & $0 ! 36,1$ \\
\hline 1 & $!$ & $!$ & $!$ & $!$ & $!$ & & & & \\
\hline $35 ! 1,35$ & $! 27,0$ & 13,09 & $! 0,617$ & $7 ! 0,462$ & $2 ! 0,386$ & $! 19,3$ & 173,3 & 10,772 & $2 ! 128,1$ \\
\hline 1 & $!$ & $!$ & $!$ & $!$ & $!$ & & $!$ & & $!$ \\
\hline $70 ! 0,738$ & $3 ! 13,7$ & 11,265 & $5 ! 0,253$ & $3 ! 0,2$ & $3 ! 0,211 !$ & ! 6,33 & $! 37,9$ & 10,422 & $2 ! 61,2$ \\
\hline & $!$ & $!$ & 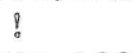 & $!$ & $!$ & & $!$ & & $!$ \\
\hline $70-120 ! 0,427$ & ! 6,71 & 10,610 & 10,122 & 210,146 & $5 ! 0,122$ & ! 2,44 & ! 21,35 & $5 ! 0,242$ & $2 ! 32,2$ \\
\hline 1 & $!$ & & $!$ & 1 & $!$ & & $!$ & & \\
\hline $20: 0,042$ & $=0,60$ & 10,054 & $4 ! 0,010$ & $2 ! 0,014$ & $4 ! 0,012$ & 0,180 & 2,04 & $4 ! 0,02$ & 2,97 \\
\hline Total !2,9 & $\$ 56,5$ & $! 6,08$ & $! 1,21$ & $! 1,0$ & $! 0,83$ & $! 37,0$ & $! 155,6$ & $! 1,67$ & $! 265$ \\
\hline ! & & $\vdots$ & & & & $!$ & & & $!$ \\
\hline
\end{tabular}

Comme pour 1.e Rhône les chlorures et les nitrates sont donnés à titre indicatif, la précision étant faible. Nous avons porté sur la figure $\mathrm{II}_{8}$ les concentrations globales théoriques comparées à l'enveloppe des concentrations mesurêes. Comme pour le Rhône la correspondance est très satisfaisante,

\section{6. - Erosion des bassins versants par dissolution.}

\subsection{1. - Erosions spécifiques pour chaque élément.}

Nous donnons dans le tableau suivant les analyses types du Rhône et de la Dranse, obtenues en divisant les apports annuels calculês précédemment par le débit moyen annuel; nous avons alors pu calculer les taux d'érosion spécifique. Nous avons joint au tableau les données calculées pour le Rhin d'après les chiffres de Müller (1964). Remarquons que, sì on considère la courbe de frêquence des concentrations sur un an, cette concentration moyenne définie plus haut est une moyenne pondérée par les débits qui diffère du mode (concentration la plus fréquente) de la médiane (concentration correspondant à $50 \%$ dans la courbe des fréquences cumulées) et de la moyenne arithmétique.

TABLEAU II $_{5}$ : Erosions spêcifiques chimiques du Rhône, de la Dranse et du Rhin Alpestre

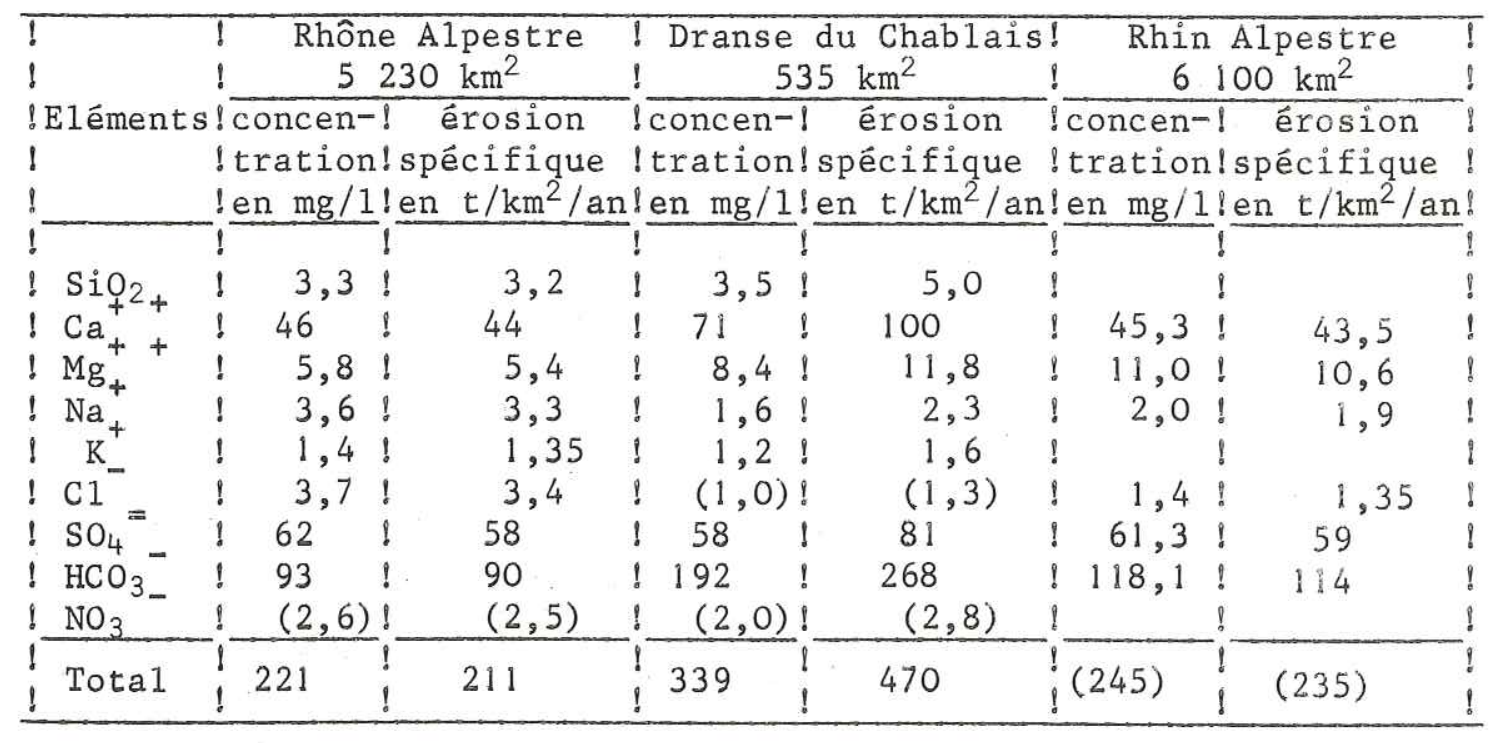


L'érosion spécifique globale a été extrapolée pour le Rhin. Plusieurs remarques s'imposent :

- il y a une grande similitude entre le Rhône et le Rhin tant par la composition chimique très voisine que par les valeurs des érosions spécifiques. Ces deux rivières ont en effet des caractêristiques hydrologiques très semblables, de plus la nature lithologique de leur bassin versant est analogue. Les travaux de Müller (1964) montrent une variation identique de la plupart des éléments en fonction du débit. Mưller donne en gếnéral les valeurs moyennes mensuelles mais pour les sulfates nous disposons des concentrations et des débits instantanês pour chaque prélèvement, nous avons comparé cette courbe à celle de la Dranse et du Rhône sur la figure IIg. Une fois encore le Rhin et le Rhône ont un comportement analogue.

Les valeurs des érosions pour 1a Dranse sont nettement plus fortes : en effet le débít spécifique est supérieur à celui du Rhône $\left(43,4 \mathrm{I} / \mathrm{s} / \mathrm{km}^{2}\right.$ contre $\left.34,61 / \mathrm{s} / \mathrm{km}^{2}\right)$

- ces valeurs d'érosions sont à comparer à celles calculées pour 1a Loire d'après les données de Berthois (1969) pour un bassin versant de $110000 \mathrm{~km}^{2}$, et un débìt spécìfique de $7,91 / \mathrm{s} / \mathrm{km}^{2}$.

\begin{tabular}{|c|c|c|c|c|c|c|}
\hline & $\varliminf_{2}$ & $! \mathrm{Ca}^{++}$ & $! \mathrm{Mg}^{++}$ & $! \mathrm{Na}^{+}$ & $\mathrm{x}^{+}$ & $\begin{array}{c}\text { Total des } \\
\text { éléments dissous }\end{array}$ \\
\hline $\begin{array}{l}\text { Erosion } \\
\mathrm{t} / \mathrm{km}^{2} / \mathrm{an}\end{array}$ & 3,36 & 6,14 & 2,71 & 2,36 & $6 ! 0,70$ & 42 \\
\hline $\begin{array}{l}\text { Concentration } \\
\text { mg/1 }\end{array}$ & $!$ & $! 24,6$ & $! 10,9$ & $! 9,5$ & $! 2,8$ & 169 \\
\hline
\end{tabular}

On voit que sur $1^{\prime}$ ensemble d'un grand bassin 1'érosion spécifique diminue considêrablement. Pour certains auteurs le débit spécifique jouerait un rôle important. Nous verrons au quatrième chapitre (figure $I V_{6}$ ) que cette corrêlation $n^{\prime}$ est pas encore bien établie :

il faudrait disposer d'un grand nombre de résultats sur des bassins versants de tailles, d'altitudes, et de climats diffêrents.

\subsection{2. - Cas de la silice.}

- Une foìs de plus il est à remarquer le comportement tout à fait à part de la silîce dont l'érosion spécifíque ne semble pas varier avec la taille des bassins étudiés. Pour Corbel (1957) la mise en solution de la sìlice est essentìellement lìée au climat. Il donne, dans l'unité peu pratîque de mm d'érosion de sillicates pour 1000 ans (1), les valeurs suivantes :

Climats artiques a étê chaud

Climats continentaux type Oregon

Climats tempérés océanìques

Climats tropicaux à période sèche

Climats équatoriaux (Amazone) $2,6 \mathrm{~g} / \mathrm{cm}^{3}$ nous trouvons les valeurs suivantes :
Dranse du Chablais

Rhône Alpestre

Loire
$0,8 \mathrm{~mm} / 1000$ ans

$5 \mathrm{~mm} / 1000$ ans

$0,9 \mathrm{~mm} / 1000$ ans

0,1 à $4 \mathrm{~mm} / \mathrm{l} 000$ ans

4 à $9 \mathrm{~mm} / 1000$ ans
Pour Davis (1964) par contre le taux d'érosion de la silice est directement lié à la lame d'eau écoulée sur le bassin versant. I1 donne pour des bassins divers répartis sur tout les Etats-Unis, la relation entre la lame d'eau en cm/an et l'érosion de la silice en $\mathrm{mg} / \mathrm{cm}^{2} / \mathrm{an}$. Nos résultats s'exprìment alors de la façon suivante : 


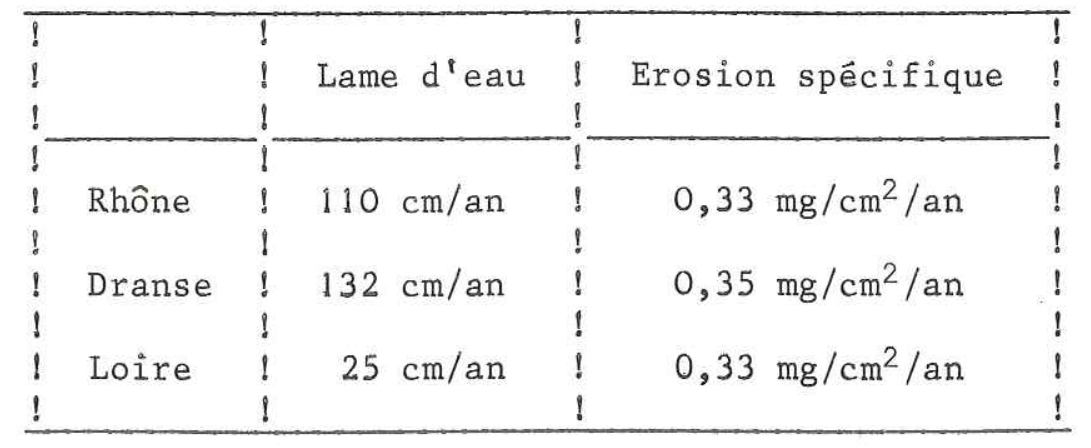

Il semble bien que 1 'hypothèse de Corbel soit la plus valable si on considère ces trois rivières, d'autant plus que nous avons vu que 1a silice provient surtout du lessivage des sols et non pas des eaux souterraines. De nombreuses analyses sur d'autres rivières diffêrentes seraient nécessaires pour pouvoir vraiment trancher.

\subsection{3. - Conclusions.}

Nous avons vu que, pour une même rivière, les concentrations en éléments dissous étaient très variables. Les causes en sont multiples mais le phénomène essentiel reste $1 \mathrm{a}$ variation des concentrations en fonction du débit. Nous avons reporté cette variation sur la figure $\mathrm{II}_{10}$ : pour des rivières très différentes $1 \mathrm{e}$ phénomène est partout le même. C'est seulement aprẽs avoir étudié le phénomène principal qu'on peut aborder les facteurs secondaires (saisons, épisodes hydrologiques etc...). Le comportement des différents éléments en solution donne alors des renseignements précieux sur leur origine probable (eaux souterraines, eaux superficielles, pollutions, précipitations atmosphériques). Enfin, à moins de disposer de mesures continues des concentrations, il est indispensable de déterminer d'abord les causes de leurs variations afin de trouver les modes de calcul des bilans les plus appropriés.

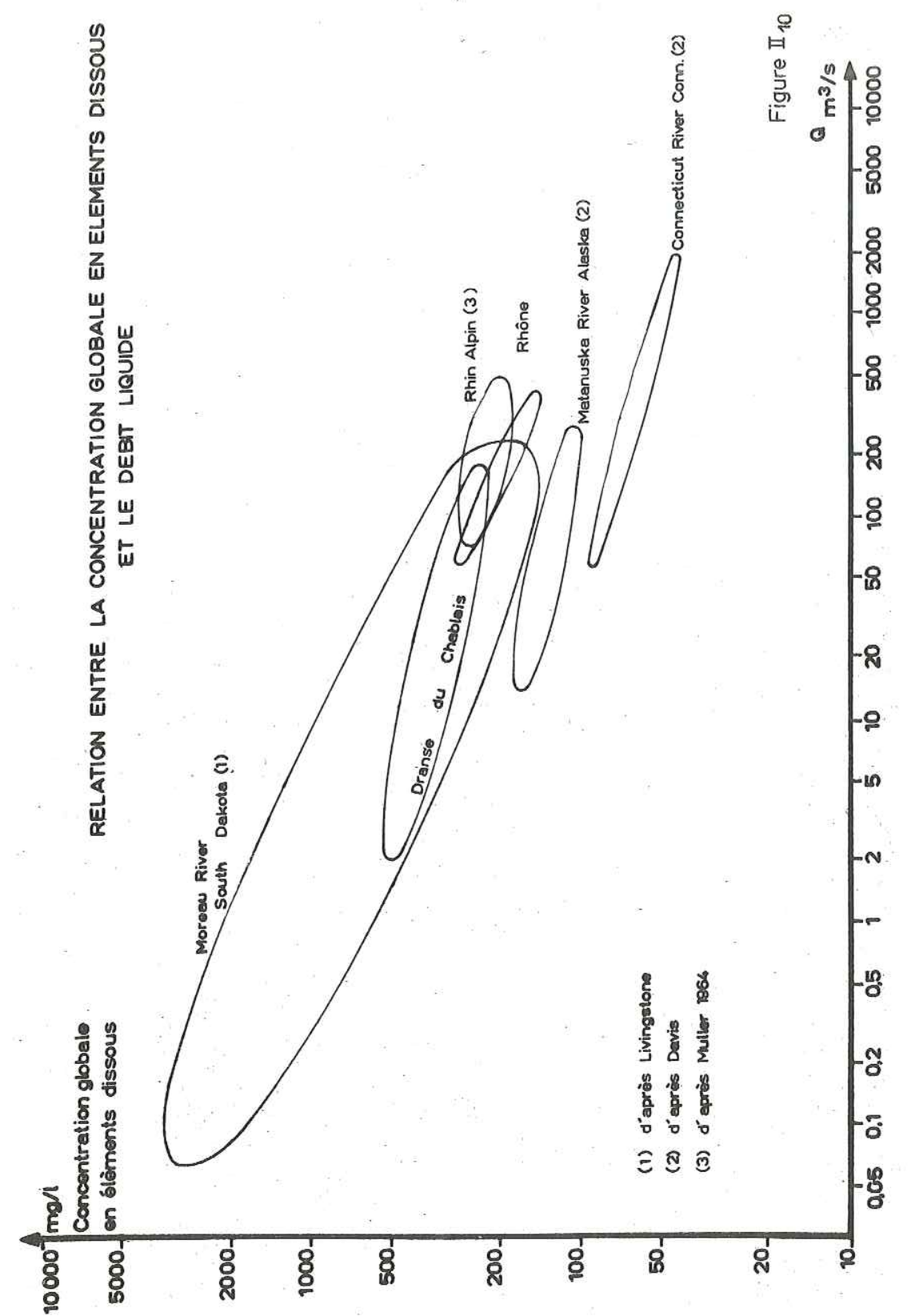




\begin{tabular}{|c|c|c|c|c|c|}
\hline 1 & & 墨 & & 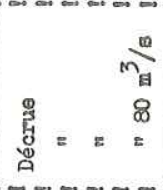 & 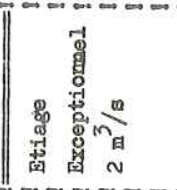 \\
\hline . & & & $\stackrel{\infty}{\circ}$ & 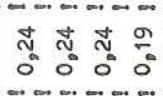 & $40_{0}^{\prime}$ \\
\hline 8 & 18 & 15020 & 望 & 98 & $108 \overline{8}$ \\
\hline : & $=07$ & 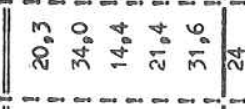 & 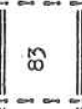 & 8050 & 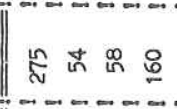 \\
\hline 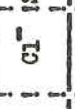 & 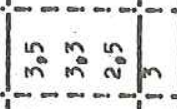 & $\left\{\begin{array}{l}0 \\
80\end{array}\right.$ & -1 & 25 & $\therefore=$ \\
\hline \pm & $\mid$ & 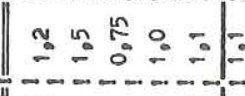 & 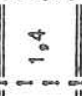 & 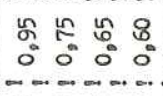 & 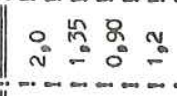 \\
\hline 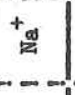 & $\begin{array}{lll}0 & 8 & 0\end{array}$ & 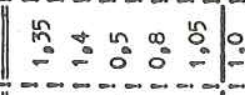 & $=1$ & 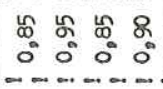 & 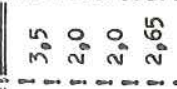 \\
\hline . & 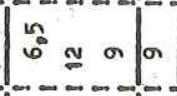 & 1 & $\left.\mid \begin{array}{ll}0 \\
0 \\
0\end{array}\right]$ & 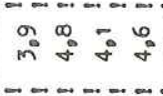 & 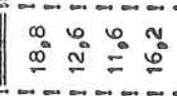 \\
\hline$f_{s}$ & $98 \mathrm{~N}$ & $\mid \begin{array}{lll}0 \\
0 \\
1085\end{array}$ & $:$ & 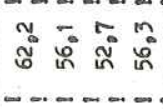 & $8 \approx 80$ \\
\hline | & & & $=$ & 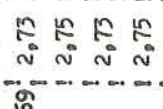 & $\frac{1}{2}=0^{2}=0$ \\
\hline 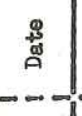 & 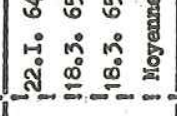 & 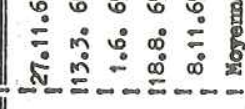 & 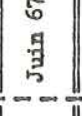 & 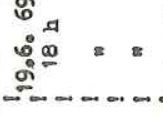 & 恶 \\
\hline 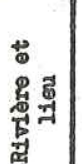 & & 睊高 & : & 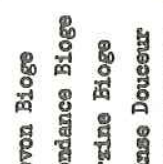 & 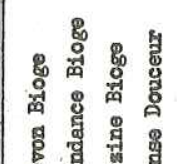 \\
\hline 8 & & & & & \\
\hline 1 & & 18 & 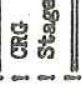 & 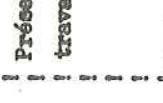 & In \\
\hline
\end{tabular}

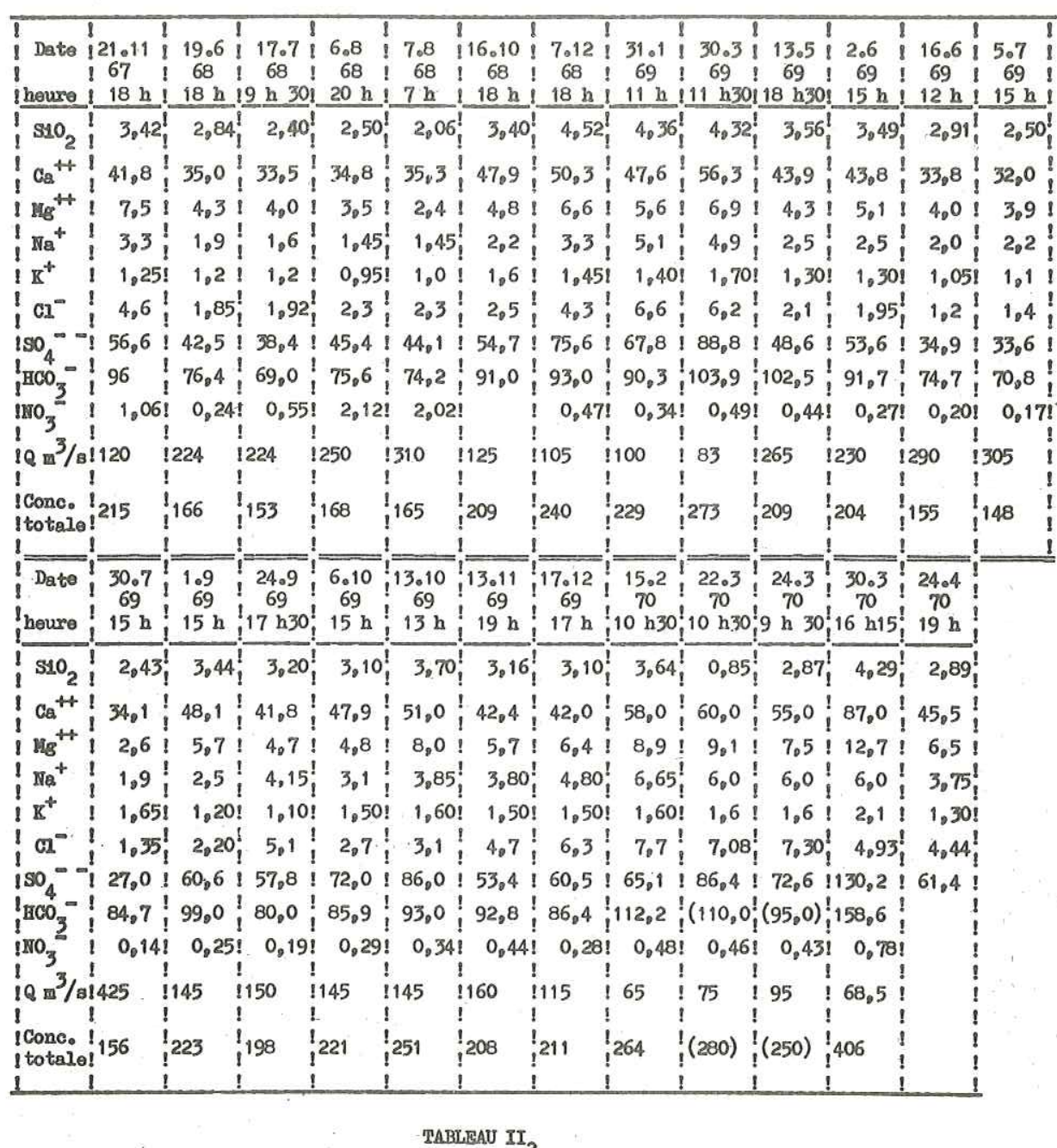

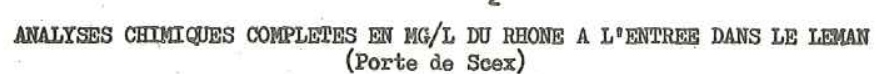

Remaravoes : - ( ) Valeurs extrapolíos 


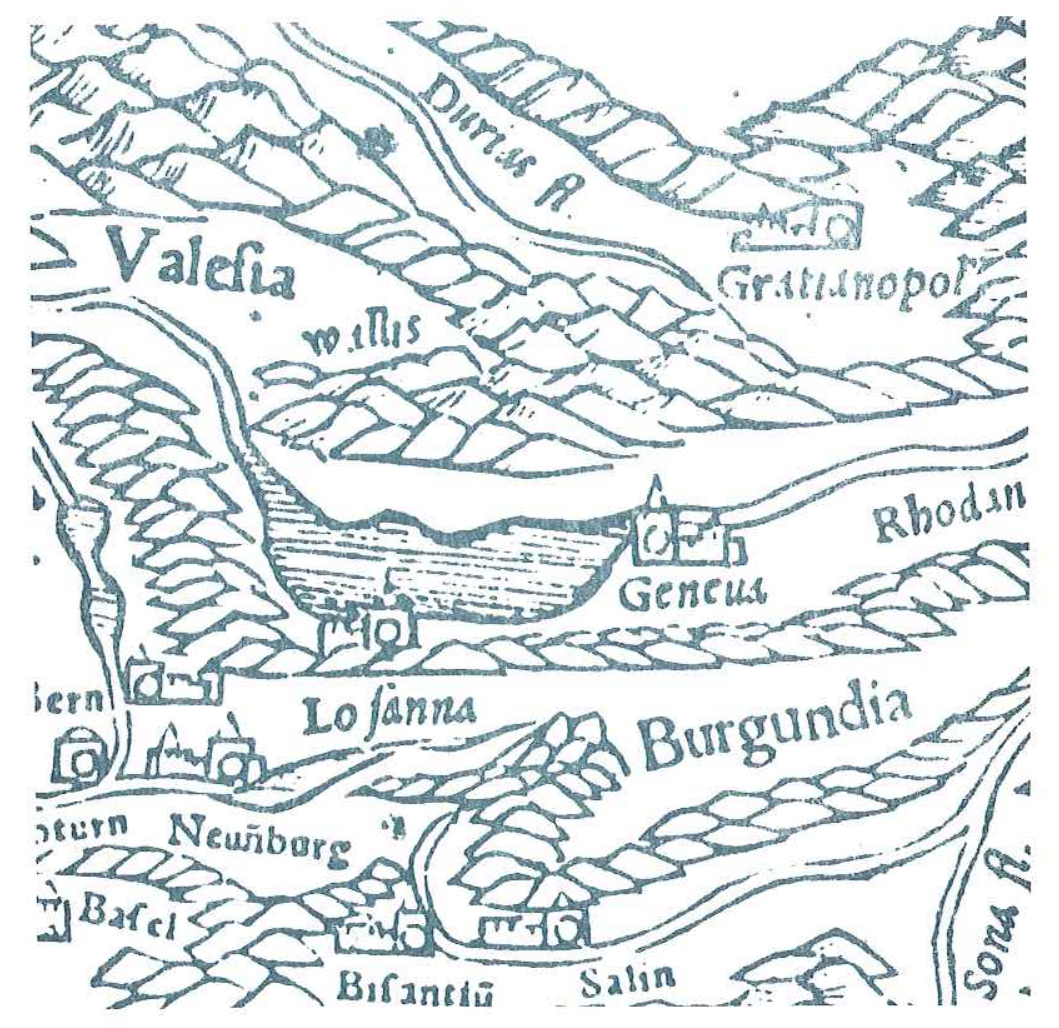

COMPOSITION CHIMIQUE DES EAUX DU LEMAN ET

BILAN DES ELEMENTS DISSOUS 


\section{A - COMPOSITION CHIMIQUE DES EAUX DU LAC.}

3.1. - Histoire de I'étude chimique des eaux du Léman et position du problème.

Le Léman a attirế depuis très longtemps 1 'attention des analystes : Forel cite une analyse de Tingry effectuée en 1808 et exprimée en mesures royales. Jusqu'à Forel et Delebecque aucune étude globale des phénomènes chimiques dans le Léman n'est réalisée. A quelques années d'intervalle les deux chercheurs formulent des hypothèses contradictoires. Pour Delebecque (les lacs français, p. 211) la composition de l'eau est variable suivant la profondeur et ces variations s'observent essentiellement sur la silice et les carbonates, de plus l'action des affluents ne se fait sentir qu'à une faible distance de $I^{\prime}$ 'embouchure. Pour cet auteur $I^{\prime}$ origine des variations est essentiellement chimique sur les carbonates et biologique sur la silice. Cette étude déjà très poussée est encore complétée par des considérations sur l'émissaire : 1'eau de l'émissaire a une composition voisine de celle des eaux de surface et non pas une composition moyenne des eaux du lac.

Pour Fore1 (Le Lêman, tome II, p. 575) par contre, 1a composition des eaux doit être invariable : les seules causes pouvant entrainer une modification des eaux sont les affluents. Le savant vaudois se base sur la constatation suivante : pour qu'il y ait une variation d'l $\mathrm{mg} / 1$ dans 1 a totalité du lac il faut un apport de $89000 \mathrm{t}$, or il n'y a pas d'agent interne ou externe qui puisse faire varier ces 
proportions d'un jour à l'autre ou d'une année à l'autre. Forel ne croit pas à la précipitation chimique ("variation très faible") quant à 1'influence des organismes biologiques elle n'a pas d'"action sensible sur les eaux du lac". Quoíqu'il en soit "les actions très faibles Q'altêration que subîssent les eaux du lac pendant leur séjour dans le Léman se font sentir uniquement dans les eaux de surface". Cependant à la fin de son ouvrage Forel reverra sensiblement sa position, les travaux de Delebecque étant parus entretemps. Enfin, pour ces deux auteurs les eaux sont entièrement homogènes en hiver.

D'autres auteurs ont donné depuis des analyses chimiques du lac, citons : Zender (1908), Dienert (1913) et Hubault (1947). En 1925 Balavoine à la suite d'une très intéressante étude poursuivie sur trois ans,montra pour la première fois la variation saisonnière de la composition globale du Petit Lac, notamment des carbonates et des sulfates. Mais il faudra attendre 1954 pour que l'étude approfondie et détaillée de la chimie du lac soit réalisée par Monod (1956). Cet auteur a étudié un grand nombre de paramètres physico-chimiques et d'éléments dissous à 1 'embouchure du Flon à Lausanne. Pour la première fois les phénomènes entrevus par Delebecque sont analysés, chiffres à 1'appui, citons notamment : 1a précipitation des carbonates, le cycle de l'oxygène, la variation des phosphates et des composés azotés. Néanmoins 1'étude de Monod reste très locale : il s'agit d'une zone de $1 \mathrm{~km}$ de diamètre à l'embouchure d'une petite rivière, avec des profondeurs maximales de $32 \mathrm{~m}$. Il restait donc de nombreuses incertitudes et les variations géographiques, saisonnières ou suivant la profondeur, de la composition chimique dans l'ensemble du lac étaient encore mal connues. Notons que pour Monod (1960) également "en hiver les eaux se mélangent complètement de la surface jusqu'au fond, c'est la période de circulation".

En 1957 fut créée la "Commission Internationale pour la protection des eaux du Léman et du Rhône contre la pollution". Cette organisme, dont le Centre de Recherches Géodynamiques fait partie depuis 10 ans, comprend des laboratoires suisses et français. Des prélèvements et des analyses réguliers sont effectuês sur toute l'étendue du Léman et sur les affluents dans les domaines suivants : physicochimie et chimie des eaux, microbiologie, bactêriologie, biologie. Depuis près de quinze ans une somme énorme de résultats a été amassée, dont une partie seulement a été dépouillée, nous verrons plus loin tout 1 'intérêt d'une telle étude. Au point de vue du chimisme général du Lẻman les résultats de la Commission n'ont pas été exploités, de plus plusieurs éléments principaux n'ont pas été dosés dans tout le lac (silice, sulfates). Avant d'étudier le bilan géochimique du bassin du Léman, il était donc essentiel d'essayer d'apporter des réponses aux questions suivantes :

- Y a-t-il des variations importantes des éléments entre la surface et le fond du lac ?

- Y a-t-il des variations géographiques entre les différentes régions du 1 ac et quelle est 1 'influence chimique des affluents ?

- Y a-t-il des variations saisonnières générales pour l'ensemble du lac et quels sont les éléments affectés?

- Comment la composition de l'eau de sortie du lac se situe t-elle par rapport à l'ensemble du Léman ?

- Est-il possible de trouver un élément dissout qui puisse servir de repère ou de traceur pour l'étude du mouvement des eaux ?

- Y a-t-il véritablement une période de circulation avec une homogénéisation globale des eaux ?

Enfin rares sont les lacs dont l'étude a commencé avant 1900 et ce n'est pas $1^{\prime}$ un des moindres mérites des travaux de Forel et de Delebecque de nous donner avec précision l'état du Léman vers 1880. Nous essayerons de voir quels sont les changements apportés à la composition chimique des eaux par l'industrialisation et le peuplement massifs de son bassin versant depuis cette époque : y a-t-il une véritable pollution chimique comme dans certains lacs du St Laurent ou bien une eutrophisation surtout biologique 
3.2. - Prélèvements et analyses.

\subsection{1. - Points de prélèvements du C.R.G.}

Les points réguliers sont portés sur la figure $\operatorname{III}_{1}$. II s'agit des anciens points de prélèvement de la Commission de Protection du Lêman (C.R.G.3, 4, 6 et 9) auxquels nous avons ajouté en 1968 trois nouveaux points destinés à étudier 1 'influence sur le lac des deux principales rivières, le Rhône et la Dranse. En plus de ces stations régulières un certain nombre d'autres prélèvements ont été réalisés soít dans la zone de la Dranse, soit dans celle du Rhône, ou au point C.R.G. 10 au centre du lac. Les coordonnées exactes des points sont données dans le tableau III $_{1}$.

TABLEAU III $_{1}$ : Coordonnées des stations de prélèvements hydrologiques

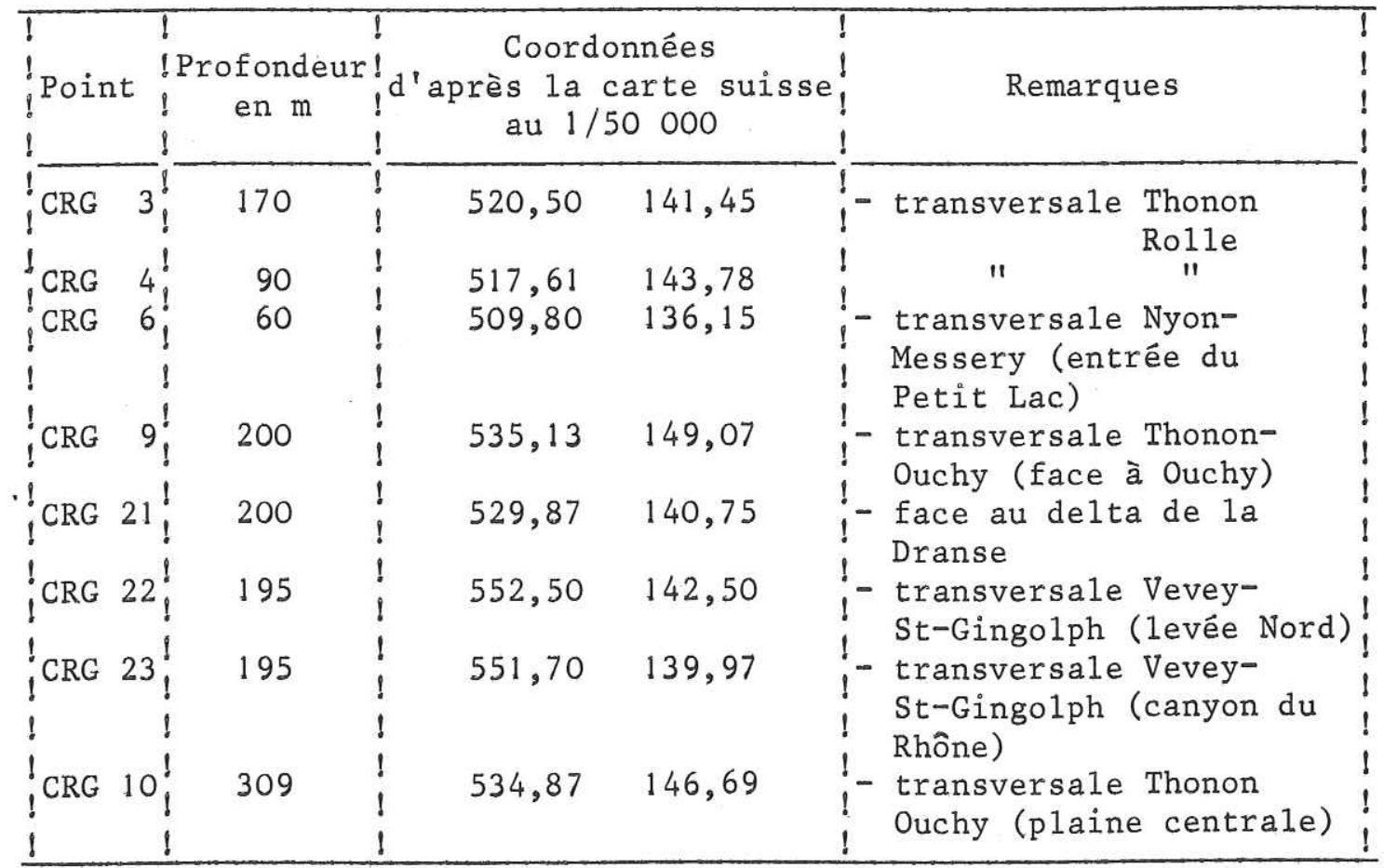

STATIONS HYDROLOGIQUES SUR LE LEMAN
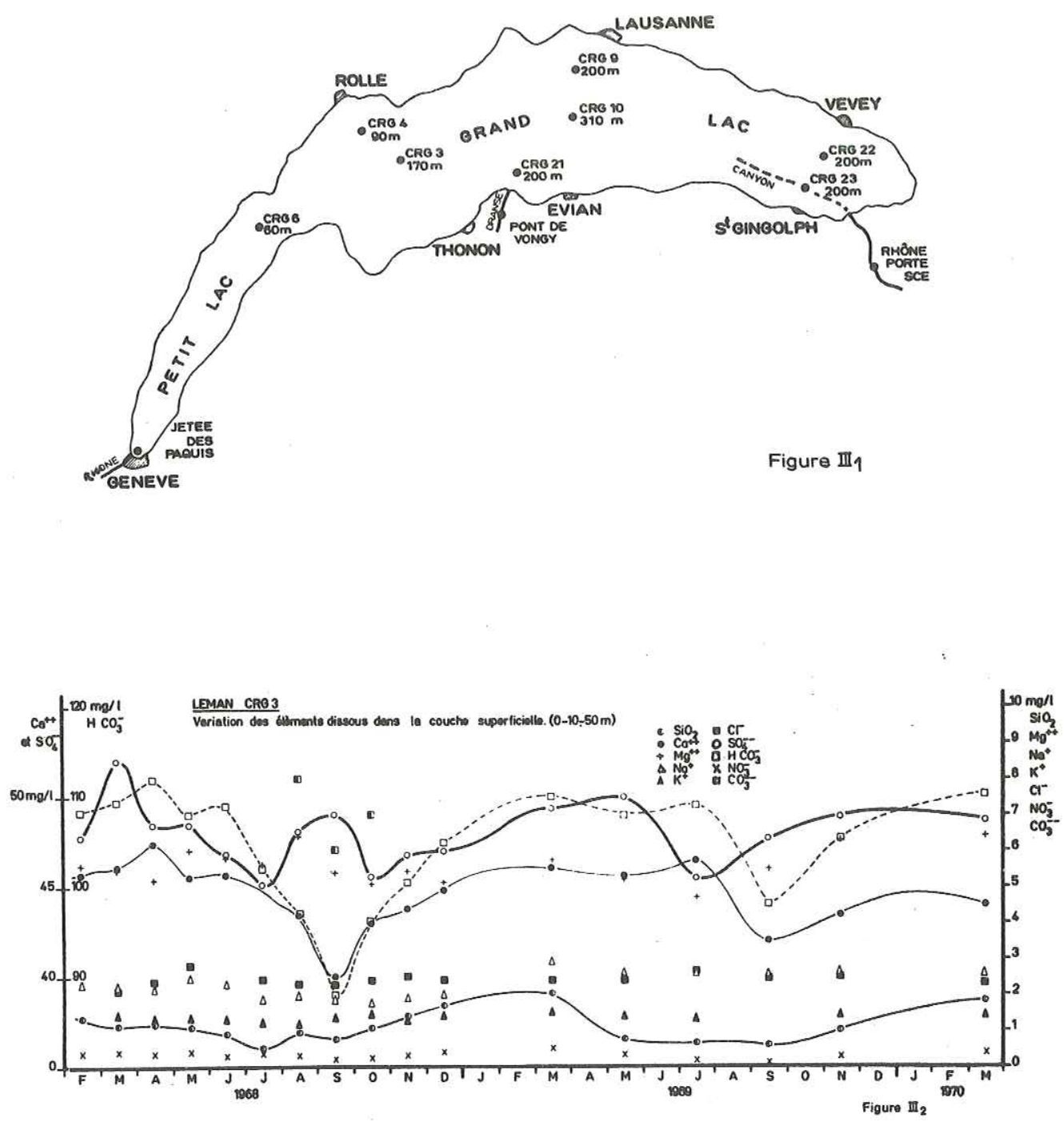
Remarquons que le point CRG 23 est situé dans le canyon actuel du Rhône et que le poìnt CRG 22 se trouve sur 1a levée Nord de ce canyon à la même profondeur. Enfin il est à regretter que notre programme de prélèvement déjà chargé ne nous ait pas permis de suivre régulièrement l'évolution du poìnt CRG 10, seules des études particulières ont pu être rểalisées, notamment pour la silice.

\subsection{2. - Fréquence des prélèvements.}

L'étude proprement dite a commencé en Février 1968, pour cette année là les prélèvements ont généralement eu lieu vers le milieu de chaque mois. Pour 1'année 1969 la fréquence a été mensue1le seulement pendant 1'été (mars, mai, juillet, août, septembre, novembre). En 1970 les campagnes ont été trimestrielles, nous verrons plus loin que les variations du lac sont surtout saisonnières, un tel programme donne donc déjà une idée qualitative satisfaisante des phénomènes.

\subsection{3. - Profondeurs des prélèvements.}

Vus les travaux antérieurs, surtout ceux de Monod, et les études isotopiques entreprises au C.R.G. depuis 1964, nous avons prêlevé en surface et à $10 \mathrm{~m}$ afin d'étudier plus précisément la zone supérieure soumise à de fortes variations physicochimiques et biologiques. Ensuite les prélèvements ont été effectués à 50,100,150 m, $200 \mathrm{~m}, 250 \mathrm{~m}$ et $300 \mathrm{~m} \mathrm{~s}$ 'il y avait lieu. Pour 1'oxygène et quelquefois pour la silice, des prélèvements intermédiaires ont été réalisés à 20,30 et $40 \mathrm{~m}$.

\subsection{4. - Eléments étudiés.}

Les éléments dissous étudiés sont les mêmes que pour les rivières : $\mathrm{Ca}^{++}, \mathrm{Mg}^{++}, \mathrm{Na}^{+}, \mathrm{K}^{+}$et $\mathrm{Cl}^{-}, \mathrm{SO}_{4}^{--}, \mathrm{HCO}_{3}^{-}, \mathrm{CO}_{3}^{--}, \mathrm{NO}_{3}^{-}$ et la silice. A chaque campagne les mesures de $\mathrm{pH}$ par colorimétrie, de température à l'électrothermomètre et par thermomètre à renversement mécabollier, et de transparence au disque de Scechi ont été effectuées sur place aînsì que la fixation de l'oxygène par la méthode de Winkler. Les méthodes d'analyses en laboratoire sont les mêmes que celles utilisées pour les eaux des rivières. Il est important de noter que les éléments étûdiés concernent plus de $99 \%$ de la concentration globale en éléments dissous (voîr tableau III $_{11}$ ). L'erreur commise en assimilant la concentration globale à la somme de ces 10 éléments est donc faíble.

\subsection{5. - Problèmes de méthologie.}

Quand on aborde 1'étude d'un lac comme le Léman il se pose la question très importante de l'échantillonnage : faut-il multiplier les profondeurs ou les points de prélèvements ou encore faut-il augmenter la fréquence des campagnes ? Comment faut-il échantillonner 1'émissaire ? Les réponses à ces questions dépendent évidemment du type de variations de la composition chimique des eaux, nous pourrons essayer d'y répondre après avoir étudié les différentes variations : géographiques, saisonnières et suivant les profondeurs.

Compte tenu de nos derniers travaux d'hydrologie isotopique, nous avons, dans ce travail, divisé le lac en quatre couches : couche supérieure 0-60 m correspondant aux prélèvements de 0-10 et $50 \mathrm{~m}$; couche moyenne de $60 \mathrm{~m}$ à $180 \mathrm{~m}$ (100 et $150 \mathrm{~m}$ ) ; couche intermédiaire $180 \mathrm{~m}$ à $220 \mathrm{~m}(200 \mathrm{~m})$ et couche profonde de $220 \mathrm{~m}$ à $310 \mathrm{~m}$. Cette division a été rendue nécessaire pour le calcul de la teneur moyenne du lac en tenant compte des volumes partiels de chaque couche.

Dans la plupart du temps nous avons comparé les valeurs moyennes des concentrations globales ou partielles par point, par couche ou par mois ; les fluctuations dues au simple hasard laissent ainsi place aux phénomènes principaux. Nous avons reporté toutes ces moyennes dans de nombreux tableaux. Seules les analyses de l'exutoire 
du 1 ac à Genève sont reportêes intégralement au tableau III $I_{2}$. II n'était en effet pas possible de reporter ici l'intégralité de plus de 700 analyses chimiques complètes quì ont été réalisées sur le Léman en deux ans et demí au Centre de Recherches Géodynamiques.

Enfin nous nous sommes particulièrement attachés à 1 'étude du point CRG 3. Ce poìnt, situé au mìlìeu du lac entre Thonon et Rolle par $170 \mathrm{~m}$ de fond, représente assez bien 1 'ensemble du Léman (rappelons que la profondeur moyenne du Grand Lac est de $172 \mathrm{~m}$ ). Nous donnerons donc pour ce poînt des données physicochimiques complètes en plus des variations chimiques pour chaque élément.

\section{3. - Variabilité de la composition chimique.}

\subsection{1. - VaLeurs extrêmes du Léman.}

Nous avons recherché pour les points principaux et pour l'ensemble du lac, les valeurs extrêmes observées de Février 1968 à Septembre 1970 (tableau III $_{3}$ ). Les premières mesures de Novembre 1967 ont étế incluses dans cette recherche. Les quatre éléments les plus variables ont étê étudiês : $\mathrm{HCO}_{3}{ }^{-}$et $\mathrm{Ca}^{++}, \mathrm{SO}_{4}{ }^{-}$et silice dissoute. Nous voyons que les variations sont très fortes pour la silice de 0,03 à $3,64 \mathrm{mg} / 1$ pour une même station. Pour 1'ensemble du Léman on peut noter des variations par rapport à la moyenne des valeurs, de $27 \%$ pour les carbonates, $18 \%$ pour le Ca et $14 \%$ pour les sulfates.

En général les ions évoluent dans les mêmes sens, aussi les variations des concentrations globales sont-elles encore plus fortes comme le montre le tableau $\mathrm{III}_{4}$ 。
TABLEAU $\operatorname{III}_{4}$ : Valeurs extrêmes de la concentration globale, rencontrées dans le Léman

de Novembre 1967 à Septembre 1970

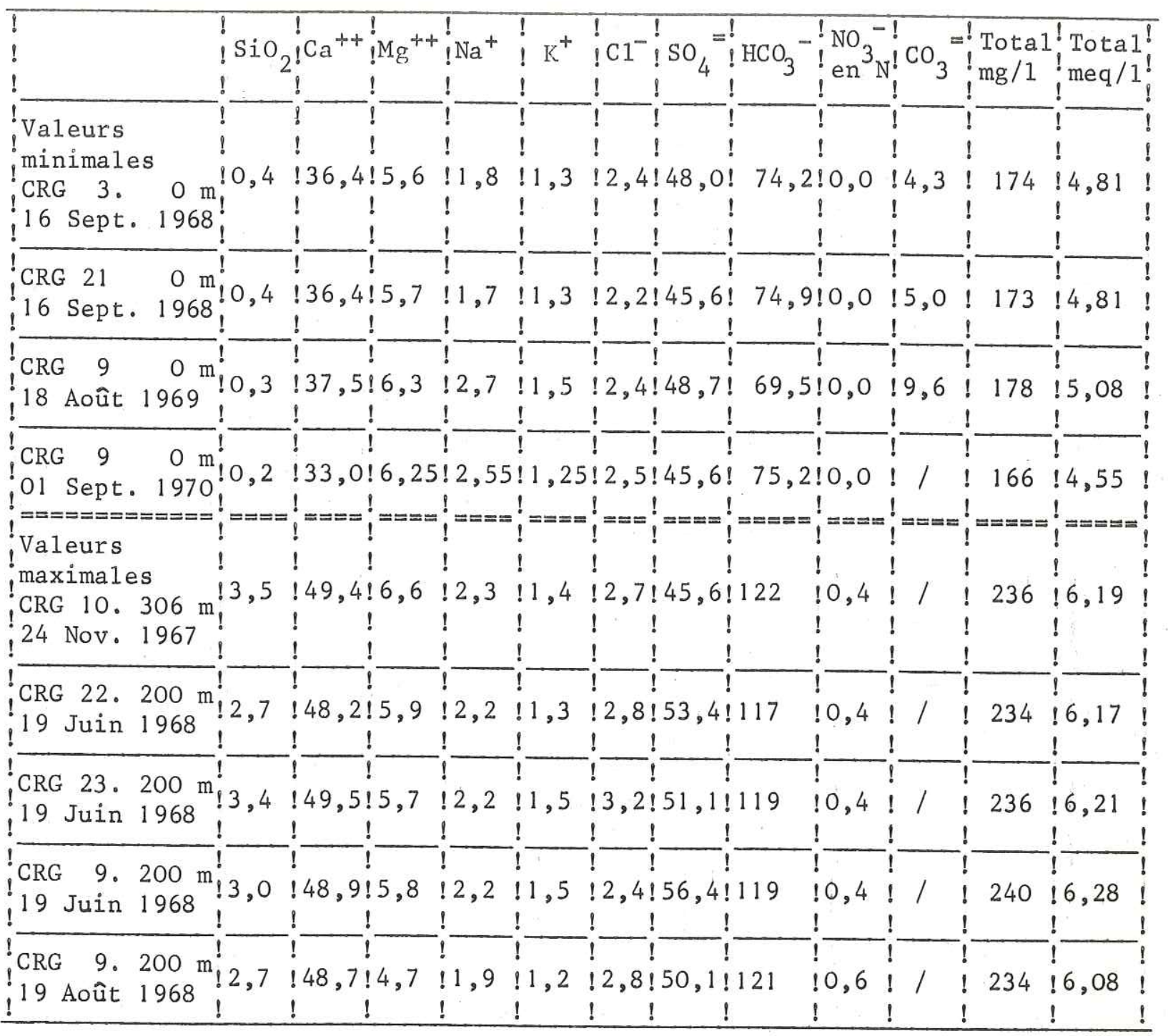

I1 est à remarquer qu'on devrait pouvoir trouver dans les eaux de fond au point CRG 10 des valeurs encore plus élevées (240 à $245 \mathrm{mg} / 1$ ) si une surveillance suivie était effectuée. Quoiqu'il en soit les êcarts sont encore plus grands que ceux observés pour chaque ion en particulier : on a des variations de $40 \%$ pour la concentration globale en $\mathrm{mg} / 1$ et de $30 \%$ pour la 
somme des miliféquivalents ( $\mathrm{CO}_{2}$ dissout inclus). Entre les opinions de Forel et de Delebecque îl semble donc déjà que le savant français était le plus proche de la vêríté. Regardons d'où proviennent ces fortes variations en examinant de plus près les phénomènes au point CRG 3 .

3.3.2. - Etude physico-chimique et chimique du lac au point $\underline{C R G 3}$.

3.3.2.1. - Caractères physico-chimiques généraux.

Nous avons reporté sur la figure $\operatorname{III}_{3}$ l'évolution sur deux ans aux profondeurs $0,10,50,100$ et $170 \mathrm{~m}$ (fond) de la température, du $\mathrm{pH}$ et de 1'oxygène dissout. Il est également porté la transparence des eaux de surface.

Températures. En hiver $1^{\prime}$ ensemble des eaux est à $5,5^{\circ} \mathrm{C}$. les eaux se réchauffent vers le mois d'avril, la thermocline devient de plus en plus profonde et s'estompe vers 50 à $60 \mathrm{~m}$ au moi de Décembre. En Janvier et Février l'homogénéité thermique est de nouveau réaîsée (voir également la figure $I_{4}$ ).

$\underline{p H}$. En hiver le pH est homogène en général à 7,3 - 7,4. Le pH va augmenter rëgulièrement en surface jusqu'à 8,2 au moîs de Septembre puis diminue assez rapidement. En profondeur l'évolution du phénomène est moins nette mais on note toutefoîs un affaiblissement du pH jusqu'à 7,2 au fond. (Le pH maximum mesuré en surface en trois ans a été de 8,8 au point CRG 10 en Septembre 1970).

$\mathrm{O}_{2}$ dissout. Les variations sont plus irrégulières que celles des deux paramètres précédents. En hiver les teneurs sont en général les mêmes en surface et au fond $(10 \mathrm{mg} / 1)$ mais ce n'est pas toujours le cas, notamment pour 1'hiver 1967-1968. Vers 1e mois d'Avril, date du début du réchauffement, de I'allongement des jours et du meilleur

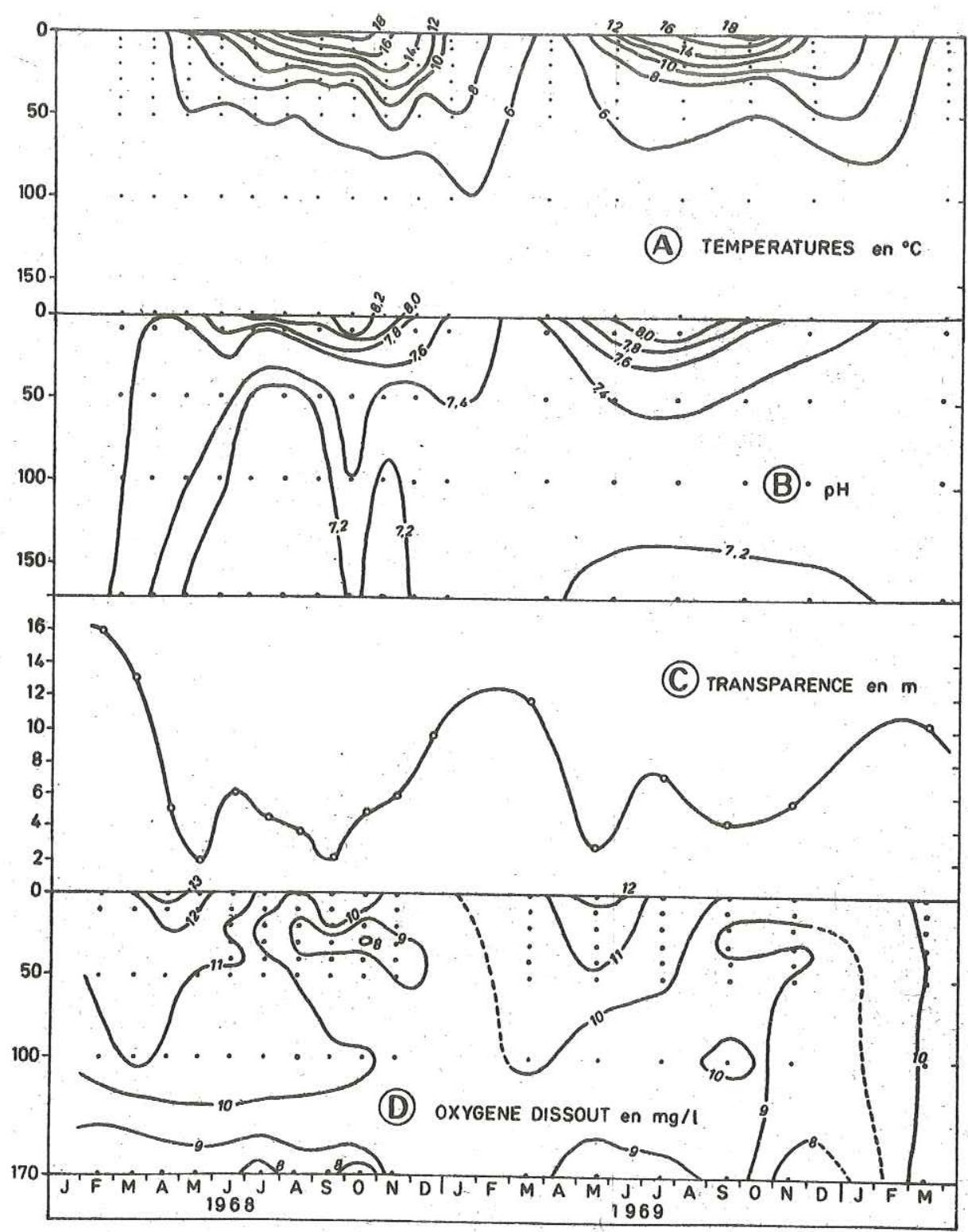

EMAN CRG 3 - Variations des paramètres tempórature, $\mathrm{PH}$ transparenco et $\mathrm{O}_{2}$ dissout de Fóvrier 1968 à Mars 1970 
ensoleillement un maximum est atteînt en surface (12 à $13 \mathrm{mg} / 1$ ). Les valeurs diminuent alors mais on observe à nouveau un maximum secondaire en Septembre. Nous n'entrerons pas dans le détail de ce mécanisme bien décrit par Monod (1956). Ce cycle est directement lié à celui du plancton dont le premier développement commence en Avril avec un maximum en Mai, la production d'oxygène par le plancton dépasse alors la déxoxygénation physique due au réchauffement des eaux. Après le mois de mai ce phênomène 1 'emporte, il est seulement retardé par le 2ème pic de développement du plancton au mois de Septembre. Dans les couches profondes les valeurs sont beaucoup plus constantes et sont toujours inférieures aux valeurs de surface ( 8 à $9 \mathrm{mg} / 1$ ). Ce phénomène est bien connu dans le Léman depuis les travaux de la Commission de Protection du Léman : le déséquilibre d'oxygène entre I'hypolimnion et I'épilimnion s'accrô̂t et la réserve d'oxygène des eaux profondes diminue chaque année. Le taux de saturation -dont nous n'avons volontairement pas parlé ici- a atteint $10 \%$ en $1969(1,05 \mathrm{mg} / 1$ d'oxygène). Nous reparlerons de ces phénomènes plus loin.

Transparence. Au point CRG 3, situé loin des zones d'apports terrigènes, la transparence reflète directement l'activité biologique du lac. En hiver à la production très faible de plancton correspond un maximum de transparence. Aux développements importants du plancton, en Mai et en Septembre, correspondent chaque fois un minimum de transparence.

\subsubsection{2. - Etude des éléments à fortes variations.}

Comme nous l'avons vu les bicarbonates, le calcium, les sulfates et la silice sont les éléments les plus variables pour l'ensemble du lac. Si on considère le point CRG 3 il en est de même ainsi que le montrent bien les figures $\operatorname{III}_{2}$ et $\operatorname{III}_{4}$.

Bicarbonates. Les équilibres des carbonates sont bien connus (Hutchinson). Pour le Léman la précipitation des carbonates s'effectue au mois de Septembre dans les couches superficielles de l'épilimnion quand les températures sont élevées $\left(>15,5^{\circ} \mathrm{C}\right)$ et les $\mathrm{pH}$ proches de 8,2. Cette précipitation est bien visible sur les diagrammes en 1968 et 1969. La teneur en $\mathrm{HCO}_{3}{ }^{-}$s'abaisse à $75 \mathrm{mg} / 1$. Parallèlement la teneur en acide carbonique $\left(\mathrm{CO}_{3}{ }^{-}{ }^{-}\right)$qui est nulle à toutes les profondeurs pendant une grande partìe de 1 'année passe en surface à $5 \mathrm{mg} / 1$ aux mois d'Août et Septembre. Une baisse beaucoup moins importante des bicarbonates est visible en Avril, au premier maximum du plancton, elle est accompagnée d'une faible apparition de carbonates (traces à 2 ou $3 \mathrm{mg} / 1$ ). Cette relation entre 1a précipitation des carbonates et 1'activité biologique est bien connue, elle est due à la consommation de $\mathrm{CO}_{2}$ dissout (il y en a dans le Léman jusqu'à $5 \mathrm{mg} / 1$, Monod 1956) par photosynthèse lors du développement du plancton. Remarquons que certains composants du plancton sont calcaires : Joukowisky et Buffle (1938) ont signalé la présence de coccolithophoridés.

A partir de $40 \mathrm{~m} \mathrm{le}$ cycle saisonnier des bicarbonates est presque nul, la principale variation est 1 'augmentation régulière en profondeur, les valeurs atteignent ici $115 \mathrm{mg} / 1$ à $170 \mathrm{~m}$. Cette augmentation est sans doute liée à une redissolution des carbonates en raison du pH plus acide $(7,2)$ et de la basse température $\left(5\right.$ à $6^{\circ} \mathrm{C}$ ). Remarquons que pour les zones peu profondes du lac cette redissolution n'a sans doute pas lieu, c'est une des raisons des fortes teneurs en $\mathrm{Ca} \mathrm{Co}_{3}$ des vases du petit lac, sur lesquelles nous reviendrons au chapitre IV.

Calcium. Les variations de cet élément sont analogues à celles des bicarbonates : les valeurs de surface passent de 45 à $46 \mathrm{mg} / 1$ en hiver, à $36 \mathrm{mg} / 1$ en Septembre. Il y a également un petit minimum secondaire en Avril. Cette diminution au mois de Septembre n'est sensible que dans l'épilimnion. En profondeur le Ca augmente régulièrement jusqu'à $48 \mathrm{mg} / 1$. Cette précipitation des carbonates en Septembre s'observe dans d'autres lacs voisins, notamment à Nantua (Vivier P, Orand A) 
Sulfates. La variation des sulfates est beaucoup moins régulière que celle des éléments précédents, néanmoins on observe toujours en surface des valeurs plus faibles qu'en profondeur; de plus il semble bien qu'il y ait un minimum en surface vers JuilletAoût. Ces phénomènes de surface avaient été notés par Monod (1956) mais les causes de ces variations sont encore mal connues, il est certain que le plancton joue un rôle, îl est possible que des bactéries interviennent également.

Silice. De tous les éléments que nous avons étudiés, la silice est celui qui est le plus variable (voir tableau III $_{3}$ ). Les caractères de la variation de la silice sont les suivants :

- fortes variations en surface (0-50 m) allant de plus d' $1 \mathrm{mg} / 1$ à $0,1 \mathrm{mg} / 1$.

- augmentation générale avec la profondeur à partír de $50 \mathrm{~m}$, avec souvent un taux d'augmentation accru vers $1 \mathrm{e}$ fond.

- variations saisonnières faibles en profondeur.

Les variations en surface sont étroitement liées à 1 'activité biologique : 1e minimum principal est atteint en étê (Juillet à Septembre), certaines espèces planctoniques comme les diatomées utilisant la silice dissoute. Mais il faut remarquer que le premier développement du plancton en Avril-Mai occasionne déjà une baisse sensible de la silice en surface. Un exemple type de la variation de la silice avec la profondeur est donné par l'analyse de Novembre 1967 au point CRG 10 (voir figure III $_{11}$ ).

Ce comportement de la silice s'observe dans les océans et de nombreux lacs (Hutchinson), notamment pour ceux proches du

Léman : lac du Bourget, d'Annecy et aussi de Nantua (Vivier, Serruya, Orand, 1967), où des valeurs semblables, de 3 à $4 \mathrm{mg} / 1$ au fond, sont observées malgré des profondeurs différentes. Nous nous proposons de revenir sur ces phénomènes dans des publications ultérieures. 
Nitrates. Nous ne développerons pas ici l'évolution des nitrates. Cet élément est si étroitement liế à la biologie que son étude nécessite une connaissance approfondie des conditions biologiques. Comme la silice, les nitrates varient fortement en surface : de $0,42 \mathrm{mg} \mathrm{N} / 1$ en Mars 1968 à $0,02 \mathrm{mg}$ M/1 en Septembre 1968 pour remonter à $0,37 \mathrm{mg} \mathrm{N} / 1$ en Décembre 1968. Au fond, si les valeurs sont toujours supérieures à celles de la surface $(0,59 \mathrm{mg} \mathrm{N} / 1$ en Juillet 1968) les variations saisonnières sont très atténuées.

D'autres éléments, mineurs, par rapport à la composition globale du lac, mais dont l'importance biologique est essentielle, peuvent présenter des variations importantes (nitrites, ammoniaque, phosphates). Notons aussi qu'en raison de la méthode peu précise de dosage -par différence- employée,l'étude détaillée du magnésium n'a pu être entreprise. Cet élément devrait se comporter comme le calcium mais ses variations ne semblent pas confirmer cette hypothèse : on n'observe pas du tout de diminution en Septembre. Nous verrons au chapitre IV la confirmation de cette différence de comportement.

\subsubsection{3. - Eléments à faibles variations.}

Un certain nombre d'éléments ne montrent aucune variation au cours de l'année, quelles que soient les profondeurs,il s'agit surtout du sodium, du potassium et des chlorures. Les seuls écarts observés sont à mettre au compte du hasard et de 1 'incertitude des mesures. Les incertitudes sur la dêtermination du magnésium nous l'avaient fait ranger dans cette catégorie. Cette constance a été vérifiée par la suite grâce au dosage direct par Absorption Atomique en 1970 le magnésium n'a presque pas variê même au mois de Septembre pour lequel le calcium a fortement baissé (voir tableau III $_{4}$ : CRG 9, O m, ler Septembre 1970)

3.3.2.4. - Conclusion de l'étude chimique du point CRG 3.

La première constatation est la différence entre la couche supérieure délimitée par la thermoclîne, c'est-à-dire de quelques mètres en Avri1 à $60 \mathrm{~m}$ en Décembre, et les couches profondes. Cette couche correspond à ce qu'on appelle généralement l'épilimnion dans les études de thermique, son extension verticale varie au cours de l'année.

Dans cette couche supérieure s'observent de très importantes variations saisonnières (figures $\mathrm{III}_{3}$ et $\mathrm{III}_{4}$ ) des caractères physico-chimiques. Les paramètres primaires comme la température, la lumière reçue et les sels nutritifs à disposition, vont conditionner la productivité du plancton, phénomène dont dépendent à leur tour les concentrations en bicarbonates, silice, nitrates, etc... Evidemment tous ces éléments interfèrent les uns avec les autres. Ainsi le développement du plancton va limiter la pénétration de la lumière et la teneur en silice en surface est sans doute un facteur limitant du plancton, comme semble l'attester les très faibles valeurs rencontrées au mois de Septembre. C'est pendant cette période, où l'épilimnion est le mieux individualisé, qu'ont lieu les changements chimiques les plus importants : précipitation des carbonates de calcium, diminution très forte de la silice et des nitrates due au deuxième développement annuel du plancton.

A côté des éléments variables il en est d'autres qui restent remarquablement constants, il s'agit du sodium, du potassium et des chlorures. Ces différences de comportement sont bien mises en évidence sur la figure $\operatorname{III}_{4}$ où nous avons reporté les valeurs moyennes dans la couche superficielle de tous les éléments dosés couramment au point CRG 3.

Enfin une minéralisation plus forte est notée pendant toute l'année en profondeur. Cette augmentation est très nette pour les bicarbonates et le calcium, encore plus pour la silice, mais légère pour les sulfates. En hiver les eaux superficielles sont entièrement homogènes, nous discutons plus loin ce phênomène. 


\subsection{3. - Etude chimique globale du Léman.}

Cette étude porte sur 1es points CRG 3, 4, 6, 9, 21, 22,23 . La composition du lac à la sortie de Genève sera étudiée à part.

\subsubsection{1. - Variations saisonnières}

\section{1) Concentrations élémentaires.}

Nous avons reporté dans le tableau $\operatorname{III}_{5}$ les valeurs moyennes pour la couche supérieure,des bicarbonates, du calcium, de la silice, du magnésium et des sulfates pour chaque point mois par mois.

Bicarbonates et calcium pour tous les points. Ces deux éléments se suivent étroitement pendant toute 1'année. En 1968, ce minimum de concentrations a lieu en Septembre pour 5 points, pour CRG 22 et CRG 23 ce minimum apparaît un mois plus tôt en Août. En 1969 cette évolution est analogue.

Sulfates. En 1968 la seule tendance générale est un minimum en Juillet, d'autres minimums secondaires se remarquent avec des décalages d'un ou deux mois suivant les points. Seuls les points CRG 22 et CRG 23 évoluent tout à fait parallèlement. La forte baisse du mois de Juillet s'observe de nouveau en 1969.

Silice. Pour tous les points les valeurs d'été (Juillet à Septembre) sont les plus faibles. On observe également un minimum secondaire marqué en Mai, à CRG 4 et CRG 9. En hiver les valeurs sont maximales partout.

Magnésium. Ainsi que nous l'avions dit aucune variation régulière n'est décelable.
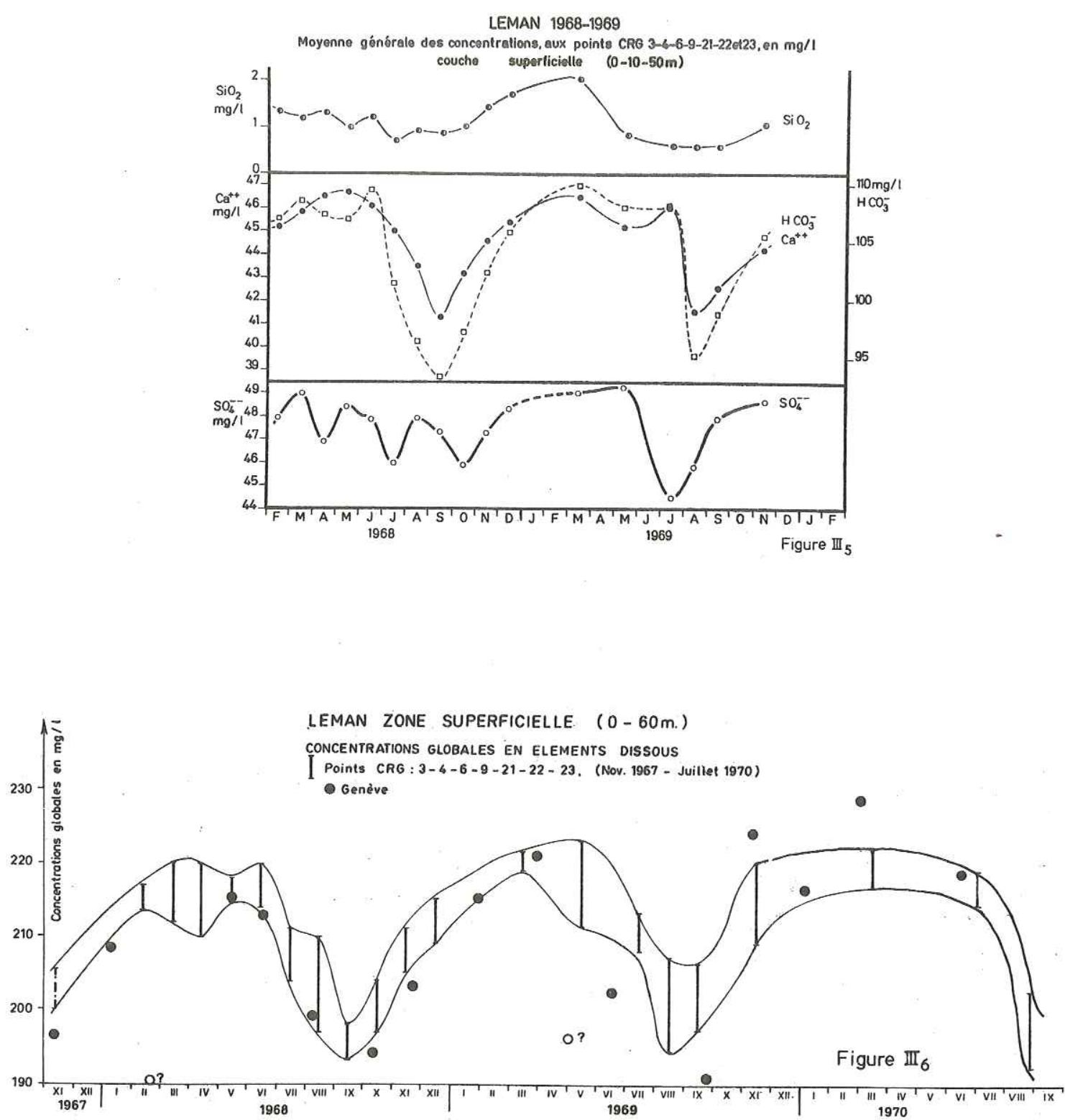
Sur la figure III $_{5}$ nous avons portê chaque mois les moyennes des teneurs de surface pour tout le Léman. La précipitation des carbonates qui se produit sur tout le lac à la fin de l'été est particulièrement visible. Pour la silîce, malgrê de légères irrégularités, le cycle général est bien vissible. Quant aux sulfates, comme nous 1 'avons dit, seule la baisse du mois de Juillet s'est reproduite de 1968 à 1969, mais nous avons manqué de mesures en 1969, les variations des sulfates étant assez brusques d'un mois à 1 'autre, il est nécessaire d'avoir au moins un échanti1lonnage mensuel. De plus la précision des analyses, plus faible par rapport aux autres éléments, limite les conclusions.

\subsubsection{1. - Variations saisonnières.}

\section{2) Concentrations globales.}

Nous avons porté sur la figure III $_{6}$ la variation mois par mois de la concentration globale moyenne du lac pour une couche donnée. Les barres verticales représentent la dispersion géographique des valeurs à un mois donné. Comme nous l'avions déjà dit les variations particulières des éléments s'ajoutent, la variation globale du lac est donc encore plus nette avec toujours un minimum à la fin de 1 'été.

\subsubsection{2. - Variation suivant la profondeur.}

\section{1) Concentrations élémentaires.}

Nous avons reporté dans les tableaux $\operatorname{III}_{6}$ et $\operatorname{III}_{7}$ en annexe les valeurs moyennes annuelles par couches et par éléments pour chaque point ${ }^{\S}$. En 1968 pour tous les points on note une variation très nette de la silice, des bicarbonates, du calcíum, des sulfates,

$\S$ Pour 1'année 1968, nous avons placé à part CRG 3 et CRG 4, en effet, 1e calcul des valeurs moyennes n'est pas rigoureusement le même du fait des profondeurs différentes.
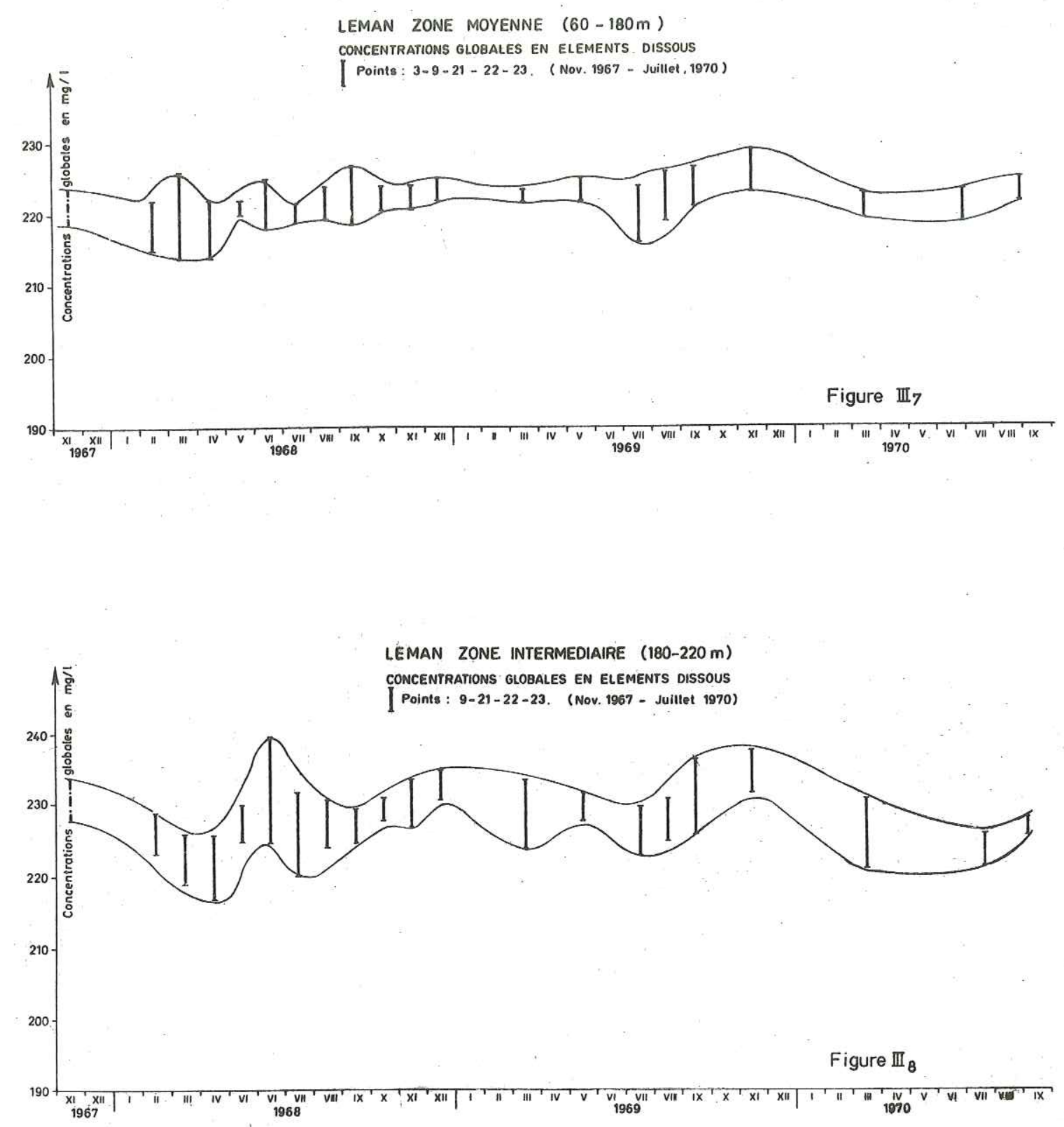
des nitrates. La variation est nulle pour le sodium, le potassium, le magnésium. Quant aux chlorures aucune tendance n'est décelable. Les mềmes constatations peuvent être faites en 1969.

I1 est très important de noter que les valeurs des sulfates et des nitrates sont sensiblement les mêmes pour les couches moyennes et intermédiaires : cela signifie qu'îl n'y a pas de changement au sein de 1'hypolimnion. Pour les bicarbonates, le calcium

et la silice, on note une augmentatîon constante avec la profondeur. Cette augmentation peut avoir deux origines : une diffusion à partir des sédiments gorgés d'eaux plus minéralisées ou bien une dissolution totale ou partielle des carbonates précipités et des squelettes silicieux du plancton mort. La deuxième hypothèse semble jusqu'icí la plus vraisemblable. Une étude des eaux intersticielles des vases est actuellement en cours avec $P$. Blanc.

\section{2) Concentration globale.}

Elle augmente donc régulièrement avec la profondeur, que1que soit 1'époque de 1 'année. Nous avons reporté dans le tableau III $_{8}$ en annexe les valeurs élémentaires moyennes par couches et par points sur deux ans. Les figures $\operatorname{III}_{6}$, III 7 et III $_{8}$ construites sur ces données illustrent bien le phénomène. En hiver la composition chímique du lac s'homogénéise beaucoup mais en général les eaux de fond sont toujours très légèrement plus chargées. Cette augmentation se remarque pour tous les points. Les barres verticales représentent la dispersion géographique des valeurs pour un mois donné.

\subsubsection{3. - Variations suivant les régions du lac.}

\section{1) Le Haut Lac. (Points CRG 22 et 23).}

La région de 1'embouchure du Rhône est caractêrisêe dans notre étude par les points CRG 22 et CRG 23 distants entre eux de $2,5 \mathrm{~km}$ et situés respectivement à $5 \mathrm{~km}$ et à $4 \mathrm{~km}$ de 1 'embouchure.
Les deux points sont aux mêmes profondeurs mais CRG 23 est situé dans le canyon du Rhône. Les évolutions et les concentrations sont les mềmes pour tous les éléments dissous à presque toutes les pro-

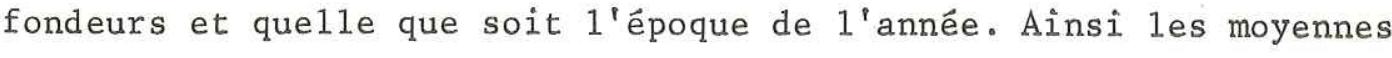
des éléments en 1968 et 1969 sont sensiblement les mềmes avec une légère exception pour les chlorures dont la variabilitê a déjà été remarquée (voir tableaux $\operatorname{III}_{6}$ et $\operatorname{III}_{7}$ ).

Par rapport à l'ensemble du lac les distinctions sont. très faibles : les plus fortes variations s'observent pour la silice $1,05 \mathrm{mg} / 1$, en surface dans le Haut Lac, contre $1,20 \mathrm{mg} / 1$ pour $1 \mathrm{e}$ reste du 1ac (1968), cette différence s'estompe presque totalement l'année suivante. Notons aussi que les eaux sont légèrement moins chlorurées dans 1e Haut Lac que dans 1e Grand Lac.

\section{2) Partie Centrale. (Points CRG 3, 4, 9, 21).}

Bien que ces points aient des situations assez différentes (profondeur, proximité des rives et des embouchures) leur comportement chimique est sensiblement le même. Les moyennes annuelles (tableaux $\operatorname{III}_{6}$ et $\mathrm{III}_{7}$ ) sont à peu près identiques pour toutes les couches, la seule exception étant pour la silice en surface, ce qui n'étonne pas vu le comportement à part de cet élément.

Du point de vue de l'évolution des éléments (tableau III $_{5}$ ) on note une bonne similitude entre CRG 3 et CRG 21, en particulier pour les sulfates (maximum en Mars 1968). I1 n'en est pas de même pour le point CRG 9 pour lequel on note un maximum important au mois de Juin 1968. Mais ces différences restent mineures par rapport à $1^{\prime}$ 'ensemble des éléments.

\section{3) Petit Lac. (CRG 6)}

Le point CRG 6 , situé à $60 \mathrm{~m}$ de profondeur dans le Petit Lac juste après le seuil d'Yvoire, est bien à part. En effet ses concen- 
trations moyennes sont presque toujours supérieures au reste du lac, pour la même couche superficielle (0-60 m), ainsi que le montrent les tableaux $\operatorname{III}_{6}$ et $\mathrm{III}_{7}$. En 1968 1a concentration moyenne de surface du Léman est de $208 \mathrm{mg} / 1$, celle de CRG 6 de $210 \mathrm{mg} / 1$ et en 1968 $209 \mathrm{mg} / 1$ contre $213 \mathrm{mg} / 1$. Il est vraisemblable que cette augmentation est due à une proportion non néglìgeable d'eaux des couches moyennes et mêmes profondes plus minéralisées qui remontent au seuil d'Yvoire. Ce phénomène est bien mis en évîdence sì on étudie les mouvements des masses d'eau par la géochimie isotopique (Hubert, Meybeck et Olive, 1970). Nous en reparlerons plus loin. L'évolution des divers éléments au point CRG 6 est assez analogue à celle de CRG 3 et CRG 21 .

\subsection{4. - Etude des eaux de L'émissaire à Genève.}

\subsubsection{1. - Comparaison avec le reste du lac.}

Nous avons effectué de Novembre 1967 à Mars 1970, 15 analyses complètes de $1^{\prime}$ eau du lac à sa sortie. Le point de prélèvement est situé à Genève à la jetée des Paquis sur 1a rive droite, à $750 \mathrm{~m}$ des vannes de sortie du lac. Dans 1'ensemble les eaux à Genève prêsentent les mêmes variations que les eaux de la couche superficielle du Léman, ainsi que le montrent les variations des concentrations globales (figure $\mathrm{III}_{6}$ ). Nous donnons les résultats complets des analyses sur le tableau $\operatorname{III}_{2}$ en annexe. Nous pensons que les très faibles valeurs du 19 Février 1968 et du ler Mai 1968, anormales pour cette époque de I'année sont sans doute dues à une précipitation des carbonates avant analyse. Si on fait abstraction de ces valeurs la variation des 3 éléments majeurs : bicarbonates, calcium et sulfates est rigoureusement identique entre CRG 6, à 1 'entrée du Petit Lac, et Genève. Notons entre autres la précipitation des carbonates en Septembre ainsi qu'un minimum secondaire de ces éléments en Avril-Mai. Pour les éléments mineurs, comme les nitrates ou les chlorures on observe des valeurs plus îrrégulières. Pour eux ìl serait nécessaire d'augmenter la fréquence des prélèvements (un prélèvement hebdomadaire).
Nous avons comparé les moyennes sur deux ans 1968-1969, de Genève (13 analyses) et de CRG 6 (16 analyses). Il s'agit ici de moyennes arithmétiques en $\mathrm{mg} / 1$.

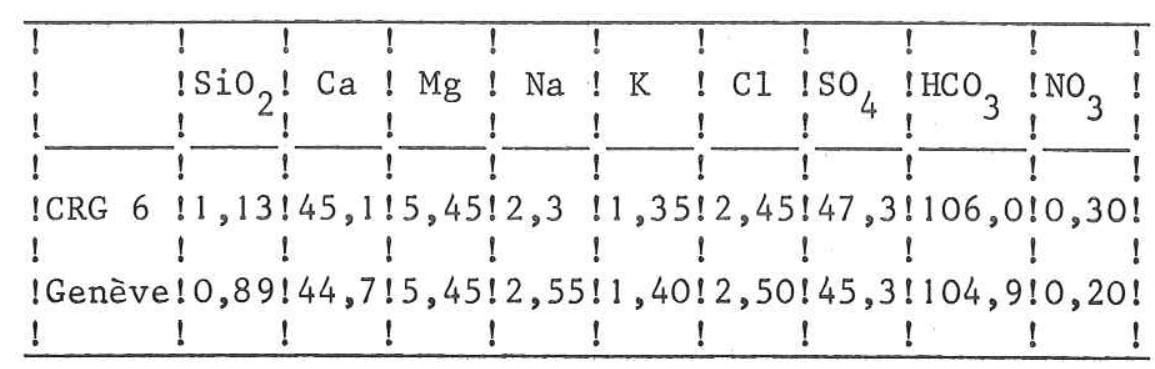

Les moyennes sont donc très semblables seules des différences s'observent pour la silice, les nitrates (mais le nombre de mesures n'est pas suffisant pour définir une moyenne), et dans une moindre mesure pour les bicarbonates et le calcium. L'influence des rivières qui se jettent dans le Petit Lac ne se fait pas beaucoup sentir. Cette similitude entre les deux points est expliquable : par rapport à l'ensemble du Léman le Petit Lac qui ne représente que 3,6 \% du volume global se comporte plutôt comme une rivière très large qui mettrait 4 mois à parcourir $20 \mathrm{~km}$. Les données isotopiques comparées entre CRG 6 et Genève confirment ce temps de transit que nous avions calculé en divisant le volume du Petit Lac par le volume mensuel de sortie à Genève. Les observations de Delebecque sont donc confirmées : 1'eau de 1 'exutoire a bien une composition chimique très proche des eaux de surface.

\subsubsection{2. - Teneurs moyennes à l'émissaire du Léman.}

Pour calculer la quantité d'éléments dissous passant à 1'émissaire, étant donné que la composition des eaux et les débits sont variables il faudrait chercher la relation entre les deux paramètres comme nous avons fait pour les apports, puis trouver une méthode de calcul pour pondérer les concentrations par les débits. 
Malheureusement il ne nous a pas été possîble d'effectuer cette recherche n'ayant que trop peu d'analyses. De plus vu 1'allure des variations chimiques et 1 a courbe des débits -1 'ouverture maximale des vannes est en été pour contrebalancer les apports du Rhône- il ne semble pas qu'il y ait de différences très nettes entre les moyennes arithmétiques et pondérées. La variabilité des eaux à Genève est celle de la couche superficìelle du lac, c'est-à-dire $30 \%$ par rapport à la moyenne, elle est très inférieure à celle des apports qui peut dépasser $100 \%$ suivant les débits. Nous avons pris pour calculer le bilan de sortie en 1968 les valeurs suivantes :

COMPOSITION MOYENNE DES EAUX A GENEVE EN 1968 EN MG/L

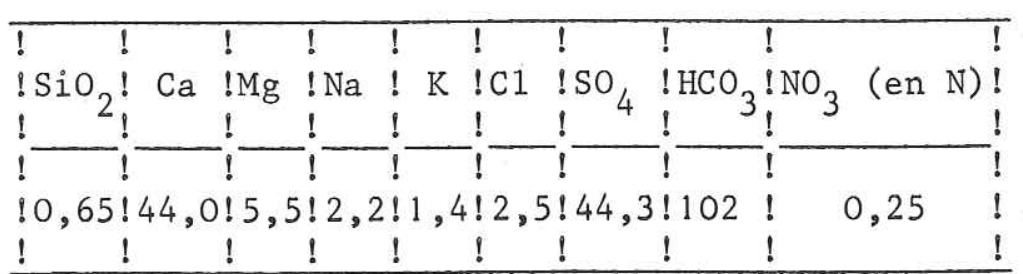

\subsection{5. - Conclusions de l'étude chimique globale du Léman.}

1) La composition des eaux est très peu différente suivant les régions du lac; les plus fortes variations moyennes annuelles n'exédent pas $3 \%$, par rapport à la moyenne, d'un point à un autre quant à la concentration globale. Elles sont du même ordre pour la plupart des ions sauf pour la silice où les variations régionales atteignent $30 \%$. Ces variations, maximales en surface, sont vraiment très faibles dans 1'hypolimnion où elles sont du même ordre de grandeur que les erreurs d'analyses.

2) La couche superficielle de 0 à $60 \mathrm{~m}$, qui correspond aux fortes variations thermiques et biologiques, est le siège d'importantes variations saisonnières essentiellement des bicarbonates, du calcium, des nitrates et de la silice. Par rapport aux moyennes mensuelles de la couche superficielle pour tout le lac, les variations dans cette couche sont de $15 \%$ pour les bicarbonates et le calcium, de 1 à 4 pour la silice qui est l'élément le plus variable dans le Léman, et de $12 \%$ pour les sulfates. En général les variations sont saisonnières sauf pour les sulfates qui évoluent beaucoup d'un mois à un autre.

3) Dans les couches moyennes et profondes, qui correspondent à 1'hypolimnion, à une profondeur donnée les variations saisonnières des éléments s'estompent beaucoup. Par contre on note une augmentation régulière de la silice, des bicarbonates et du calcium. Cette augmentation est de 1 à 10 et même parfoìs plus pour la silice, de $25 \%$ pour les bicarbonates et $18 \%$ pour le calcium. Pour les autres éléments 1'augmentation des teneurs au sein de 1'hypolimnion est très faible. catégories :

4) On peut alors classer les êléments étudiés en trois

\section{- Eléments à faibles variations.}

- Sodium et potassium : pas de variations sensibles.

- Chlorures : variations faibles surtout dépendantes de la région du lac.

- Magnésium : variations faibles.

\section{- Eléments à variations moyennes.}

- Sulfates : faibles variations, saisonnières et mensuelles, uniquement dans 1 'épilimnion sans doute liées au cycle biologique mais encore mal connues.

- Nitrates : variations saisonnières très nettes reliées au cycle planctonique dans 1'épilimnion, pas de variation nette en profondeur.

- Eléments à fortes variations.

- Bicarbonates et calcium : variations saisonnières fortes 


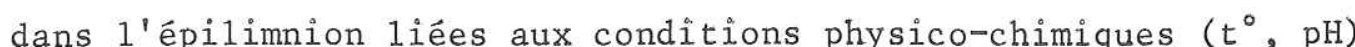
et biologiques ; minimum principal en Août-Septembre, minimum secondaire en Avril-Mai ; augmentation nette et régulière dans 1'hypolimnion jusqu'au fond. Elle peut être due à une redissolution partielle des carbonates précipitês en surface

- Silice : variations saisonnières très fortes dans l'épilimnion, directement liées au cycle biologique, minimum pendant tout l'été, maximum en hiver; augmentation nette et régulière dans 1 'hypolimnion jusqu'au fond, sans doute à cause d'une dissolution des squelettes siliceux du plancton (diatomées) mais une diffusion à partir des vases lacustres n'est pas exclue.

Les hypothèses que Delebecque avait formulées, en se basan sur 1'observation des phénomènes et sur quelques analyses, sont donc confirmées point par point.

3.3.6. - Concentration moyenne annuelle du Léman et variations interannuelles.

Pour comparer l'état chimique du Léman d'une année à l'autre il est nécessaîre de déterminer des concentrations moyennes annuelles. Nous avons vu que la variation avec la profondeur était la plus importante, nous avons donc basé notre méthode de calcul sur cette propriété. Nous avons divisé le lac en 4 couches pour lesquelles nous avons calculé la moyenne annuelle des teneurs. La couche superficielle est ainsi déterminée par 7 points, la couche moyenne par 5 points, et 1a couche intermédiaire de $180 \mathrm{~m}-220 \mathrm{~m}$ par 4 points. Cette dernière couche correspond plus à un artifice de calcul pour tenir compte des données à notre disposition, qu'à une réalité physique. Pour la couche profonde de $220 \mathrm{~m}$ à $310 \mathrm{~m}$ nous avons extrapolé les valeurs moyennes après avoir comparé les analyses sur CRG 10 effectuées deux ou trois fois dans 1 'année et les analyses régulières mensuelles. L'incertitude sur les données de cette couche profonde est donc plus grande que celle des autres couches. Les volumes partiels des couches étant bien connus (Hubault 1947) nous avons pu calculer les tonnages partiels pour chacune d'elles, puis le tonnage global du lac et enfin la concentration moyenne annuelle.

Les résultats pour les années 1968 et 1969 sont donnés dans les tableaux III $_{9}$ et III $_{10}$ en annexe. Dans $1^{\prime}$ ensemble il y a eu très peu de variations. Les bicarbonates ont augmenté en 1969 de $1 \mathrm{mg} / 1$, ce qui s'est reporté sur 1a concentration globale. Notons une diminution sensible des nitrates et une augmentation de la silice dans le même temps : ces variations peuvent se comprendre par des phénomènes biologiques et chimiques légèrement différents d'une année à l'autre, i1 se peut aussi que les apports des affluents aient une influence, mais elle doit rester faible vu l'énorme masse du Léman, nous en reparlerons à propos des bilans. Enfin la variation importante du sodium $2,63 \mathrm{mg} / 1$ en 1969 contre $1,99 \mathrm{mg} / 1$ en 1968 est tout à fait anormale vu le comportement de cet élément, elle est en effet à mettre au compte d'un changement de méthode d'analyse au début de 1969.

I1 nous semble intéressant de fournir également une analyse aussi complète que possible de l'eau du Léman. A côté de nos propres valeurs nous avons pris les valeurs moyennes des analyses très complètes publiées par Ramuz en 1957 ; pour les formes de l'azote et les phosphates les valeurs sont les moyennes pour tout le Lẻman en 1968 d'après les travaux de la Commission de Protection du Lểman. L'ensemble de 1 'analyse est sur $1 \mathrm{e}$ tableau $\operatorname{III}_{11}$, en annexe de ce chapitre.

\section{4. - Problème de la circulation générale du lac en hiver et du re- nouvellement des eaux.}

\subsection{1. - Position du problème.}

La circulation des eaux du Léman a fait 1 'objet de nombreuses études. Forel distinguait déjà les "courants d'écoulement du lac" dont la pénétration du Rhône est un aspect particulier, les 
"courants de convexion thermique" et les "courants causés par les vents". Mais la plupart de ces études concerne les courants superficiels qui sont les plus forts et les plus facilement étudiables, citons notamment la belle étude de Betant et Perrenoud (1932) sur les courants dans le Petìt Lac. Malgrê tout leur intérêt, ces études sont restées très localisées dans le temps et l'espace et aucune recherche d'ensemble sur le mélange des eaux n'avait été effectuée.

Seul Dussart (1948) avait abordé l'étude du devenir du Rhône et de 1a Dranse par les résistivités et les températures. Mais tous ces moyens d'étude étaient limités : seuils de détection trop élevés des courantomètres et homogénéité thermique, voire chimique du lac en hiver. Cette dernière particularité et le manque d'analyses chimiques complètes dans les couches les plus profondes avaient conduit la plupart des auteurs à 1 'hypothèse d'un retournement et d'un mélange complet des eaux de la surface jusqu'au fond à la fin de 1'hiver (Fore1, Delebecque, Monod 1956, etc...). Cette affirmation du mélange complet des eaux des lacs a deux origines :

- les lacs généralement étudiés à fond ont des profondeurs faibles, par exemple les lacs Windermere (Angleterre) et Mendota (U.S.A.). Le mélange des eaux peut donc se faire assez facilement par un apport d'énergie assez faible.

- 1es moyens d'étude sont en général basés sur des propriétés physiques de 1'eau : température, résistivité. Dès qu'il n'y a plus de variation verticale de ces paramètres on admet qu'il y a mélange.

\subsection{2. - Etude isotopique par le tritium.}

I1 suffit de trouver dans $1^{\prime}$ eau un élément qui ne présente pas les mêmes teneurs en surface et au fond pour affirmer que le mélange n'est pas complet. Le développement de la géochimie isotopique a permis de trouver pour les études hydrologiques un traceur particulièrement bien adapté puisqu'il fait partie de la molécule d'eau elle-même, il s'agit du tritium. Cet élément radioactif qui provient aujourd'hui, dans sa plus grande partie, des essais thermonucléaires se trouve dans tout le cycle de 1'eau, nous l'avons employé pour le Léman comme traceur et dateur naturel des eaux. Depuis 1964 le Centre de Recherches Géodynamiques de Thonon effectue des analyses de tritium trimestrielles à CRG 22, CRG 10 et CRG 6 ainsi que sur les rivières et 1'émissaire du lac. De nombreuses publications ont été faites (Blavoux et 01ive 1966 ; Meybeck, Hubert, Martin, Olive 1970 ; Hubert, Meybeck, 01ive 1970) ou sont en cours (thèses de MM P. Hubert et $\mathrm{Ph}$. Olive).

Comme ce n'est pas le propos de ce travail d'étudier la dynamique du lac nous nous limiterons aux conclusions générales concernant le mélange des eaux :

1) Il y a dans le Léman une stratification dynamique des eaux en trois couches:

- 1a couche profonde de $150 \mathrm{~m}$ à $300 \mathrm{~m}$ est caractérisée par des variations de teneurs en tritium lentes et très faibles. Ces eaux ont donc une grande inertie et se renouvellent très lentement.

- 1a couche moyenne de $50 \mathrm{~m}$ à $150 \mathrm{~m}$ présente des variations de teneurs importantes. Cette couche représente environ $45 \%$ des eaux du Léman et joue un rôle fondamental dans le renouvellement du $1 \mathrm{ac}$, on doit y voir le "courant d'écoulement du lac" de Forel.

- 1a couche supérieure : 1'action des vents, les variations thermiques et 1 'évaporation lui confèrent une dynamique particulière, bien révélée par les teneurs en tritium, irrégulières et très variables. Les mouvements des eaux y sont beaucoup plus violents que dans les deux autres couches et peuvent atteindre des valeurs supérieures à $10 \mathrm{~cm} / \mathrm{s}$. Les directions de ces courants sont également beaucoup plus aléatoires. 
$-84-$

w

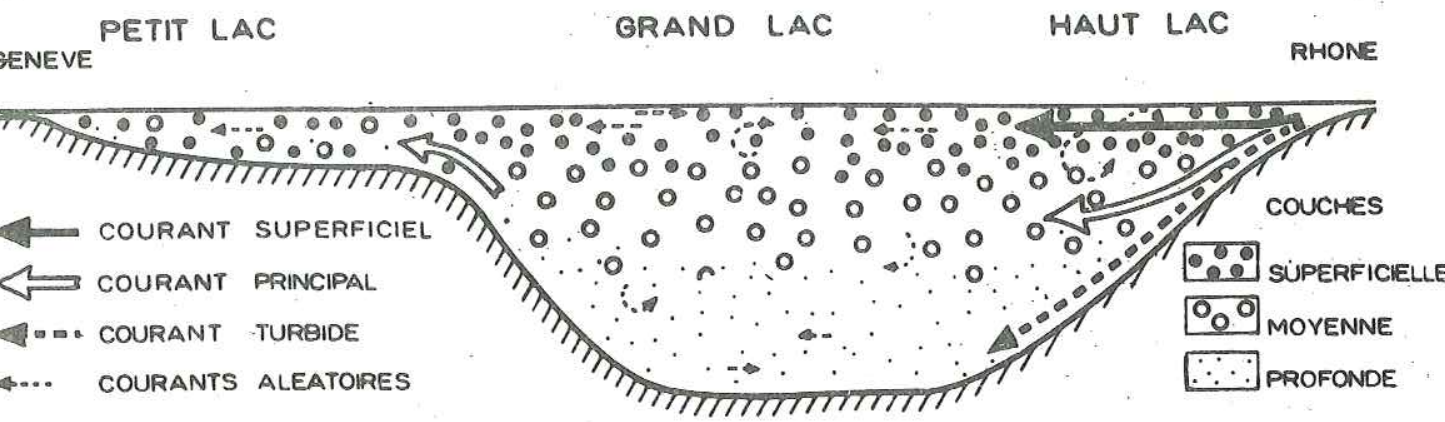

SCHEMA DE LA CIRCULATION DU LEMAN

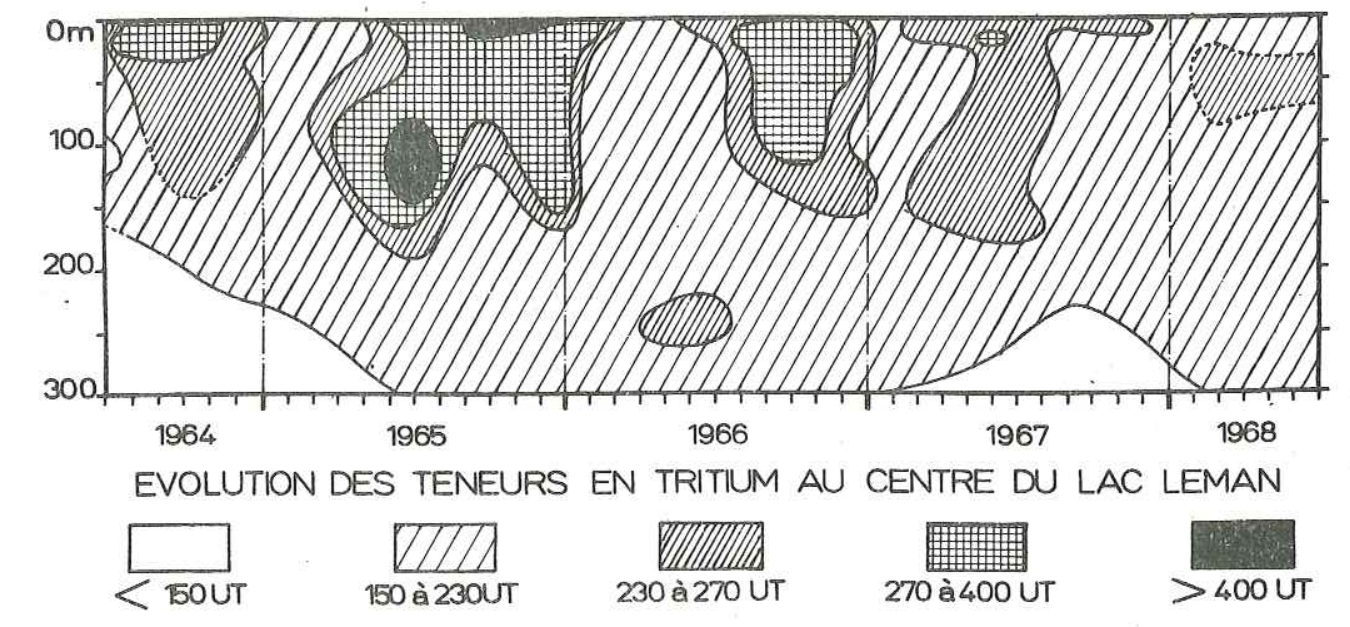

figure $\operatorname{III}_{10}$
Au seuil d'Yvoîre les données isotopiques montrent qu'il y a un mélange des 3 couches avec cependant une proportion majeure de la couche superficielle. Nous avons schématisé ces circulations sur la figure III $_{9}$.

2) En hiver seule la couche supérieure est fortement brassée, on a toujours une stratification isotopique mais elle est plus faible qu'en été. L'évolution des teneurs au point CRG 10 sur quatre ans met bien ce phénomène en évidence (figure $\operatorname{III}_{10}$ ).

\subsection{3. - Emploi de la silice comme élément traceur.}

Nous avons essayé de trouver un élément dissout qui puisse nous servir de traceur pour juger du mélange des eaux du lac. La rêsistivitê, employée par Dussart (1948), est une propriété globale de l'eau et ses variations peuvent être dues à de nombreuses causes différentes, de plus elles sont trop faibles pour faire des études précises. Il fallait un élément aux variations fortes et régulières surtout avec la profondeur, la perturbation des valeurs "normales" nous donnant alors des indications sur le mélange des eaux. La silice dissoute répondait bien à cette définition : à part dans la couche superficielle, où les variations saisonnières sont d'ailleurs bien définies, la silice augmente régulièrement vers le fond, en général à partir de $50 \mathrm{~m}$, profondeur à laquelle l'utilisation biologique de la silice est très faible. La courbe des teneurs au point CRG 10 en Novembre 1967 est typique de ce phénomène (figure $\operatorname{III}_{11}$ ).

En hiver les couches supérieures se mélangent et la teneur en silice s'homogénéise. En Février 1968 cette homogénéité se remarque jusqu'à $150 \mathrm{~m}$, de même en Mars 1969. En Mars 1970, nous observons pour la première fois une teneur identique de 1a surface au fond. Il semble donc qu'on ait là un mélange complet. Il nous faut noter la bonne concordance entre 1 a teneur en silice moyenne de Mars 1970 à CRG 10 : $1,80 \mathrm{mg} / 1$, avec les teneurs moyennes annuelles calculées suivant 

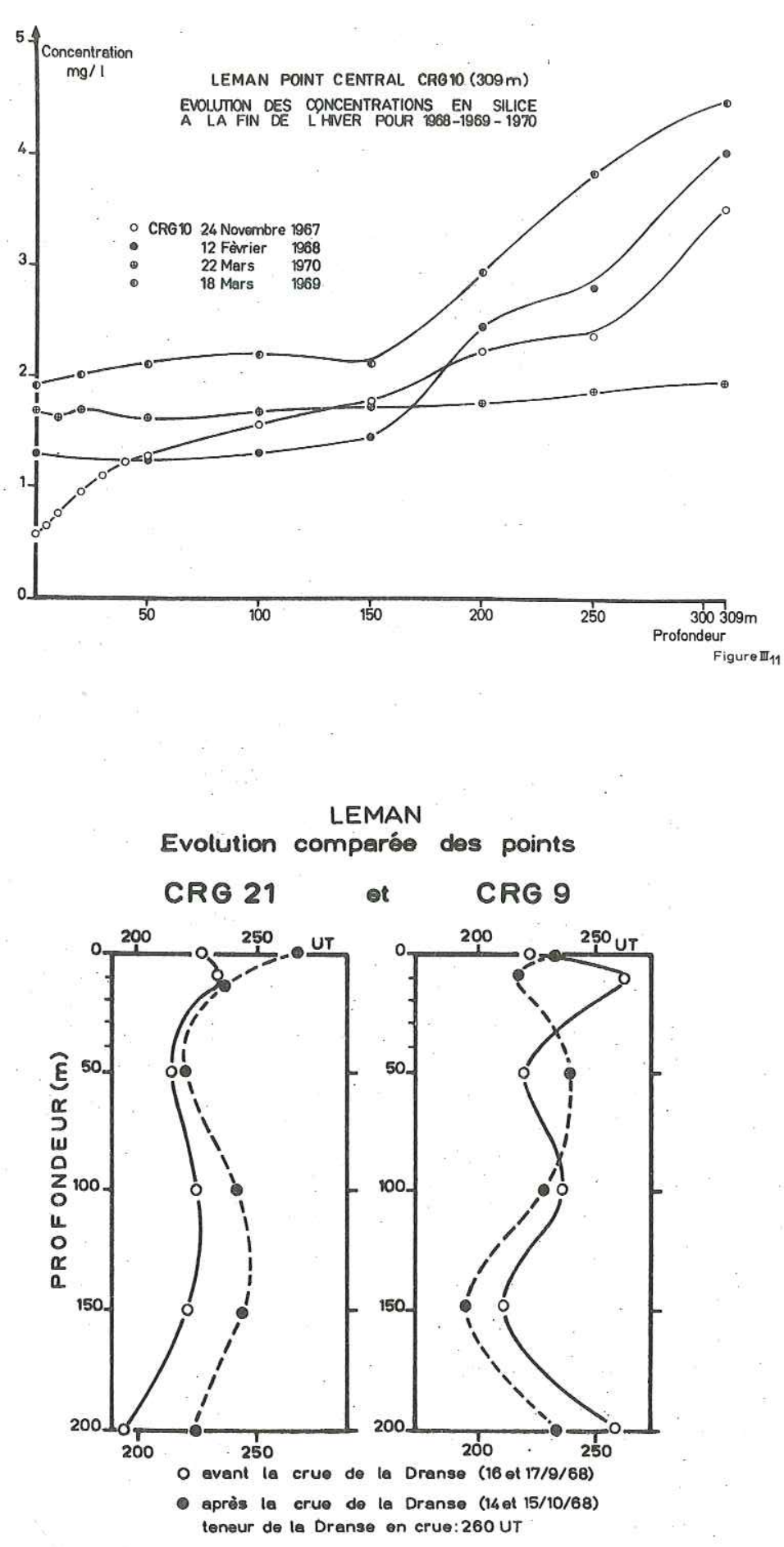

figure $\operatorname{III}_{12}$
On peut essayer de comparer ces résultats avec ceux obtenus par le tritium en 1968 : 1a figure III $_{10}$ montre encore un léger noyau de teneurs supérieures à 230 UT mais cette distinction est faible. En effet depuis 1965 date à laquelle la différence de teneurs en tritium entre les couches est maximum, il y a une uniformisation due à la diffusion, aux mélanges à la limite des couches, et secondairement à la décroissance radioactive du tritium, l'impulsion des essais thermonucléaires de 1962-1963 s'estompe de plus en plus. La comparaison des deux méthodes n'est donc pas concluante. Néanmoins i1 est certain qu'en 1968 et 1969 les eaux profondes n'ont pas été mélangées.

\subsection{4. - Conclusion.}

Il semble que la vérité se trouve entre un mélange complet des eaux et nos premières conclusions tirées d'observations isotopiques de 1964 à 1968 : le mélange des eaux est variable, en gênéral il peut atteindre 100 à $150 \mathrm{~m}$ de profondeur. D'après les teneurs en silice en 1970, il est possible, mais non encore prouvé, qu'il puisse atteindre $1 \mathrm{e}$ fond du lac. Ce phénomène a lieu à la fin de l'hiver, en Février-Mars, période où l'instabilité du lac est la plus grande du fait de 1 'homogénéité thermique.

\section{5. - Pénétration du Rhône et de la Dranse dans le Léman.}

\subsection{1. - Etudes antérieures.}

Forel rapporte (Le Lêman, tome $I, p .387$ ) que les auteurs anciens comme Strabon, Pomponius Mela ou Ammien Marcellin, affirmaient que "le Rhône traversait le Léman sans se mêler aucunement aux eaux du lac". Cette croyance encore tenace chez beaucoup de riverains du lac provient sans doute du phénomène connu sous le nom de Batallière : les eaux du Rhône chargées de suspensions sombrent très rapidement à 
$100 \mathrm{~m}$ ou $200 \mathrm{~m}$ de 1 'embouchure dans le lac et la différence entre ces deux types d'eaux est très tranchée. Pour le savant vaudois "les eaux descendent directement et pendant la plus grande partie de l'année, en suivant le ravin sous lacustre du delta du Rhône, dans les grands fonds du lac et s'y étalent en nappe horizontale". Forel se base pour cela essentiellement sur des considérations théoriques de densités et aussì sur des arguments sédimentologiques. Pour Delebecque 1'action des affluents ne se fait sentir qu'à une distance très faíble de l'embouchure. Dussart (1948) étudie en détail la pénétration du Rhône par la mesure des résistivités à des profondeurs variées en s'éloignant de plus en plus de cette rivière. $L^{\prime}$ étude est menée sur un cycle hydrologique complet. Dussart arrive aux conclusions suivantes :

- En hiver : 1e Rhône, plus froid que le lac et aux eaux plus minéralisées, s'enfonce et creuse lentement son ravin sous-lacustre. Les eaux se trouvent bien canalisées au début mais un peu plus loin se forme une "nappe secondaire" entre la surface et le fond.

- En été : 1e Rhône est beaucoup plus froid que les eaux superficielles, ses eaux moins minéralisées sont riches en suspensions. La position d'équilibre du Rhône est alors située vers 15-20 mètres. On peut déceler cette influence jusqu'à Thonon.

La méthode sur laquelle s'appuie Dussart nous semble très délicate si on effectue des interprétations sans tenir compte des variations du chimisme de l'eau. Ces variations sont maximales en été et dans les couches supérieures, or c'est dans ce domaine que Dussart a particulièrement travaillé. Pour cet auteur toutes les variations de résistivité observées sont à mettre au compte de 1'influence du Rhône. Or nous avons vu que la diminution des bicarbonates, qui entraine la plus grosse variation de la résistivité, a lieu sur le lac partout au même moment.
S'il semble bien qu'une partie du Rhône pénètre dans la couche superficielle en été (Meybeck et a1, 1970), nous verrons par contre que le canyon du Rhône est creusé par des courants turbides violents lors des crues, c'est-à-dire principalement en été.

3.5.2. - Etude chimique générale de la pénétration de la Dranse et du Rhône.

Notre étude des points CRG 22 et CRG 23 nous a montré qu'en ce qui concerne les valeurs moyennes annuelles les différences avec le reste du lac étaient très faibles pour 1 'ensemble des éléments dissous. La silice seule est légèrement plus faible dans le Haut Lac. Dans les grandes lignes les variations de teneurs au long de l'année sont identiques à celles du reste du lac, seule la variation des sulfates est différente mais nous avons également noté des différences entre les points CRG 9 et CRG 21. En ce qui concerne les concentrations des eaux de fond on devrait s'attendre à des différences notables entre les eaux du canyon (CRG 23) et les eaux de 1a levée nord (CRG 22). Or, si on compare les valeurs $\mathrm{Ca}^{++}, \mathrm{Mg}^{++}$, $\mathrm{HCO}_{3}{ }^{-}, \mathrm{SO}_{4}{ }^{-}$et silice reportées dans le tableau III $_{5}$, on voit que les teneurs comme le sens des variations sont identiques, il est donc difficile de déterminer par la chimie une différence de comportement entre ces deux points. Il en est de même pour le point CRG 21 . I1 faut donc faire appel à de nouvelles méthodes. Là encore nous avons utilisé le tritium, à la fois pour tracer les eaux et pour les dater. Cette étude a déjà fait l'objet d'une publication (Meybeck, Hubert, Martin, 01ive 1970), d'autres travaux complémentaires sont en cours ( $P$. Hubert).

\subsection{3. - Etude isotopique par le tritium.}

Sans entrer dans le détail, citons seulement deux exemples typiques de 1'emploi du tritium : 


\section{Pénétration de la crue de la Dranse du 22 Septembre 1968.}

Cette très forte crue dont nous avons déjà parlé dans le chapitre II a amené au Léman 62 millions de $\mathrm{m}^{3}$ d'eau en quelques jours (Hubert et al 1969). Il était intếressant de voir où sont allées ces eaux. En surface du lac, de 0 à $20 \mathrm{~m}$, un courant turbide était très visîble jusqu'à Thonon, mais les eaux de la Dranse ne semblaient pas du tout se diriger vers le nord. Nous avons étudié l'évolution avant et après 1 a crue des points CRG 21 , à $1 \mathrm{~km}$ de 1 'embouchure de 1a Dranse, et CRG 9 situé à $11 \mathrm{~km}$ de là près des côtes suisses. Nous avons ainsi pu mettre en évidence par le tritium deux autres courants dirigés vers le nord. En effet à la station CRG 21 1a teneur avait augmenté en surface et dans une zone située autour de $100 \mathrm{~m}$ de profondeur alors qu'à la station CRG 9, de situation géographique et de profondeur analogues à celles de CRG 21 l'évolution était inverse (figure $\operatorname{III}_{12}$ ). Cette variation au point CRG 21 est donc due à 1'influence de la Dranse dont la teneur (260 UT) était supérieure à celle du 1ac. Notons que les compositions chimiques avant et après 1a crue étaient identiques. Seuls les bicarbonates ont augmenté au fond $120 \mathrm{mg} / 1$ en Octobre contre $115 \mathrm{mg} / 1$ que1ques jours avant la crue, cette augmentation qui ne concorde pas avec une augmentation générale du lac à la même profondeur, pourrait être due aux eaux de la Dranse ( $160 \mathrm{mg} / 1$ lors de 1a crue).

L'étude comparée de CRG 21 et de la Dranse ainsi qu'une étude complète en hiver nous ont amené à la conclusion suivante :

- 1a pénétration des eaux de la Dranse dans le lac s'effectue suivant deux zones privilégiées : une zone superficielle et une zone de profondeur moyenne. Ces deux masses d'eau, d'origine fluviale récente, restent séparées par un coin "d'eaux lacustres" plus anciennes.

\section{Pénétration du Rhône et courants turbides.}

Des campagnes d'hiver et d'été nous conduisent à penser que pendant la crue annuelle du Rhône, les eaux s'écoulent principalement suivant 1'axe du canyon, à la foìs en surface et dans une zone moyenne vers $100 \mathrm{~m}$ de profondeur. En plus de ce phénomène permanent durant tout l'été nous avons pu mettre en évidence et étudier les courants turbides qui ont lieu dans le canyon du Rhône.

Nous avons déjà vu que la morphologie sous-lacustre du HautLac est caractêrisêe par 1'existence d'un canyon dont la tête est située à l'embouchure du Rhône, et qui aboutit $15 \mathrm{~km}$ plus 1 oin à la grande plaine centrale d'une profondeur de $309 \mathrm{~m}$. Ce canyon joue un grand rôle dans le comblement du Léman en canalisant les apports dêtritiques du Rhône : ce phênomène est analogue aux courants de turbidité bien connus dans les océans. Nous reviendrons sur l'importance sédimentologique de ces phénomènes au chapitre suivant.

Les prélèvements ont été effectués en utilisant des boutei1les hydrologiques classiques et un carottier Jenkins - Mortimer ${ }^{\S}$ qui ramène une petite carotte mixte eau - sédiment superficiel $(\mathrm{L}=60 \mathrm{~cm}$, $\phi=10 \mathrm{~cm})$. La surface du sédiment n'est presque pas perturbée lors du prélèvement. Cette étude a été réalisée en Juin 1968. En effet c'est à cette période située au début de 1a crue du Rhône qu'on a le plus de chance d'observer des courants turbides. Les prélèvements sont localisés sur la figure $\mathrm{IV}_{11}$

Deux courants turbides ont pu être étudiés le 19 et $1 \mathrm{e} 26$ Juin 1968 : les eaux prises par carottages dans le canyon étaient très chargées, de 1'ordre de plusieurs grammes par litre alors que la teneur en suspensions du lac est normalement mille fois plus faible.

$\S$ Cet appareil nous a été prêté par la Station d'Hydrobiologie Lacustre de I'I.N.R.A. que nous avons plaisir à remercier ici. 
Ces fortes turbidités sont significatives et ce phénomène a été observé en plusieurs points différents du canyon : par $195 \mathrm{~m}$ (carotte LM 7) et 122 m (LM 8) 1e 19 Juin 1968, et par 150 m (LM 9) 1e

26 Juin. Aucun prélèvement effectué à la bouteille à plus de $3 \mathrm{~m}$ audessus du canyon n'a étế turbìde, ce qui localise bien le courant. Les carottes prises aux mêmes dates sur les flancs du canyon (LM 6) et dans la plaine centrale à CRG 10 (LM 4) étaient particulièrement limpides. Nous avons complété l'étude en prêlevant dans toute la colonne d'eau au-dessus des carottes et dans le Rhône.

Le 19 Juin 1968 les eaux de fond dans le Haut Lac ont une teneur de 140 UT. Les eaux prélevées dans le courant turbide ont des teneurs très différentes et beaucoup plus fortes : 207 UT pour $1 \mathrm{e}$ prélèvement le plus proche du Rhône (LM 8) et 189 UT pour le plus éloigné (LM 7). Le Rhône ayant à ce moment là une teneur de 220 UT, on peut done attribuer aux eaux de ce courant une origine fluviale très récente. Le courant est également bien localisé dans le canyon. En effet on remarque qu'au point CRG 10, situé loìn dans 1a plaine centrale, les valeurs observées pour la carotte LM 4 (177 UT) sont bien les mêmes que pour les eaux de fond (177 UT à $250 \mathrm{~m}$ ). Le 26 Juin 1968 la teneur de la carotte turbide LM 9 (194 UT) est également analogue à celle du Rhône. Remarquons que les deux courants observés ont eu lìeu lors de la montée des eaux pendant une crue, par contre le 17 Juillet en période de décrue rapide, aucun des carottages effectués dans le canyon n'avait été turbide.

En conclusion les courants turbides du canyon du Rhône sont relativement rapides et très localisés dans le fond du canyon. Ils ont lieu essentiellement pendant les crues, se remarquent nettement jusqu'à $4 \mathrm{~km}$ de $I^{\prime}$ embouchure du Rhône et peuvent parcourir tout le canyon.

S'il n'avait pas été possible de mettre en évidence la pénétration profonde de 1a crue de 1a Dranse par les analyses chimiques -même par la silice- îl n'en est pas de même pour les courants turbides. En effet les analyses de tritium nous mermettent d'attribuer aux eaux du Rhône plus de la moitié des eaux du couran du 19 Juin 1968. Les analyses complètes du Rhône, de l'eau du courant turbide et de $1^{\prime}$ 'eau limpide à $3 \mathrm{~m}$ au-dessus du fond au point CRG 23, montrent cette origine (tableau $\operatorname{III}_{12}$ ).

\section{TABLEAU III 12 : ANALYSE CHIMIQUE D'UN COURANT TURBIDE (en mg/1)}

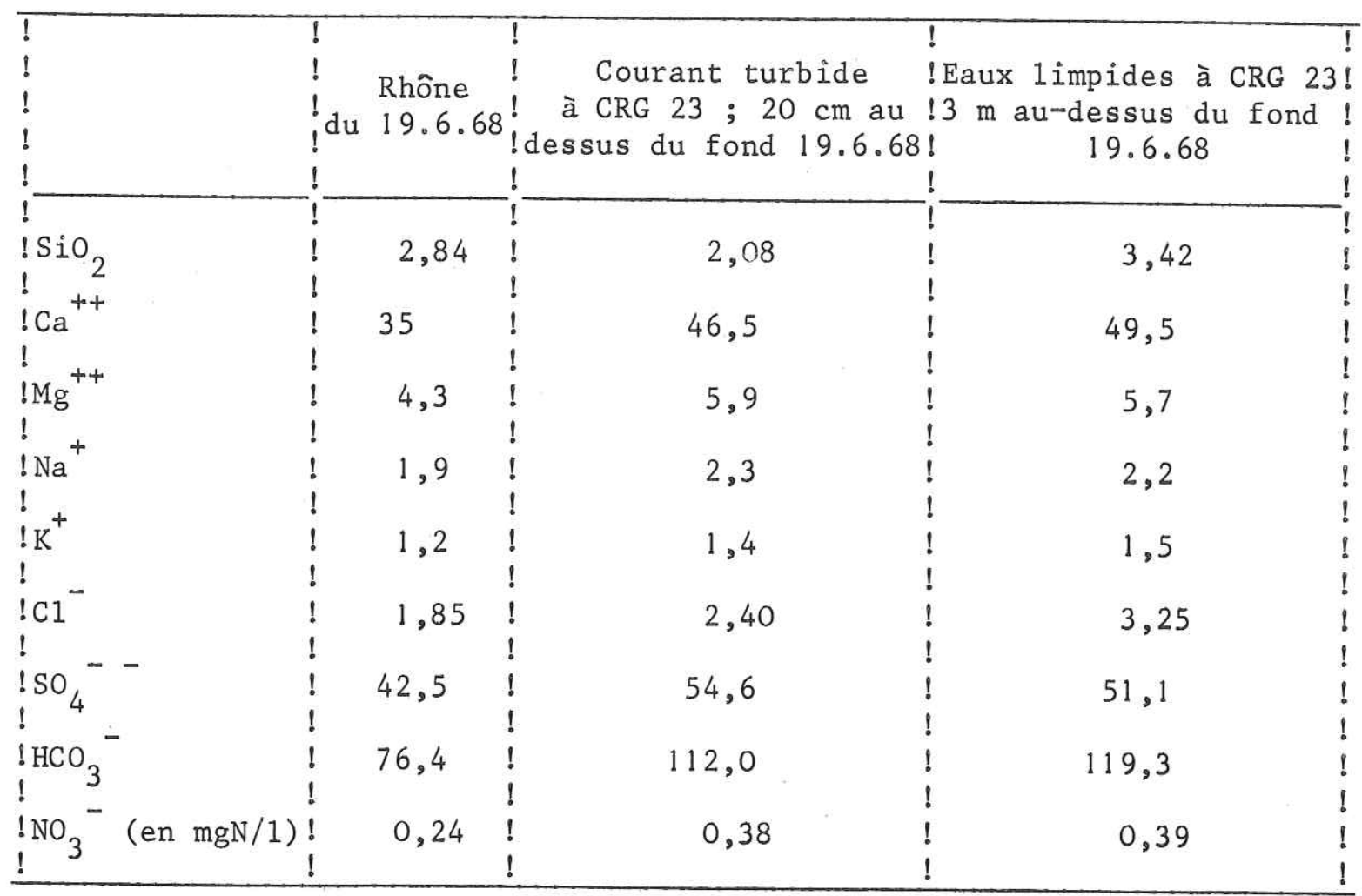

Pour la plupart des éléments majeurs -exception faite des sulfates- la composition du courant turbide se rapproche beaucoup de celle du Rhồne ; la valeur de la silice, très faible à cette profondeur, est particulièrement significative.

Remarque.

1) On peut maintenant expliquer la similitude entre le chimisme 
des eaux de fond de CRG 22 et de CRG 23 ; en effet nous prélevons toujours pour les analyses chimiques à 2 ou $3 \mathrm{~m}$ au-dessus du fond afin que le prélèvement ne soît pas pollué par la vase mise en suspension après 1 'arrivée de la bouteîlle (le poids de garde ne suffit pas toujours à arrêter la bouteille avant qu'elle ne touche). Aussi comme les courants turbides sont très localisés et qu'ils n'ont pas d'influence chimique sur les eaux immédiatement sus-jacentes, aucune différence n'était évidemment décelable.

2) L'influence chimique de ces eaux turbides dans la couche profonde du Léman doit rester très faible : ces courants sont localisés dans le temps et l'espace. Enfin si les eaux sont encore bien individualisées sur le plan chimique à $4 \mathrm{~km}$ du Rhône, on doit s'attendre à un mélange beaucoup plus fort au débouché du canyon dans la plaine centrale.

\section{6. - Evolution des caractères physico-chimiques du Léman depuis 1870 \\ 3.6.1. - Evolution des éléments principaux.}

C'est encore à Forel que nous devons de précieuses recherches historiques sur les anciennes analyses chimiques du Léman : la première fut celle de Tingry en 1808 et fut publiée en "pouces du pied de roi et en grains de la livre poids de marc", Forel heureusement transcrivit cette analyse dans le système métrique. Les analyses suivantes furent celles de St Claire Deville (1848) Risler Walter (1872) et Lossier (1877). Avec Duparc (1888), Freundler (1891) et Delebecque (1896), le lac est analysé presque couramment et les premières analyses complètes sont effectuées. De son côtê Forel multiplie les observations sur le lac notamment les mesures de transparence.

Après 1900, les analyses de Dienert (1912) -effectuées en vue d'alimenter Paris avec les eaux du Léman- de Monod (1956) et de Ramuz (1957) sont les plus marquantes. Il en résulte que nous pouvons retracer l'évolution chỉmique du Léman depuis plus d'un siècle. Nous avons porté sur la figure $\operatorname{III}_{13}$ la variation des concentrations depuis $\mathrm{l}^{\prime}$ analyse de Tingry pour les êlêments suivants : $\mathrm{Ca}^{++}{ }^{++} \mathrm{Mg}^{++}{ }^{++}{ }^{+}, \mathrm{K}^{+}$ $\mathrm{Cl}^{-}{ }^{-}$et $\mathrm{SO}_{4}{ }^{-}{ }^{-}$. Malgrê les dispersions -comme pour le $\mathrm{Mg}$ - dues aux différentes méthodes d'analyses, on note dans l'ensemble une remarquable constance de la composition chimique. Seuls les chlorures ont augmentê nettement de $1 \mathrm{mg} / 1$ en 1850 à $2,5 \mathrm{mg} / 1$ en 1970. Les autres éléments sont constants $\left(\mathrm{Na}^{+}, \mathrm{K}^{+}, \mathrm{Mg}^{++}\right.$) ou en très légère augmentation $\left(\mathrm{Ca}_{9}^{++} \mathrm{SO}_{4}{ }^{-}\right)$, ce qui peut être dû aussi au nombre plus grand d'analyses d'eaux profondes plus minéralìsées.

\subsection{2. - Evolution du caractère eutrophe du Léman.}

\subsubsection{1. - Transparence.}

La transparence est une mesure simple qui apporte beaucoup de renseignements sur 1'état d'un lac pour autant qu'on effectue un grand nombre de mesures. Pour le Léman ce facteur dépend essentie1lement du dêveloppement du plancton. Si la masse planctonique s'accrô̂t d'année en année, phénomène d'eutrophisation, la transparence tendra à diminuer. C'est exactement le cas du Léman : en 1874 Forel avait 10,2 $\mathrm{m}$ de transparence moyenne annue1le (241 observations sur 6 points), en 1968 cette valeur, calculée par la Commission de Protection du Lẻman, est passée à $6,9 \mathrm{~m}$.

De plus l'allure générale de la variation annuelle a radicalement changé (figure $\operatorname{III}_{14}$ ). Du temps de Forel il n'y avait qu'un minimum annuel de transparence en Juillet, maintenant on note chaque année deux minima en Mai et Septembre qui correspondent aux deux dêveloppements du plancton. C'est un signe frappant des changements biologiques de 1'état du lac. Enfin remarquons que le lac arrive encore à résorber cette énorme masse de plancton puisque la transparence en hiver atteint encore celle de 1874. C'est en étê que la situation se dégrade et des transparences moyennes inférieures à $2 \mathrm{~m}$ ne sont plus rares dans le Léman. 
$-96-$
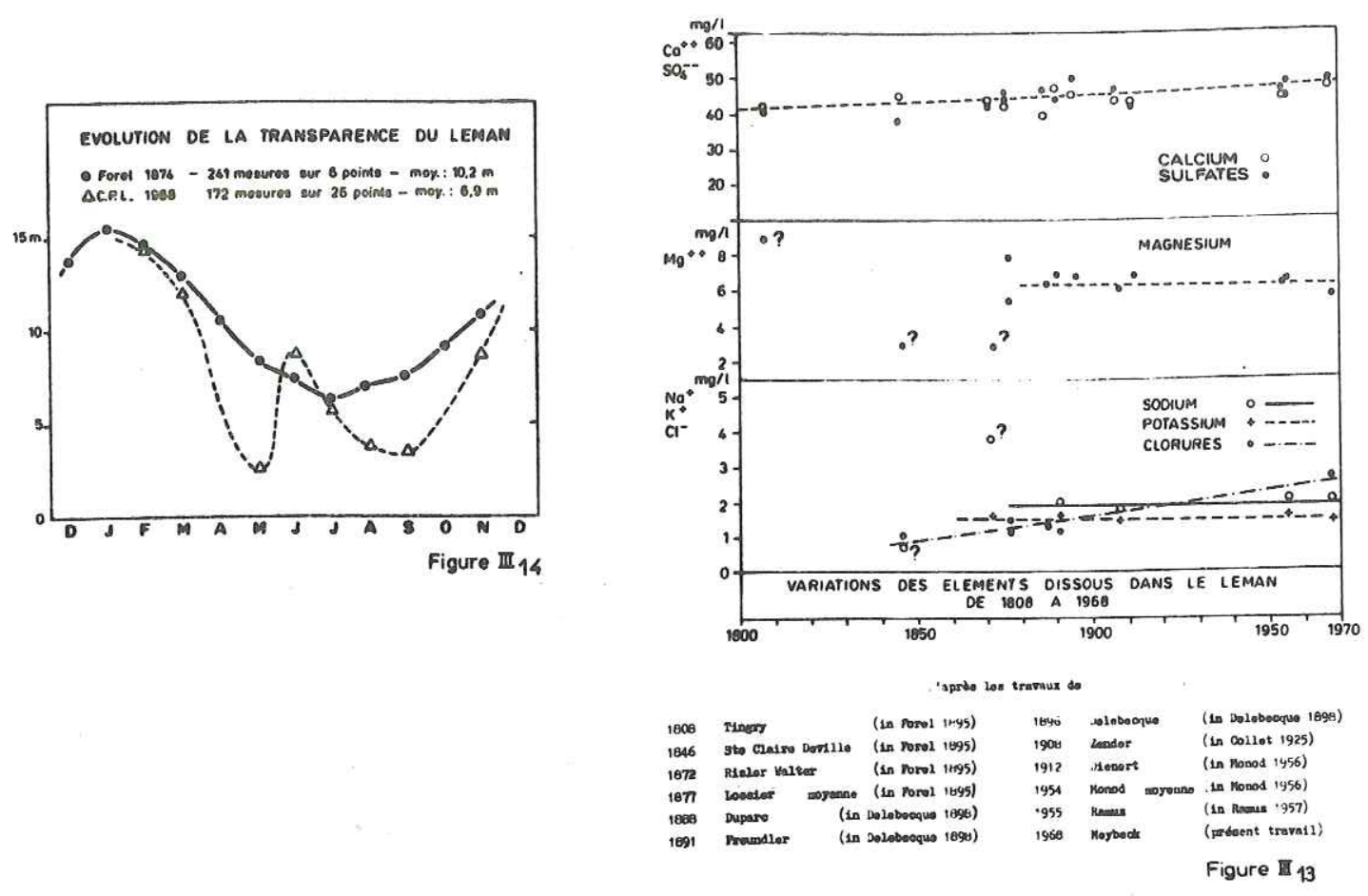

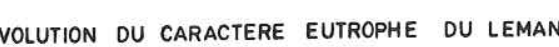

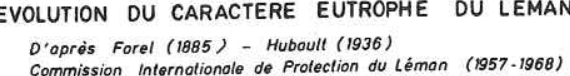

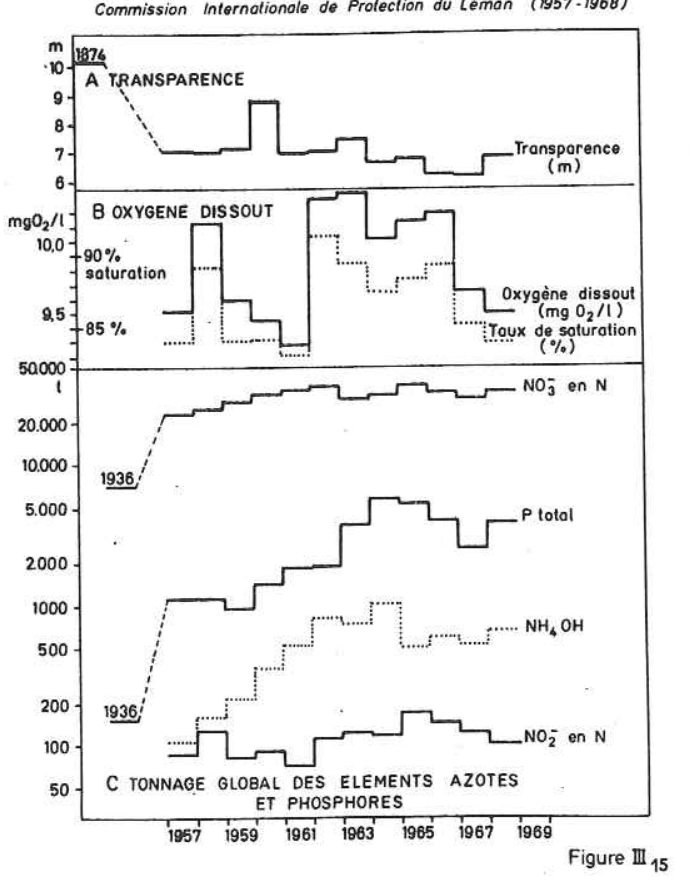

3.6.2.2. - oxygène.

Jusqu'à ces dernières années la répartition de l'oxygène dissout dans le Léman était orthograde, c'est-à-dire qu'il n'y avait pas de diminution importante de 1 'oxygène dans les couches profondes. Hubault (1947) donne des valeurs de près de $10 \mathrm{mg} / 1$ au fond du $1 \mathrm{ac}$ en 1936. Quand un lac s'eutrophise les couches supérieures (épilimnion) s'enrichìssent de plus en plus en oxygène jusqu'à dépasser le taux de saturation définì par la température et la pression, parallèlement les eaux profondes s'appauvrissent. Ce déséquilibre est de plus en plus net dans le Léman : la distribution d'oxygène a tendance à devenir clinograde, les eaux de fond ne sont plus saturées en oxygène qu'à $50 \%$, mais cette valeur peut être encore plus faible $(10 \%)$.

Nous avons porté sur la figure $\operatorname{III}_{15}$ l'évolution de la teneur moyenne globale en oxygène du Léman d'après la Commission de Protection du Léman. La concentration tend constamment à baisser et c'est seulement grâce à des hivers longs et rigoureux que les eaux du lac peuvent se réoxygéner. C'est le cas pour 1'hiver 1962-1963 (Monod 1965).

\subsubsection{3. - Eléments nutritifs.}

La cause princípale du développement très important du plancton est l'augmentation des teneurs dans le lac d'éléments nutritifs azotés et phosphatés indispensables à son développement. La provenance de ces éléments est multiple : naturelle (sels minéraux), agricole (engrais azotés et phosphatés), mênagère (détergents phosphatés) enfin industrielle et urbaine. Depuis 1957 1a Commission de Protection du Léman a pour tâche principale dans ses études chimiques d'effectuer le bilan de ces éléments (apports des rivières, valeurs moyennes du $1 \mathrm{ac}$, sorties à Genève). Nous avons reporté sur la fígure $\operatorname{III}_{15}$ l'évolution des tonnages globaux de tout le lac en nitrates, 
nitrites, ammoniaque et phosphates : en comparant avec les valeurs en 1936 estimées d'après les données d'Hubault (1947) on constate les faits suivants :

- En 30 ans, de 1936 à 1957, la masse de nitrates dans le Léman est passée de $7000 \mathrm{t}$ à $23000 \mathrm{t}$ d'azote équivalent, les phosphates sont passés de 150 t à 1100 t (phosphore total).

- De 1957 à 1963 il y a eu une augmentation importante des nitrates, phosphates et ammoniaque.

- Depuis 1963 la masse totale des éléments azotés est plus stable et oscille autour de $35000 \mathrm{t}$, de même pour les phosphates, il semble que 1 'augmentation se soit stabilisée depuis 6 ans.

I1 est donc certain que la très forte augmentation des êlêments nutritifs est à 1'origine d'un déséquilibre profond de la biologie du Léman. La masse totale de plancton s'est trouvée fortement augmentée et on note les changements qualitatifs de l'état biologique du Léman (Commission de Protection du Léman).

- Développement massif de macrophytes sur tous les bords du Léman.

- Changements qualitatifs dans la répartition des espèces planctoniques : nombre d'espèces recensées multiplié par 10 de 1957 à 1967, baisse nette de la proportion de diatomées, apparition d'osci1latoria rubescens en 1967 etc..

- Changements nets dans la faune du Léman : apparition et développement intense de dreissena polymorpha Pallas depuis 1962, prolifération de 1 'épinoche (Gasterosteus aculeatus L.) (d'après P. Laurent 1966).

Il faut remarquer que cette évolution du Léman s'est déjà remarquée dans bien d'autres lacs périalpins. Ainsi la teneur en phosphates du Bodensee a brusquement augmenté : de 0,003 mg/1 vers 1950 elle est passée à $0,025 \mathrm{mg} / 1$ (Mü11er, 1967). Dans 1 lac des quatre Cantons la concentration moyenne est passée de $0,012 \mathrm{mg} / 1$ en 1962 à $0,020 \mathrm{mg} / 1$ en 1968 (Ambühl, 1969). Le phosphore est considéré pour ces lacs comme le facteur essentiel du développement du plancton.

Depuis 1965 il semble que la quantitế globale d'éléments nutritifs a cessé d'augmenter dans le Léman, mais nous verrons la dífficulté de l'établissement des billans, de plus, comme dans le même temps la quantité totale de matière vivante dans le lac s'accroît énormément, il semble donc que le lac continue son êvolution vers 1'eutrophisation. Compte tenu du temps de renouvellement du Léman, la réponse du lac à une quelconque impulsion sera très lente, inversement la réaction du $1 \mathrm{ac}$ à des mesures de protection et d'asainissement sera également très longue.

\subsection{3. - Comparaison du Léman et des Grands Lacs du St-Laurent.}

Les Grands Lacs américains sont sans doute parmi les mieux étudiés depuis des dizaines d'années et leur évolution est bien connue. Dans son excellente étude Beeton (1965) arrive aux conclusions suivantes : les lacs accusent à la fois une pollution chimique très forte et des changements biologiques importants. Sauf pour le Lac Supérieur, tous les grands lacs ont accusé depuis 1900 une minéralisation nette des eaux pour les éléments majeurs $\left(\mathrm{Ca}^{++}, \mathrm{Mg}^{++}, \mathrm{Na}^{+}, \mathrm{K}^{+}\right.$, $\left.\mathrm{SO}_{4}{ }^{-}, \mathrm{Cl}^{-}\right)$. Les lacs Erie et Ontario ont vu leurs concentrations globales passer de $140 \mathrm{mg} / 1$ à $190 \mathrm{mg} / 1$ en 60 ans. Citons encore :

- lac Michigan : $\mathrm{SO}_{4}{ }^{-}$passe de $7 \mathrm{mg} / 1$ à $18 \mathrm{mg} / 1$.

- $1 \mathrm{ac}$ Huron : $\mathrm{SO}_{4}{ }^{-}$passe de $7 \mathrm{mg} / 1$ à $15 \mathrm{mg} / 1$.

- 1ac Ontario : $\mathrm{Ca}^{++}$passe de $30 \mathrm{mg} / 1$ à $38 \mathrm{mg} / 1$. $\mathrm{Cl}^{-}$passe de $7 \mathrm{mg} / 1$ à $25 \mathrm{mg} / 1$. 
L'interprétation de ces données est compliquée du fait que les lacs se dêversent les uns dans les autres mais Beeton attribue ces énormes changements au développement et à l'industrialisation de toute cette région. Il y a là un exemple frappant de pollution chimique effectuée par le peuplement humain. De plus les teneurs en oxygène dissout sont maintenant très faibles dans certains lacs -moins de $3 \mathrm{mg} / 1$ au fond du lac Erie-. Les changements biologiques quantitatifs et qualitatifs sont très visibles également : Si 6,8 millions de $\mathrm{kg}$ de brochets bleusse prenaient chaque année dans le lac Erie avant 1950, seuls quelques spécimens très âgés étaient encore pris en 1963 !

Il y a donc une différence radicale entre le Léman et les Grands Lacs : ces derniers ont été essentiellement pollués par des déversements d'eaux industrielles usées qui ont entre autres complètement changé leur équilibre chimique. Pour le Léman par contre aucun changement important dans la composition des éléments majeurs ne s'est produit depuis 100 ans; mais 1 'augmentation catastrophique des substances nutritives azotées et phosphatées est à l'origine d'un développement intense du plancton et de changements biologiques, signes de 1'évolution du Léman vers l'eutrophisation.
B - BILAN DES ELEMENTS SOLUBLES DU BASSIN DU LEMAN

\section{7. - Problèmes posés par l'établissement du bilan.}

\subsection{1. - Les termes du bilan.}

Plusieurs éléments entrent en ligne de compte pour effectuer le bilan des éléments dissous d'un lac à son émissaire. Les apports sont les suivants :

- rivières arrivant au $1 \mathrm{ac}$

- sources sous-lacustres

- ruissellement direct

- égouts, rejets de stations d'épuration, etc...

- précipitations atmosphériques directes sur le lac et retombées sèches

- dissolution d'éléments en suspension dans le lac

- diffusion à partir des eaux intersticielles des vases

les retraits sont les suivants :

- émissaire

- pertes sous-1acustres

- pompages de 1'eau du lac avec rejet en dehors du bassin

- évaporation et formation d'aérosols

- précipitation chimique

- précipitation biologique (plancton, animaux morts, etc...) 
On voit tout de suite la complexitê d'une telle étude, néanmoins on peut éliminer les sources et les pertes sous-lacustres qui sont très faibles sinon nulles dans le Lẻman (Blavoux, Dussart, Maneglier, Salvetti, 1962). Le déficit du bilan hydrique d'une année moyenne est de 1 'ordre de $10 \mathrm{~m}^{3} / \mathrm{s}$, il correspond au ruisse1lement direct et sans doute aux nappes alluviales du Rhône, de la Dranse et des autres affluents. Les prêcipitations directes sur le Léman sont de $1^{\prime}$ ordre de $1000 \mathrm{~mm} / \mathrm{an}$, ce qui équivaut à un apport moyen annuel d'environ $20 \mathrm{~m}^{3} / \mathrm{s}$. Les eaux de pluie sont de 1 'ordre de 10 à 50 foîs plus faîblement minéralisées que les rivières du Léman. Henson et al (1969) ont estimé que, dans le bilan du lac Champlain (U.S.A.), les proportions des apports d'origine atmosphérique vont de $0,26 \%$ pour le magnésium à $1,21 \%$ pour les chlorures. Cet ordre de grandeur est bien en dessous des incertitudes que nous avons ici sur les apports des rivières. Il en est de même pour l'évaporation et la formation d'aérosols chargés en ions dissous : leur influence sur le bilan doit être encore plus minime. Si les pompages d'eau du lac avec rejet en dehors du bassin versant (cas du Canton de Genève) ne sont pas très importants par rapport au bilan, il n'en est malheureusement pas de même du ruissellement direct et des rejets d'eaux usées : ces deux facteurs sont très difficiles à mesure et bien que les masses d'eau usées soient faibles les concentrations peuvent être très élevées, surtout pour les éléments azotés et phosphatés. Quant à la diffusion à partír des vases, les expériences en cours ( $\mathrm{P}$. Blanc, M. Meybeck) montrent que les eaux intersticielles sont beaucoup plus minéralisées que les eaux de fond. Nous avons vu que seule la silice présente un taux d'augmentation accru dans les couches profondes (voir sur la figure III $_{11}$, CRG 10 en Novembre 1967) pouvant être expliqué par une diffusion à partir du fond. Les études en cours permettront de préciser l'ampleur du phénomène pour chaque élément. Dans l'état actuel de nos connaissances nous ne savons rien sur la dissolution possible des matières en suspensions.

En effectuant donc la différence des apports des rivières et des sorties à Genève nous obtiendrons par défaut la quantitê de matières dissoutes consommées par les organismes vivants et précipitée chimiquement. La précision du bilan permettra de dire si ces différences sont significatives ou non.

\subsection{2. - Période de calcul du bilan}

Dans tout bilan il faut choisir comme limites des périodes de faibles variations des paramètres, nous avons choisi ici Janvier et Décembre. De plus il importe de tenir compte de la variation de tonnage global dans le lac entre ces deux limites, en effet le Léman ayant un temps de renouvellement moyen de 11 ans, les eaux sorties en Décembre n'ont rien à voir avec les eaux arrivées au mois de Janvier précédent. On peut donc faire : soit un bilan entrées-sorties sur une longue durée, sans faire de mesures sur le lac, soit un bilan sur une durée plus faible en tenant compte de la variation des teneurs du lac pendant cette période. Nous avons choisi cette deuxième méthode.

\section{8. - Bitan}

\subsection{1. - Bizan sans tenir compte de la variation du lac.}

Pour ce bilan de Janvier 1968 à Décembre 1968, les apports du Rhône et de la Dranse sont tels que nous les avons calculés au chapitre II. Pour les autres rivières affluentes qui représentent moins de $15 \%$ des apports directs nous avons utilisé les données de la Commission de Protection du Léman pour les êléments suivants : $\mathrm{Ca}^{++}, \mathrm{Mg}^{++}, \mathrm{Cl}^{-}, \mathrm{NO}_{3}{ }^{-}$. Les éléments restants $\left(\mathrm{SiO}_{2}, \mathrm{Na}^{+}, \mathrm{K}^{+}, \mathrm{SO}_{4}{ }^{-}-\right.$ $\mathrm{HCO}_{3}{ }^{-}$) ont été calculés par extrapolation en assimilant ces petites rivières à la Dranse et à la Venoge de compositions chimiques bien connues. Pour les sorties à Genève nous avons pris la moyenne arithmétique en 1968. Le tableau $\operatorname{III}_{13}$ représente le bilan. On note un déficit hydrique de $21 \mathrm{~m}^{3} / \mathrm{s}$ provenant du ruissellement direct, des égouts, et de la différence pluie-êvaporation. Ce bilan est donc largement 
donné par défaut. Il semble que les différences pour la silice et les nitrates sont significatives. Nous allons voir ce qu'il y a lieu d'en penser.

3.8.2. - Bilan en tenant compte de la variation de composition des eaux du lac.

Nous avons estimé la masse totale pour chaque élément contenu dans le Léman en Janvier 1968 et en Décembre 1968 en milliers de tonnes.

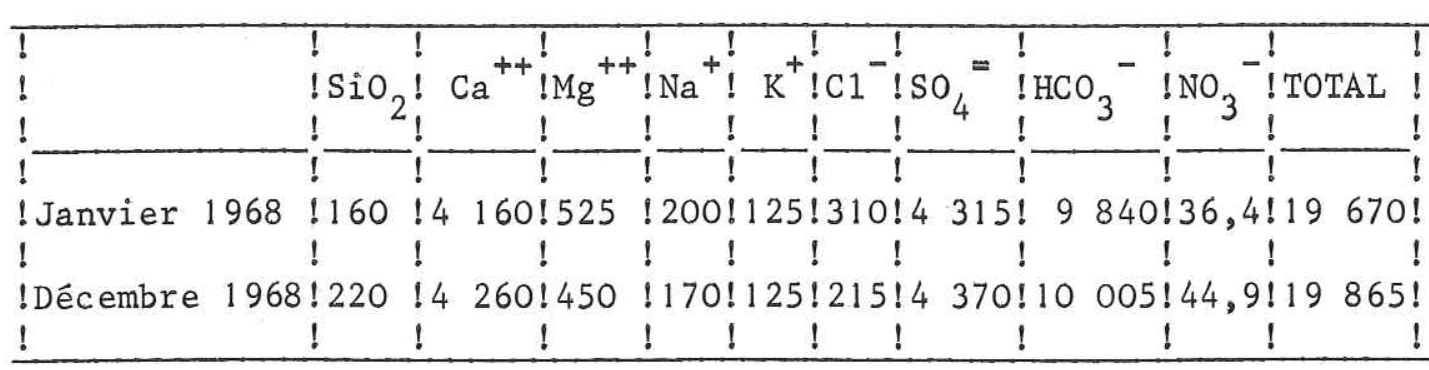

Les variations du magnésium, des chlorures et du sodium son suspectes, en effet, elles peuvent être dues aux erreurs d'analyses ou même aux changements de méthode. Par contre il apparaît nettement que les concentrations en silice ont augmentées de $30 \%$ et celles des nitrates de $20 \%$, c'est-à-dire 60000 t supplémentaires de silice et $8500 \mathrm{t}$ de nitrates en azote équivalent. Les autres éléments, $\mathrm{Ca}^{++}$, $\mathrm{SO}_{4}{ }^{-}$- et $\mathrm{HCO}_{3}{ }^{-}$n'ont varié que de moins de $2 \%$ maîs ces variations représentent quand même $100000 \mathrm{t}$ pour le calcium, $55000 \mathrm{t}$ pour les sulfates et 165000 t pour les bicarbonates.

Le bilan précédent que nous avons effectué n'est donc plus significatif : une petite variation de $0,1 \mathrm{mg} / \mathrm{l}$ pour l'ensemble du Léman suffit à équilibrer une augmentation de $1 \mathrm{mg} / \mathrm{l}$ des concentrations moyennes des apports.

\subsection{3. - Bilan sur plusieurs années.}

Nous pouvons essayer de faire un tel bilan avec les phosphates et les nitrates grâce aux données fournies par la Commission de Protection du Léman sur une durée de cinq ans, de 1964 à 1968 :

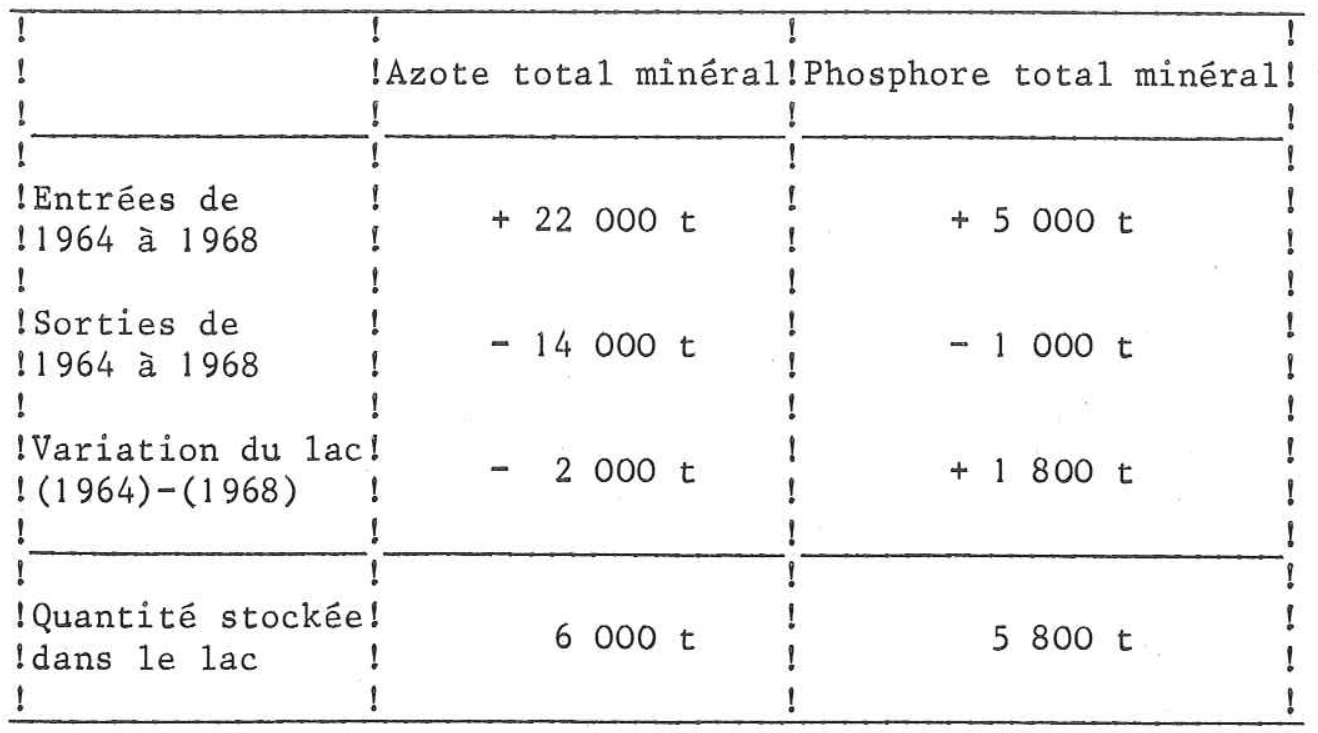

Ce bilan n'est bien sûr qu'indicatif puisqu'il devrait porter sur au moins 11 ans, mais on peut quand même avancer que le lac s'est enrichi (biomasse, sédiments) d'environ $6000 \mathrm{t}$ d'azote total minéral et 5800 t de phosphore total minéral en cinq ans.

\section{9. - Erosion chimique du bassin versant du Léman.}

Si on considère que la surface du bassin versant fermé au Pont du Mont-Blanc à Genève est de $7975 \mathrm{~km}^{2}$, et en prenant les concentrations moyennes à Genève sur deux ans on aura les valeurs suivantes comparées au Bodensee (tableau $\operatorname{III}_{14}$ ) 
TABLEAU III $14:$ EROSIONS SPECIFIQUES COMPAREES DES BASSINS VERSANTS DU LEMAN ET DU BODENSEE

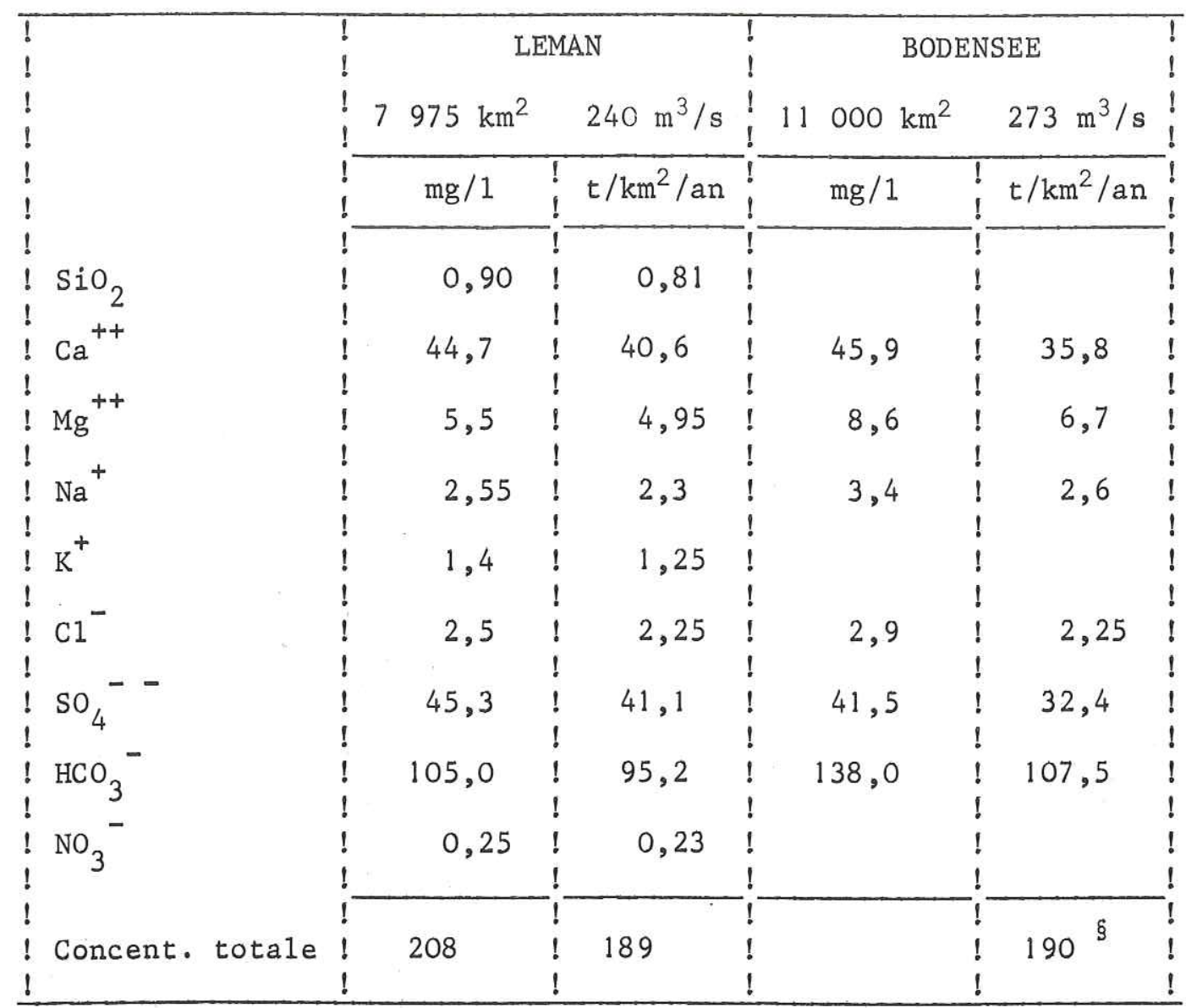

$\S$ - valeur extrapolée.

Les érosions spécifiques sont calculées d'après les données de Müller (1964) pour 1e Rhin lacustre ou Seerhein, émissaire $\mathrm{du}$ Bodensee (Obersee). Une fois encore la similitude géochimique des deux lacs est frappante.

On peut donc estimer que l'érosion chimique moyenne sur le versant nord des Alpes est de $190 \mathrm{t} / \mathrm{km}^{2} /$ an. Il est intéressant de comparer cette valeur avec celle du Pô. D'après L. Canalli (1964) le Pô amène chaque année à la mer 11,5 mil1ions de tonnes d'éléments dissous. Pour un bassin versant de $70000 \mathrm{~km}^{2}$ cela correspond à une érosion spécifique chimique de $16,5 \mathrm{t} / \mathrm{km}^{2} / \mathrm{an} c^{\prime}$ est-à-dire 10 fois moins que celle du versant nord des Alpes. Remarquons que l'érosion chimique pour 1a Bregenzer Ach, deuxième affluent du Bodensee et aussí semblable à la Dranse que le Rhin 1'est au Rhône, est de $535 \mathrm{t} / \mathrm{km}^{2} / \mathrm{an}$. Nous voyons au chapitre IV (tableau $\mathrm{IV}_{2}$ ) que ces valeurs sont très fortes.

\subsection{0. - Conclusion générale de l'étude et du bilan chimique des} eaux du Léman.

Nous nous étions posé beaucoup de questions méthodologiques au début de ce chapitre, nous pouvons maintenant tenter d'y répondre. Les variations principales des composants dissous du Léman sont fonction de la profondeur, seules les couches supérieures sont soumises aux importantes variations saisonnières. Dans 1 'hypolimnion les variations sont très atténuées, seuls quelques éléments y varient encore comme les bicarbonates, calcium et silice qui augmentent régulièrement vers $1 \mathrm{e}$ fond. Les variations géographiques sont secondaires dans le Léman.

Dans l'état actuel de nos connaissances il n'est pas possible d'atteindre dans l'établissement des bilans d'éléments dissous une grande précision. D'abord il est nécessaire de connaître avec précision les variations des teneurs dans le lac : en effet vu l'énorme volume du Léman une petite variation de la concentration générale peut rendre compte d'une grosse différence entre les apports des rivières et la sortie de 1'émissaire. Il suffira donc pour avoir une bonne idée de l'état du lac de choisir 4 ou 5 stations d'études, d'y effectuer des prélèvements mensuels -les variations se produisent en effet sur plusieurs mois en généra1-, les profondeurs des prélèvements devant être $0-10-20-50-100-150 \mathrm{~m}$ etc... pour bien tenir compte des variations de l'épilimnion. Il faudra au moins une station au-dessus de la plaine centrale à $310 \mathrm{~m}$ de profondeur. 


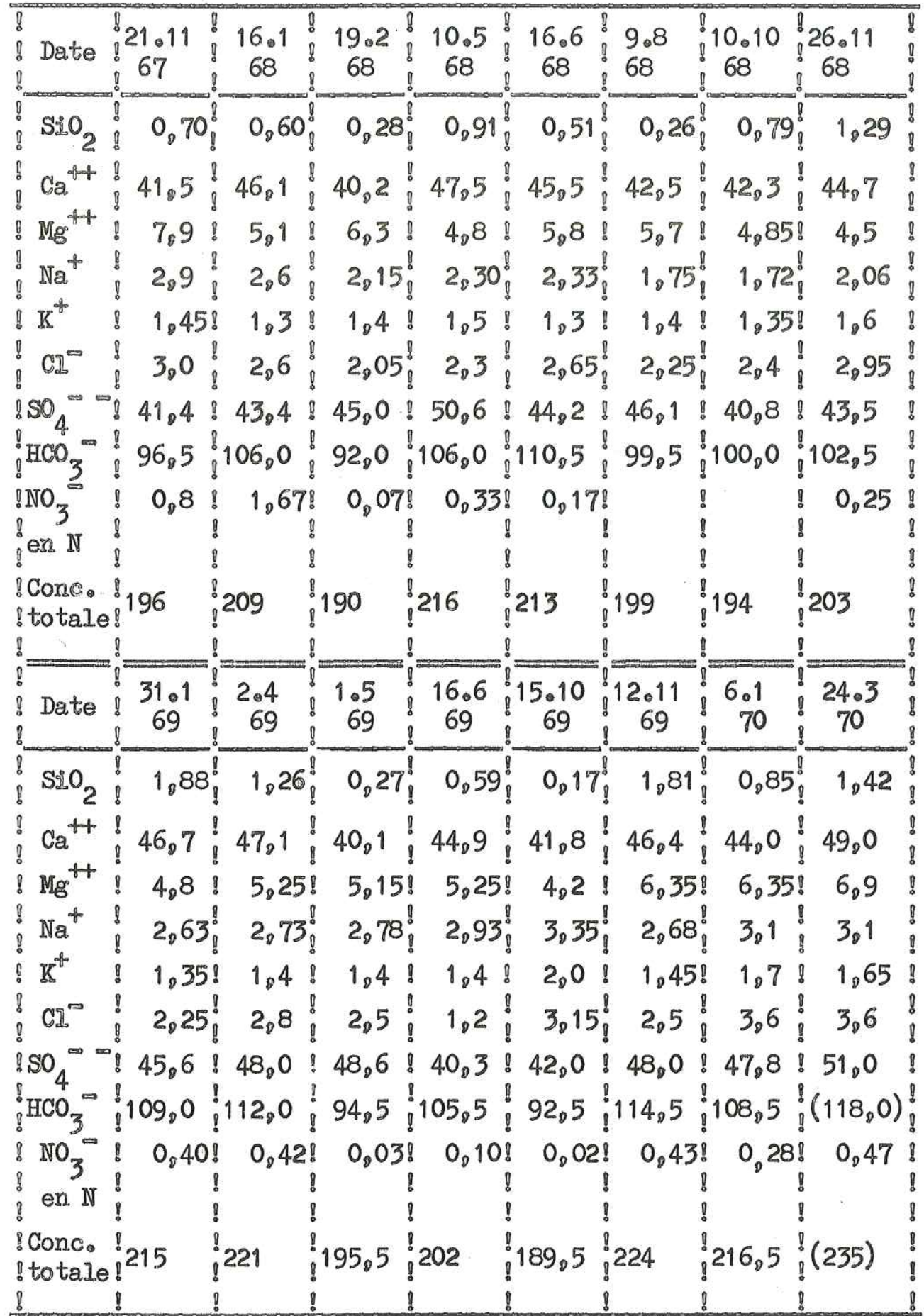

TABLEAU III

ANALYSES CHIMIQUES COMPLETES DU RHONE A LA SORTIT DU LAMAN (Genère) en $\mathrm{mg} / \mathrm{I}$

Remarques: - ( ) Valeurs extrapolées - Les nitrates sont donnés en mg N// précipitations du carbonate de calcium avant l'analyse. 


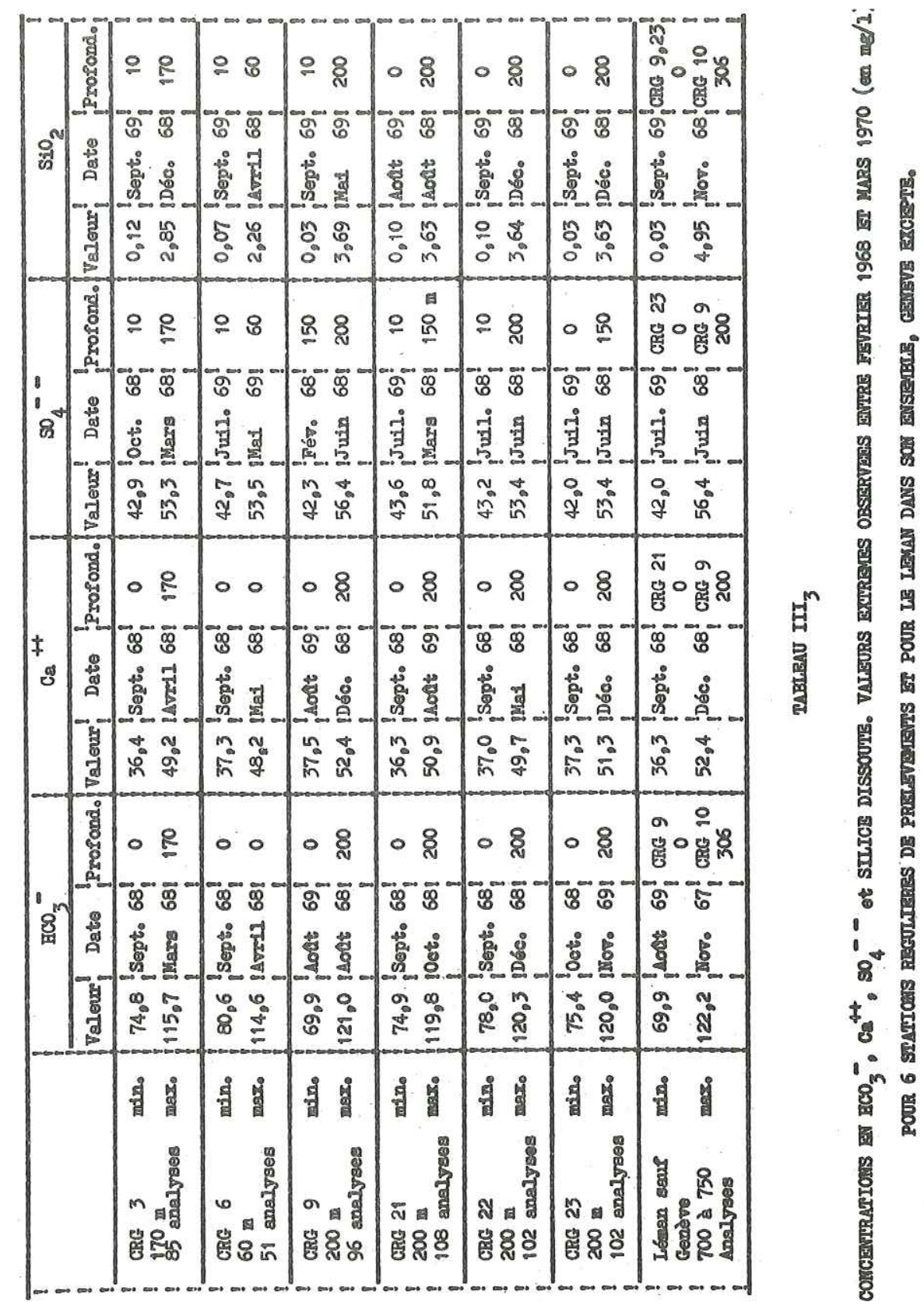

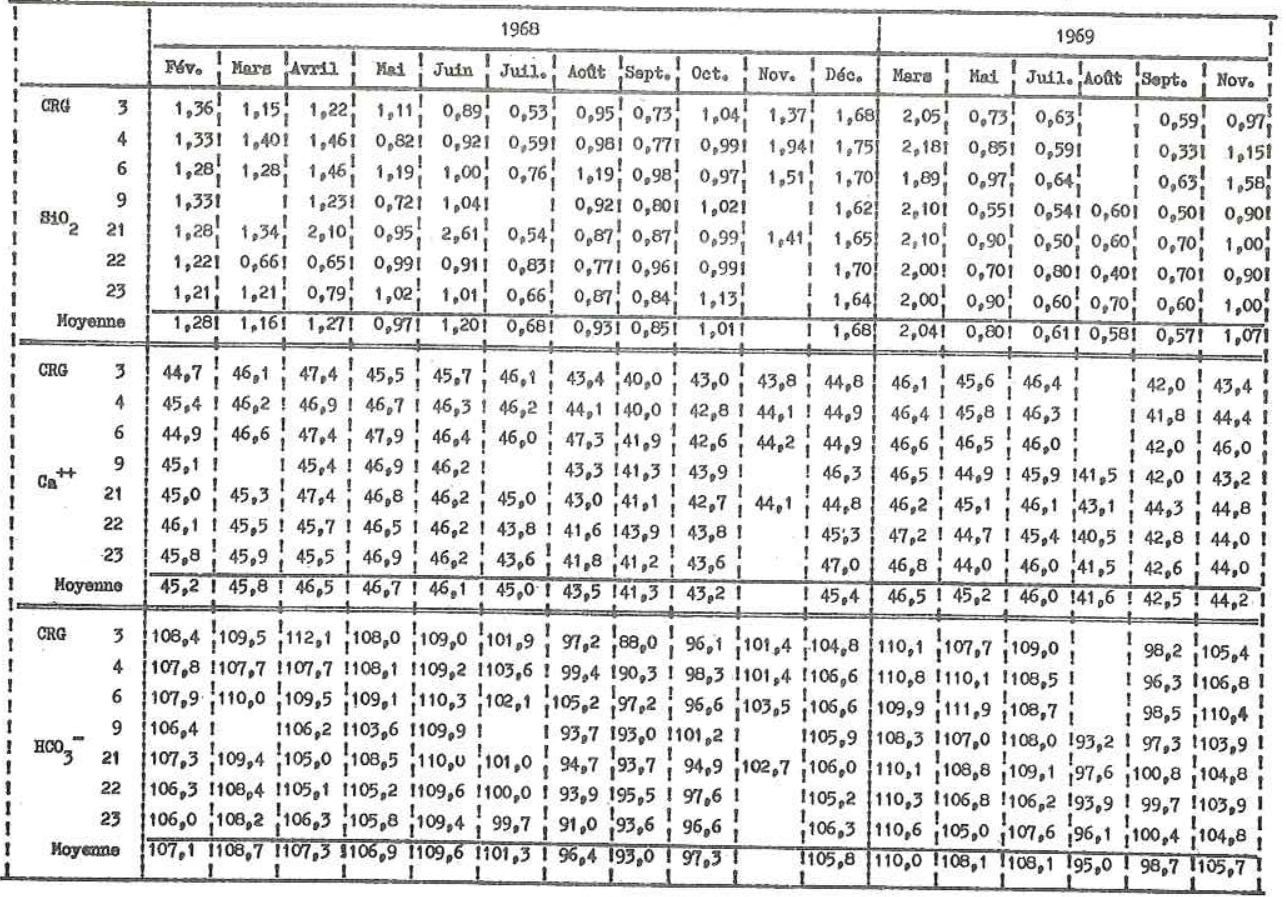

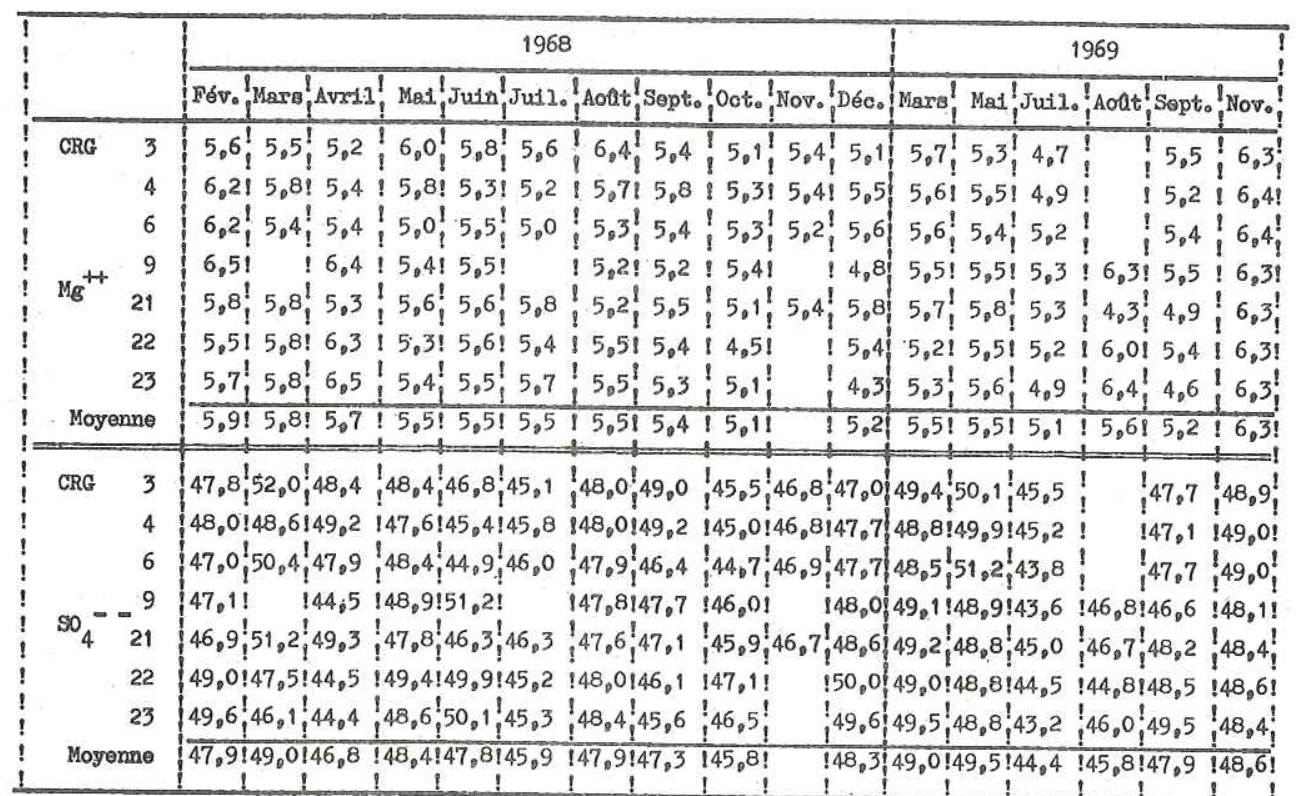

TABBLEN IIII

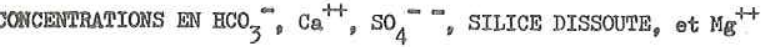

DANS IA COUCERE SUPRRPTCIELLE (MOYENNE DES VALEURS $0 \mathrm{~m}, 10 \mathrm{~m}$ et $50 \mathrm{~m}$ )

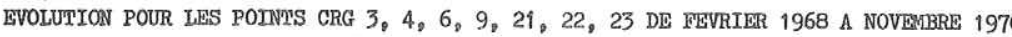




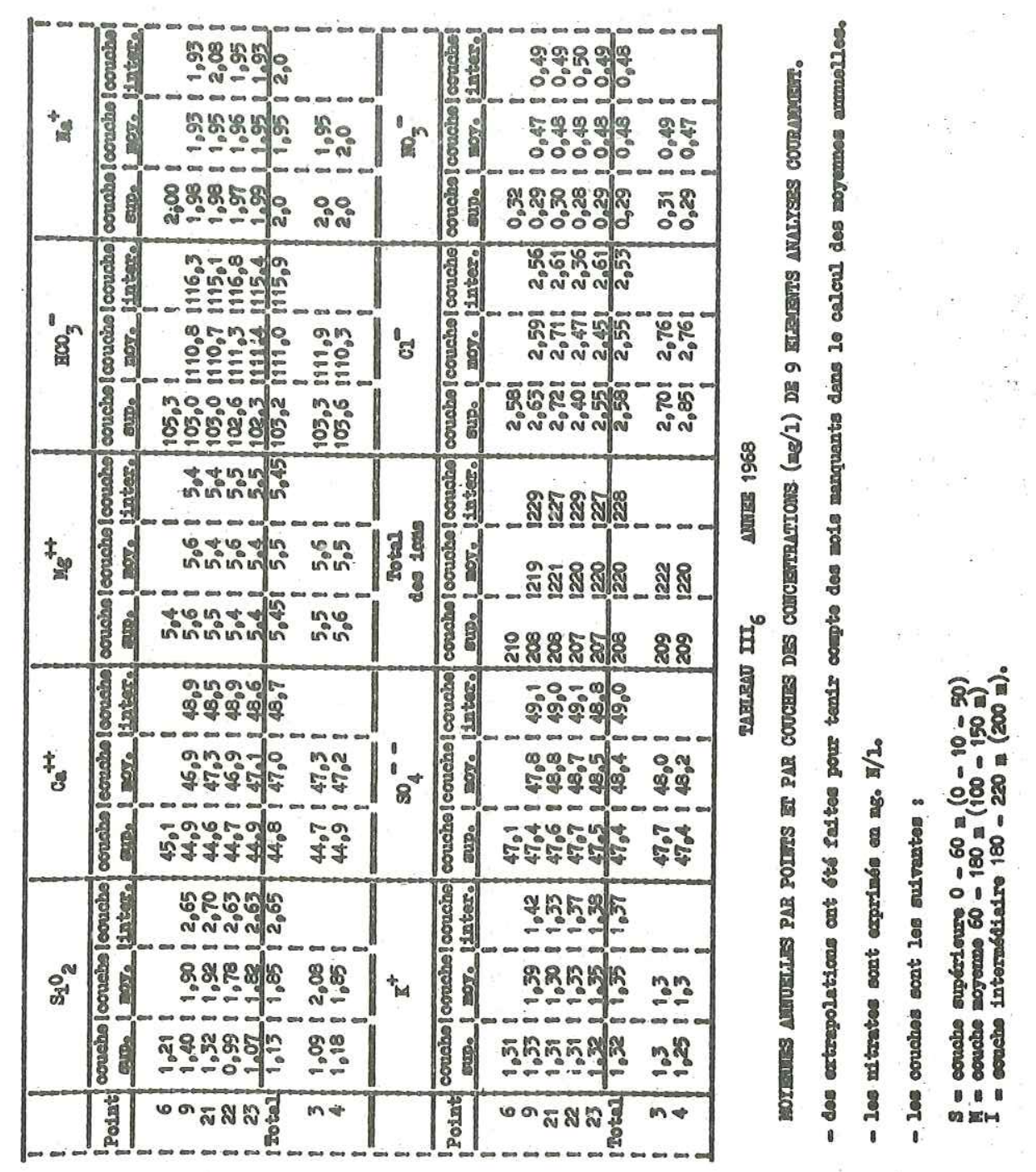

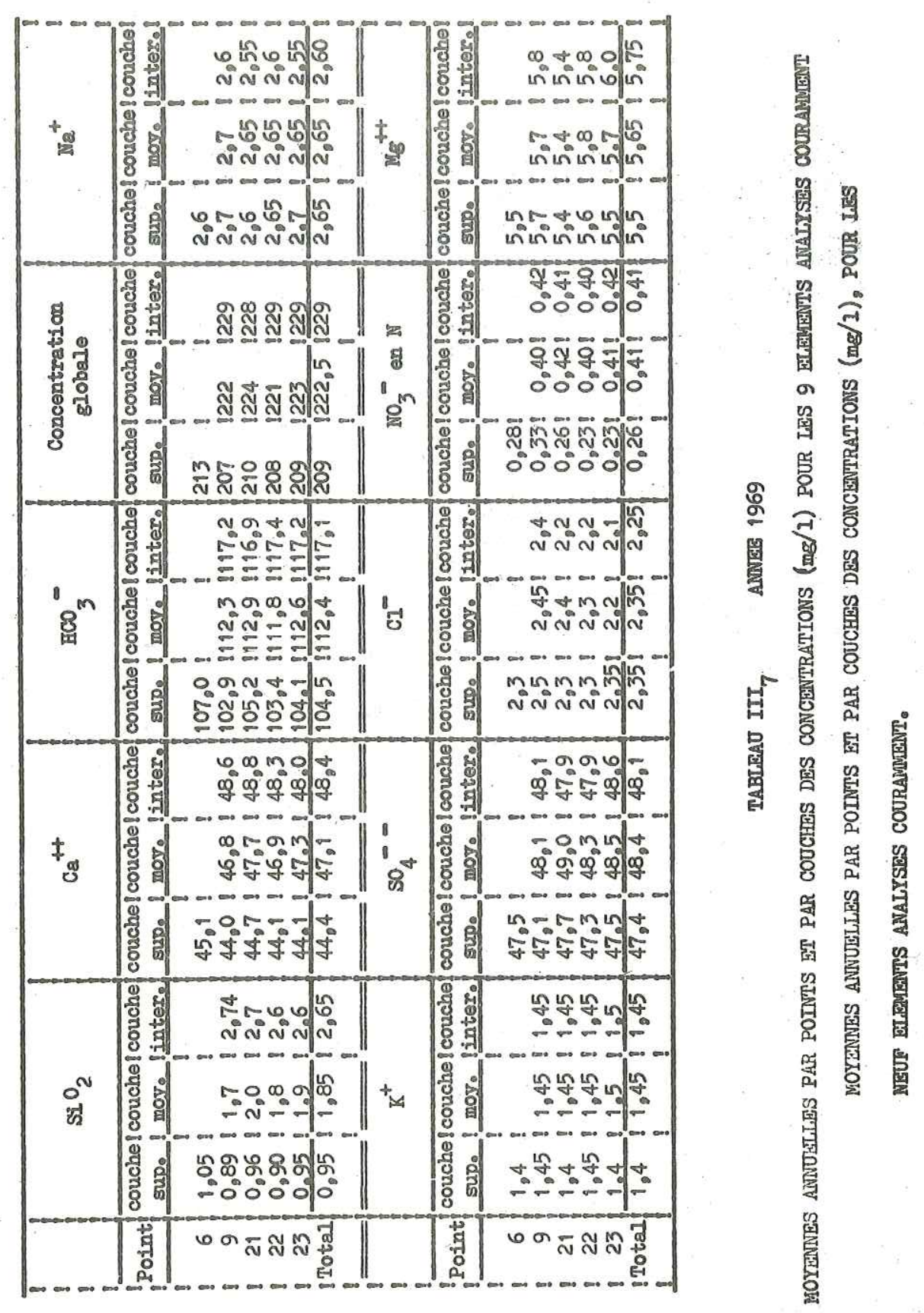




\begin{tabular}{|c|c|c|c|c|c|c|c|c|c|c|c|c|c|}
\hline \multirow{2}{*}{ Date } & 6 & \multicolumn{3}{|c|}{9} & \multicolumn{3}{|c|}{21} & \multicolumn{3}{|c|}{22} & \multicolumn{3}{|c|}{23} \\
\hline & gupe & & i & intinger & Esp. & $f$ mor & linter & вир, & 1 move & linter & supo & $1 \operatorname{mox}$ & ifintex \\
\hline Fovo & 215 & 214 & 215 & $\begin{array}{lll}7_{2}^{2} \\
\end{array}$ & 214 & 221 & \begin{tabular}{|l|l}
1229 \\
\end{tabular} & 215 & \begin{tabular}{|lll}
220 \\
\end{tabular} & $\frac{1}{3226}$ & 216 & 218 & \\
\hline Mars & | 220 & 1 & i / & 1 & 219 & i 220 & i 226 & 214 & i 216 & : 2225 & 214 & 216 & 220 \\
\hline Avri2 & 218 & 210 & 215 & 217 & 225 & 219 & : & | 208 & ?215 & $\{225$ & 200 & 214 & 218 \\
\hline Mas & 218 & 213 & 222 & 2228 & 217 & i 222 & 225 & 214 & 221 & I230 & 214 & 229 & 229 \\
\hline Juin & 215 & 2 & ; 222 & 240 & 218 & 218 & 225 & 219 & ? 223 & 234 & 219 & 225 & 236 \\
\hline & 206 & & 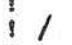 & & & 220 & 2200 & 201 & ? 221 & 226 & 200 & 220 & 226 \\
\hline Aoat & 210 & 197 & 220 & 224 & 198 & 220 & 231 & 196 & 2224 & 231 & 194 & 224 & \\
\hline Septo & 197 & 194 & 219 & 2229 & 194 & 221 & 226 & 197 & I 220 & 1 229 & 192 & 222 & 225 \\
\hline oct. & 195 & 203 & 223 & 228 & 195 & 221 & : 231 & 199 & \begin{tabular}{|l}
$\vdots 221$ \\
2
\end{tabular} & 230 & 199 & 221 & 231 \\
\hline & 207 & & i / & 1 & 206 & i 221 & i 227 & I & i / & $i /$ & 1 & / & \\
\hline Déo. & 209 & 212 & 224 & 233 & 213 & 223 & 231 & 214 & 225 & 235 & 215 & 223 & 234 \\
\hline $\begin{array}{c}\text { Mogonne } \\
\text { 19668 }\end{array}$ & 210 & 208. & 219 & 228 & 208 & | 221 & i 227 & 207 & i 220 & 229 & 207 & 220 & 227 \\
\hline Mara & 220 & 29 & 222 & 234 & 220 & 222 & 224 & 220 & \}$_{223}$ & 228 & 27 & 222 & 225 \\
\hline Mai & 223 & & \begin{tabular}{|l}
223 \\
223
\end{tabular} & 23 & & 223 & 228 & & 22 & 228 & & & \\
\hline 69 Juil. & 211 & 210 & 220 & 228 & 212 & 223 & 230 & 209 & 216 & \begin{tabular}{|l|l}
224 \\
\end{tabular} & 208 & 219 & 223 \\
\hline & 1 & 1 & 219 & 225 & & 223 & 231 & 15 & 219 & 225 & 197 & 226 & 232 \\
\hline & 201 & & : 226 & 232 & & $\{225$ & ? 226 & 204 & : 224 & 237 & 205 & 223 & 233 \\
\hline Novo & 220 & 209 & 223 & 228 & 211 & 229 & 232 & 219 & 225 & 233 & 211 & 227 & 233 \\
\hline Mogenne & 213 & 207 & 222 & 229 & 210 & 224 & 228 & 208 & 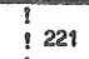 & 229 & 209 & 223 & 229 \\
\hline & & & & & & & 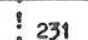 & 202 & & & & & \\
\hline
\end{tabular}

TABURAD IIIB

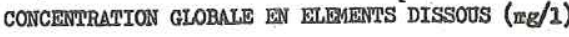

$\left(\mathrm{SsO}_{2}, \mathrm{Ca}^{++}, \mathrm{Mg}^{+{ }^{+4}}, \mathrm{Na}^{+}, \mathrm{K}^{+}, \mathrm{Cl}^{-}, \mathrm{SO}_{4}^{-}{ }^{-}, \mathrm{HCO}_{3}^{-}, \mathrm{CO}_{3}^{-{ }^{-}}, \mathrm{NO}_{3}^{-}\right)$

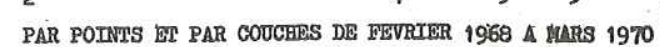

R⿴囗十 -11 n'a pas dote temu compte des gas diseouso

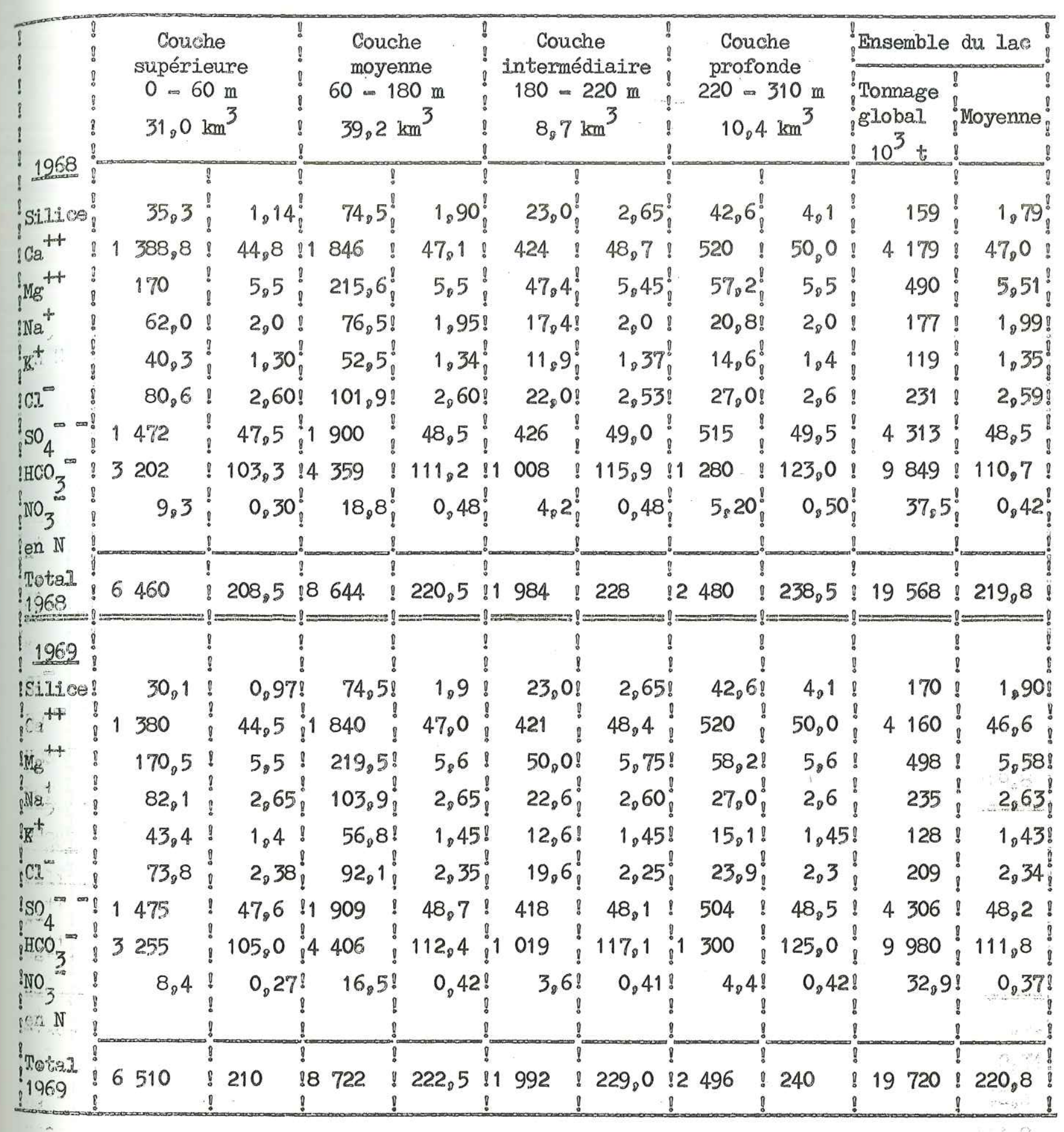

TABLEAUX III 9 et $\operatorname{III}_{10}$

LEMMAN : TENEUR MOYENNE EN ELENENTS DISSOUS EN 1968 ET 1969

1 ère colonne : tonnages partiels et gilobauss en milliders de tonnes 2àme colonne : concentrations moyennes en $\mathrm{mg} / \mathrm{I}$ ( $\mathrm{NO}_{3}{ }^{-}$exprimés en azote) 
TABLEAU III 11 : ANALYSE CHIMIQUE COMPLETE DU LAC LEMAN

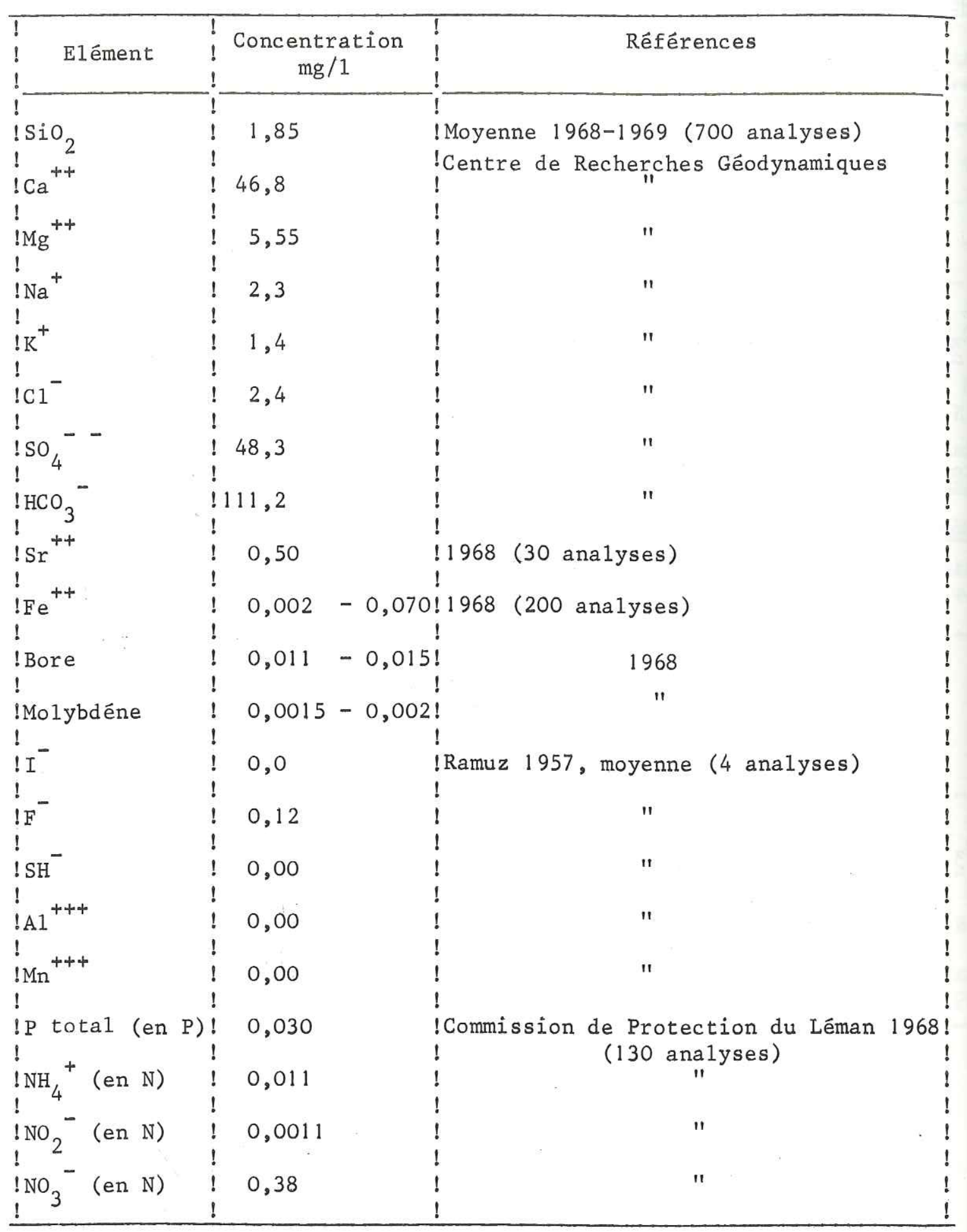

- 117 -

\begin{tabular}{|c|c|c|c|c|c|c|c|}
\hline ! & $\begin{array}{l}8 \\
0 \\
0 \\
8 \\
1 \\
8 \\
8 \\
0\end{array}$ & Rhône & $\begin{array}{ll}8 & \\
8 & \\
8 & \text { Dranse } \\
8 & \\
8 & \\
8 & \\
\end{array}$ & 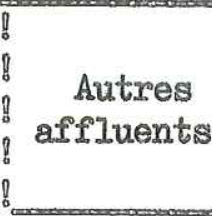 & $\begin{array}{l}\text { : } \\
\vdots \\
\vdots \\
\vdots \\
\vdots\end{array}$ & Genève & $\begin{array}{l}\text { I } \\
\text { 1. Différence } \\
\text { o (apports) } \\
\text { o- (sorties) } \\
\text { b }\end{array}$ \\
\hline 列 & $!$ & & $:$ & ! & $!$ & 8 & 8 \\
\hline $\mathrm{S}_{2} \mathrm{O}_{2}$ & $!$ & 16700 & 2600 & 3500 & 22800 & 4. 900 & $8+18000$ \\
\hline : $\mathrm{C}_{3}^{+2+t}$ & $i^{\circ}$ & 232000 & 53300 & 1170000 & 402000 & 330000 & 80000 \\
\hline : & 8 & & $! \quad c=0$ & 8 & 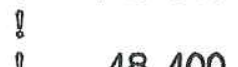 & $\frac{1}{8}$ & 879500 \\
\hline $\begin{array}{l}8 \\
8 \\
8\end{array}$ & $\begin{array}{l}0 \\
0 \\
1\end{array}$ & 28000 & 6300 & 14100 & 48400 & 48000 & $!+7500$ \\
\hline $\mathrm{Na}^{\mathrm{t}}$ & ! & 17000 & 1200 & 4400 & 22600 & 16500 & $i+6000$ \\
\hline$\pi^{+t}$ & $\frac{8}{8}$ & & 8 & 8 & $!$ & : & ! \\
\hline$K$ & $a^{0}$ & 7000 & 900 & 2600 & 10500 & 10500 & $\frac{1}{8}$ \\
\hline CI & $a^{2}$ & 17500 & 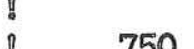 & 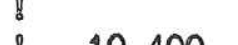 & ! & ! & $\frac{0}{10}$ \\
\hline$\pi=0$ & 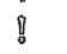 & ( & ! & $i$ & 00 & 10000 & $\begin{array}{lll}8 & 4 \\
\vdots\end{array}$ \\
\hline $\mathrm{SO}_{4}$ & $\frac{1}{8}$ & 296000 & 43500 & 44000 & 383500 & 335000 & $8+50000$ \\
\hline $\mathrm{HCO}_{3}=$ & 8 & 475000 & 144000 & 233000 & 852000 & 770000 & +70000 \\
\hline mo & ! & & ! & ! & $!$ & $!$ & 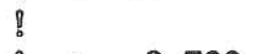 \\
\hline$e^{3} \pi$ & 8 & $(2600)$ & 380 & 1550 & 4500 & 1880 & $8+2500$ \\
\hline & ! & & 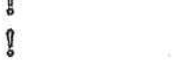 & 8 & $\frac{1}{8}$ & b & : \\
\hline Total & 81 & 090000 & \& 253000 & 430000 & $\begin{array}{l}0 \\
0\end{array}$ & \& 1530000 & $8+240000$ \\
\hline & $\frac{8}{8}$ & & $g^{8}$ & & & 8 & 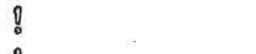 \\
\hline & ! & & 8 & 8 & $!$ & 8 & ! \\
\hline $\begin{array}{c}\text { \& Q annuel } \\
\text { : } 3 / \mathrm{s}\end{array}$ & $\begin{array}{l}8 \\
8\end{array}$ & 166 & $\frac{1}{8} \quad 23,6$ & 28 & 248 & 239 & $=21$ \\
\hline
\end{tabular}

BILAN GLOBAL (EN TONNES) DU LENAN POUR 1968 PAR ELEMVENTS, EFTECTUE SANS TENIR COMPTE DE LAA VARTATION DE TENEUR DU LAC. 


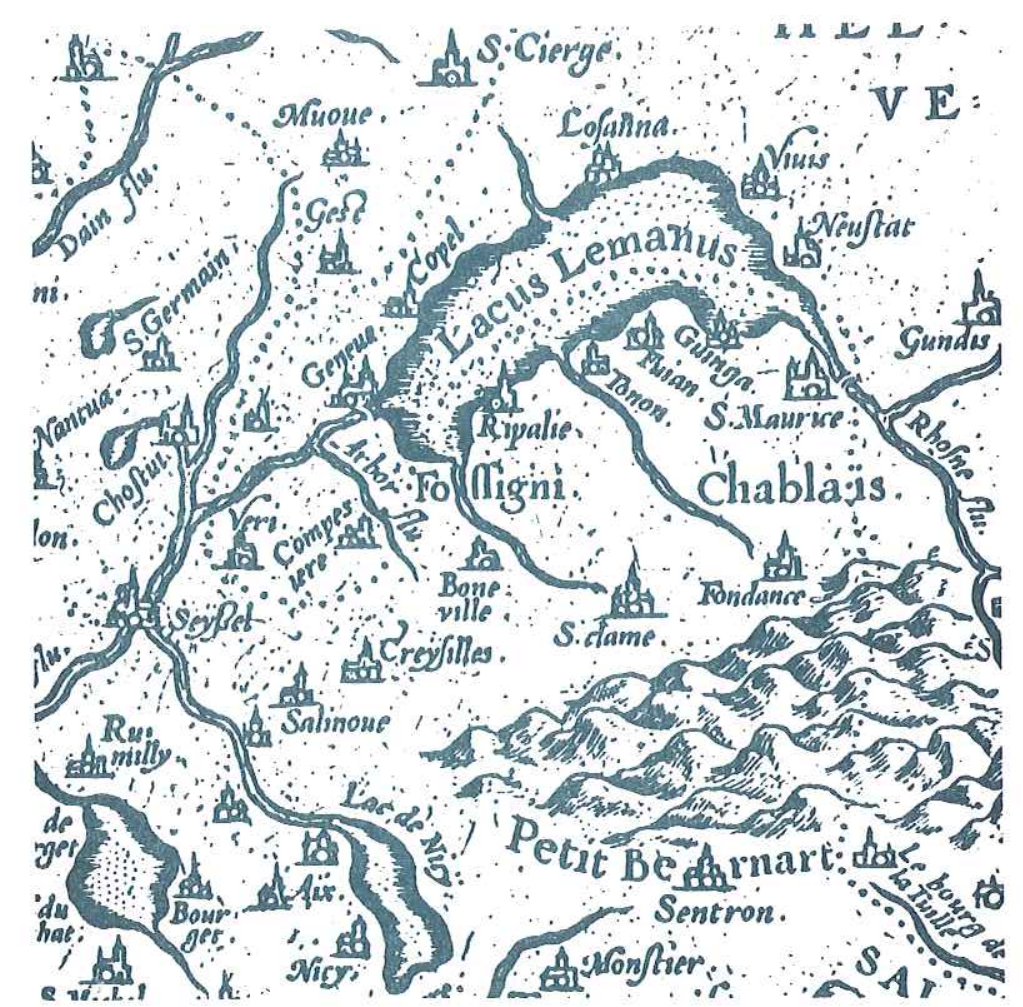

BILAN SEDIMENTAIRE ET

Univ. J. Fourier" "O.NCIENCE MAISON DES GEOSTION 
A - LES APPORTS TERRIGENES.

\section{1. - Erosion du bassin du Léman.}

\subsection{1. - Méthodologie.}

L'évaluation de la masse de matériaux détritiques apportés au Léman est fondamentale pour l'étude de la sédimentation actuelle du Léman, de plus elle nous donne des indications très utiles sur les taux d'érosions dans cette partie des Alpes. Néanmoins la mesure et le calcul de ces données sont très délicats et appellent les remarques suivantes.

4.1.1.1. - Mesure de turbidité.

Deux types différents de mesures existent :

1) Mesures discontinues par pesées.

Ces méthodes consistent à prendre un échantillon représentatif de la section, soit par prêlèvements multiples, soit par prélèvement intégré. L'échantillon est ensuite filtré, sèché et pesé.

2) Mesures en continu et in situ.

I1 y a actuellement trois possibilités :

- turbidimètre optique basé sur 1'absorption de la lumière 
émise par une source d'intensité connue. Cette méthode est surtout appliquée pour les faibles turbidités.

- sonde à transmission nucléaire, absorption ou diffusion du rayonnement gamma émis par une source connue (méthode ponctuelle).

- mesure de la radioactivité naturelle des suspensions par un détecteur placé in situ (méthode d'intégration). La radioactivité mesurée est proportionnelle à la turbidité. Cette méthode, qui nécessite un étalonnage précis, a été particulièrement développée ces dernières années par Martin (Martin, Meybeck, Heuze1, 1970), elle est surtout valable pour les très fortes turbidités allant jusqu'à que1ques centaines de $\mathrm{g} / \mathrm{l}$.

Dans le cas du Rhône seules des mesures par pesée ont été effectuées à notre connaissance à la station de la Porte de Scex. L'Office Fédéral de 1'Economie Hydraulique Suisse de Berne ${ }^{\S}$ effectue des prélèvements deux ou trois fois par semaine depuis 1964 sur plusieurs verticales à l'aide d'une bouteille spécialement mise au point par 1'Office Fédéral. Les eaux sont filtrées, puis le filtrat est séché à plus de $100^{\circ}$. Cet échantillonnage nous a été très utile pour étudier les lois des transports solides du Rhône.

Actuellement le débit solide sur le fond (traction load) est difficilement mesurable, depuis quelques années les méthodes de traceurs radioactifs ont fait beaucoup progresser cette mesure. Sur le Rhône nous ne disposons que des estimations fournies par le laboratoire d'Hydraulique de $1^{\prime} E P U L$ à Lausanne.

$\S$ Je tiens à remercier vivement Mrs Guenin et Kohler de 1'Office Fédéral de 1'Economie Hydraulique de Berne pour leur amabilité et les documents qu'ils ont mis à ma disposition.

\subsubsection{2. - Méthodes de calcul.}

Encore plus que pour le bilan des éléments dissous, les méthodes de calcul sont très importantes pour dêterminer le débit solide : 1'expérience montre que sur certaines rivières le débit solide peut atteindre en un ou deux jours de crue, $90 \%$ du débit annuel. La charge en suspension $(g / 1)$ est en effet extrêmement variable pour un même bassin versant. Si la variation principale est due au débit, comme pour la charge en solution, on note en plus des variations extrêmement fortes avec 1a rapidité de montée des eaux, la saison, la place de la crue dans la saison (les premières crues étant les plus turbides). Au sein d'une même crue, il y a en plus un décalage entre la montée et la descente des eaux : le maximum de charge en suspension se situe généralement avant le maximum du débit, vers le deuxième tiers de la montée des eaux ; les eaux de crue seront donc toujours plus chargées que les eaux de décrue. Enfin plus encore que pour les charges en solution, des évènements localisés dans un des sous-bassins comme les glissements de terrains, avalanches, rupture de poches glaciaires, auront des effets à l'exutoire très importants sans qu'on puisse toujours analyser ces augmentations de turbidité. La plupart du temps les auteurs ne communiquent pas leurs méthodes de calcul, pour le Rhône les méthodes suivantes ont été employées :

- Forel (1895). Cet auteur avait déjà remarqué la disparité entre les charges en suspension des crues et des décrues. Il dresse donc deux correspondances (Le Léman, tome I, p. 375) entre les débits liquides et la charge en suspensions, en crue et en décrue. Cette distinction est déjà excellente mais malheureusement dans son calcul du bilan pour 1886 Forel prend les débits moyens mensuels, les effets des crues sont donc complètement effacés.

- Serruya (1969). A partir des données de 1'Office Fédéral de 1'Economie Hydraulique, Serruya calcule la moyenne mensuelle des 
charges solides puis multiplie ces moyennes par les débits moyens mensuels. Les crues étant pour le Rhône étalées sur quelques jours (voir figure $\mathrm{II}_{6}$ ), cette méthode appelle les mêmes remarques que celle de Forel. Mais contrairement au savant vaudois Serruya "n'a jamais constaté aucune relation valable entre la charge solide et le débit instantané" et il lui semble "hazardeux de déterminer la charge solide d'après le débit du fleuve pour des périodes inférieures au mois". Nous verrons plus loin ce qu'il y a lieu de penser d'une telle affirmation.

Nous sommes amenés à faire les remarques suivantes sur les bilans d'éléments en suspension: et filtr

1) Méthodes discontinues de turbidimétrie (prélèvement

Comme les charges en suspensions Cs $\left(\mathrm{kg} / \mathrm{m}^{3}\right)$ varient en fonction des débits liquides $Q_{1}\left(\mathrm{~m}^{3} / \mathrm{s}\right)$, il ne faut $j$ amais effectuer des moyennes sur des charges en suspensions mais toujours sur des débits solides $Q_{\mathrm{s}}$ en $\mathrm{kg} / \mathrm{s}$, tels que $Q_{\mathrm{s}}=Q_{1} \cdot C_{\mathrm{s}}$.

En raison de la variabilité de la fonction $Q_{s}=f\left(Q_{1}\right)$, il faut déterminer pour chaque rivière un intervalle de temps pendant lequel on considère que 1a charge en suspension ne sera pas trop variable et auquel on pourra donc attribuer une charge en suspension déterminée. Cet intervalle peut aller de quelques heures (cas de la Dranse) à plusieurs jours (cas des rivières à crue annuelle régulière). Pour le Rhône nous avons pris une journée, cet intervalle est sans doute encore trop grand, la turbidité pouvant en effet encore varier beaucoup. Si l'on dispose d'une mesure $\left(\mathrm{C}_{\mathrm{si}}\right)$ par intervalle de temps choisi, on la considère comme représentative et on peut effectuer le calcul $Q_{s i}=Q_{1 i} \cdot C_{s i}$. La masse des suspensions transportée pendant la période $T$ sera alors d'après les $\mathrm{N}$ mesures :

$$
M_{S}=\frac{T}{N} \sum_{i=i}^{i=N} Q_{S i}
$$

Si l'on ne dispose pas d'une mesure pour chaque intervalle de temps choisi, deux solutions sont possibles

- soient $\mathrm{n}$ mesures de $\mathrm{C}_{\mathrm{sj}}$ pendant la période $\mathrm{T}$ on calculera le débit solide moyen

$$
Q^{\prime}{ }_{s}=\sum_{j=i}^{j=n} \frac{Q_{s j}}{n} \text { puis la masse totale } M^{\prime}{ }_{S}=Q^{\prime}{ }_{s} T
$$

Mais par cette méthode on risque de laisser passer entre deux mesures une crue dont $1^{\prime}$ effet peut être très important. Ceci est facilement vérifiable si on considère les débits liquides. Il faut donc extrapoler entre deux mesures de turbidité.

Nous sommes alors conduits à attribuer à notre intervalle de temps une valeur de turbidité en se basant sur le débit, la position de 1'intervalle par rapport au cycle hydrologique, etc..., ce qui est possible si on a pu tirer de la série incomplète de mesures, des lois de variations de la turbidité valables pour l'ensemble de la période $\mathrm{T}$.

Pour notre part nous avons essayé de faire le bilan des apports en suspension du Rhône en 1967. Nous avons d'abord déterminé les lois de variations de la turbidité en fonction du débit de crues pour deux périodes : hautes eaux d'été et basses eaux d'hiver. Puis nous avons affiné ces relations en séparant encore les montées des eaux des décrues. Nous avons alors attribué à chaque journée sans mesure, une valeur de turbidité basée sur les relations précédentes, en tenant compte des valeurs de turbidité mesurées encadrant notre journée. 
Nous avons ensuite calculé les débits solides journaliers avec les valeurs de turbidités -mesurées et ainsi extrapolées- et les débits liquides journaliers. Cette méthode est bien sûr encore trop approchée puisqu'on devrait tenir compte des débits instantanés mais il nous semble qu'elle tient déjà compte des paramètres qu'on peut étudier. Comme nous 1'avons dit, la turbidité dépend encore d'autres facteurs beaucoup plus difficilement analysables, il faut donc arriver à mesurer la turbidité en continu.

\section{2) Les mesures en contînu de la turbidité.}

Elles suppriment les inconvénients et les incertitudes des calculs de bilan. Néanmoìns, ces méthodes sont souvent incomplètes : les appareils de mesure en continu sont en général fixes, on doit donc par de nombreuses comparaisons avec la méthode par pesée, déterminer le rapport entre la turbidité mesurée par 1'appareîl et la turbidité réelle de toute la section. Le débit liquide $Q_{1}(t)$ étant également enregistré, on peut effectuer le calcul du débit solide instantané $Q_{s}(t)=Q_{1}(t) \cdot C_{s}(t)$.

3) I1 est important de connâ̂tre la densité des a1luvions transportées : cette densité varie généralement de 1,5 pour les sédiments humides à 2,7 pour les sédiments secs, il est donc nécessair de donner la nature des alluvions transportées. On peut ainsi déterminer le volume des apports et par là même arriver à la notion d'érosion spécifique, exprimée en $t / \mathrm{km}^{2} / \mathrm{an}$ ou en $\mathrm{mm} / 1000$ ans, ce qui est très utile en géomorphologie.

\subsection{2. - Apports du Rhône.}

Nous avons effectué une étude qualitative d'après les données de 1'Office Fédéral de 1'Economie Hydraulique de Berne. Nous verrons que pour les études quantitatives les résultats obtenus sont faibles, même si on considère les faibles débits de l'année considérée. Nous nous sommes alors basés sur les estimations de 1'EPUL (dans Houbo1t, 1968).
4.1.2.1. - Variations saisonnières et interannuelles.

Nous avons portê sur la figure $\mathrm{IV}_{1}$ la relation entre la charge en suspension $\mathrm{Cs}$ en $\mathrm{g} / \mathrm{m}^{3}$-généralement mesurée vers midi- et le débit liquide moyen en $\mathrm{m}^{3} / \mathrm{s}$ du même jour pour trois ans. La relation est de la forme $\mathrm{Cs}=\mathrm{AQ}_{1}{ }^{\mathrm{B}}$, cette 1 oì est générale pour tous les transports en suspension quelque soit le climat (voir Müller, 1968). Si on prend soin de séparer les mesures effectuées en hautes eaux d'été de Mai à Septembre et celles effectuées en basses eaux d'hiver d'Octobre à Avril, deux corrélations différentes sont nettement visibles.

Dans la relation $\mathrm{Cs}=\mathrm{A} Q_{1}{ }^{\mathrm{B}}$, le facteur $\mathrm{A}$ rend compte de la turbidité générale de la rivière, le facteur $B$ de la variabilitê de la turbidité en fonction du débit. Les coefficients numériques $A$ et $B$ dépendront donc de 1 a saison. On peut voir sur la figure $I V_{1}$ que les turbidités sont plus fortes, à débit égat, en hiver qu'en été. Cela est facilement explicable : la turbidité est surtout liée à des variations de débits, en hiver les débits de l'ordre de $200 \mathrm{~m}^{3} / \mathrm{s}$ correspondront aux crues, en été par contre ces débits correspondront aux étiages relatifs sans érosion importante. De plus on note que la turbidité augmente plus vite en fonction du débit en hiver qu'en été : les eaux en hiver ont donc un pouvoir d'érosion plus important, le ruissellement dû aux averses et aux orages d'automne ou à la fonte des neiges de basses altitudes,est sans doute supérieur à celui provoqué en été par la fonte des glaciers.

Si la variation saisonnière est primordiale il y a également des variations interannuelles; ainsi sur la figure $\mathrm{IV}_{2}$ on voit bien la différence en hiver comme en été, entre l'année 1965 et l'année 1966. I1 y a un décalage des nuages de points, 1'année 1966 ayant des turbidités plus faibles, à débit égal que 1'année 1965.

Nous pouvons comparer le comportement du Rhône à 1a Porte du Scex et du Rhin à Lustenau grâce aux travaux de Müller (1968). 


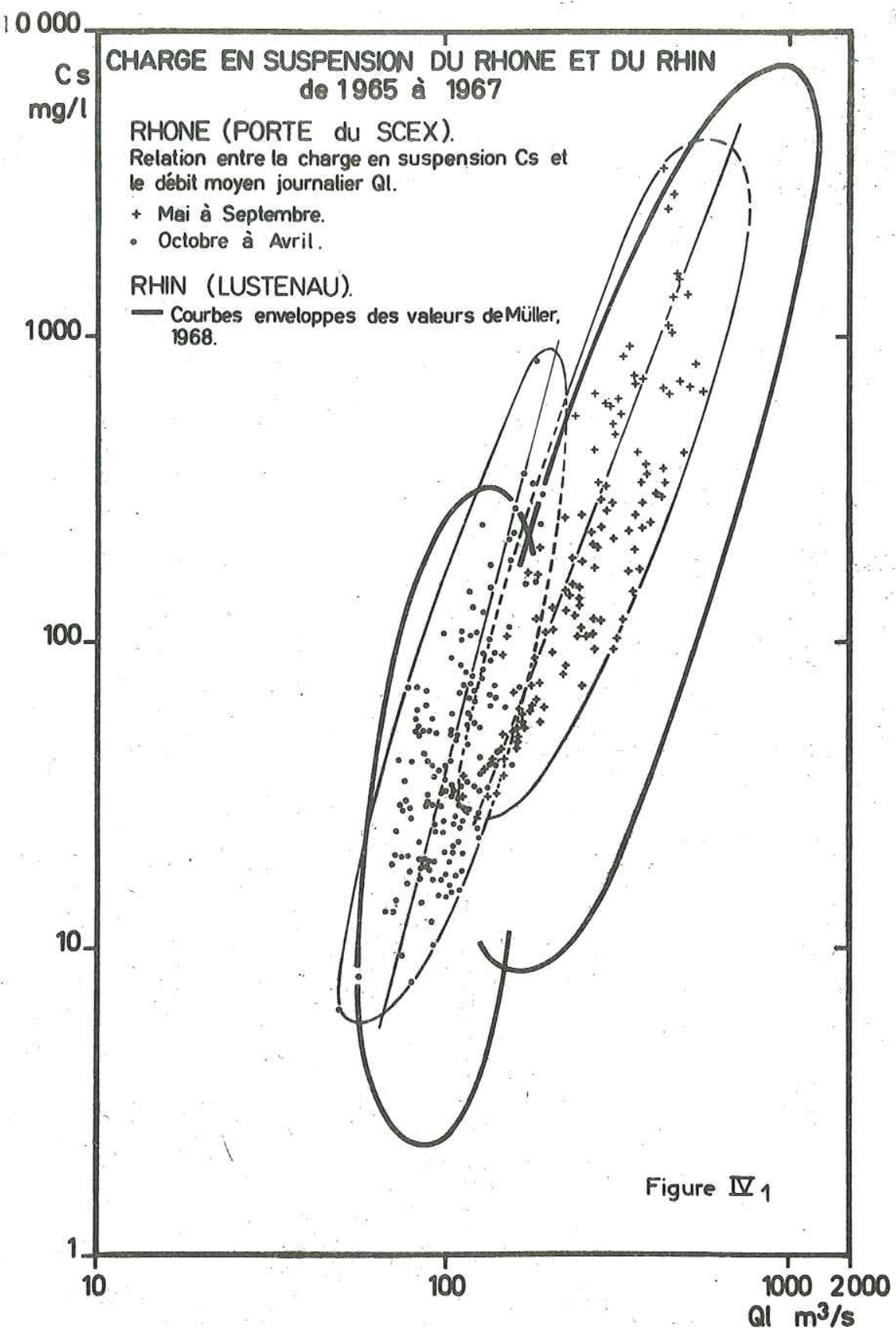

La figure $\mathrm{IV}_{1}$, montre les courbes enveloppes des points représentant $C_{s}=f\left(Q_{1}\right)$ pour les mêmes années 1965 à 1967. Dans son article Müller n'avait pas séparé les valeurs obtenues en période de basses eaux et de hautes eaux mais sur sa figure îl y a très nettement deux corrélations différentes, nous avons matérialisé ces deux nuages de points. Les remarques sur l'érosion suivant la saison sont donc également valables pour le Rhin. Nous voyons que les deux rivières ont pour les deux périodes hydrologiques considérées des relations presque identiques : une fois de plus la similitude des bassins versants, des régimes et des terrains aboutit à des valeurs semblables.

\subsubsection{2. - Variation de la charge solide au sein}

d'une crue.

Comme nous $1^{\prime}$ avons dit, du fait du décalage entre $1 \mathrm{e}$ maximum de charge en suspension et le maximum de débìt liquide, les turbidités en montée des eaux sont toujours plus fortes que celles en décrue. Ce phénomène est général et a été remarqué sur de très nombreuses rivières. La figure $\mathrm{IV}_{3}$ montre bien sur trois années des corrélations nettement différentes $\mathrm{C}_{\mathrm{S}}=\mathrm{A}_{\mathrm{Q}}{ }^{\mathrm{B}}$ en crue et en décrue.

4.1.2.3. - Calcul des apports du Rhône en suspensions pour 1967.

Choisissant 1'année 1967 nous avons donc procédé comme suit :

- Etablissement des lois de variations $\mathrm{C}_{\mathrm{S}}=\mathrm{A} \mathrm{Q}_{1}{ }^{\mathrm{B}}$ pour $1^{\text {'an- }}$ née considérée en séparant les périodes de régimes hydrologiques différents et les valeurs obtenues en crue ou en décrue, soit quatre relations basées sur 103 mesures de 1'Office Fédéral de 1'Economie Hydraulique.

- Interpolation des valeurs de $\mathrm{C}_{\mathrm{s}}$ pour les jours sans 

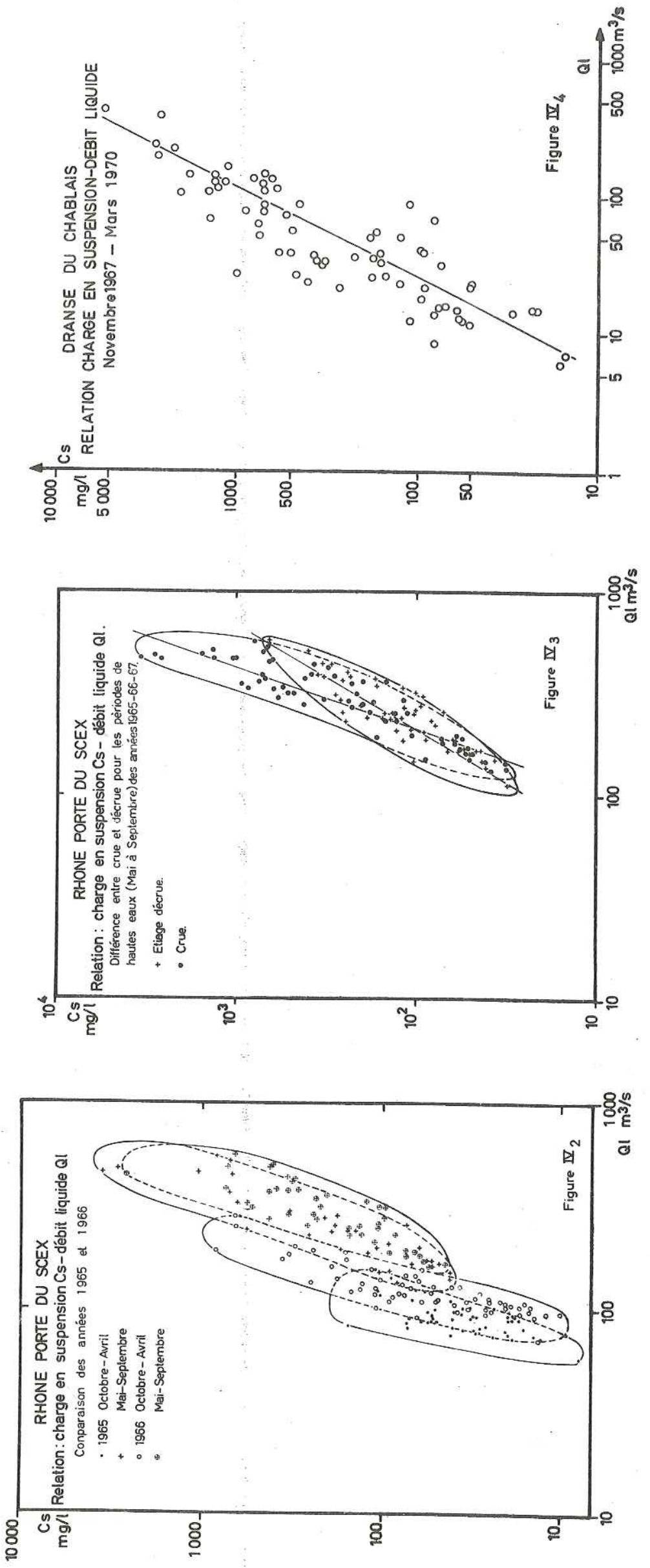

mesures en tenant compte des lois précédemment établies et des valeurs mesurées de part et d'autre.

- Intégration de 1 a fonction $Q_{s}(t)=C_{s}\left(t, Q_{1}\right) \cdot Q_{1}(t)$ sur toute l'année avec un pas d'intégration d'une journée

$$
M_{S}=\int_{0}^{T} Q_{S}(t) d t
$$

On arrive ainsi à un dêbit solide moyen annuel de $Q_{s}=45 \mathrm{~kg} / \mathrm{s}$ ce qui correspond à $1,4210^{6} \mathrm{t}$ de matières en suspension transportées au Léman pendant 1'année 1967.

Nous avons porté sur le tableau IV 1 les débits liquides de 1967 comparés aux débits 1iquides interannuels de 1935 à 1967, puis le détail du calcul du débit solide moyen mensuel et de la turbidité par les trois méthodes suivantes :

1) Méthode des moyennes des débits solides.

Débit solide moyen mensuel $Q_{\mathrm{sm} 1}=\sum_{i=1}^{i=30} Q_{1 i} \cdot C_{\mathrm{si}} / 30$

avec $\mathrm{C}_{s i}$ charge solide (mesurée et extrapolée) du ième jour

$Q_{1 i}$ débit liquide moyen du ième jour.

Masse annuel1e de suspension $M_{s a 1}=T \sum_{j=1}^{j=12} Q$ smj/12

avec $Q_{1 \mathrm{mj}}$ débit liquide moyen mensuel du j $j^{\text {ième }}$ mois V1a volume liquide annuel

T durée d'une année 
Charge solide moyenne mensuelle $C_{\text {smj }}=Q_{s m j} / Q_{1 \mathrm{mj}}$

Charge solide moyenne annuelle $\mathrm{C}_{\mathrm{sal}}=\mathrm{M}_{\mathrm{sal}} / \mathrm{Vla}=\mathrm{Q}_{\mathrm{sal}} / \mathrm{Q} 1 \mathrm{a}$

2) Moyenne des charges solides (mesurées et interpolées).

$$
\begin{aligned}
& C_{s m 2}=\sum_{i=i}^{i=30} C_{s i} / 30 \\
& Q_{s m 2}=Q_{1 m} \cdot C_{s m 2} \\
& M_{s a 2}=T \cdot \sum_{j=i}^{j=12} Q_{s m j} / 12
\end{aligned}
$$

3) Moyenne des charges solides mesurées.

$$
\begin{aligned}
& C_{s m 3}=\sum_{i=1}^{i=n} C_{s i} / n \quad a v \\
& Q_{s m 3}=Q_{1 m} \cdot C_{s m 3} \\
& M_{s a 3}=T \sum_{j=i}^{j=12} Q_{s m j} / 12
\end{aligned}
$$$$
\text { avec } \mathrm{n} \text { mesures dans un mois }
$$$$
\mathrm{C}_{\text {si }} \text { charge solide mesurée du } i^{\text {ième }} \text { jour }
$$

Le tableau $\mathrm{IV}_{1}$ montre bien les grandes différences obtenues uniquement en changeant les méthodes de calculs. On voit aussi que les façons de calculer la moyenne annuelle de turbidité sont multiples. Comme pour les charges en éléments dissous $C_{d}$ il convient de différencier les concentrations les plus fréquentes, les moyennes arithmétiques et les moyennes pondérées par les débits, ces dernières intervenant seules dans les bilans. Ainsi à côté des turbidités moyennes annuelles $C_{\text {sa }}$ calculées en divisant la masse annuelle de suspension par le volume liquide annuel, on peut ainsi calculer pour chaque méthode les moyennes arithmétiques des charges en suspensions mensuelles. On aura alors :

$$
\mathrm{C}_{\mathrm{s}^{\prime} \mathrm{a}}=\mathrm{C}_{\mathrm{sm}} / 12
$$

En raison du type de loi les moyennes arithmétiques sont systématiquement inférieures aux moyennes pondérées :

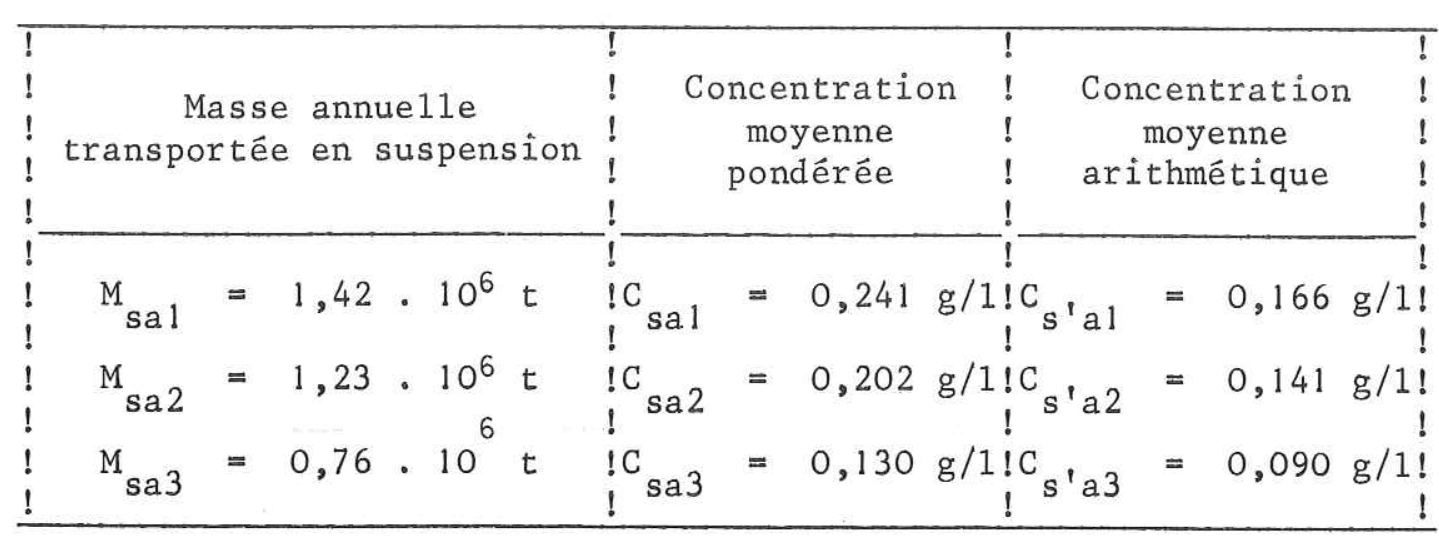

\subsubsection{4. - Apports solides du Rhône au Léman.}

La valeur obtenue pour 1967 est sans doute très sousestimée pour deux raisons : d'abord les prélèvements sont effectués vers le milieu de la journée, or ce moment correspond à un minimum journalier de débit en raison du régime glaciaire du Rhône en été ; de plus 1'année 1967, bien qu'ayant un débit liquide moyen annuel se rapprochant de très près du module interannuel, n'a eu que très peu de fortes crues par rapport aux années normales (on note juste une crue en Février, responsable d'ailleurs de la forte turbidité relative de ce mois). Il convient donc de se reporter à d'autres estimations. Les études du laboratoire d'Hydraulique de 1'EPUL à Lausanne (d'après Houbolt, 1968 et Vernet, 1969) arrivent aux conclusions suivantes

- années sèches 3 - 4 millions de tonnes/an (année 1949)

- années humides 9 -10 millions de tonnes/an (année 1945)

- années moyennes 6- 7 millions de tonnes/an (période 1935 - 1962). 
Pour ce qui est des matières apportées au lac par charriage de fond elles sont estimées à 0,1 million de tonnes. Ce très faible apport, de 1 'ordre de $1 \%$ des apports en suspensions, est analogue aux estimations de Müller pour 1e Rhin. Ce chiffre peut sembler faible -on estime en général cette proportion à $10 \%$, et elle peut aller jusqu'à $50 \%$ pour certains torrents alpins-mais il ne faut pas oublier que le Rhin et le Rhône peuvent deposer dans leurs plaines alluviales 1a plupart de leurs êléments grossiers.

Nous avons reporté sur le tableau $\mathrm{IV}_{2}$ les érosions spécifiques détritiques calculées d'après les nombreuses données bibliographiques (Collet, Pardé, etc...). Les valeurs sont assez différentes : notre estimation pour 1967 est la plus faible pour les raisons que nous avons évoquées, celle de l'EPUL est la plus élevée. Pour la comparaison des érosions spécifiques nous choisirons cependant cette dernière valeur qui correspond d'ailleurs à l'estimation des apports du Rhône (voir chapitre V) depuis la fin des glaciations wurmiennes : 8 millions de tonnes par an pendant 15000 ans.

\subsection{3. - Apports de la Dranse.}

Nous ne disposons malheureusement pas de données aussi complètes sur 1a Dranse que sur 1e Rhône. Nous avons porté sur 1a figure $I_{4}$ les résultats des premières mesures effectuées depuis 1967. Comme pour le Rhône la relation générale $\mathrm{Cs}=\mathrm{A} Q_{1}^{B}$ est bien nette, mais nous ne pouvons pas encore différencier les relation spécifiques pour les crues et décrues, ni effectuer un bilan sur ces premiers résultats. Aussi nous avons évalué la valeur du delta de 1a Dranse dans 1e Léman à $1,5 \mathrm{~km}^{3}$ soit $4.10^{9}$ tonnes en admettant que ce delta s'est construit depuis le dernier retrait des glaciers il y a 15000 ans on aurait alors un dépôt annuel de 260000 t/an de graviers, sables et galets. Cet apport représente à la fois le charriage de fond de la Dranse et une partie des alluvions transportées en suspensions. Compte tenu des alluvions en suspensions plus fines sédimentées plus loin nous pensons que l'apport annuel moyen doit être de 500000 à 1 million de tonnes par an, ce qui correspondrait à une érosion spéciffique à 1000 à 2000 $\mathrm{t} / \mathrm{an} / \mathrm{km}^{2}$. $=1 \mathrm{~kg} / \mathrm{m}^{2}$. \#2,5 mm d'c jeurnem de terre.

\subsection{4. - Effets des crues}

Les crues ont une action déterminante en sédimentologie : dans nos régions les apports de quelques jours de crue peuvent atteindre 1'ordre de grandeur des apports annuels. Une étude détaillée de 1a crue du 22 Septembre 1968 de 1a Dranse du Chablais a pu être menée tant sur le plan hydrologique, hydrochimique que sédimentologique (voir Hubert et al, 1969). Nous en tirons 1a comparaison suivante :

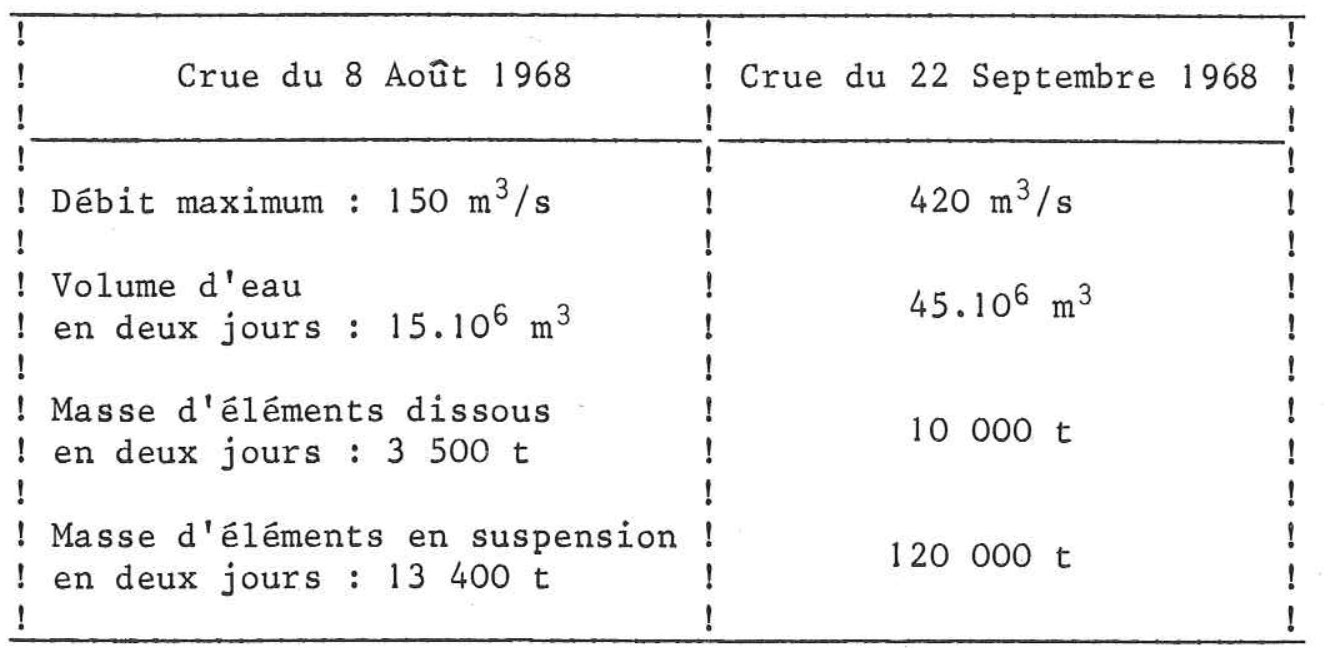

Si le volume est multiplié par 3 dans la crue du 22 Septembre, les apports en suspension sont multipliés par 9, on mesure encore plus 1'importance d'une telle crue lorsqu'on remarque qu'elle a apporté en deux jours de 20 à $30 \%$ des apports annuels. 
A limnologist is a zoologist who, during the summertime, studies chemical and botanical aspects of geological problems in readily accessible lakes, $15 \mathrm{~m}$ deep, located in the vicinity of universities.

J.R. Vallentyne

Limnology et Oceanography Septembre 1969
Il en est de même pour le Rhône et le Rhin. Nous avons calculé, d'après les relations $C_{s}=f\left(Q_{1}\right)$ pour les montées des eaux, les débits solides de quelques crues du Rhône que nous comparons aux crues analogues du Rhin (d'après Mü11er, 1966). Il est à remarquer de nouveau qu'une forte crue d'hiver a un débit solide bien plus élevé qu'une crue d'été de même débit liquide.

\begin{tabular}{|c|c|c|c|c|c|c|}
\hline$!$ & $\begin{array}{l}! \\
!\end{array}$ & \multicolumn{2}{|c|}{ Rhône } & \multicolumn{3}{|c|}{ Rhin } \\
\hline $\begin{array}{l}\vdots \\
\vdots \\
\vdots \\
\vdots\end{array}$ & $\begin{array}{l}\vdots \\
\vdots \\
\vdots \\
\vdots\end{array}$ & $\begin{array}{c}\text { Débit } \\
\text { liquide } \\
\text { ournalier } \\
\mathrm{m}^{3} / \mathrm{s}\end{array}$ & $\begin{array}{l}! \\
! \text { Débit } \\
! \text { solide } \\
! \mathrm{t} / \mathrm{j} \\
!\end{array}$ & $\begin{array}{c}\text { Débitt } \\
\text { liquide } \\
\text { journalier } \\
\mathrm{m}^{3} / \mathrm{s} \\
\end{array}$ & \begin{tabular}{|c}
$!$ \\
$\vdots$ \\
$\vdots$ \\
solidite \\
$t$ \\
$t$
\end{tabular} & te \\
\hline !Crue ordinaire d'été & $!$ & 500 & $75000 !$ & 400 à $700 !$ & 28 & $000 !$ \\
\hline !rès forte crue d'été & $!$ & 850 & ! $750000 !$ & $!$ & $950 \mathrm{c}$ & $000 !$ \\
\hline $\begin{array}{l}\text { Crue exceptionnelle d'été } \\
\vdots\end{array}$ & $\vdots$ & & $\begin{array}{ll}! & ! \\
! & \vdots\end{array}$ & $\begin{array}{l}2500 \text { à } 2700 ! \\
22 \text { Août } 1954 !\end{array}$ & $!$ & $000 !$ \\
\hline ! Crue ordinaire d'hiver & $!$ & 130 & ! $2000 !$ & & $!$ & \\
\hline !rès forte crue d'hiver & $!$ & 300 & $! 250000 !$ & & & $!$ \\
\hline
\end{tabular}

Les débits solides annuels peuvent ainsi facilement varier de 1 à 10 : pour le Rhin Müller (1966) cite un volume de sédiment transporté de $560000 \mathrm{~m}^{3}$ en 1949 et de $5070000 \mathrm{~m}^{3}$ en 1935. Aussi les mesures de turbidités doivent-elles être poursuivies sur des dizaines d'années pour avoir des valeurs moyennes correctes, nous y reviendrons.

\subsection{5. - Comblement du Léman.}

$\mathrm{Si}$ on considère un apport de 6 à $7.10^{6} \mathrm{t} / \mathrm{an}$ pour le Rhône, un apport de 0,5 à $1,0 \cdot 10^{6} \mathrm{t} /$ an pour la Dranse et autant pour le reste des affluents, on arrive à une valeur comprise entre 7 et 9 millions de t/an de matériaux secs. On aura donc un taux de 
sédimentation annuelle moyenne sur tout le lac de $1,40 \mathrm{~g} / \mathrm{cm}^{2} / a n$. I1 va sans dire que cette vitesse est très variable et que la grosse majorité des apports détritiques au Léman sert à accroître les deltas du Rhône, de la Dranse et des autres petîtes rivières. I1 est probable qu'au centre du Léman les valeurs des vitesses de sédimentation soient considérablement plus faỉbles, nous verrons plus loin l'ordre de grandeur de la sédimentation annuelle dans la plaine centrale. et chimiques.

\subsection{6. - Comparaison des érosions spécifiques détritiques}

\subsubsection{1. - Taux d'érosions actuels des Alpes centrales.}

Nous avons reporté dans le tableau $\mathrm{IV}_{2}$ les érosions spécifiques calculées d'après les nombreuses données bîblìographiques. Les érosions chimiques sont assez fortes et comprises entre

$165 \mathrm{t} / \mathrm{km}^{2} /$ an pour 1e Pô et $535 \mathrm{t} / \mathrm{km}^{2} /$ an pour 1a Bregenzer Ach. Pour 1'Arve nous avons augmenté la valeur donnée par Baeff(Forel Le Léman, 1890), qui nous semblait beaucoup trop faîble vu les analyses modernes et la valeur de la Dranse : en reprenant les valeurs journalières de Baeff et en appliquant la méthode d'analyse décrite au chapitre II, nous trouvons une concentration globale moyenne de $200 \mathrm{mg} / 1$.

I1 est à remarquer que pour le Rhône et le Rhin, l'érosion spécifique chimique varie peu entre l'entrée et la sortie des lacs Léman et de Constance. Il n'en est bien sûr pas de même pour les érosions détritiques : cette différence entre les deux types d'érosion est très importante, elle s'ajoute à celle que nous avons déjà vue pour les crues. (La valeur moyenne de $20 \mathrm{mg} / 1$ de turbidité pour 1e Rhin lacustre, d'après Müller 1966, que nous avons choisie nous semble assez forte comparée au Rhône lacustre pour lequel nous n'avons trouvé que $4,0 \mathrm{mg} / 1$ en moyenne sur 3 ans d'après les données de 1'Office Fédéral de 1'Economie Hydraulique).

Pour les érosions détritiques nous remarquons que les valeurs du versant nord des Alpes sont en général plus fortes que ce1les du versant sud, seule la Dora Baltea a des érosions spécifiques analogues, 1'Adige est beaucoup plus faible (nous avons bien sûr éliminé de ce tableau les rivières sortant des grands lac italiens).

En considérant les bassins versants de la Dranse du Chablais, du Rhône alpestre, du Rhin alpestre, de la Bregenzer Ach, de 1'Arve et de la Dora Baltea, soit un total de $18000 \mathrm{~km}^{2}$ on arrive à une érosion détritique moyenne de $900 \mathrm{t} / \mathrm{km}^{2} / \mathrm{an}$. Ces chiffres sont encore très imprécis, surtout pour les érosions détrîtiques qui sont quelquefois à $50 \%$ près, néanmoins nous pouvons compter sur un ordre de grandeur d'érosion totale de 1200 t/an compte tenu également des transports sur le fond.

Pour la région centrale alpine l'érosion est alors de $0,45 \mathrm{~mm} / \mathrm{an}$ ou de $450 \mathrm{~mm} / 1000$ ans. Cette valeur correspond normalement aux érosions des reliefs accusés sous des climats à régime pluvial continental. Ainsi dans son excellent article Young (1969) donne des valeurs allant de 92 à $970 \mathrm{~mm} / 1000$ ans pour les reliefs accusés.

\subsubsection{2. - Comparaison des érosions chimiques et}

\section{détritiques.}

A 1a suite de Judson et Ritter (1964) et Durum, Heidel et Tison (1960), nous avons essayé d'étudier les variations comparées des érosions chimiques et détritiques. Pour les premiers auteurs, qui se basent sur des valeurs pour les Etats-Unis, 1'érosion spécifique détritique diminue quand l'érosion spécifique chimique augmente. Nous avons reporté sur la figure $\mathrm{IV}_{5}$ les valeurs des érosions obtenues pour les Alpes, celles de Judson et Ritter pour les U.S.A. 
et quelques autres : rivière Mc Kenzie au Canada (Levinson et al, 1969), 1'Amazone et le Congo (Gibbs, 1967). Il semble bien que les érosions augmentent en même temps : les valeurs du Colorado et de la cote pacifique ont poussé Judson et Ritter à affirmer 1'inverse. En fait il semble que pour ces régîons, surtout pour le Colorado les érosions détritiques sont tout à fait à part en raison du climat très différent de celui des autres rivières. En général, les érosions semblent d'abord être liées à la taîle du bassin-versant puis au climat. Comme la concentration en élements dissous varie peu au long d'une rivière, l'érosion spécífique chimique tend à diminuer vers 1'embouchure; de même 1 'érosion détritique baisse en raison du relief peu accusé et de la vitesse plus faible des courants.

Pour Durum, Heidel et Tison, la concentration en élément dissous croît si le débit spécifique lîquide diminue. Là aussi ces auteurs ont tiré des conclusions pour les Etats-Unis, il ne semble pas que cette loi de variation s'applique aux rivières alpines, comme le montre la figure $\mathrm{IV}_{6}$ sur laquelle nous avons reporté les couples de valeurs pour 18 rivières du monde entier (d'après les

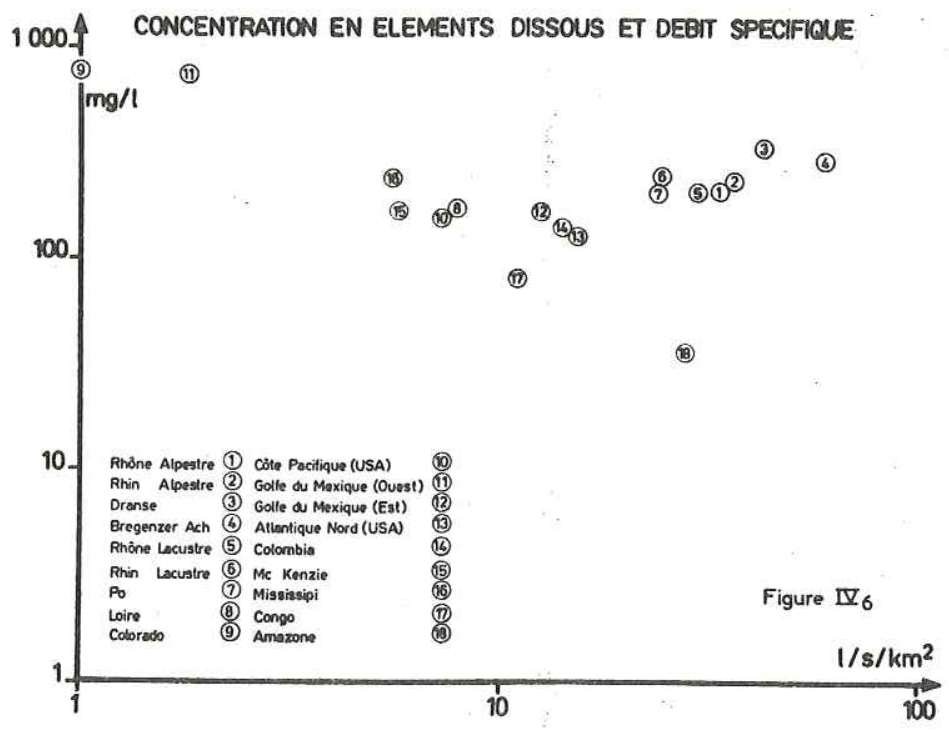
mêmes auteurs que précédemment, voir tableau $\mathrm{IV}_{2}$ ).

Ces deux études comparatives montrent bien que notre connaissance des phénomènes d'érosions est encore lìmitée, malgré des études nombreuses et poussées surtout pour les érosions détritiques (Fournier, 1960). Seule la comparaison d'un grand nombre de rivières à caractères différents (climat, végétation, relief, type de roches, etc...) permettra de détérminer 1 'importance relative de chaque paramètre.

4.1.6.3. - Valeur des érosions mesurées actuellement.

Nous avons déjà vu que pour une même rivière les érosions chimiques sont relativement constantes (variation du simple au double) tandis que les érosions détritiques sont soumises à des 
variations très importantes : augmentation très forte lors des crues ou diminution complète par l'effet de retention des bassins. Il en résulte que sí l'on peut avoir une bonne approximation des érosions chimiques, l'estimation des érosions détrîtiques est beaucoup plus délicate et nécessite des dizaines d'années de mesures. Néanmoins il est très probable que même pendant ce laps de temps important à 1 'échelle humaine, des phénomènes courants à 1 'échelle géologique comme les grands séismes, les crues centenaîres et millểnaìres, les grands glissements de terrains, etc... ne vont pas se produire. L'action de ces phénomènes exceptionnels est en fait aussi importante pour la dégradation et le transport que la lente érosion annuelle.

Un exemple de phénomènes exceptionnels nous est donné dans le bassin du Léman : en 1 'an 563 après Jésus-Christ, il s'est produit dans la vallée du Rhône un formídable éboulement à Tauredunum qui a formé un lac en barrant la vallée. Le barrage s'est rompu brutalement provoquant une "crue" du Léman qui eu des effets catastrophiques. Le souvenir de cet événement rapporté entre autres par Grégoire de Tours s'est conservé pendant tout le moyen-âge (Forel, Le Léman, tome III, p 496). Il est donc difficile de mesurer à notre échelle de temps un tel phênomène qui est à 1'échelle géologique, ir semble préférable d'effectuer des estimations indirectes de l'érosion détritique notamment en considérant non pas l'oblitération des reliefs mais le remplissage des bassins (Tricart, 1962). On intègre ainsi les phénomènes exceptionnels à notre échelle. Un exemple sera donné au chapitre $V$ pour le remplissage de la cuvette lémanique. A une autre échelle citons le remplissage du bassin du Pô au quaternaire : Gabert (1960) y estime en volume des sédiments quaternaires à $40000 \mathrm{~km}^{3}$, ce qui correspond à une érosion spécifique de $1250 \mathrm{t} / \mathrm{km}^{2} /$ an en considérant une durée de 500000 ans pour le quaternaire ou bien $625 \mathrm{t} / \mathrm{km}^{2} /$ an pour une époque quaternaire d'un mi1lín d'années.

\section{2. - Nature physique des apports.}

\subsection{1. - Mode de prélèvement et méthodes d'analyses des} sédiments.

Les sédiments du Rhône et de la Dranse ont été prélevés à la main sur les berges. Les eaux turbides ont été prises généralement en bidons de 301 , quelquefois la faible turbidité de la rivière nous a conduit à prendre plus de 200 litres. Les prises de suspensions ont essentiellement servi aux analyses chimiques, aucune détermination des caractéristiques physiques n'étant possible sur d'aussi faibles quantités (quelques grammes le plus souvent).

Sur les sédiments ont été réalisés systématiquement les séparations de la fraction fine de la fraction grossière par tamisage sur tamis de 40 microns et quand il y avait lieu des granulométries de fraction grossière, non attaquée à l'acide sur colonne de tamis agités par un système ROTAP pendant 20 minutes. Les calcimétries des fractions fines ont été faites au calcimètre Bernard dont la précision absolue moyenne est de $0,5 \%$ de $\mathrm{CaCO}_{3}$. Pour la fraction grossière le pourcentage de carbonate a été déterminé par différence de pesée après attaque à 1'acide chlorhydrique. Les teneurs en eau sont données par rapport au poids de la vase sèche. Les densités ont été réalisées au picnomètre.

\subsection{2. - Les apports du Rhône.}

\section{Granulométrie.}

De nombreuses déterminations ont été réalisées à 1 'Ecole Polytechnique de Lausanne, sur la base de ces analyses Houbolt et Jonker (1968) et Vernet (1969) citent les rêsultats suivants : 


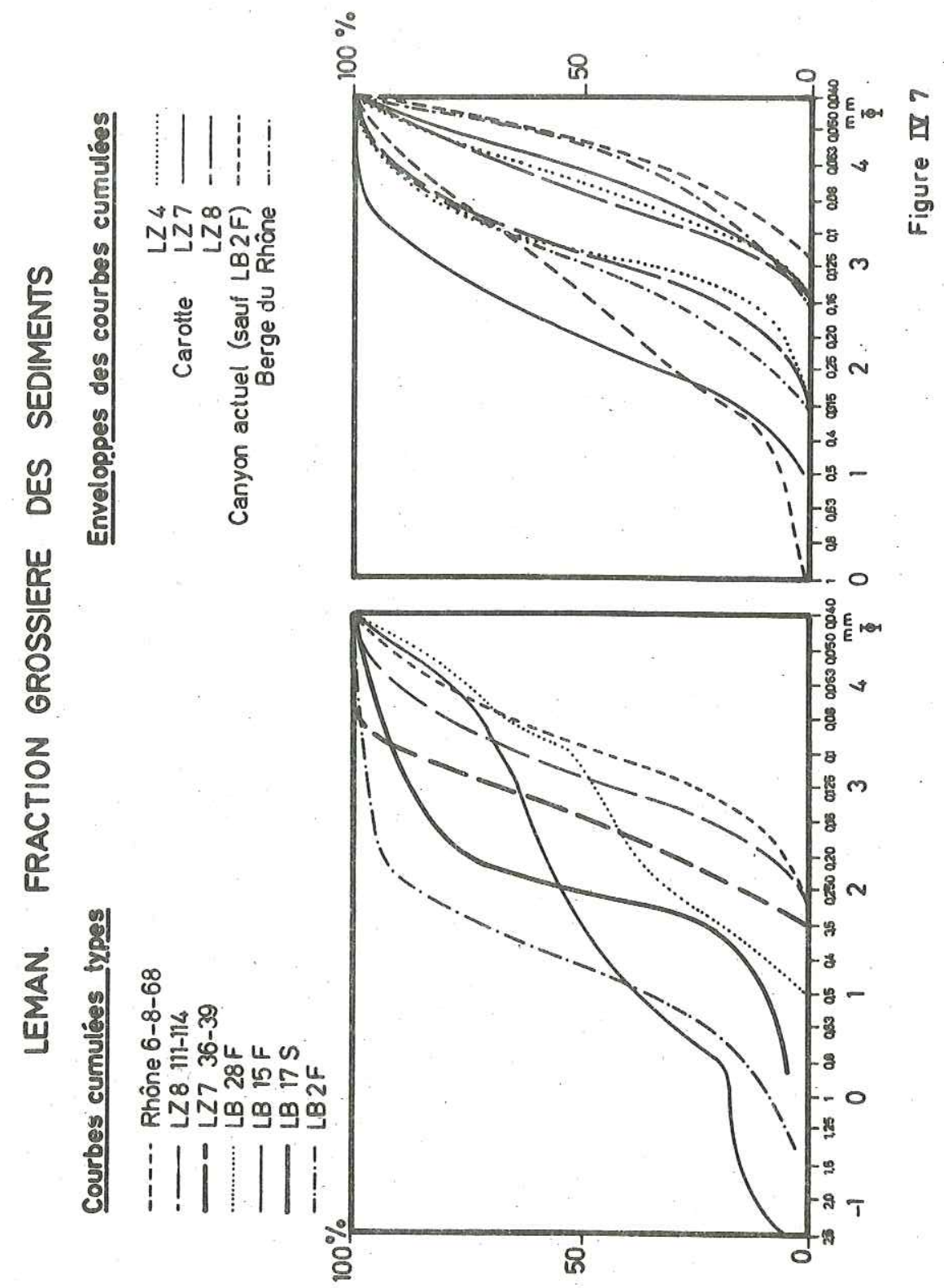

Vernet

$\begin{array}{ccc}60-250 \mu & 20-85 \% \\ 20-60 \mu & 15-80 \% \\ <2 \mu & \text { absent }\end{array}$

sables grossiers maximum de $35 \%$

$$
\begin{array}{lr}
\text { sable fin } & 20-85 \% \\
\text { limon } & 18-80 \% \\
\text { argile } & 0-5 \%
\end{array}
$$

I1 ressort de ces résultats que le Rhône charrie très peu d'argiles et de sables grossiers, la majeure partie des apports est constituée de sables fins et de sablons $(100 \mu$ à $20 \mu)$ ou par des silts $(63 \mu-4 \mu)$. Nos résultats (tableau $\mathrm{IV}_{3}$ en annexe) confirment ces données, 1a fraction grossière (> $40 \mu$ ) varie de $47 \%$ à $92 \%$ et 1a médiane des courbes cumulées de $76 \mu$ à $115 \mu$, le sédiment grossier est toujours bien classé.

Nous donnons sur la figure $\mathrm{IV}_{7}$ une courbe cumulée typique de la fraction grossière ainsi que l'enveloppe d'une dizaine de courbes cumulées. La granulométrie de la fraction fine n'a pas été réalisée, citons toutefois une détermination réalisée avec un appareil Coultronics (compteur Coulter) sur le prêlèvement du 16 Octobre 1968, de très faible turbidité, recueilli sur filtre : la médiane est de $6 \mu$.

\section{Densités et calcimétries.}

Les résultats sont portés dans le tableau $\mathrm{IV}_{3}$. Vu le mode de prélèvement aucune mesure de densité humide n'a été réalisée, la densité sèche est de 2,55 à 2,7 . La teneur en $\mathrm{CaCO}_{3}$ varie de 16 à $29 \%$ pour la fraction fine et de 15 à $34 \%$ pour la fraction grossière sans qu'il soit possible de mettre en évidence des variations saisonnières.

Nature minéralogique des apports.

Outre les éléments légers très abondants quartz, micas, 
feldspaths, on trouve parmì les minéraux lourds surtout de 1'épidote, de 1'amphibole puis le grenat, zircon, et dans une moindre mesure apatite, g1aucophane, etc... Ces déterminations font partie d'une étude plus générale de S. Duplaix du Laboratoire de Géologie Dynamique, en cours depuis plusìeurs années avec le C.R.G. de Thonon. Pour ce qui est de 1 a fraction argileuse Vernet (1969) a analysé les échantillons recueillis par l'EPUL et donne la composition suivante (fraction $<7 \mu$ ).

$$
\begin{array}{ll}
\text { illite } & 40 \text { à } 50 \% \\
\text { chlorite } & 20 \text { à } 30 \% \\
\text { kaolinite } & 20 \text { à } 30 \%
\end{array}
$$

La présence d'illite et de chlorite est bien confirmée par une analyse effectuée par diffractométrie, A.T.D. et par diagramme de Debye-Scherrer au laboratoire d'analyse des argỉles (B. Siffert) de 1'E.S.C.M. de Mulhouse, par contre la kaolinite n'avait pas été détectée en aussi grande quantité.

\subsection{3. - Dranse.}

La détermination de 1a qualité des apports de 1a Dranse est beaucoup plus dêlicate en raison de l'hétêrogénéité très grande de ceux-ci allant des blocs jusqu'aux argiles. Le prélèvement de sédiment est donc dêlîcat et les résultats correspondant ne peuvent être qu'indicatífs. Les galets sont bien sûr essentiellement calcaires (calcaires compacts, dolomitiques, bréchíques, etc...) mais la destruction des dépôts fluvio-glaciaires et glaciaires libère également du matériel cristallín. Voîcî un exemple d'analyse de sédiment de la Dranse :

$$
\begin{aligned}
& \text { galets (> } 2 \mathrm{~cm}) 56 \% \\
& \text { graviers }(2 \mathrm{~mm}-2 \mathrm{~cm}) 25 \%
\end{aligned}
$$$$
\text { sables et sablons } 19 \%
$$

Sur la fraction inférieure à $2 \mathrm{~mm}, 63 \%$ des grains sont supérieurs à $40 \mu$ avec une médiane de $0,63 \mathrm{~mm}$. Si on prend donc le sédiment total il y a au moins 4 ou 5 modes correspondant à des origines et des transports différents de matériaux. A cet égard les apports du Rhône sont beaucoup plus homogènes. Dans 1a fraction fine $(<40 \mu)$ 1a teneur en $\mathrm{CaCO}_{3}$ varie entre 35 et $40 \%$. Cette valeur quí est le double de celle du Rhône rend bien compte de la différence des bassins versants, nous verrons plus loin son influence sur la sédimentation du $1 \mathrm{ac}$.

\section{3. - Nature chimique des apports. \\ 4.3.1. - Filtration des eaux.}

Les apports du Rhône étant essentiellement sous forme de suspensions nous avons été amenés à faire les analyses chimiques sur celles-ci. Pour avoir les quantités nécessaires il nous a fallu concevoir une installation de filtration pouvant traiter de grandes quantités d'eau.

Le principe est de créer un vide sous le filtre, l'eau passe alors à travers celui-ci par dépression.

L'installation (figuresIV 8 et $\mathrm{IV}_{9}$ ) se compose de trois cuves d'une contenance de 1001 chacune. Dans les deux premières sont placées les eaux chargées, la troisième constitue le collecteur des eaux filtrées. Les eaux peuvent passer sur deux filtres montés en parallèles, on peut donc changer un filtre sans arrêter la filtration. Le vide est fait dans le collecteur au moyen d'une pompe Beaudoin protégée par une série de pièges à eau. En général nous avons filtré les eaux deux fois : une première fois sur filtre en papier "sans cendre", puis sur filtre Millipore HAWP de $0,45 \mu$ qui retient plus de $95 \%$ des suspensions. Pour remplir les cuves avec les eaux brutes ou filtrées une première fois, nous avons imaginé de chasser 


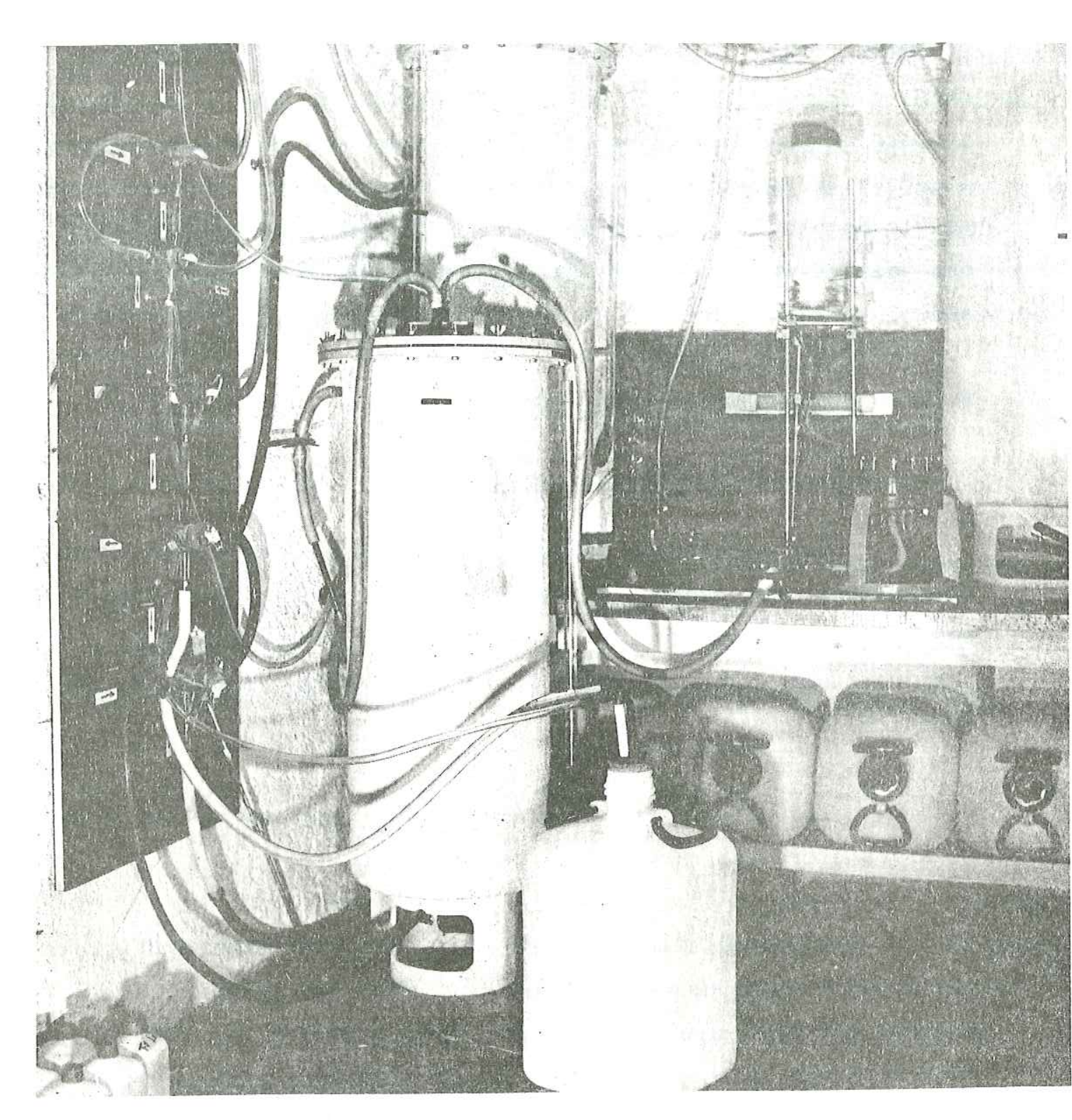

Installation de filtration

figure $I V_{8}$

1'eau par une surpression d'un gaz inerte : 1'azote. Il y a donc en parallèle au circuît général des eaux à filltrer un cîrcuit d'azote. On place sur le bidon de 301 un bouchon de caoutchouc avec une entrée d'azote et une sortie pour 1'eau, 1a surpression dans le bidon chasse l'eau dans le circuit et un ensemble de robinets permet de 1a répartir dans la cuve intéressée. De même la vidange des eaux filtrées du collecteur est également effectuée sans manutention par une surpression d'azote.

I1 était nécessaire de maintenir 1'installation à 1'abri des poussières : une fois que le filtre est mis sur son support 1 'ensemble est hermétiquement clos. De plus nous avons aménagé à 1 'intérieur de chaque cuve une douche permettant à de l'eau distillée de ruisseler le long des parois et de rincer la cuve après chaque filtration. Aux deux premiers circuits -eaux brutes et azotes'ajoute donc un troisième, celui de l'eau distillée. L'eau distillée est également chassée dans l'installation par de l'azote. Nous présentons sur la figure $\mathrm{IV}_{10}$ une coupe d'une cuve montrant les diverses entrées et sorties. Une jauge a été aménagée sur le côté. L'ensemble de la cuve est en fibre de verre ; le couvercle en PVC, rendu parfaitement étanche par un point torrique, est vissé sur 1a cuve. Enfin il est apparu utile de filtrer des petites quantités de 51 , nous avons donc aménagé sur le côté un "court-circuit", le bidon est renversé sur un entonnoir placé directement avant les fîltres.

La photo de 1 a figure $\mathrm{IV}_{8}$ permet de se rendre compte de 1'allure générale de 1'installation. Du moìs de Février 1968 à Juin 1970 environ 70001 de toutes provenances (Rhône, Dranse, mais aussi Congo, Mekong, Rhìn, Meuse, etc...) ont été filtrés. Si 1'installation ne nous a donné que des satisfactions plusieurs remarques peuvent cependant être formulées. Tout d'abord la forme intérieure des cuves devrait être en entonnoir afin qu'il y ait un minimum de dépôt au fond de celles-ci. Le système de douche intérieure serait 

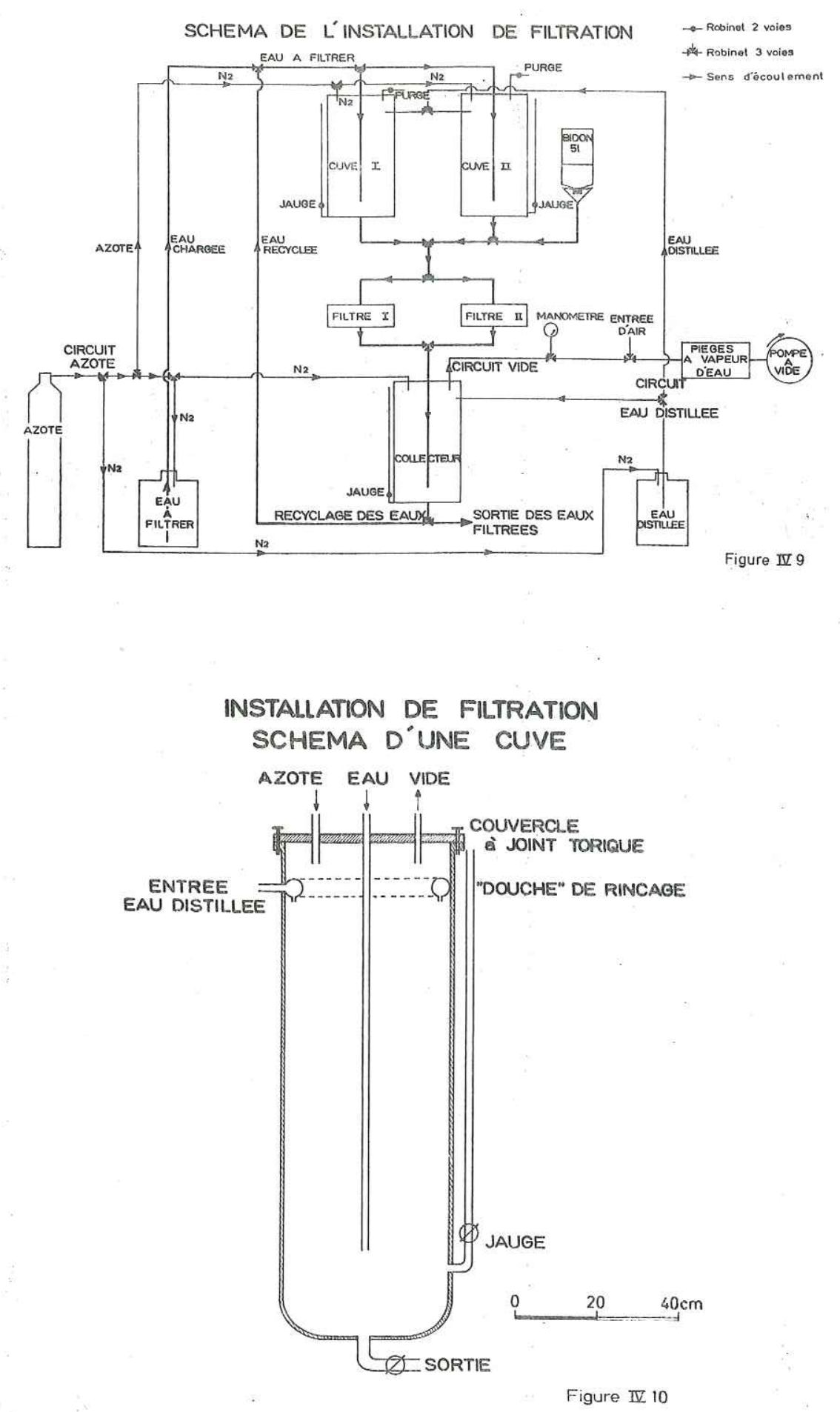

à perfectionner : la pression doit être plus forte. Enfin on peut poser le problème de la filltration par surpression ou dépression, 1a surpression nécessite des cuves et des tuyaux beaucoup plus résistants (nous avons d'ailleurs fait exploser une de nos cuves à cause d'une surpression), mais il est possible que le rendement soit meilleur.

\subsection{2. - Méthodes d'analyses.}

Comme les analyses chimiques des eaux, les analyses de suspensions et de sédiments ont été réalisées au laboratoire de chimie du C.R.G. par P. Blanc, ingénieur et A. Noir, technicienne. Les filtres comprenant les suspensions sont pesés à $105^{\circ} \mathrm{C}$ puis calcinés à $600^{\circ} \mathrm{C}$, et enfin analysés. La part des éléments apportés par les filtres eux-mêmes est très faîble. Les sêdiments sont aussi calcinés à $600^{\circ} \mathrm{C}$, 1a différence entre le sédiment fraîs et le sédiment calciné à $600^{\circ} \mathrm{C}$ correspond à la matière organique, les carbonates n'étant pas encore dissociés. Les éléments suivants ont été dosés :

$\mathrm{SiO}_{2}, \mathrm{Al}_{2} \mathrm{O}_{3}, \mathrm{CaO}, \mathrm{MgO}, \mathrm{Fe}_{2} \mathrm{O}_{3}$ ( $\mathrm{Fer}$ total), $\mathrm{MnO}, \mathrm{Na}_{2} \mathrm{O}, \mathrm{K}_{2} \mathrm{O}, \mathrm{TiO}_{2}$ et $\mathrm{P}_{2} \mathrm{O}_{5}$.

Après broyage et homogênéisation $100 \mathrm{mg}$ sont prêlevés et fondus avec $250 \mathrm{mg}$ de carbonate de lithium et $250 \mathrm{mg}$ d'acide borique. Le produit de fusion est repris par $5 \mathrm{ml} \mathrm{HCl} 1 / 2$ et $20 \mathrm{ml}$ d'eau, après dissolution totale le volume est ajusté à $100 \mathrm{ml}$. Sur cette solution sont dosés par spectrophotométrie d'absorption atomique

$\mathrm{SiO}_{2}-\mathrm{Al}_{2} \mathrm{O}_{3}-\mathrm{Fe}_{2} \mathrm{O}_{3}-\mathrm{MnO}-\mathrm{CaO}-\mathrm{MgO}-\mathrm{Na}_{2} \mathrm{O}-\mathrm{K}_{2} \mathrm{O}$.

Le titane et le phosphore sont dosés par colorimétrie : le titane par la méthode de 1'eau oxygénée, et le phosphore au molybdate avec réduction du complexe jaune par l'acide ascorbique. 
Enfin 1a perte au feu à $1000^{\circ}$ permet, par différence avec 1 a perte à $600^{\circ} \mathrm{C}$, de déterminer 1 a teneur du sédiment en carbonates. Dranse.

\subsection{3. - Analyse chimique des apports du Rhône et de la}

I1 convient tout d'abord de faire les remarques suivantes :

1) Le milieu des suspensions n'est pas un milieu bien mélangé, au contraire de l'eau les analyses chimiques pourront donc varier entre deux prélèvements consécutifs.

2) La composition chìmîque de l'apport d'une rivière est essentiellement fonction de la composition minéralogique. Or cette composition varie déjà pour un même prêlèvement entre les différentes fractions granulométriques.

3) Même pour une espèce minérale donnée on s'est aperçu récemment que la composition chìmique -surtout pour les éléments tracesétait fonction du diamètre des particules.

I1 ressort de ces considérations qu'une analyse de suspension ou de sédiment de rivières est beaucoup moins significative qu'une analyse d'eau. Nous considérons donc essentiellement les moyennes des analyses par éléments ainsi que les valeurs extrềmes portées sur le tableau $\mathrm{IV}_{4}$ 。

Les suspensions du Rhône et de la Dranse sont essentiellement des aluminosilicates, ainsi qu'en témoignent les fortes valeurs de $\mathrm{SiO}_{2}, \mathrm{Al}_{2} \mathrm{O}_{3}$ et $\mathrm{Fe}_{2} \mathrm{O}_{3}$ et le rapport $\mathrm{Al} / \mathrm{Fe}$. Les deux rivières ont donc des caractères principaux identiques néanmoins 1a Dranse est nettement plus calcaire puisque le total de CaO plus perte au feu entre 600 et $1000^{\circ} \mathrm{C}$ est de $35 \%$ (ce qui confirme la valeur obtenue par des analyses classiques pour la fraction fine du sédiment déposé) contre 15 \% au Rhône. Remarquons que ces valeurs correspondent à des échantillons sans matière organique. Pour les comparer de façon absolue aux analyses de sédiments il faut les majorer de quelques pour cents. Les autres éléments sont en proportion équivalentes pour les deux rivières puisque les rapports entre les êlểments sont les mêmes :

$\mathrm{SiO}_{2} / \mathrm{Al}_{2} \mathrm{O}_{3}=3,5$ pour le Rhône et 3,5 pour la Dranse

$\mathrm{Al}_{2} \mathrm{O}_{3} / \mathrm{Fe}_{2} \mathrm{O}_{3}=2,45$ pour le Rhône et 2,95 pour 1a Dranse

La différence la plus importante entre les deux rivières s'observe pour le sodium.

I1 est également intéressant d'étudier la dispersion des valeurs pour chaque élément. Pour le Rhône les plus constants sont dans 1 'ordre: $\mathrm{SiO}_{2}, \mathrm{TiO}_{2}, \mathrm{Al}_{2} \mathrm{O}_{3}, \mathrm{~K}_{2} \mathrm{O}, \mathrm{Fe}_{2} \mathrm{O}_{3}, \mathrm{MnO}, \mathrm{Na} \mathrm{O}_{2} \mathrm{MgO}, \mathrm{P}_{2} \mathrm{O}_{5}$ et c'est $\mathrm{CaO}$ qui varie le plus (de 1 à 5). Pour la Dranse les éléments constants sont dans l'ordre : $\mathrm{TiO}_{2}, \mathrm{SiO}_{2}, \mathrm{Al}_{2} \mathrm{O}_{3}, \mathrm{~K}_{2} \mathrm{O}, \mathrm{MnO}, \mathrm{Fe}_{2} \mathrm{O}_{3}$, $\mathrm{MgO}, \mathrm{CaO}, \mathrm{Na}_{2} \mathrm{O}$ et $\mathrm{P}_{2} \mathrm{O}_{5}$. Les quatre éléments $\mathrm{Ti}$, Si, $\mathrm{Al}$ et $\mathrm{K}$ sont donc remarquablement constants dans les deux rivières et leurs concentrations varient moins que du simple au double d'un prêlèvement à un autre.

Nous ne traiterons pas 1 'analyse des éléments traces ici, à titre indicatif nous donnons les valeurs moyennes de quelques analyses effectuées sur les suspensions au laboratoire de la S.N.P.A. à Pau par Kulbicky et à 1'E.S.C.M. de Mulhouse par Siffert (analyses en $\mathrm{ppm})$.

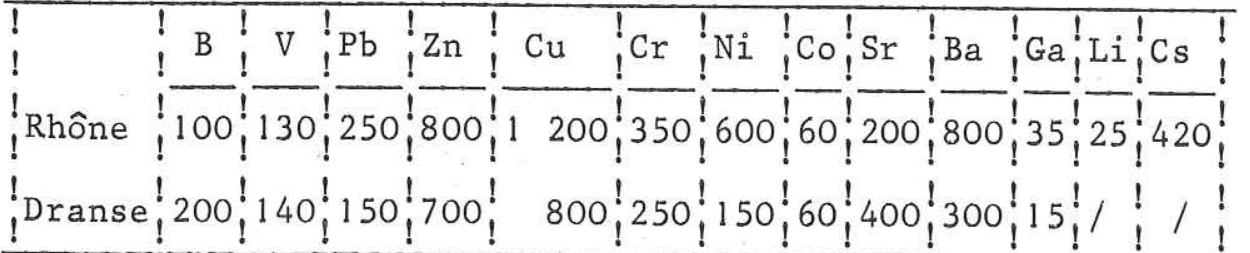


Ces valeurs ne sont qu'indicatives et représentent uniquement un ordre de grandeur (il y a souvent des valeurs 10 fois plus fortes que les autres, les causes en sont multiples, citons notamment la pollution des rivières). L'interprétation est donc encore plus délicate que celle des éléments principaux.

\section{A. 1.- - Bitan des apports chimiques en suspensions au Léman.}

Nous avons d'abord cherché à mettre en évidence des variations saisonnières ou en fonction du débît comme nous 1'avions fait pour les analyses d'eaux. Malheureusement vu la plus grande variabilité des suspensions un très grand nombre d'analyses serait nécessaire pour déterminer des lois de variations. Il semble ainsi d'après les analyses sur les crues de 1a Dranse que la turbidité aurait une influence assez grande et que cette relation composition chimiqueturbidités serait en plus spécifique de chaque épisode hydrologique (nous avions noté ce phénomène pour les éléments dissous mais il restait secondaire par rapport à la variation principale, due au débit).

Il en résulte que pour 1'établissement du bilan des apports en suspensions au Léman, nous avons pris les valeurs moyennes des analyses. Pour les affluents mineurs non étudiés nous avons pris des compositions chimiques analogues à celles de la Dranse.
TABLEAU IV $V_{5}:$ APPORTS MOYENS ANNUELS EN SUSPENSION

\section{DES ELEMENTS MAJEURS AU LEMAN}

(en milliers de tonnes)

sur la base de $6 \cdot 10^{6}$ t/an de suspensions minérales pour le Rhône $0,510^{6} \mathrm{t} / \mathrm{an}$ de suspensions minérales pour 1a Dranse $0,510^{6} \mathrm{t} / \mathrm{an}$ de suspensions minérales pour $1^{1}$ ensemble des autres rivières.

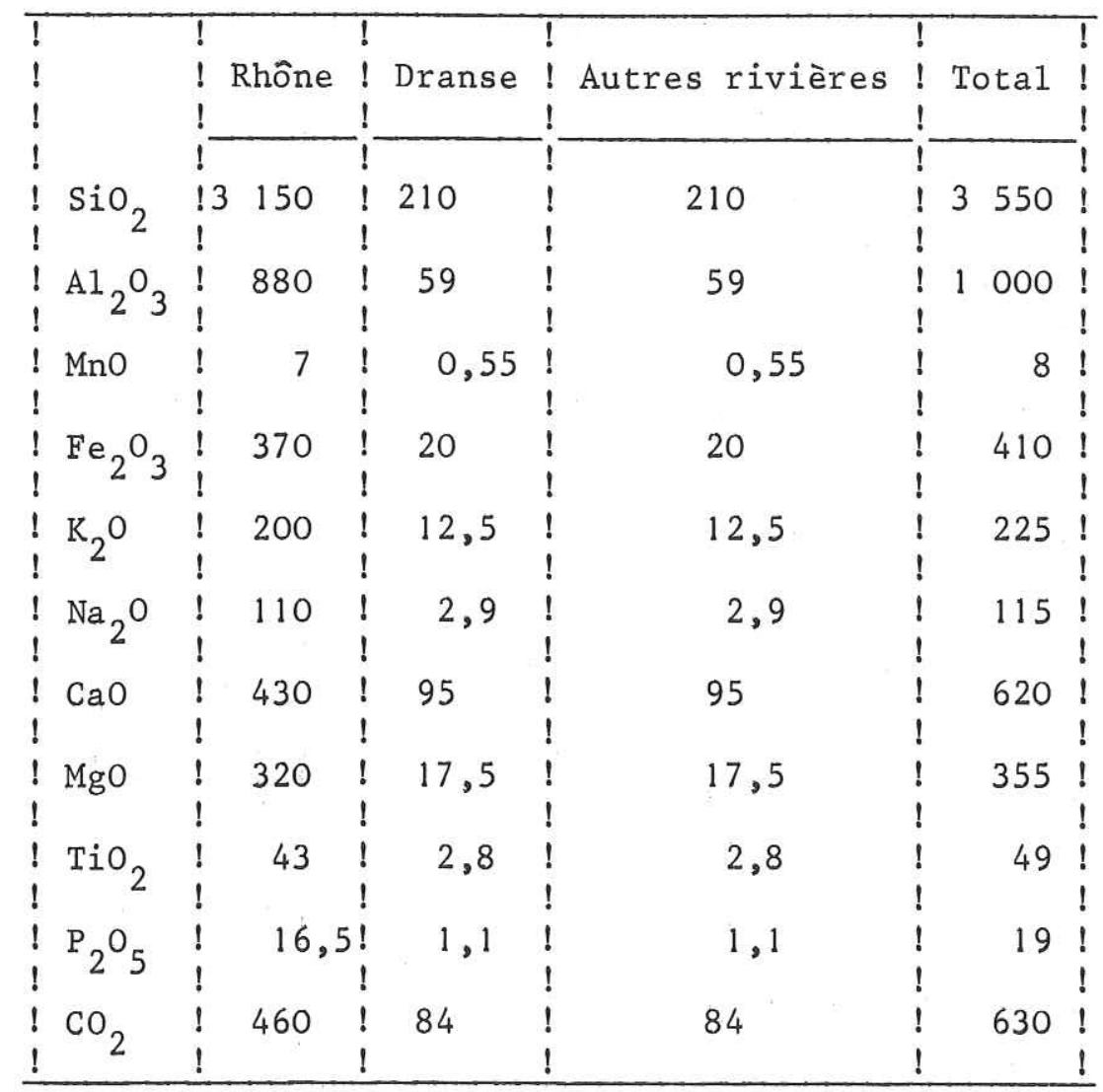




\section{5. - Etudes antérieures sur les sédiments du Léman.}

La première étude sérieuse a bien sûr étê réalisée par Forel dans sa monographìe sur le Léman (1895). Depuìs un grand nombre de chercheurs ont étudié les sédìments du Léman. Entre les deux guerres citons les nombreux travaux de 1 'Université de Genève : Collet (1925), Parejas (1929), Romieux (1930), Joukovski et Buffle (1938). Ces travaux ont essentîellement porté sur le Petit Lac, mais les études de Romieux sur les carbonates, et de Parejas sur les varves intéressent tout $1 \mathrm{e}$ lac. Depuis une vingtaine d'années peu de travaux ont été réalisés en dehors de ceux menés au C.R.G. par C. Serruya (1969), J. Sauvage (1969), C. Vergnaud-Grazzini (SerruyaVergnaud -Grazzini, 1967) quî ont portê sur la diagénèse, 1'analyse palynologique et micropaléontologique. Enfin les chercheurs de la compagnie Shell ont effectué une campagne de carottage complète dans le Haut-Lac doublée de prospection sismique (Houbolt et Jonker, 1968). Le Léman est donc déjà bien connu et on peut estimer le nombre de carottes réalisées à plusieurs centaines. Néanmoins beaucoup de points restent encore à préciser : la composition chimique générale des vases n'a été étudiée que très partiellement (Zender 1908, Monod 1956), de même que 1'étude géochimique du type de sédimentation, ce sont ces points que nous avons essayé de développer. Pour cela après avoir caractérisé les apports des rivières nous nous sommes intéressés principalement aux sédiments superficiels.

\section{6. - Modes de prélèvements.}

Nous avons utilisé trois types d'appareils : la benne Eckman-Birge de $20 \mathrm{~cm}$ X $20 \mathrm{~cm}$ d'ouverture qui perturbe peu le sédiment, le carottier Jenkins-Mortimer dont la pénétration dans le sédiment est presque parfaite, et enfin le carottier à mouton Züllig $(\varnothing 36 \mathrm{~mm})$. Ce dernier de par son mode d'enfoncement peut produire

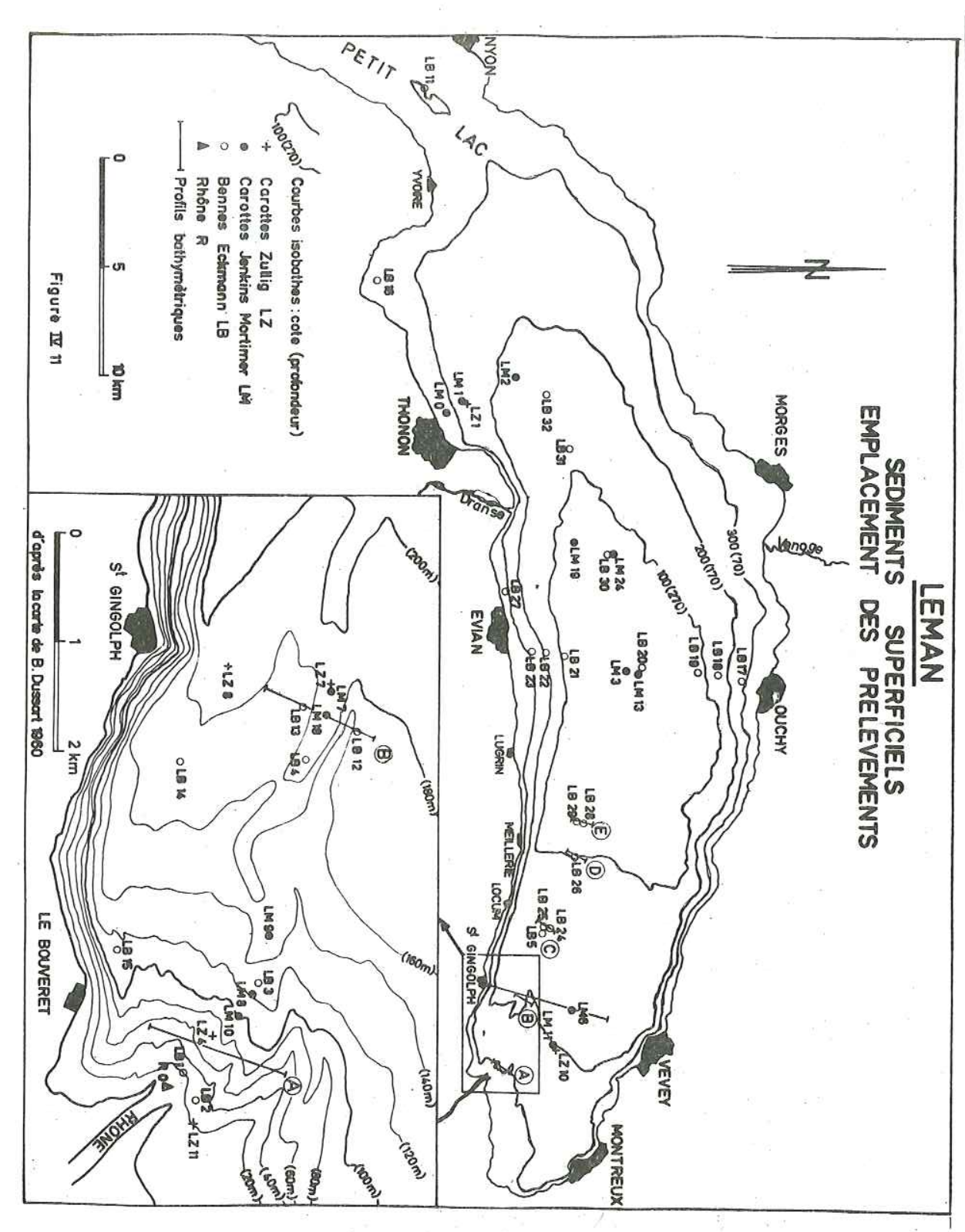


des tassements dans le sédiment. Comme les prélèvements d'eau, les prises de sédiments ont été réalìsées par G. Olìvier, technicien, à bord du bateau du C.R.G.,1e Moïse, spécìalement équipé pour la recherche sur les lacs. Les prélèvements dont nous parlerons ici sont localisés sur la fìgure $I_{1 j}$. Nous nous sommes repérés par alignements et surtout à l'écho-sondeur Sadir-Carpentîer, en particulier pour la région du canyon du Rhône, où nous avons pourtant bénéficié de la carte établie par Dussard (1961) nettement plus précise que celle de Delebecque-Hornlìmann établìe à la sonde. Dans la plupart des cas nous avons pu caractériser dans les bennes et le carottier Mortimer une partie supérỉeure et une partîe inférieure.

\section{7. - Etude sédimentologique du Haut-Lac.}

\subsection{1. - Morphologie du Haut-Lac.}

C'est la portion du lac la plus intéressante au point de vue de 1a sédimentation. En effet plusieurs types de sédîmentation coexistent et se superposent : sédìmentation détrîtíque fine provenant des apports du Rhône, sédimentation bìochìmique, sédimentatîon détritĩque grossière canalìsée par le canyon du Rhône.

Le Haut-Lac est caractérísé par le delta sous lacustre du Rhône échancré par de nombreux ravins sous-lacustres ou canyons plus ou moins comblés. Le principal canyon actuel a étể découvert en 1885 par Hörnlimann qui venait d'en mettre en évìdence un semblable dans le lac de Constance. Le rôle du canyon a été prêssenti par Forel mais c'est Kuenen (1950, p. 510) qui le premier vit la possibilité de courants de turbidité passant dans le canyon.

Le canyon débute près de 1 'embouchure du Rhône (figure $I_{11}$ ) avec une assez forte pente ( $8 \%$ sur $2 \mathrm{~km}$ ) qui diminue progressivement $(1 \%$ sur $10 \mathrm{~km})$, il aboutit dans une vaste cuvette plate à $309 \mathrm{~m}$ de profondeur ("plaine centrale" de Fore1). La dénivellation entre les bords et le fond du canyon est de $30 \mathrm{~m}$ au maximum, la largeur est de 

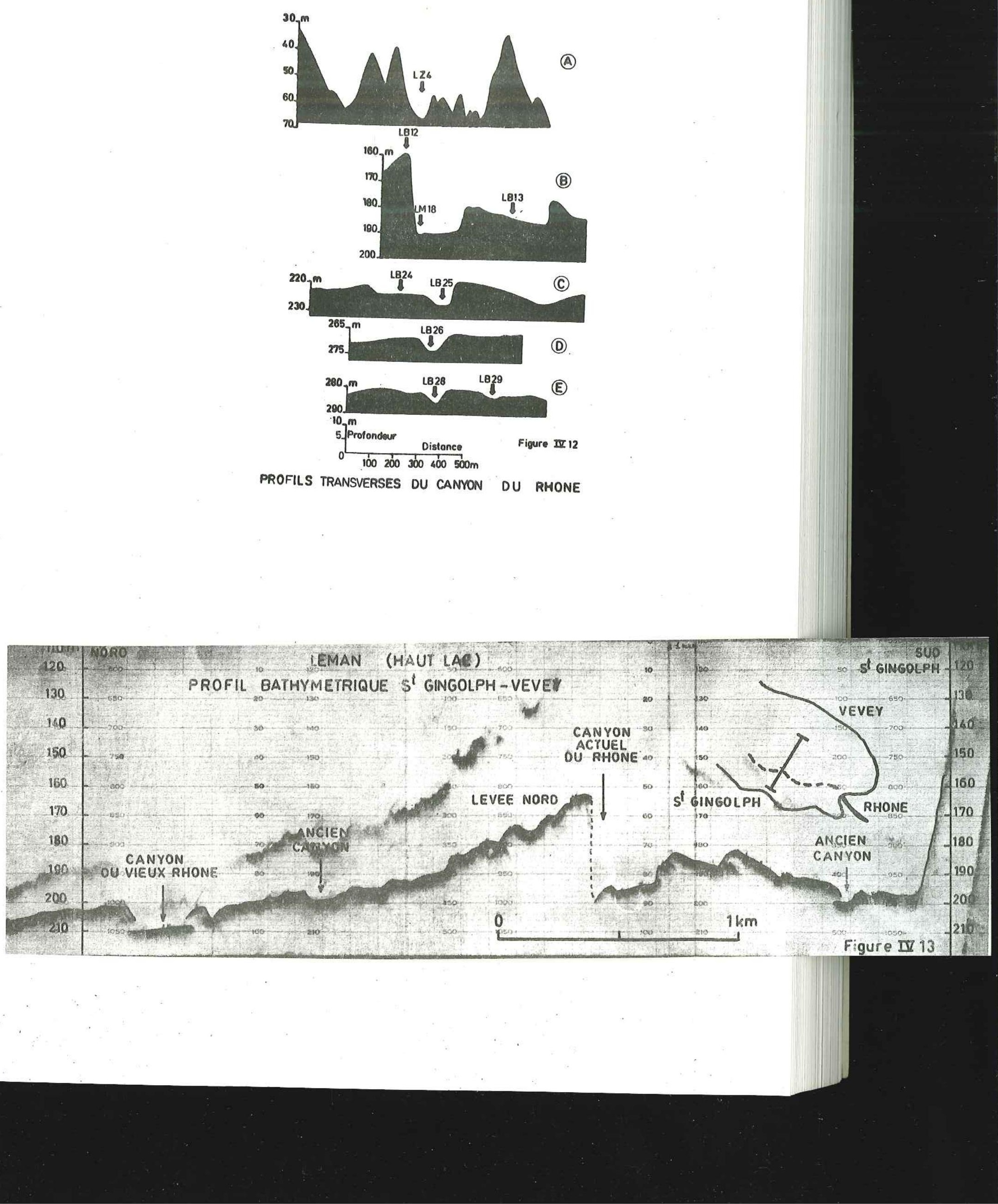
$250 \mathrm{~m}$, les levées du canyon sont souvent assymétríques. Cette morphologie générale est très analogue à celle dếcrîte pour les formes de relief océaníque (Gougenheìm 1970) et, quoìqu'à l'échelle océanique le canyon du Rhône devrait plutôt s'appeler "ravine", il semble bien que nous pouvons luî laisser son nom vu le rôle qu'il a à l'échelle du Léman. Il a toutes les caractêrîstìques des

canyons sous-marîns classìques : en plus des "levées" très nettes, on peut aussi luỉ défînîr un "cône" traversé par des "chenaux"。 Le delta du Rhône serait alors semblable à une "pente continentale" et la plaine centrale à un "bassîn". Les profils bathymétriques de la figure $I_{12}$ (localissés sur le plan de prélèvement, figure $I_{11}$ ) montrent bien la morphologìe du canyon : fond plat, levée dissymétrique, et nombreux chenaux dans le cône vers $270 \mathrm{~m}$ de profondeur. Notons également 1a grande îrrégularîtế des fonds sur 1a coupe A près de l'embouchure, en effet le canyon ne s'indîvîdualìse vraíment qu'à $1 \mathrm{~km}$ de celle-cí.

De nombreux autres canyons partìellement comblés s'observent au nord (canyon du Vỉeux Rhône) comme au sud du canyon actuel. Ils ne sont plus en fonctionnement et la sédimentation fine les comble peu à peu. Sur le profil St-Gingolph-Vevey (figure $\operatorname{IV}_{13}$ ) on dìstingue très bìen au moìns troìs anciens canyons, Houbolt et Jonker (1968) en dénombrent six. Sur ce même profil aìnsì que sur 1e profil $\mathrm{B}$ (figure $\mathrm{IV}_{12}$ ) on remarque que le bord méridional du canyon actuel au niveau de St-Gingolph a une morphologie tout-àfait différente du bord septentrional. Il y a un replat de $400 \mathrm{~m}$ puis une levée de $10 \mathrm{~m}$ de haut beaucoup moins importante que la levée nord. D'autres coupes -non présentées ici-montrent même un petit ravin. Peut-être avons nous là la preuve d'un surcreusement du canyon actuel dans un lit plus ancìen dont cette levée serait un témoin. Une campagne de sismique continue au sondeur de vase est prévue pour étudier plus en détaîl ces intêressants phênomènes.

\subsection{2. - Fonctionnement du canyon du Rhône.}

\section{Les types de dépôts rencontrés.}

Outre les prêlèvements à 1 a benne et au carottier Mortimer, dont les analyses physíques sont reportées au tableau $\mathrm{IV}_{3}$ en annexe de ce chapitre, nous avons effectué des prélèvements au carottier Züllig dont le log descriptìf est reporté aux figures $I_{14}$, IV ${ }_{15}$ et $\mathrm{IV}_{16}$ (m est $1 \mathrm{a}$ médiane). Les courbes granulométriques typiques sont reportées sur la figure $\mathrm{IV}_{7}$.

La carotte $\mathrm{LZ}_{7}$ est caractéristique du fond du canyon : 1e niveau inférieur est composé d'alternances non régulières de sablons plus ou moins fins et de vase, il luì succède brusquement un niveau de sable fin granoclassê comportant quelques débrîs végêtaux. De tels sédiments s'observent tout au long du canyon jusqu'au cône où des sablons de médianes supérieures à $100 \mu$ existent encore. Houbolt et Jonker ont même trouvé des médianes de $230 \mu$. Dussart (1966) fait état d'une carotte prélevée à $300 \mathrm{~m}$ de profondeur avec encore "275 mm de sable rhodanien micacé granoclassé", puis au-dessous une zone assez bien stratifiée avec des varves de 1 à 4 millimètres. Notons que Melle Coìt (in Romieux, 1930) avait déjà trouvé en 1925 un lit de $1,5 \mathrm{~cm}$ de sable grossier dans une carotte située dans 1 'axe du canyon du Rhône par plus de $300 \mathrm{~m}$. Il n'est pas possible de suivre horizontalement un niveau dans les dépôts du fond du canyon, les phênomènes d'érosion rendent ce dernier très hétérogène. Nous n'avons pas remarqué comme Dussart (1961) que "les sédiments étaient d'autant plus gros dans le chenal du Rhône qu'on s'éloìgne de l'embouchure". Au contraire Houbolt et Jonker présentent des courbes granulométríques pour le fond du canyon (channe1) plus grossières que pour le cône central (central fan). Quelquefois même les dépôts sont mal classés et plurimodaux, ainsi qu'on peut le voir pour la partie inférieure de la benne $\mathrm{LB}_{\mathrm{F}}$ (figure $\mathrm{IV}_{7}$ ).

Le même genre de dépôts grossiers s'observe également dans 
LEMAN

DESCRIPTION DE CAROTTES CARACTERISTIQUES

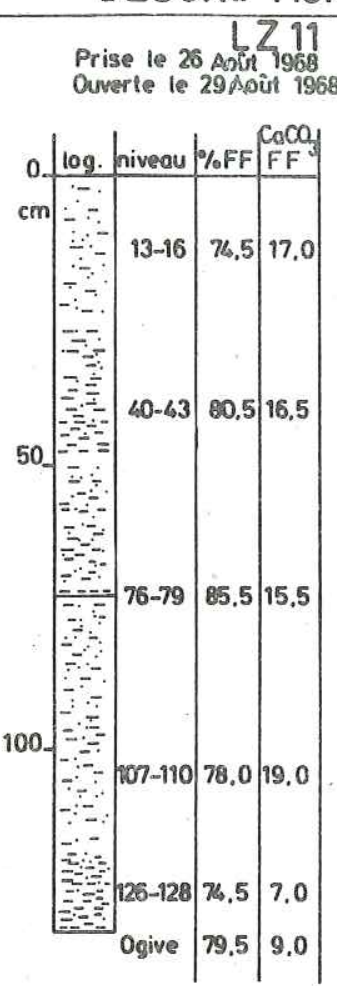

Figure III 14

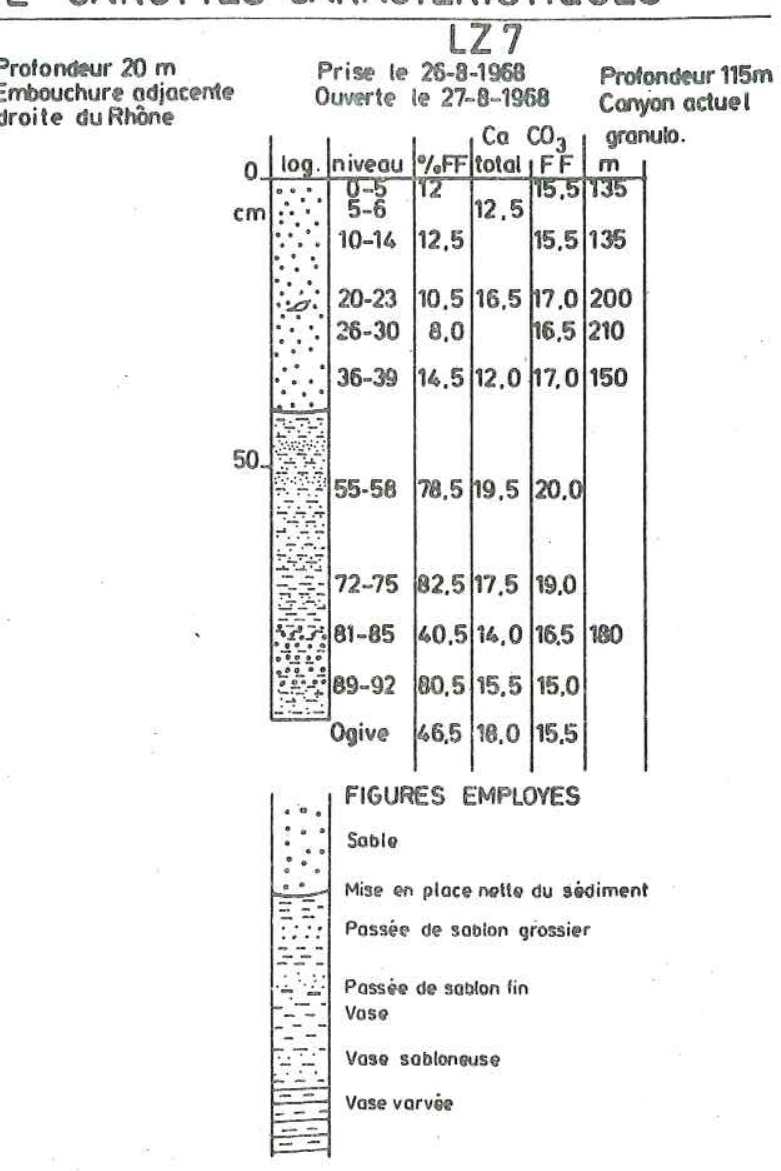

les anciens canyons. La carotte $\mathrm{LZ}_{8}$ prìse dans 1 'ancìen canyon sud devant St-Gingolph montre, sous 80 centimètres de vase, des séquences de sablons plus ou moins granoclassés et des niveaux vaseux varvés. Des traces d'érosion sont nettement vissibles, enfín nous avons trouvé à $132 \mathrm{~cm}$ de la surface un morceau de scorie charbonneuse sur lequel nous reviendrons. Houb̄olt et Jonker font également état de carottes identîques dans les anciens canyons vers Vevey.

Remarquons enfin que les bennes réalìsées dans le canyon en hiver $\left(\mathrm{LB}_{2}, \mathrm{LB}_{4}\right)$ montrent d'abord une couche de quelques centimètres de sablon vaseux, rîche en eau et en débrìs végétaux (feuilles, etc...) qui recouvre les niveaux nettement sableux.

\section{Les turbidites.}

Les sédiments grossiers ont donc été rencontrés dans le canyon actue1, son cône, et sous des dépôts de vases dans les anciens canyons. Partout ailleurs dans le Haut-Lac les dépôts récents sont constitués par des vases plus ou moins varvées (carotte $\mathrm{LZ}_{10}$, figure $\left.\mathrm{IV}_{16}\right)$. A 1 'ancienne embouchure du Rhône la sédimentation fine a déjà recouvert les apports grossiers (carotte $\mathrm{LZ} Z_{11}$ ). Nous avons vu au chapitre précédent que le canyon est le siège de courants turbides très chargés en suspensions, très localisés et provenant directement du Rhône, ce sont ces courants qui ont transporté puis déposé les sédiments grossiers décrits précédemment. Ces dépôts ont la plupart des caractéristiques des turbidites telles qu'elles ont été décrites par Kuenen (1964).

- Taille des particules : Le diamètre moyen des particules va de 50 microns à 420 microns. Des grains de quartz de $1 \mathrm{~mm}$ ont été rencontrés.

- Granoclassement : Nous ne 1'avons pas observé dans le canyon 1ui-même mais Houbolt et Jonker en font état pour les sédiments du cône. 
LEMAN

DESCRIPTION DE CAROTTES CARACTERISTIQUES

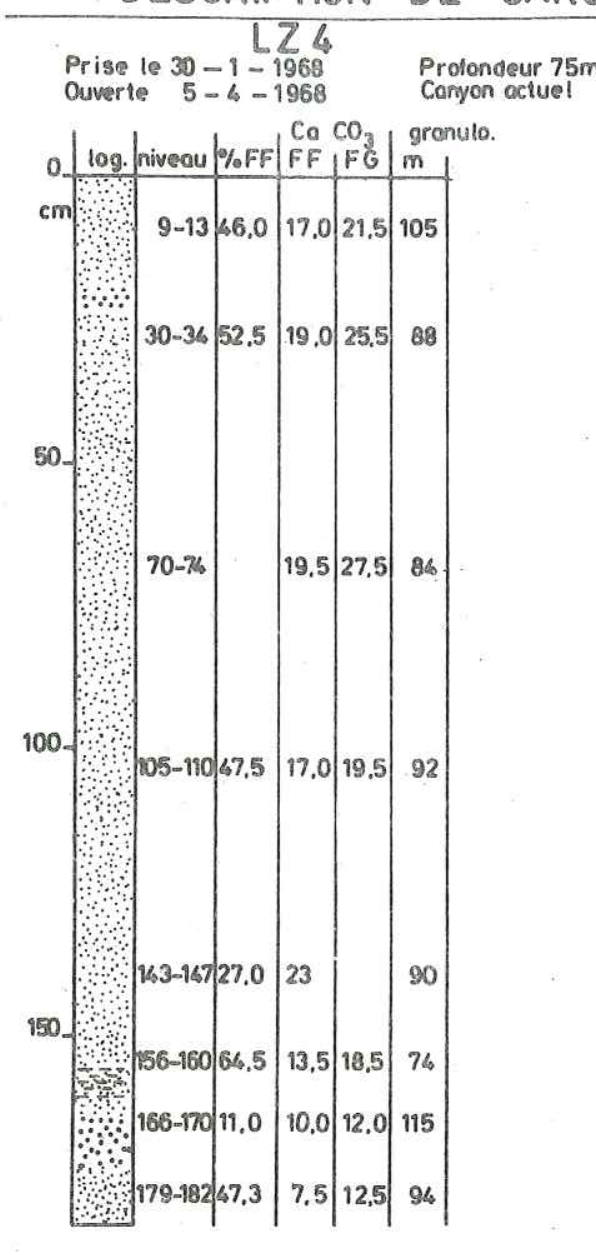

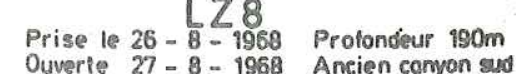

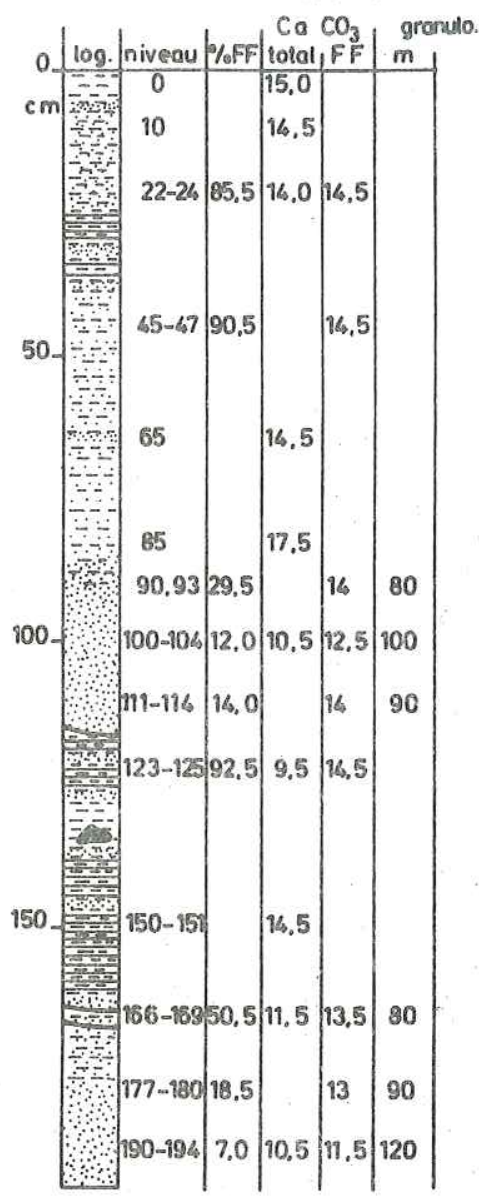

Figure III 15
- Triage : Le triage est en général bon, mais quelquefois médiocre $\left(\mathrm{LB}_{25}\right.$ et $\left.\mathrm{LB}_{28}\right)$.

- Débris végétaux : I1s sont très nombreux dans le sablon fin : fragments de feuilles, d'herbes, même de roseaux et un os $\left(\mathrm{LB}_{4}\right)$.

- Figure de sédimentation : En raison de notre mode de prélèvement nous n'avons pas pu les étudier. L'épaìsseur des lits de sablon varie de 1 à $2 \mathrm{~cm}$ à plus de $40 \mathrm{~cm}$. La plupart du temps les lits de sablon et a fortiori ceux des sables débutent bien nettement. Certaîns se terminent brutalement par une sédimentation vaseuse, il semble qu'il y ait alors érosion de la partie supérieure du lit avant le dépôt de la vase.

- Glissements : Nous avons trouvé dans la carotte $\mathrm{LZ}_{7}$ sur $9 \mathrm{~cm}$ d'épaisseur un mélange de $60 \%$ de sable moyennement classé et de $40 \%$ de vase fine il semble bien que ce mêlange soît dûa un g1issement.

- Fréquence des courants : Comme nous l'avons dit au chapitre précédent, ces courants sont essentiellement liês aux crues du Rhône qui ont lieu chaque année en Juin et Juillet. Mais les crues d'hiver, plus rares, doivent provoquer les mêmes phénomènes. Entre les courants il y a une sédimentation détritique fine, riche en eau et en matières organiques, c'est ce que nous avons rencontré à la surface des bennes $\mathrm{LB}_{2}$ et $\mathrm{LB}_{4}$, effectuées en Janvier 1968 après une période de plusieurs mois sans crues. Le problème de la fréquence des courants se pose aussi pour les "anciens canyons", il est vraisemblable qu'avant la stabilisation du lit du Rhône les grandes crues $\left(2000 \mathrm{~m}^{3} / \mathrm{s}\right.$ ) devaient y provoquer des courants de turbidité. La morphologie assez "frâ̂che" du canyon du Vieux Rhône (figure $I V_{13}$ ) en est le témoin. Pour l'ancien canyon, le plus méridional, près de St-Gingolph, les dépôts grossiers (carotte $\mathrm{LZ}_{8}$ ) sont recouverts par 
LEMAN DESCRIPTION DE CAROTTES CARACTERISTIQUES

\begin{tabular}{|c|c|c|}
\hline & & \\
\hline $\begin{array}{l}\text { Prise le 22-1-9 } \\
\text { Ouverle le 1-4-4- }\end{array}$ & $\begin{array}{l}\text { LZ I } \\
1968\end{array}$ & $\begin{array}{l}\text { Protondeur } 100 \mathrm{~m} \\
\text { large de Thonoon }\end{array}$ \\
\hline 0 log hiveoul & 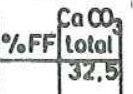 & \\
\hline 㗐 $12-43$ & 98.531 & \\
\hline 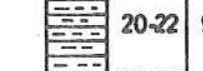 & 98.030 & \\
\hline 琵- $30-32$ & 97.031 .5 & \\
\hline 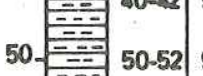 & $38,0,3$ & \\
\hline 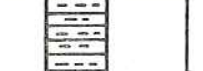 & & \\
\hline 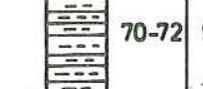 & \begin{tabular}{|l|l|l|}
97.5 & 31
\end{tabular} & \\
\hline 谓 $90-92$ & \begin{tabular}{|l|l|}
97,5 & 31
\end{tabular} & \\
\hline $00=0$ & & \\
\hline${ }^{-1}=10-112$ & 96,529 & \\
\hline 踏130-932 & \begin{tabular}{ll|l}
97.5 & 28
\end{tabular} & \\
\hline & & \\
\hline $150=150452$ & $\begin{array}{l}96.0 \\
26\end{array}$ & \\
\hline 望语 & & \\
\hline 172-873 & 97.529 & \\
\hline $\begin{array}{l}-180-182 \\
E=-186-189\end{array}$ & & \\
\hline $\begin{array}{l}\text { Epaisseur des vor vo } \\
\text { varves gofise } \\
\text { varves noire. }\end{array}$ & 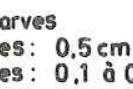 & \\
\hline
\end{tabular}

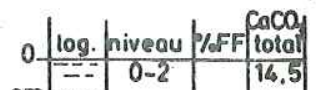

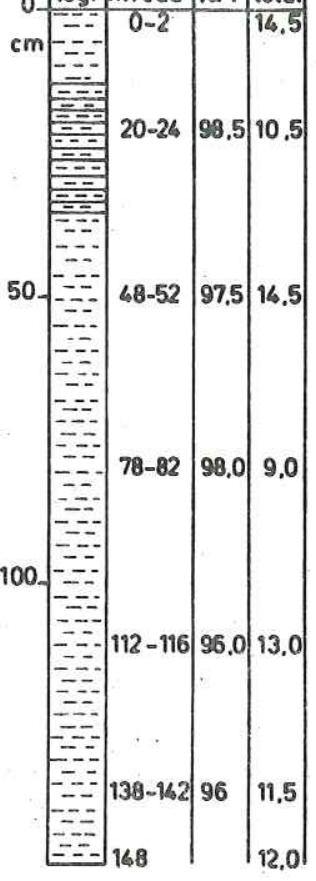

Epaisseur des vorves:
varves grises
verves noires $\mathrm{cm}: 3.5 \mathrm{~cm}$

Figure 正 16
$80 \mathrm{~cm}$ de vase fine avec quelques litts de sablons, il semble donc que ce canyon n'aît pas fonctionné depuîs une cînquantaîne d'années.

\section{Origine des canyons dans le Léman.}

E1le a été longtemps contreversée : Forel pense à un creu-

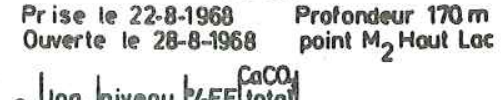

\section{Autres types de dépôts grossiers.}

En dehors des sédiments grossîers rencontrés dans le canyon actuel, son cône et au niveau des anciens canyons, les sables et les sablons ne s'observent guère qu'aux embouchures des autres rivières, Dranse, Venoge, etc... où les apports plus grossiers ont constitué des deltas. De tels sédiments ont été recueillis dans la benne $\mathrm{LB}_{17}$ devant Ouchy par $103 \mathrm{~m}$ de profondeur : la médiane de la fraction grossière est de $250 \mu$ (figure $I V_{7}$ ), il s'agît d'un sable noir bien classê, avec des gros débris végêtaux ; il correspond sans doute aux apports du Flon canalisés par une "ravine"。 Enfin les sédiments grossiers peuvent provenîr de glissements à partir des berges, comme le montre bien le prélèvement réalisé devant le Bouveret $\left(L B_{15}\right)$ : le 
sabie, quî ne représentait d'atileurs que $20 \%$ de la laraction totale étaît três grossier et plurîmodal (voîr la courbe cumulêe sux la figure IV7), i. y avait jusqu’à des cailioux et des scories. Enfin il nous faut mentionner. 1a présence tout-à-fait anormale de graviers wcertains avec des traces de goudrons- dans des vases très fines et ceci loin des rives. Nous en avons trouvê notamment dans les prêlèvements $\mathrm{LM}_{1}$ et $\mathrm{LB}_{23}$. Forel avaît đéjà remarqué ce phénomène, il ne paut s'agir que d'apports accidenteis provenant de nombreux bateaux qui sỉllonnent le Lểman.

\subsection{3. - La sédimentation fine dans le Iéman.}

En dehors de ces dépôts que Forel appelle "aliluvion gros" sière" la majeure partîe du fond du Lểman est recouverte de dêpôts três fins, l"alluvion impalpable". Cette forme de sédìmentation se rencontre partout dês qu'on s'êloigne un peu des cotes oủ $1^{\gamma}$ action des vagues et des courants de retour empêche le dểpôt. La stratigraphìe de ces dêpôts est maîntenant bìn connue après les travaux de J. Sauvage (1969) sur les carottes de C. Serruya (1969), qui on permis de reconnaître les diffêrentes phases palếosilvatiques jusqu'à l'oldest Dryas. Pour quelques prélêvements il y a des lacunes montrant d'anciennes périodes d'êrosion ou de non-dêpôt, surtout dans les zones côtières et le Petìt-Lac.

Cette sédimentation fine se rencontre partout dans 1 Lëman, nous avons porté sur la figure $\mathrm{IV}_{16}$ la description des carottes $L Z_{1}$ et $L Z_{1}$ a réalisées respectivement en face de Thonon et en İac de Vevey. Pour les deux carottes $98 \%$ du sédîment est infếrieur à 40 microns. Les deux dêpôts sont varvếs : il y a alternance de varves grises et noires. Pour $\mathrm{LZ}_{1}$ un ensemble de deux varves fait environ $0,8 \mathrm{~cm}$ et pour $\mathrm{LZ}_{10}$ de 3 à $4 \mathrm{~cm}$. Parejas (1929) a présentê une carte des êpaisseur's de ces varves pour le Haut-Lac, mais pour les mểmes endroits ses valeurs sont nettement plus faibles que les nôtres et celles d'Houbolt et Jonker. L'épaisseur des varves est maximale vers le Rhône où elle atteìnt plusieurs centimètres et diminue rapidement pour n'être que de quelques mîllimètres au niveau de Meîllerie. Pour Serruya il y a deux types de varves : les varves granulométriques dans la zone d'influence du Rhône, ce sont celles décrites par Parejas, et les varves biochimiques du Grand Lac et du Petit Lac. Le problème de la formation des varves n'est pas encore bien résolu,dans le cas du Léman il nécessiterait une étude microstratigraphique avec des analyses granulométriques et chimiques varve par varve.

Houbolt et Jonker (1968) ont pu effectuer 1'analyse granulométrique totale des sédiments fins. Les valeurs moyennes des mêdianes sont les suivantes:

$$
\begin{aligned}
& \text { partie supéríeure du delta du Rhône : } 6 \text { à } 20 \mu \\
& \text { partie moyenne } \quad \text { : } 4 \text { à } 8 \mu \\
& \text { partie inférieure } \quad \text { " } 3 \mu \\
& \text { plaine centrale } \quad \text { : } 2 \text { à } 4 \mu
\end{aligned}
$$

Par contre sur les flancs de la cuvette les sédiments beaucoup plus fins témoignent d'un affaiblissement net du courant général au niveau de la partie moyenne du delta les mêdianes sont déjà inférieures à $2 \mu$ 。

\section{8. - Etude chimique des sédiments actuels.}

\subsection{1. - Travaux antérieurs.}

Les premiers résultats sont cîtés par Forel et Delebecque. Ces analyses parcellaires sont très îrrégulières néanmoins Forel remarque déjà que "la richesse en sîlìcate va en diminuant à mesure qu'on s'avance dans le lac en s'éloîgnant du Rhône" (Le Léman, tome 1, p. 128) et que la "chaux et la magnésie évoluent en sens inverse que les silicates", nous verrons ce qu'il faut penser de cette association. Zender (1908) fit une analyse plus complète de la vase du Léman ( $\mathrm{Si}, \mathrm{Fe}+\mathrm{A} 1$, 
$\mathrm{Ca}, \mathrm{Mg}, \mathrm{CO}_{2}$ ) mais malheureusement cet auteur choisiit un sêdiment prélevé par $20 \mathrm{~m}$ de fond dans le Petît Lac, ses rêsultats ne sont donc pas du tout représentatìfs de $1^{\prime}$ ensemble du Léman. Plus tard Joukowski et Buffle (1938) fîrent une très ìntêressante étude sur des sondages effectués à Ruth toujours dans le Petit Lac, aussi leurs résultats appellent les mêmes remarques. Enfin Monod (1956) dans son étude sur la poliution apportée par le Flon prêsenta des analyses beaucoup plus complètes $\left(\mathrm{SìO}_{2}, \mathrm{Al}_{2} \mathrm{O}_{3}, \mathrm{Fe}_{2} \mathrm{O}_{3}, \mathrm{CaO}, \mathrm{MgO}\right.$, $\mathrm{P}_{2} \mathrm{O}_{5}, \mathrm{SO}_{3}$, perte au feu). Maìs cette étude est cîrconscrite à une petite zone autour de 1 'embouchure de la rìvì̀re. Il faut placer à part la très intếressante étude de Romîeux (1930) quî n'a porté que sur les carbonates maîs quîn întéresse tout le lac. La fìgure $\mathrm{IV}_{17}$ prếsente ses rêsultats sous une forme plus pratique Enfin dans son travaìl sur les sêdîments du Lẻman, Serruya (1969) a essayé de voîr les corrêlations entre les paléoclimats dëfinis par la palynolonie et les éléments traces des sêdìments, aucune analyse d'élêments majeurs n'est prêsentée. Aucune étude génêrale de la composition chìmîque des sédìments du Léman n'avait donc été réalisée.

L'étude que nous présentons icî porte sur 37 analyses, de 1 a fractìon infêrieure à $40 \mu$, de sëdìments prélevês sur tout le lac (voir la carte de la figure $\mathrm{IV}_{1 i}$ ). Les analyses des éléments majeuxs des sêdiments sont reportées sur le tabieau $\mathrm{IV}_{6}$, les moyennes et les valeurs extrêmes sont sur le tableau $\mathrm{IV}_{4}$, tous deux en annexe du chapitre. Le nombre d'analyses réalisées est encore bien insuffisant pour effectuer une étude complète de la chimie des vases du Léman : la variabiliitế des facies est très grande; en prenant les sédiments superficiels on n'est pas toujours sûr de prélever des sêdiments actuels (êrosion, absence de dépôt); enfin même pour un dépôt fin et régulier comme celuî de la plaine centrale, on peut noter des variations de 1 'ordre de $10 \%$ en quelques centimètres comme pour le prélèvement $\mathrm{LM}_{13}$ (voir tableau $\mathrm{IV}_{6}$ en annexe). Aussí nous n'avons donné ícî que les grandes lignes des variations chimiques, sans entrer dans les études de détaìi.

\subsection{2. - Caractères chimiques des sédiments du Léman.}

Les sédiments du Léman ont les mêmes caractères chimiques génếraux que ceux des apports : $\mathrm{SiO}_{2}$ est prédominant, puis $\mathrm{CaO}$, $\mathrm{Al}_{2} \mathrm{O}_{3}$ et $\mathrm{CO}_{2}$. En général les sêdìments du Léman sont donc essentiellement composés d'aluminosilicates, de quartz et de carbonate de calcium, mais la proportion de ces constituants varie considêrablement. Romieux avait déjà mîs en évîdence la variation des carbonates. Sur la figure $\mathrm{IV}_{17}$ on voìt nettement 1 'accroissement général des carbonates de calcîm au fur et à mesure qu'on s'éloìgne du Rhône. On note également une augmentation nette vers les zones cotières de faibles profondeurs, augmentation encore accentuée dans la zone du delta de la Dranse par les apports très calcaires de celle-ci ( $35 \%$ de $\mathrm{CaCO}_{3}$ dans la fraction fine). L'évolution de 1a silice est exactement l'inverse ainsì que le montre nettement la figure $\mathrm{IV}_{18}$ : 1a teneur en $\mathrm{SiO}_{2}$ décroît lorsqu'on s'éloigne du Rhône, de plus on note bien la différence observée entre les sédiments cotiers plus riches en $\mathrm{CaCO}_{3}$ et les sédiments profonds. Enfin remarquons la différence -normale- entre les dêpôts de turbidites $\mathrm{LB}_{29 \mathrm{~F}}$ et $\mathrm{LB}_{28 \mathrm{~F}}$, dont la composition est beaucoup plus proche de celle des apports du Rhône, et les sédiments fins voisins.

La corrélation entre les différents éléments a été étudiée en comparant pour 9 sédiments répartis sur tout le lac, les rapports des éléments successivement à la silice et au $\mathrm{CO}_{2}$ (ce dernier a été calculé par différence entre les pertes à $1000^{\circ} \mathrm{C}$ et à $600^{\circ} \mathrm{C}$ ). Nous avons reporté ces résultats au tableau $\mathrm{IV}_{7}$. On voît clairement que presque tous les éléments sont liês à la silice, leur rapport à cet élément étant peu variable. Les éléments les plus liés à la silice sont dans l'ordre: $\mathrm{Al}, \mathrm{Fe}, \mathrm{K}, \mathrm{Ti}, \mathrm{Na}$ et $\mathrm{Mg}$. Outre le calcium, le phosphore et le manganèse sont à part, nous y reviendrons. Les rapports des éléments au $\mathrm{CO}_{2}$ sont très variables : sauf pour le calcium 

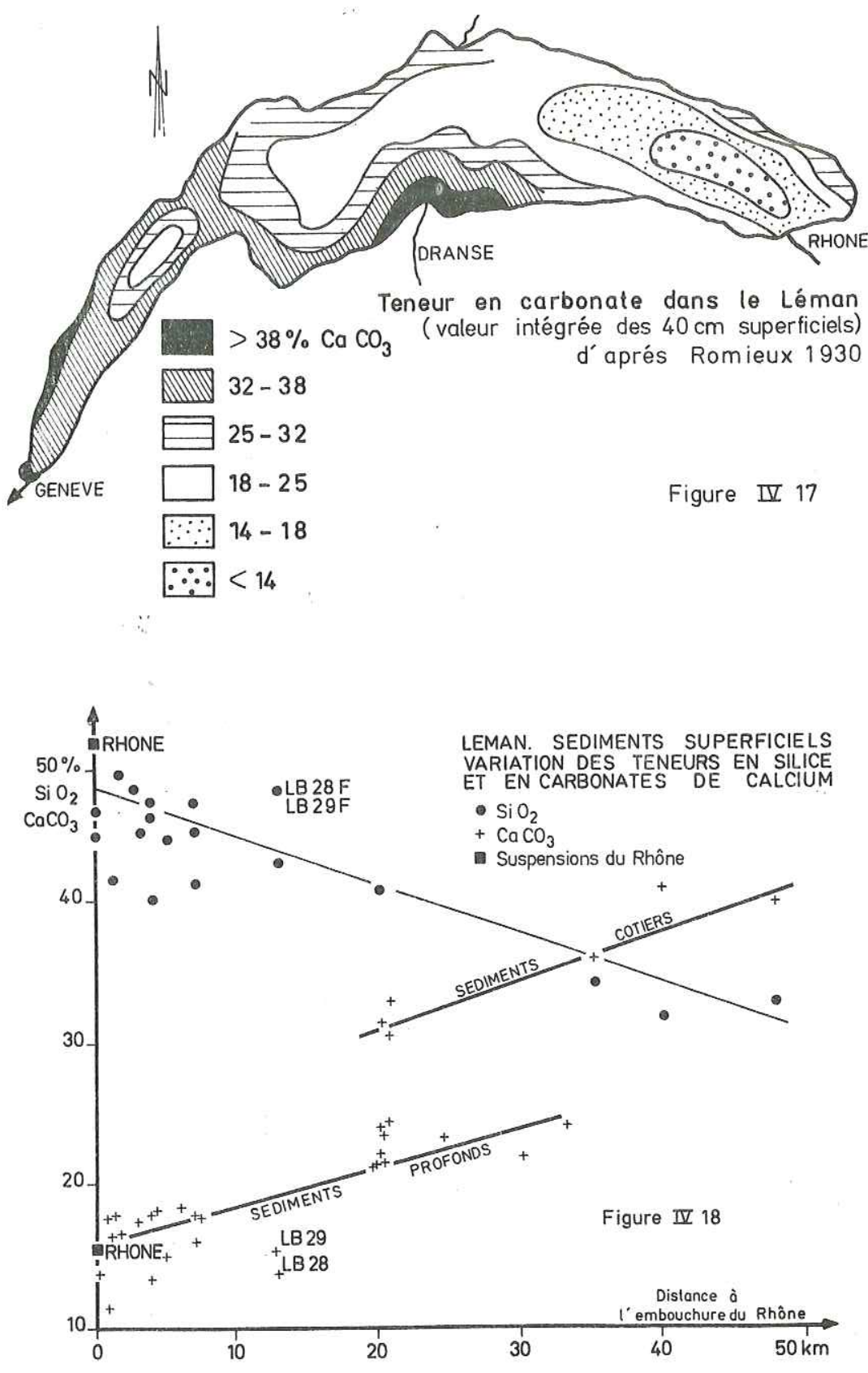

tous ces rapports varient du simple au quadruple environ, ce qui correspond aussi à la variation des carbonates dans les échantílions ana1ysés.

Notons que le magnésium n'est pas du tout lié au calcium, le rapport $\mathrm{Ca} / \mathrm{Mg}$ varie de 2,3 dans 1e Haut Lac à 11,8 dans le Petit Lac : la précipitation des carbonates est essentiellement calcique. De plus il faut remarquer qu'aucun élément ne semble suivre l'évolution du calcium, cet élément est done le seul à être précipité.

En comparant avec les analyses d'argíles, et des autres éléments constituant 1a vase, données par Debyser (1959) nous pourrons attribuer une origine probable à chaque élément. Le rapport $\mathrm{Al} / \mathrm{Fe}$ dans les sédiments du Léman varie de 2,44 à 3,66 avec une valeur moyenne de 2,81 ce fait montre que le fer est sans doute plus lié aux argiles (illite $\mathrm{Al} / \mathrm{Fe}=2$ et chlorite $\mathrm{Al} / \mathrm{Fe}=4$ ) qu'à des sulfures (on aurait alors une variation indépendante de 1'aluminium). La pyrite est en effet signalée par Monod (1956) mais en très faible quantîté. Sur le Bodensee, très analogue au Léman, les teneurs en pyrite sont en général inférieures à 0,2\% (Mül1er, 1967).

Le rapport $\mathrm{SiO}_{2} / \mathrm{Al}_{2} \mathrm{O}_{3}$ varie entre 3,7 et 4,5 , il est nettement supérieur à celuî des argìles (kaolininte $\mathrm{SiO}_{2} / \mathrm{Al}_{2} \mathrm{O}_{3}=1,3$; illite $\mathrm{SiO}_{2} / \mathrm{Al}_{2} \mathrm{O}_{3}=1,95$, chlorite $\left.\mathrm{SiO}_{2} / \mathrm{Al}_{2} \mathrm{O}_{3}=2,15\right)$ et des feldspaths qui sont d'aîlleurs en faîbles quantités dans les apports. Il y a done des particules très fines de silice de l'ordre du micron qui constituent une part non néglìgeable du sédiment. Ces particules peuvent être soit du quartz très fin soit des squelettes de diatomées qui sont une part importante du plancton.

Le phosphore et le manganèse ont un comportement géochimique tout à fait différent : les rapports $\mathrm{P}_{2} \mathrm{O}_{5} / \mathrm{SiO}_{2}$ varient du simple au double et $\mathrm{MnO} / \mathrm{SiO}_{2}$ du simple au quadruple, de plus cette variation n'est pas du tout régulière et n'est pas liée à la concentration 
de $\mathrm{SiO}_{2}$ comme les autres rapports, quî̀ dểcroìssent régulièrement avec la silice. Nous n'avons pu corréler le phosphore nî avec le calcium, ní avec la matière organique (perte au feu à $600^{\circ} \mathrm{C}$ ) et à part quelques valeurs près du Rhône, les teneurs sont presque constantes autour de $0,20 \%$. Signalons toutefoìs qu'aux embouchures des riviēres très polluées, cette teneur peut être beaucoup plus élevée et Monod (1956) cite des concentrations allant jusqu'à 1,20\% en face du Flon mais ces fortes valeurs décroissent rapidement puisque la valeur moyenne à $150 \mathrm{~m}$ de 1 'embouchure n'est plus que de $0,23 \%$. Le manganèse est l'élément le plus variable dans nos analyses: les concentrations vont de $0,06 \%$ à $0,49 \%$ (tableau $\mathrm{IV}_{4}$ ). On saít depuís quelques années qu'il exîste également dans les lacs des modules de manganèse ; Gorham et Swaine (1965) signalent des augmentations brusques de Mn dans les sêdiments du lac Windermere en GrandeBretagne, et Semenovich (1966) faît les mêmes remarques à propos du lac Ladoga en U.R.S.S., ces deux lacs étant très différents du Léman. La gếochimie du manganèse est donc un problème général, des études particulières seraient nécessaires pour le résoudre.

\subsection{3. - La précipitation des carbonates.}

Nous disposons de deux travaux très intéressants : celui de Romieux (1925) sur le Léman et celui de Portner (1951) beaucoup plus complet sur le lac de Neuchatel. D'après Romieux la teneur en $\mathrm{CaCO}_{3}$ dans le Petít Lac peut dépasser $70 \%$ dans les zones cotières, Joukowski et Buffle (1938) citent des valeurs identiques pour ce qu'ils nomment la "craie lacustre". Comme nous 1 'avons remarqué la teneur en $\mathrm{CaCO}_{3}$ est beaucoup plus forte (figure $\mathrm{IV}_{17}$ ), à distance égale du Rhône, dans les zones peu profondes et les fortes teneurs du Petit Lac viennent renforcer cette affirmation. Il semble donc que, si la précipitation des carbonates a lîeu sur tout le lac comme nous l'avons vu au chapitre III, il y a une dissolution importante de ceux-ci dans les zones profondes en raison de la température et du pH plus faibles. Cette redissolution a été nettement mise en évidence par Portner pour le lac de Neuchate1. Dans le Haut-Lac cette sédîmentation biochimique est en plus masquée par les apports terrìgènes beaucoup plus importants. Au contraire dans le Petit Lac, dont la profondeur moyenne est de $40 \mathrm{~m}$ et où 1 'hypolimnion froîd est beaucoup plus rêduit que dans le Grand Lac,cette précîpitatìon est prépondérante, les apports détritiques étant très réduits.

Comme nous venons de le voîr seul le calcîum est précipité, le magnésium évoluant de façon ìnverse comme le reste de la fractíon détritique. Ce phénomène n'a pas été remarqué dans le Bodensee où Müller (1967) note dans 1'épilimnion en Août 1965 un affaiblissement en magnésium aussì important que celuì du calcîum.

Nous avons essayé au chapitre III de mettre en évidence 1a précipitation par des billans de calcîum et de bicarbonates. Notre bilan portait sur un an et, vu les erreurs commises, nous n'avons pu conclure bien que le bilan laissaît prévoîr une précipitation d'environ 150000 t de $\mathrm{CaCO}_{3}$ par an. Balavoỉne (1939) considérant que la couche de 0 à $25 \mathrm{~m}$ perdait $10 \mathrm{mg} / 1$ de $\mathrm{CaCO}_{3}$ de Mars à Septembre, annonce une précîpitation de $145000 \mathrm{t}$ de $\mathrm{CaCO}_{3}$, mais ce calcul est critiqué par Portner parce que ne tenant pas compte des apports des rivières et des sorties à Genève pendant la période considérée. Nous avons essayé à nouveau de faire un bilan plus précis entre Septembre 1968, Mars 1969 et Septembre 1969. Nous avons évalué à ces périodes 1a teneur moyenne en $\mathrm{Ca}^{++}$et $\mathrm{HCO}_{3}{ }^{-}$du 1 ac pour chaque couche (supérieure, moyenne, intermédiaire et profonde) définie au précédent chapitre. Puis nous avons calculé les apports en tenant compte de la variation des éléments en fonction du débit. Le bilan des entrées et des sorties s'est alors trouvé équilibré, compte tenu de la variation de niveau du lac (en Mars le niveau est $70 \mathrm{~cm}$ plus bas qu'en Septembre) et des erreurs commises. Seules les variations de teneurs du lac étaient donc à considérer : entre Septembre 1968 et Mars 1969 le Léman s'est enrichi de 700000 t de $\mathrm{CaCO}_{3}$, puis de Mars 1969 à Septembre 1969 
11 en a perdu $500000 \mathrm{t}$. Ces quantìtés consìdérables peuvent paraître significatives mais elles sont en fait à considêrer avec beaucoup de prudence, étant de 1 'ordre de 3 à $5 \%$ de la quantité totale de carbonates dissous dans le Léman.

Puisque le magnésìum ne prếcìpite pas, nous avons aussỉ comparé les rapports $\mathrm{Ca} / \mathrm{Mg}$ des apports au Léman $(8,3)$ et de la sortie à Genève $(8,0)$. Là aussî compte tenu des erreurs commises nous ne pouvons pas conclure même sì la variation est dans le sens prévu.

Portner (1951) a effectué dans le lac de Neuchatel un bilan analogue en tenant compte des apports et des sorties, il trouve $90000 \mathrm{t}$ de $\mathrm{CaCO}_{3}$ précipité par an, maìs quand on consîdère qu'il y a dans ce $1 \mathrm{ac} 1,7 \cdot 10^{6} \mathrm{t}$ de $\mathrm{CaCO}_{3}$ dissout, ce bilan n'est plus beaucoup significatif, pourtant les sédiments du lac de Neuchatel sont essentiellement calcaires ( $67 \%$ de $\mathrm{CaCO}_{3}$ en moyenne). La sédimentation calcaire est donc lente et trop faible pour pouvoir être mise en évidence actuellement par des bilans d'éléments dissous. Seules les analyses chimiques des sédiments la mettent nettement en évidence.

\subsection{4. - Premières conclusions sur la nature chimique des} sédiments du Léman.

Il y a done partout dans le Léman le même stock détritique constitué essentiellement par des argiles avec un peu de calcite et de la silice très fine vers l'embouchure du Rhône et dans les zones profondes, ce stock constitue la majeure partie du sédiment. Dans les zones cotières la précipitation du carbonate de calcium devient importante car il n'y a pas redissolution du précipité dans les eaur profondes. Dans le Petit Lac ce phénomène est prépondérant : les sédiments vont des "vases calcaires" (50\% de $\mathrm{CaCO}_{3}$ ) aux "craies lacustres" (75 \% de $\mathrm{CaCO}_{3}$ ).

Parmi les éléments majeurs, $\mathrm{Al}_{2} \mathrm{O}_{3}, \mathrm{~F}_{2} \mathrm{O}_{3}, \mathrm{Na}_{2} \mathrm{O}, \mathrm{K}_{2} \mathrm{O}$ et une partie de $\mathrm{SiO}_{2}$ doivent provenir des argiles (illite, chlorite) et d'autres alumino-silicates. Une partie de la silice est sous forme de quartz très fin ou provient de test de diatomées, Mgo provient sans doute des argiles (chlorite). Si le titane doit provenir des argiles, la constance des teneurs en phosphore et les variations irrégulières du manganèse posent des problèmes géochimiques non encore résolus.

\section{9. - Sédimentation comparée du Léman et des autres lacs.}

Nous comparerons le Léman à deux lacs bien connus : 1e Bodensee (travaux de Müller et a1) et le lac de Neuchate1 (travaux de Portner). Nous avons déjà vu que les sêdiments du lac de Neuchatel sont essentiellement calcaires $\left(67 \%\right.$ de $\mathrm{CaCO}_{3}$ ) et d'origine biochimique. Le Petit Lac se rapproche donc nettement du lac de Neuchate1. Dans 1e Bodensee Mü1ler (1966 b) remarque les phẻnomènes suivants :

- sédimentation détrîtique près du Rhin et dans les zones profondes. La taille des particules diminue rapidement lorsqu'on s'éloigne du Rhin.

- teneur en carbonates plus fortes dans les zones cotières (30\% à $40 \%$ ) et dans le Gnadensee (supérieure parfois à $70 \%$ ), que dans les zones centrales profondes ( $20 \%$ à $30 \%$ ).

Une fois encore nous remarquons la similitude des deux lacs, mais Müller ne mentionne pas le rôle du canyon du Rhîn connu pourtant depuis presque un siècle : en effet le Rhin a été canalisé en 1900 et actuellement il semble que le canyon ne fonctionne plus, les débits du Vieux Rhin étant trop faibles. Ce canyon a dû pourtant jouer un rôle identique à celui du Rhône dans le Léman : il est visible jusqu'à la profondeur de $205 \mathrm{~m}$, sur le premier kilomètre sa pente est de $8 \%$ et sa profondeur atteint $70 \mathrm{~m}$. Il n'existe pas à la nouvelle embouchure du Rhin à Fussach de phénomène analogue. 
Zender (1908) avait judicieusement classé les lacs subalpins en trois catégories: les lacs calcaires (Neuchater, Bienne), les lacs mixtes (Léman, Bodensee, Quatre-Cantons) et les lacs silicatés (Majeur, Côme, Lugano), nos travaux comme ceux de Müller et de Pörtner confirment cette distinction.

\subsection{0. - Taux de sédimentation dans le Léman.}

Nous avons vu 1'hétérogénéîté des vases du Léman tant au point de vue physique que chimique, il sera donc diffìcile à priori de définir un taux de sédimentation : chaque zone aura un taux correspondant à son mode de sédimentation. Comme Serruya (1969) nous préférons exprimer le taux de sédimentation en $\mathrm{mg} / \mathrm{cm}^{2} / \mathrm{an}$ plutôt qu en mm/an, notion plus pratique mais fonction de 1 a teneur en eau et de la compaction du sédiment.

D'après les données palynologîques, C. Serruya donne les résultats suivants pour des zones de $100 \mathrm{~m}$ de fond dans le Grand Lac.

Subatlantique - subboréal : $65 \mathrm{mg} / \mathrm{an} / \mathrm{cm}^{2}$

$\begin{array}{ll}\text { Atlantique } & : 48 \mathrm{mg} / \mathrm{an} / \mathrm{cm}^{2} \\ \text { Boreal } & : 90 \mathrm{mg} / \mathrm{an} / \mathrm{cm}^{2} .\end{array}$

De plus une carotte a pu être datée au $\mathrm{C}^{14}$ en face de Lausanne à faible profondeur, on aurait dans cette zone une sédimentation de $1 \mathrm{~mm} / a n$ (Serruya, 1969). Dans notre étude de la crue de la Dranse (Hubert et a1, 1969), nous avons estimé la sẻdimentation due à 1a crue à $1 \mathrm{~mm}$ de sédiment $\mathrm{sec}$, ce qui montre bien l'importance de ce phénomènes. On peut également se baser sur les varves : on a alors une sédimentation de plusieurs cm/an dans la zone proche du Rhône et de l'ordre du mm/an dans la plaine centrale mais ces estimations sont basées sur la périodicité annuelle des varves,ce qui n'est pas encore établi avec certitude.
Nous avons signalé le morceau de scorie de charbon trouvê au niveau $132 \mathrm{~cm}$ dans 1 a carotte $\mathrm{LZ}_{8}$. Cette scorie ne peut provenir que d'un bateau à vapeur or cet endroît n'a été désservì par de tels bateaux que de 1854 à 1964 , ce quî donne un taux de sêdimentation dans cette région face à St-Gingolph de $0,8 \mathrm{~cm} /$ an à 2 ou $3 \mathrm{~cm} / \mathrm{an}$. Mais si 1 'on tient compte des niveaux de turbidites mis en place d'un seul coup, on arrive à une valeur moyenne de 2 à $3 \mathrm{~cm} / \mathrm{an}$ pour la sédimentation régulière, ce qui correspond aux varves de cette carotte.

Au centre du lac deux déterminations du taux de sédimentation par les radionuclides artificiels ont été réalisées. La première en 1968 par J.M. Martin du laboratoire de Gêologie Dynamique de Paris, porte sur 1 e $\mathrm{Cs}^{137}$ : 1e maximum d'activité de 1963 a été retrouvé au niveau 2 à $4 \mathrm{~cm}$, on aurait donc un dépôt de $0,5 \mathrm{~cm} / \mathrm{an}$ environ. La deuxième étude par le Docteur D. Lal du Tata Institute de Bombay porte sur le $\mathrm{Fe}^{55}$ et 1 e $\mathrm{Pb}^{210}$, le premier élément donne un taux inférieur à $0,1 \mathrm{~cm} / \mathrm{an}$ et le deuxième un taux de $0,4 \mathrm{~cm} / \mathrm{an}$. Cette dernière valeur qui corrobore celle de Martin semble donc la plus probable.

Toutes ces déterminations n'ont que des valeurs ponctue1les. Nous avons estimé les apports annuels au Léman entre 7 et 9 millions de t/an en grande majorité des sédiments fins, ce qui correspond à une valeur moyenne de $1,4 \mathrm{~g} / \mathrm{cm}^{2} / \mathrm{an}$ sur tout le Léman, ou $\mathrm{d}^{\prime} 1,6 \mathrm{~g} / \mathrm{cm}^{2} /$ an si on excepte le Petit Lac.

Cette valeur est du même ordre de celle qu'on peut calculer d'après les données de Müller pour le Bodensee (Obersee seulement) $0,60 \mathrm{~g} / \mathrm{cm}^{2} / \mathrm{an}$. Ces taux sont considérables par rapport à ceux des lacs de Neuchatel ( $65 \mathrm{mg} / \mathrm{cm}^{2} / \mathrm{an}$, calculé d'après Portner), ou de Ladoga $\left(6,5 \mathrm{mg} / \mathrm{cm}^{2} /\right.$ an d'après Semenovich). 


\section{C - BILAN SEDIMENTAIRE DU BASSIN VERSANT DU LEMAN}

ET EROSION SPECIFIQUE TOTALE DES ELEMENTS MAJEURS.

\subsection{1. - Composition chimique des suspensions lacustres}

Sur la vingtaine de prélèvements de gros volume (50 1 à 100 1) réalisés dans le Lêman et à Genève, seule une douzaỉne était suffisamment chargée pour effectuer une analyse chîmìque totale. Les moyennes et les extrêmes sont données dans le tableau $I_{4}$. On note tout de suite une grande variabilité des éléments, ill n'a pas été possible de corréler les analyses avec les saisons, avec 1'emplacement des prélèvements ou leurs profondeurs, en raison du trop petit nombre de données. L'étude des suspensions lacustres ou marines (seston) est très particulière et 1 'analyse chìmique doit aussi tenir compte de leur nature minérale ou organique. On peut noter cependant $1 \mathrm{a}$ forte teneur générale en $\mathrm{P}_{2} \mathrm{O}_{5}$ qui est due au plancton riche en phosphore. A part cet élément les suspensions lacustres ont en général des concentrations intermédiaires entre celles du Rhône et celles de la Dranse.

Nous avons déjà vu que la teneur moyenne en suspension des eaux de sortie à Genève était très faible : $4 \mathrm{mg} / 1$ d'après les données de 1'Office Fédéral de 1'Economie Hydraulique, ce qui représente $30000 \mathrm{t}$ de suspensions par an. Par rapport aux apports cette valeur est très faible, on peut donc considérer que tout ce qui arrive en suspension au Léman, s'y dépose. L'érosion spécifique du bassin versant du Léman à la sortie du lac est done essentiellement chimique. (voir tableau $\operatorname{III}_{14}$ ).

\subsection{2. - Erosions détritiques et chimiques comparées par éléments.}

Nous pouvons maintenant comparer les érosions détritiques et chimiques des éléments suivants $: \mathrm{SiO}_{2}, \mathrm{Ca}, \mathrm{Mg}, \mathrm{Na}, \mathrm{K}$, ainsi que pour 1 e $\mathrm{CO}_{2}$ et 1 e $\mathrm{P}$. Nous avons exprimé les apports en suspensions (fraction minérale seulement) en composittion élémentaîre et non pas en oxydes -sauf pour la silice et 1 e $\mathrm{CO}_{2}$ - pour pouvoir les comparer aux apports en solution. La plupart des éléments de ce bilan proviennent des phénomènes naturels d'êrosion chìmique et détritique, sauf le phosphore. Cet élément, que nous n'avons pas dosé dans les eaux, est cependant bien connu par les travaux de la Commission de Protection du Léman, les valeurs fournies ici concernent le phosphore total (phosphore soluble et phosphore organique). En raison des nombreuses sources artificielles de phosphore (excrétions, détritus ménagers, détergents, engraîs, etc...) le phosphore nature1 d'origine minérale des eaux ne représente plus qu'une partie de phosphore dosé (1'étude quantitative des provenances reste encore à faire). 
TABLEAU IV 8 : EROSIONS CHIMIQUES ET DETRITTQUES COMPAREES PAR ELEMENT.

Sur 1a base de $6.10^{6} \mathrm{t} /$ an d'apports minéraux en suspension pour le Rhône et de $0,5.10^{6} \mathrm{t} / \mathrm{an}$ pour 1a Dranse.

\begin{tabular}{|c|c|c|c|c|c|c|c|c|c|c|c|}
\hline \multirow{2}{*}{$\begin{array}{l} \\
\vdots \\
\vdots \\
\vdots\end{array}$} & \multicolumn{5}{|c|}{ Rhồne } & \multicolumn{6}{|c|}{ Dranse } \\
\hline & $\begin{array}{l}\text { A } \\
\vdots \text { dét } \\
\vdots\end{array}$ & $\begin{array}{l}\text { Apports } \\
\text { Eritiques } \\
10^{3} t\end{array}$ & & $\begin{array}{l}\text { Erosion } \\
\text { létritique } \\
\mathrm{t} / \mathrm{km}^{2} / \mathrm{an}\end{array}$ & $\begin{array}{l}\text { Erosion } \\
\text { chimique } \\
\text { t/ } / \mathrm{km}^{2} / \mathrm{ar}\end{array}$ & $\begin{array}{c}\vdots \\
\vdots \\
\vdots\end{array}$ & $\begin{array}{l}\text { Apports } \\
\text { létritiques } \\
10^{3} \mathrm{t}\end{array}$ & & $\begin{array}{l}\text { Erosion } \\
\text { lêtritique } \\
\mathrm{t} / \mathrm{km}^{2} / \mathrm{an}\end{array}$ & $\begin{array}{l}\text { Erosion } \\
\vdots \mathrm{chimique} \\
\mathrm{t} / \mathrm{km}^{2} / \mathrm{an}\end{array}$ & $\begin{array}{l}\vdots \\
\vdots \\
n !\end{array}$ \\
\hline $\mathrm{SiO}_{2}$ & $!$ & 3150 & $!$ & 600 & 3,2 & $!$ & 210 & $!$ & 380 & 5,0 & \\
\hline$! \mathrm{Ca}$ & $!$ & 310 & $!$ & 60 & $!$ & $!$ & 68 & $!$ & 125 & $!$ & \\
\hline $\mathrm{Mg}$ & $!$ & 190 & $!$ & 36,5 & 5,4 & $!$ & 10,5 & $!$ & 19 & 12 & $!$ \\
\hline ! $\mathrm{Na}$ & $!$ & 80 & $!$ & 15,5 & 3,3 & $!$ & 2,15 & $!$ & 3,9 & $! \quad 2,3$ & $!$ \\
\hline $\mathrm{k}$ & $!$ & 165 & $!$ & 32 & 1,35 & $!$ & 10,5 & $!$ & 19 & $\vdots 1,6$ & $\vdots$ \\
\hline $\mathrm{CO}_{2}$ & $!$ & 460 & $!$ & 88 & $! 65$ & $!$ & 84 & $!$ & 150 & $! 195$ & $!$ \\
\hline ! & $!$ & 7,2 & $!$ & 1,38 & 0,093 & 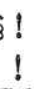 & 0,48 & $!$ & 0,87 & $0,023^{\S}$ & $\$ !$ \\
\hline
\end{tabular}

Phosphore total (d'après Burkard, Commission de Protection du Léman).

Il ressort de ces valeurs les faits suivants :

- Environ $99 \%$ de la silice transportée par les rivières provient de l'érosion détritique.

- Le calcium et le $\mathrm{CO}_{2}$ proviennent,pour les deux rivières à part égale sous forme dissoute et en suspensions.

- Pour le Rhône le magnésium, sodium et potassium proviennent essentiellement de l'érosion détritique, par contre pour la Dranse un tiers du magnésium et du sodium provient des dissolutions.

- Enfin la quantité de phosphore amenée au Léman par dissolution est très faible par rapport aux apports minéraux, même pour des rivières déjà polluées. Ce fait doit nous amener à considérer avec prudence les bilans de phosphore, en effet une dissolution partielle, du phosphore minéral (apatite par exemple) dans les sédiments et les suspensions suffirait à égaler les apports dissous et à fausser les bilans.

Ces remarques qui confirment de facon logique ce qui est connu sur l'érosion des reliefs, permettent de chiffrer, pour un climat précis, la proportion des deux types d'érosion pour chaque élément considéré. Il manque malheureusement à ces valeurs celles des composés sulfurés. Le dosage du soufre dans les sédiments est délicat et la distinction entre soufre organique et minéral est difficile à faire. Néanmoins les teneurs très faibles en $\mathrm{SO}_{3}(0,5 \%)$ des sédiments du Léman (Monod, 1956) nous laissent à penser que le soufre doit être amené à $90 \%$ environ sous forme soluble (sulfates). 


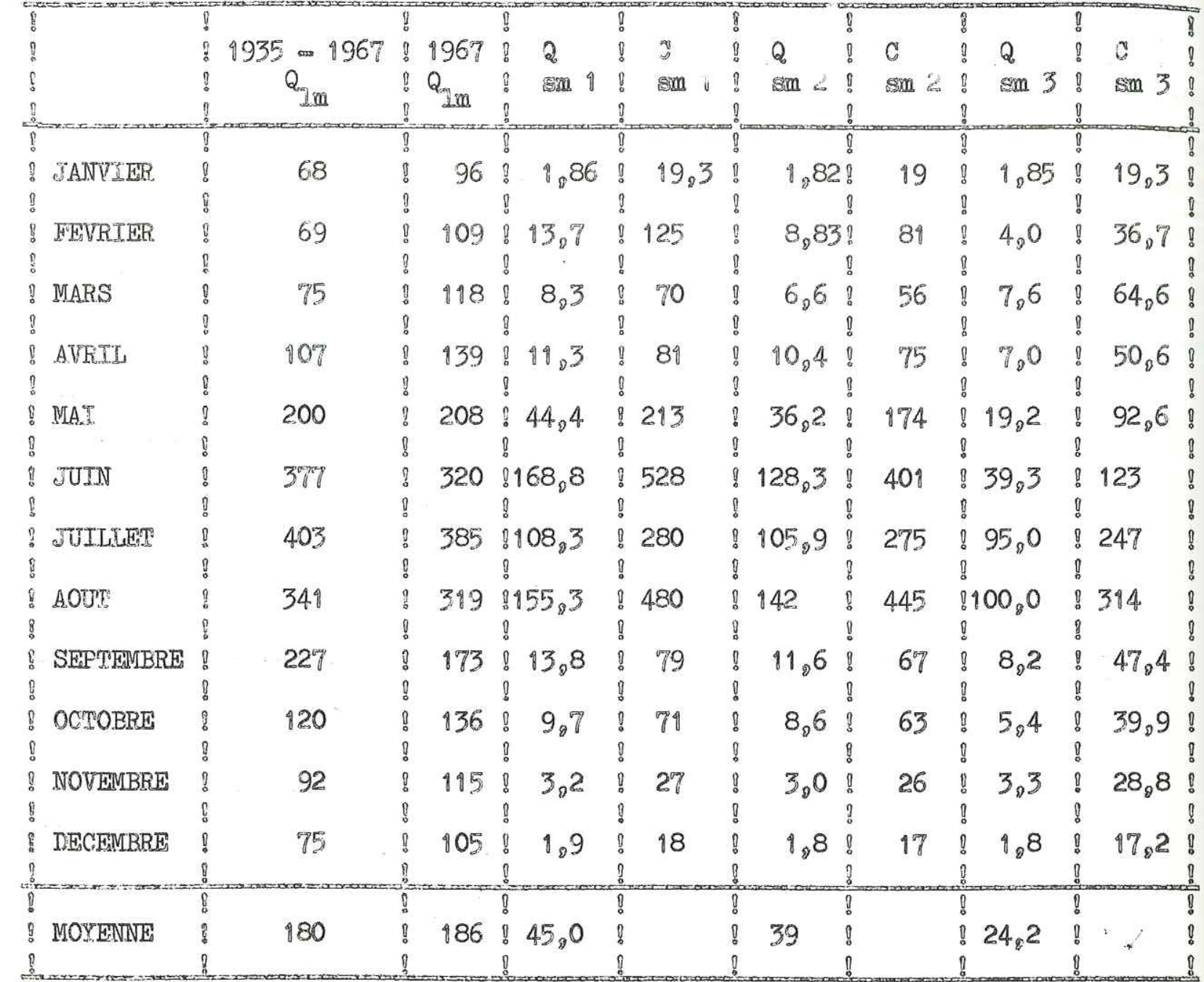

TABLEAUU IV,

DEBITS IIQUIDE ET SOLIDES EN 1967

Q1 = débitts liquides en $\mathrm{m}^{3} / \mathrm{s}$

Qs $=$ débits solides on $\mathrm{kg} / \mathrm{s}$

$\mathrm{Cr}=$ charge en suspension $\mathrm{g} / \mathrm{m}^{3}$
Thatran $\mathrm{NV}_{2}$

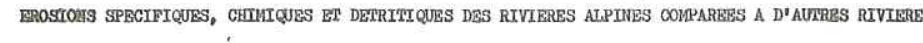

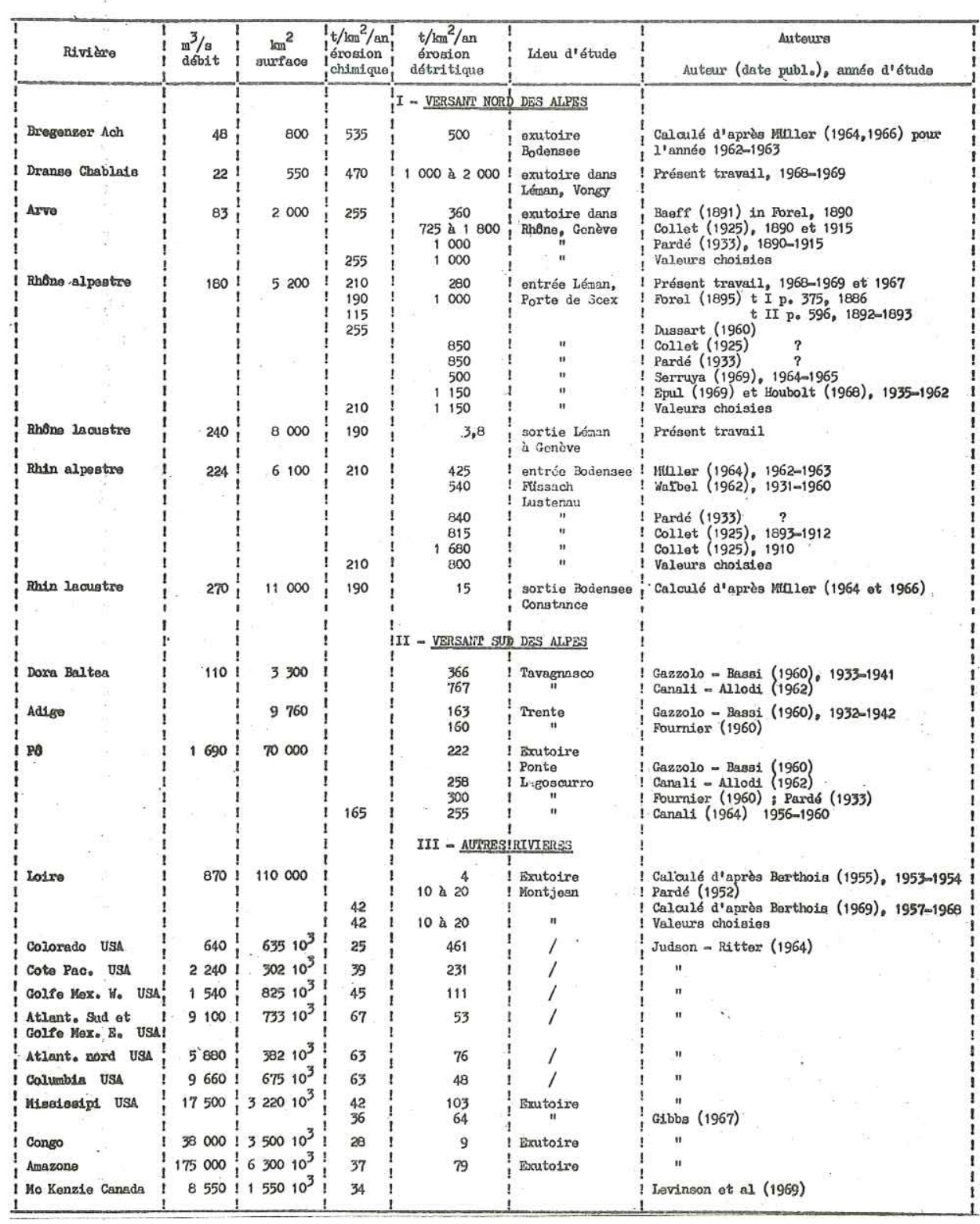



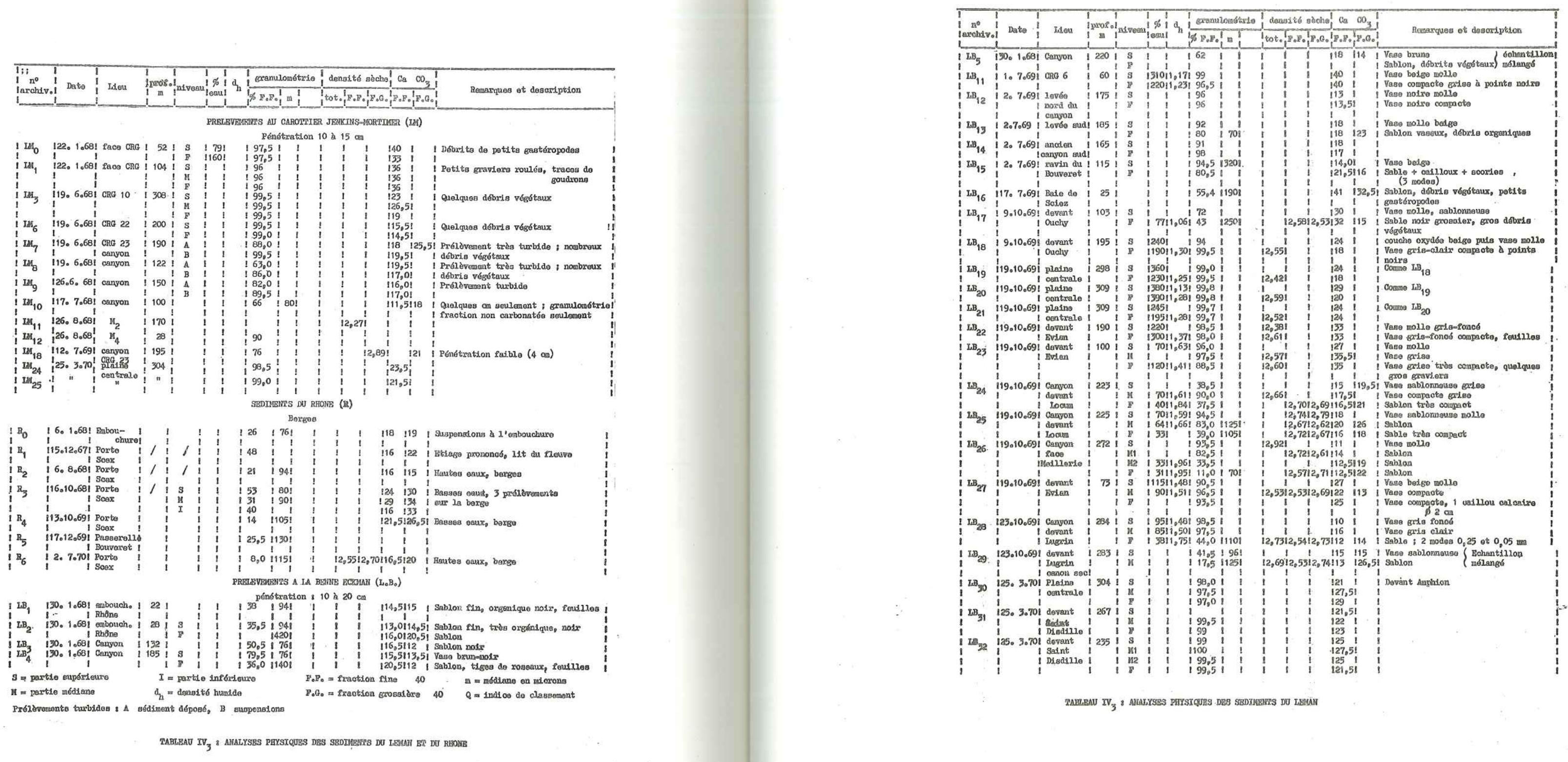


\begin{tabular}{|c|c|c|c|c|c|c|c|c|c|c|c|}
\hline & \multirow{2}{*}{\multicolumn{3}{|c|}{ RrHONE }} & \multirow{2}{*}{\multicolumn{3}{|c|}{ DRANSE }} & \multicolumn{5}{|c|}{ LAC } \\
\hline & & & & & & & \multicolumn{3}{|c|}{ Suspensions } & \multicolumn{2}{|c|}{ Sédìments } \\
\hline & $\mathrm{m}$ & $\min$ & $\max$ & $\mathrm{m}$ & $\min$ & $\max$ & m & $\min$ & $\max$ & $m ! \min !$ & 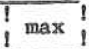 \\
\hline & & & & & 34,0 & 51,0 & 53,6 & 4, & & $43,0130,01$ & $1.51,6$ \\
\hline $0_{3}$ & & & & & & & 10,6 & & 16,5 & $11,3: 7$ & \\
\hline & & & & 08 & & & 0,124 & & & , 11510, & 10,4 \\
\hline $\mathrm{Fe}_{2} \mathrm{O}_{3}$ & 11 & & 8,21 & 4,10 & & 6,26 & 5,15 & 1,8 & 7,7 & $4,04 ! 2,86 !$ & 5,1 \\
\hline & & & & 2,51 & $\{1,95\}$ & 3,55 & 2,66 & 1,57 & 3,95 & $2,451 \quad 1,321$ & 3,5 \\
\hline $\mathrm{Na}_{2} \mathrm{O}$ & 83 & 1,08 & 2,43 & 0,58 & 0,36 & $\mid 1,39$ & 1,36 & $0,63 !$ & 2,40 & $1,1610,55 !$ & 3,0 \\
\hline cao & & 2,27 & 4,25 & 19,0 & 8,3 & 23,0 & 11,5 & 2,9 & 119,3 & $15,3 ! 11,9$ & 25,2 \\
\hline $\mathrm{m}_{80}$ &, 40 & 3,49 & 8,08 & 3,48 & 2,75 & 6,0 & 3,45 & $1,08:$ & 5,3 & $\begin{array}{llll}3,74 & 1,97 !\end{array}$ & 6,2 \\
\hline $\mathrm{TiO}_{2}$ & & & & 0,55 & & & 0,59 & & 0,79 & $0,59 ! \circ$ & \\
\hline $\mathrm{P}_{2} \mathrm{O}_{5}$ & 0,28 & 0,18 & 0,49 & 0,22 & 0,12 & 0,55 & 1,51 & $0,30 !$ & 5,25 & 0,2110 & 0,41 \\
\hline per 1 & 7,7 & 4,6 & 12,7 & 16,8 & 12,3 & 119,2 & 9,7 & $4,23 !$ & 16,7 & $17,8114,6$ & 28,1 \\
\hline erte & & & & & & & & & & $5,36 ! 3$ & 1,5 \\
\hline
\end{tabular}

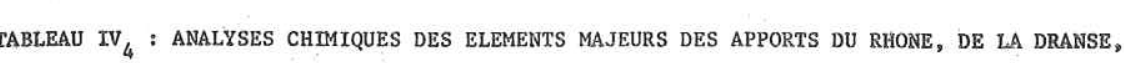
DES SUSPENSTONS ET DES SEDTMENTS DU LEMAN, VALEURS MOYENUES ET EXTRRMYS.

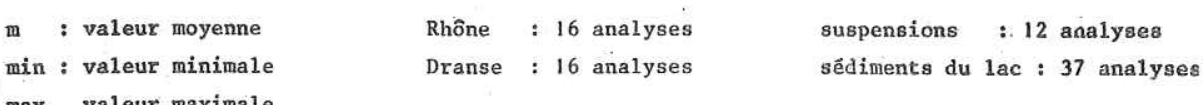

REMARQUR

analyses du Rhône, de la Dranse et des suspensions du lac sont exprimées ân $600^{\circ} \mathrm{C}$. Les analyaction minérale seulement, c'est-à-dire après calcinatio sédiment total, fraction organique comprise.

Pour les suspensions lacustres les pertes au feu théoriques ont été obtenues par différence.

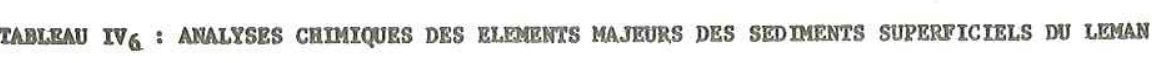

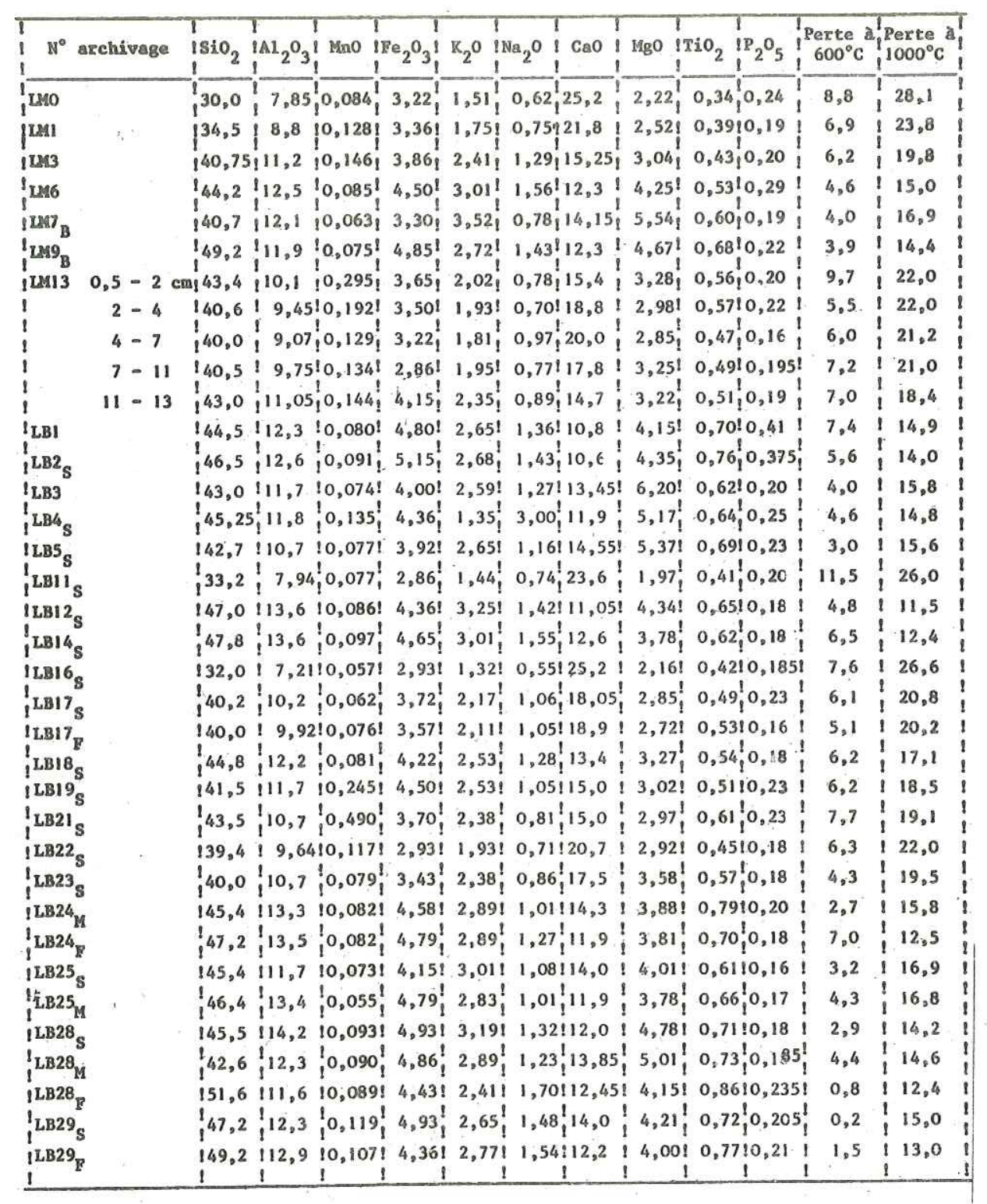



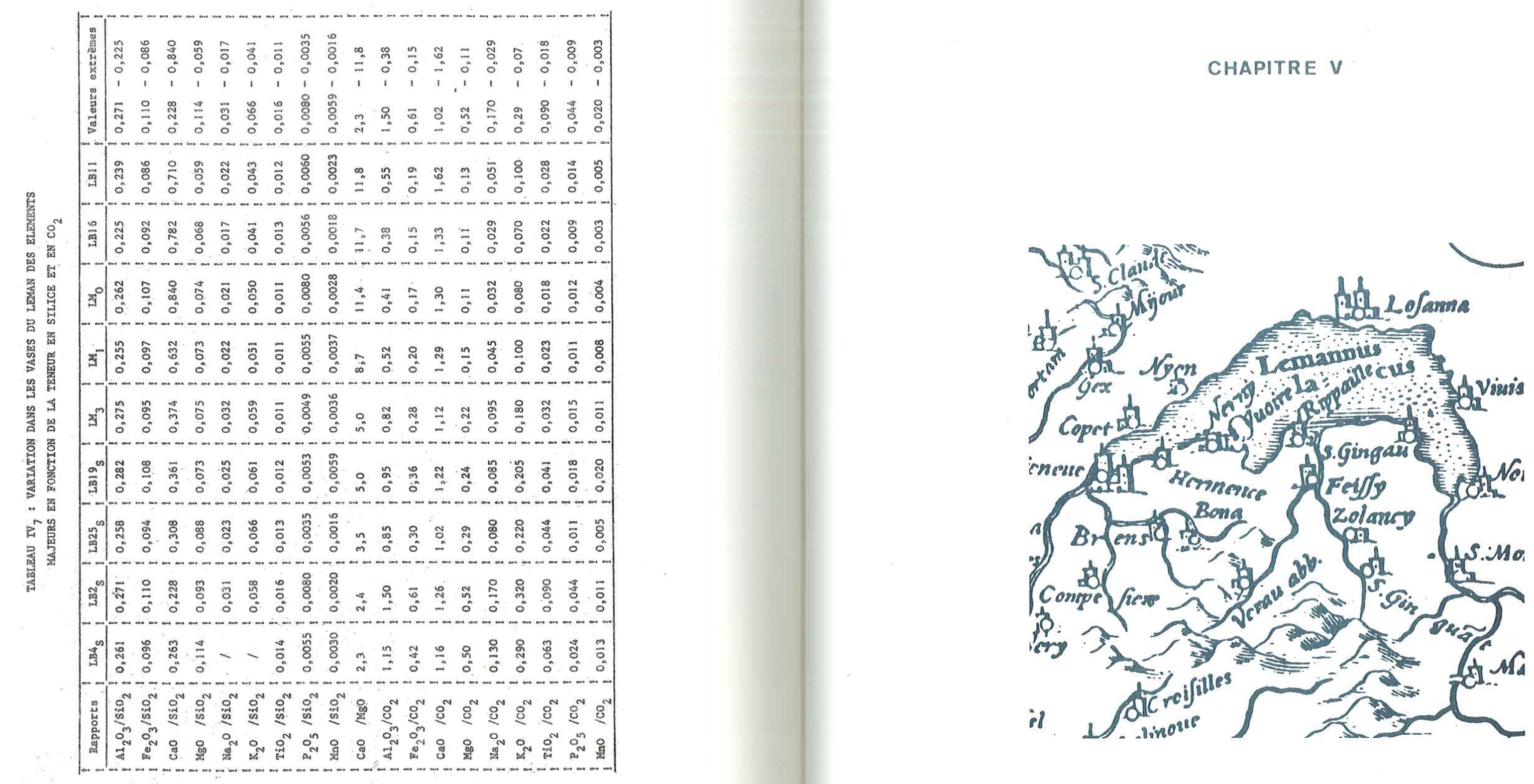

STRUCTURE ET REMPLISSAGE DE LA CUVETTE LEMANIQUe

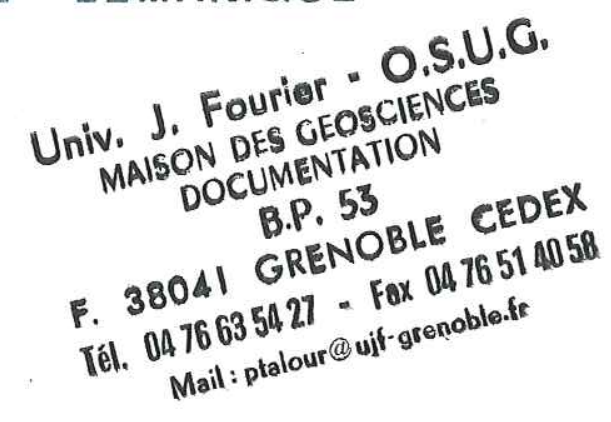




\section{1. - Nécessité d'une étude sismique.}

Plusieurs raîsons nous ont poussê à effectuer une campagne de sismique continue. Tout d'abord malgrê de nombreuses et fructueuses campagnes antêrỉeures (Wînnock, 1965; Glangeaud, 1964; Serruya, 1964; Houbolt et Jonker, 1968) quì font du Lẻman un des lacs les plus sillonnés en sondage sismìque continue (figure $V_{1}$ ), aucune estimation du remplissage sédimentaire n'avait été fournie. Or le volume des sédiments postglaciaires est une donnée qui nous permet d'estimer les vitesses de sédimentations globales pour toute une période. De plus une étude sismique supplémentaire pouvaìt préciser certains points restés obscurs notamment la structure de la côte sud du 1ac. C'est pourquoi nous avons utilisé en Avril 1969 un canon à air, méthode différente des précédentes (sparker, boomer et gaz exploder, de moins grandes pénétrations). Enfin la structure de la cuvette lémanique nous apporte de précieuses données sur l'origine et l'évolution du Léman. Après les quelques centaines de kilomètres de profils sìsmiques effectués depuis 1966 nous avons essagé de faire le point des travaux de sismique avant que d'autres méthodes soient peut-être employées.

5.2. - Situation géologique de la cuvette lémanique, éléments apportés par les études sismiques.

\subsection{1. - Structure de la région (figure $V_{2}$ )}

Le Léman est situé entre des domaines très différents : Jura au Nord-Ouest et Préalpes du Chablais et du Valais au Sud-Est. Entre 

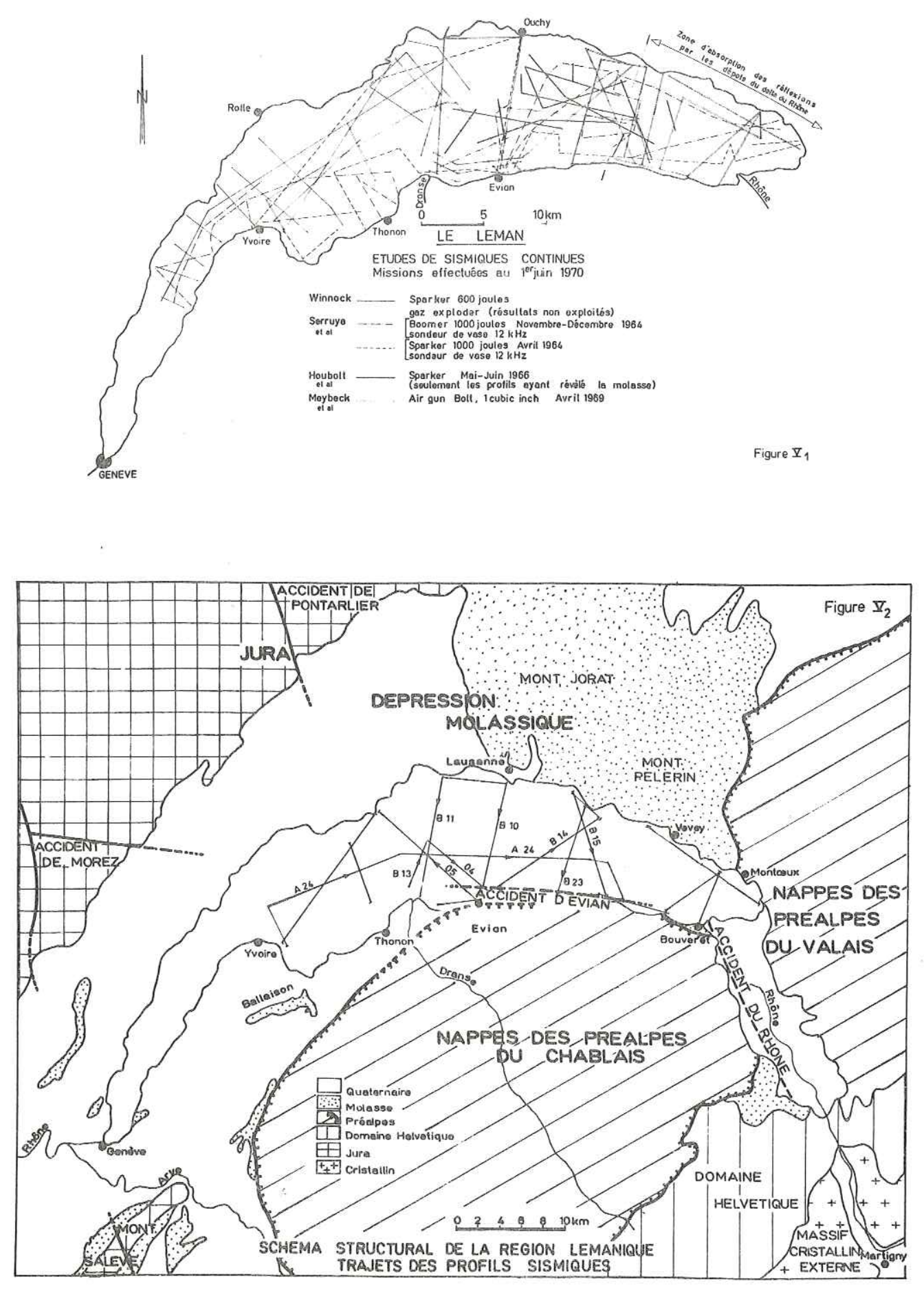

ces deux ensembles se situe le plateau molassique suìsse d'âge oligomiocène sur lequel les nappes préalpines sont venues se déverser. Une partie de ces terrains a été légèrement atteinte, on distingue alors la molasse autochtone et la molasse subalpine. Enfin au Quaternaire se sont déposées des quantités considérables de dépôts glaciaỉres et fluvio-glaciaires, souvent assez grossiers (moraînes à blocaux, conglomérats). Ces dépôts masquent la plupart des contacts anormaux et recouvrent une grande partie de la molasse notamment sur la partie méridionale du lac.

Plusieurs origines ont étê proposées pour le Lểman : Gagnebin attribue la cuvette à un creusement par les glaciers ; mais 1'origine tectonique, pressentie par Forel et exposée par Lombard (1939) semble maintenant la plus importante notamment à la suite des travaux de sismique (Serruya et al 1964) (Meybeck et al 1969).

\subsection{2. - Les horizons rencontrés en sismique continue.}

Quatre groupes d'études sismi̊ques ont étê effectués depuis 1964 (figure $V_{1}$ ), tous les auteurs ont décritt les deux horizons principaux rencontrés dans le tréfond lacustre, les sédiments lacustres et le toit de la molasse. Dans certains endroits comme la baie de Sciez ou la région d'Evian, où les terrains glaciaires affleurent, certains auteurs ont identifiê un ou plusieurs horizons glaciaires. Une quatrième unité est visible sur quelques enregistrements, il s'agit des nappes des préalpes sur la côte méridionale du lac.

\subsection{3. - Problèmes posés par l'identification des horizons.}

Un réflecteur est identifié à coup sûr quand on peut le suivre depuis un affleurement côtier, c'est le cas de la molasse qui affleure largement en Suisse et quî est bien reconnaissable par le pendage de ses couches. I1 en est de même pour le glaciaire si on peut le suivre depuis l'affleurement. Enfin, il n'y a aucun problème pour identifier les sédiments lacustres : les réflecteurs sont nets, 
$-190-$

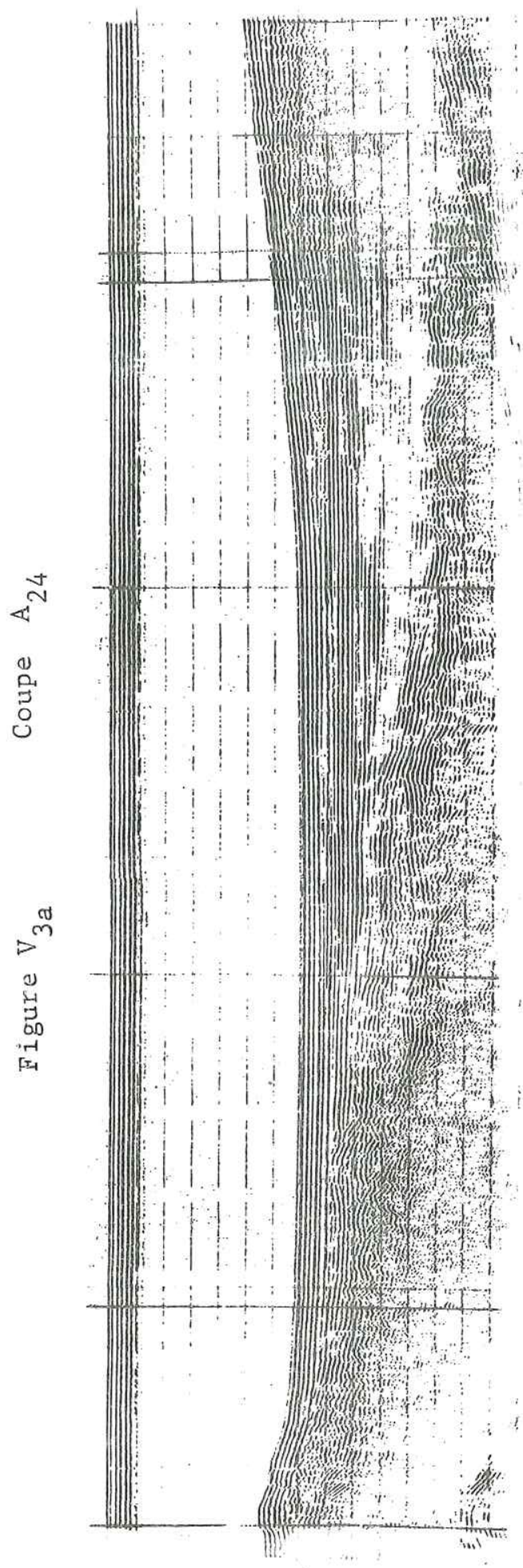

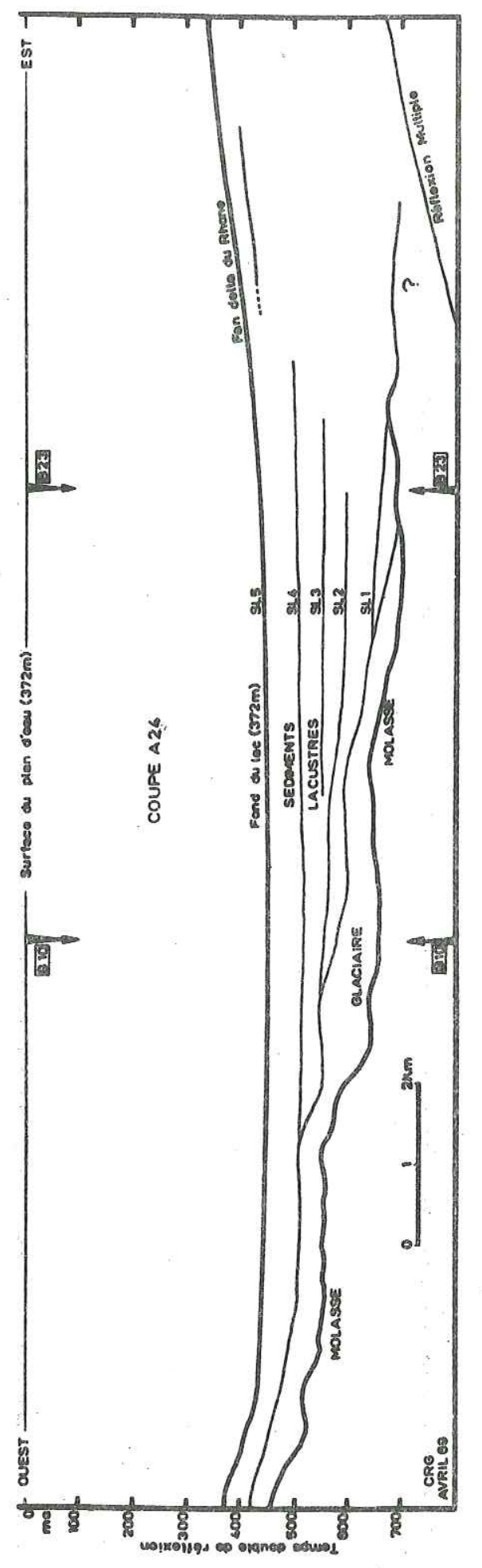

très réguliers et en général horîzontaux partîculièrement dans $1 a$ plaine centrale du lac. Néanmoins un problème important d'interprêtation se pose à l'endroît du contact du glaciaaire ${ }^{\S}$ sur la molasse. Ce contact est masqué par une forte épaisseur de sêdiments lacustres. C'est aînsì que sur la même coupe Evian-Ouchy, nous avons donné (Meybeck, Hubert, O1ive, Sìwertz et Glangeaud 1969) une interprétation tout à faît différente de celle de Serruya. Au niveau du de1ta de la Dranse les choses se compliquent encore du fait des dépôts grossiers deltai̊ques de la rivière. Enfîn plusieurs auteurs (Serruya, Houbolt) ont distingué un réflecteur intermédiaire sous les sédiments de la plaine centrale mais au-dessus de la molasse, sans 1'attribuer avec certitude au glaciaire.

Le dernier problème soulevé est celuî du R.P.E., Réflecteur Profond d'Evian, bien mis en évîdence dans notre dernière campagne, nous l'avons rapproché de la molasse (Meybeck et al 1969), sans exclure la possíbilité d'une nappe préalpine.

\section{3. - Résultats de la campagne de sismique continue d'Avril 1969.}

Cette campagne est le fruit d'un travail d'équipe qui a déjà fait l'objet d'une publication donnant les résultats dans leurs lignes principales. Nous présentons ici les résultats plus détaillés du dépouillement complet des enregistrements.

\subsection{1. - Méthode d'étude. Appareillage.}

Pour obtenir de grandes pénétrations, but de cette étude, nous avons utilisé le "canon à aîr" type Bolt du C.R.G. de Villefranche-sur-Mer, dont la chambre avait un volume d'l inch cube et qui

$\S$ Glaciaire est pris ici dans un sens général sans distinction du glaciaire et de l'interglaciaire. 

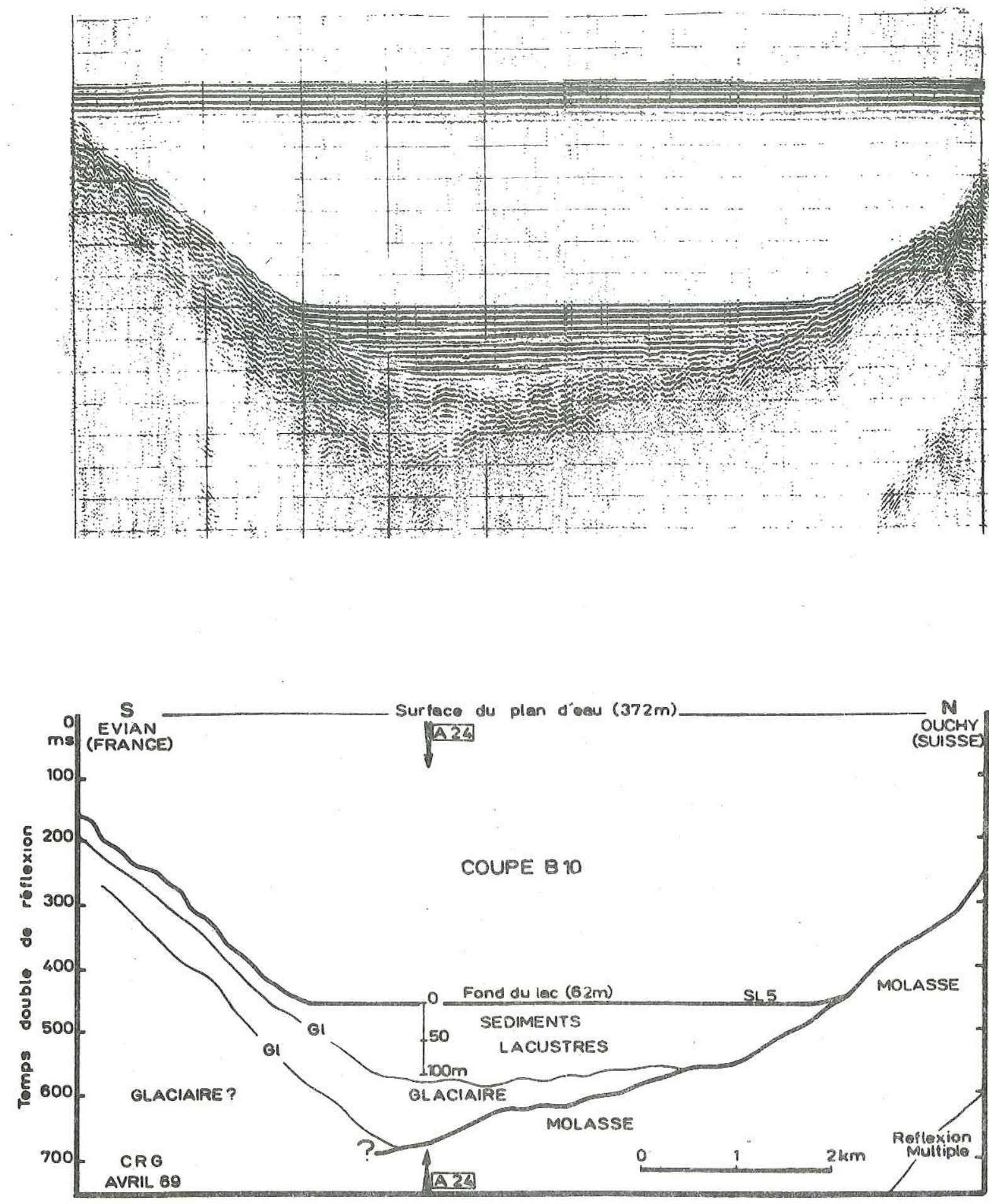

Figure $\mathrm{V}_{3} \quad$ Coupe $\mathrm{B}_{10}$ produisait, toutes les 2 secondes, une explosion à une pression de $60 \mathrm{~kg} / \mathrm{cm}^{2}$. Ce canon était remorqué à $40 \mathrm{~m}$ du bateau ("Le Vendôme") tandis que 1'"Anguille" contenant les hydrophones se trouvait à $200 \mathrm{~m}$. Des essais prélîminaîres ont montré que la vîtesse optimale du bateau était de 8 noeuds et que 1 'échelle de temps la plus appropriée était de 0 à 1 seconde. Le fîltrage élìminnant les bruits parasites a rendu les enregistrements particulièrement lisisibles $\S$ Le repérage du bateau était assuré par deux équîpes de topographes munis de théodolites et en liaison constante avec le bateau par radiotéléphones. Le bateau était repéré toutes les deux ou trois minutes, au début et à 1 a fin de chaque coupe, chaque fois enfin qu'il convenait de marquer le passage d'une structure intêressante.

Le trajet des coupes fut déterminé en fonction des résultats antérieurs de sismique, de la géologie des rives suisses et françaises et des accidents du relief sous lacustre. Deux cents kilomètres de profils de grande pénétration ont été aînsi réalisés. Nous avons réalisé un certain nombres de transversales et d'obliques qui ont toutes été recoupées par 1 a grande longitudinale $\mathrm{A}_{24}$ de $32 \mathrm{~km}$ (figure $\mathrm{V}_{3}$ ); 1 'expérience a montré que les petits trajets étaient difficilement utilisables de même que les parties terminales des coupes où les hyperboles dûes à la rêfraction sur les pentes masquaient les réflecteurs. Ce fut notamment le cas près des côtes en face d'Evian, nous avons alors effectué des trajets parallèles à celles-ci qui se révèlèrent extrêmement ìnstructifs.

Le dépouillement des enregistrements a commencé par la mise en place des coupes les unes par rapport aux autres puis par 1'identification des réflecteurs possibles sur chaque coupe. La vision globale des réflecteurs sur toutes les coupes nous a alors permis

$\S$ Je tiens à remercier Jean Petit du C.R.G. de Villefranche-sur-Mer qui a assuré toute la partie technique de cette campagne. 
$-194-$
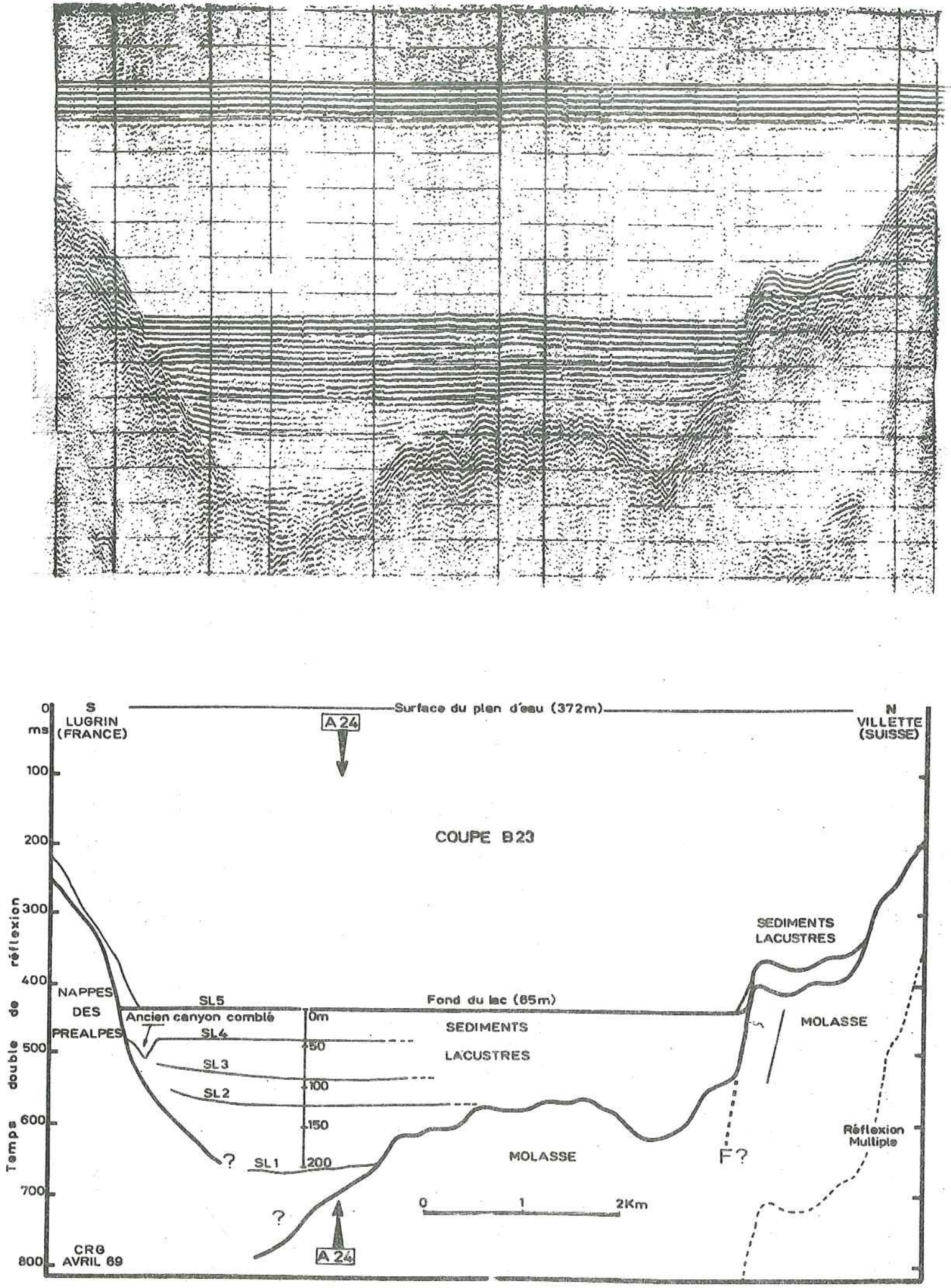

Figure V

Coupe $\mathrm{B}_{23}$ d'éliminer certaines réflexions secondaîres pour ne garder que les principales. Néanmoins dans certaînes zones les réflecteurs peuvent être mal déterminés du faît des très nombreuses rêfractions (cas de la coupe A24 depuis Yvoîre jusqu'au large de 1a Dranse). Les fonds étant souvent peu profonds et durs le dépouîliement a quelquefois été gêné en raison de l'onde dirrecte ou "bu1le" et des "multiples" (bien visibles notamment dans la région du delta de la Dranse).

L'interprétation, facilittée par la qualité de l'enregistrement, s'est faite surtout d'après le recalage sur les affleurements à terre et aussi grâce aux multiples travaux antérieurs. Enfin signalons qu'à 1'Est d'une transversale Rivaz-Locum les dépôts de1taïques du Rhône absorbent pratiquement toute l'énergie du canon à air, aucune réflexion profonde n'est plus alors visible.

Pour tenter de restituer les horizons à leur profondeur réelle nous avons choisi les vìtesses suivantes, gênéralement adoptées dans les études semblables : sêdìments lacustres $1650 \mathrm{~m} / \mathrm{s}$, dépôts glaciaires $2000 \mathrm{~m} / \mathrm{s}$. En 1 'absence de mesures directes par sismique réfraction ces valeurs ne sont qu'indicatives, aussi toutes nos coupes ont-elles été laissées en temps double de rêflexion.

\subsection{2. - Résultats.}

L'identification du glaciaire sous les sédiments de la plaine centrale a été réalisée en comparant la coupe A24 (figure $V_{3}$ ) où cet horizon est bien suivi depuis Yvoìre, où la moraine affleure, et les coupes B1O, 05, 04 qui aboutissent à Evian où les terrains glaciaires ont été bien décrits (Blavoux, 1969). Le glaciaire est pratiquement absent sur le flanc nord de la cuvette lacustre. On peut alors identifier 1 '"horizon $B^{\prime \prime} d^{\prime}$ Houbolt et Jonker comme du glaciaire. 

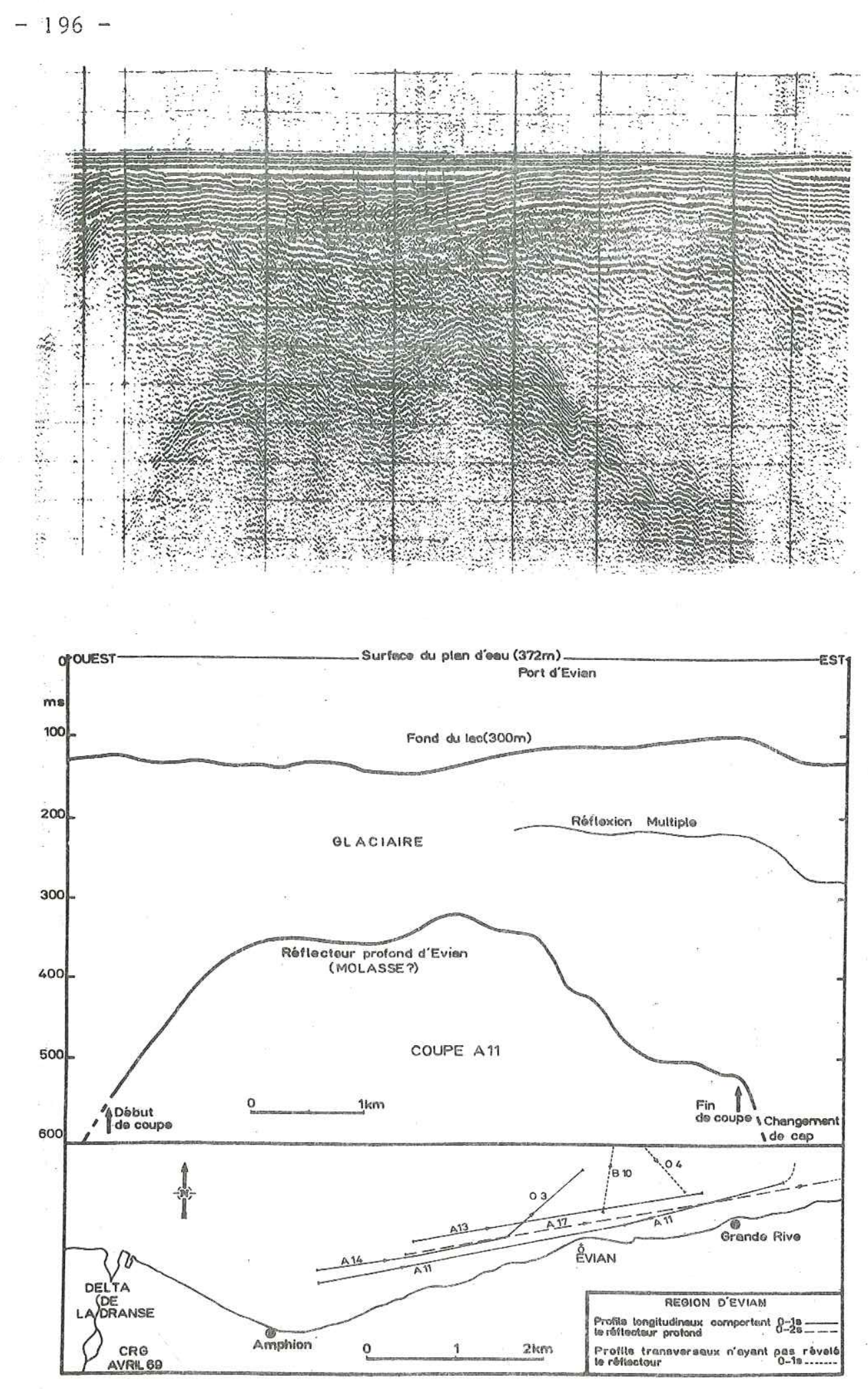

Figure $\mathrm{V}_{4} \quad$ Le Réflecteur Profond d'Evian

découvrir le toit d'un deuxième horizon glaciaire plus profond, 1'épaisseur du premier niveau seraît alors comprîse entre 20 et $100 \mathrm{~ms}$ (temps double de réflexion soît environ de 20 à $100 \mathrm{~m}$ ). Sur le flanc Sud du lac le niveau glaciaire supérieur reposerait alors sur un niveau glacìaìre înférieur (au niveau d'Evian) et directement sur la molasse au centre de la cuvette lacustre.

C'est le toit de ce niveau glaciaire inférieur qui a sans doute été pris par Serruya pour la molasse, l'examen attentif des coupes B13, B11, 04, 05, B10 (fìgure $V_{3}$ ) et B14 quì traversent la rupture de pente entre le flanc Sud et la plaîne centrale, nous pousse à penser que la molasse plonge sous cet horizon glaciaire, on perd ensuite la réflexion sur le toìt de la molasse. Si vraiment le toit de la molasse remontaît au droit d'Evian comme l'ont fait figurer tous les auteurs précédents (surtout Serruya car Houbolt et Jonker ont extrapolé leurs contours dans cette zone) il faudrait reconnaître dans le Réflecteur Profond d'Evian un terrain autochtone sous la molasse et vraìsemblablement faillé.

Le Réflecteur Profond d'Evian (R.P.E.) (figure $V_{4}$ ) a été mis en évidence sur quatre coupes parallèles à la côte et très proches de celle-ci. Ce réflecteur se perd rapidement à plus de 600ms dès qu'on s'éloigne de plus de $800 \mathrm{~m}$ environ de la côte, c'est la raison pour laquelle il avait échappé aux précédentes campagnes. Du côté Ouest on le perd à cause du delta de la Dranse où il est masqué par les mu1tiples, du côté Est nous 1'avons suivi jusqu'au niveau de Tourronde sans le perdre. Notons que le R.P.E. a été repéré également sur la côte à Evian par prospection électrique, il remonte quelquefois très près de 1a surface (quelques dizaines de mètres).

Nous l'avons précédemment attríbué à la molasse, il faut alors penser à une faille importante dans celle-cí d'un rejet de 800 ou $1000 \mathrm{~m}$. Cet accident de direction E-W appartiendrait au grand réseau des failles oligocènes intéressant le substratum cristallin du Jura et 
des Alpes (L. Glangeaud 1947) et îl est probablement à l'origine d'une anomalie gravimétrique de 25 mgals analogue à celle de la vallée du Rhône entre Martigny et le Bouveret (Gonet 1965 et 1969). Il est également possible que le R.P.E. soit le front des Prêalpes du Chablais. Il s'agiraît alors de 1a Nappe Ultrahelvetíque. Cette interprétation est tout à fait possîble, Blavoux (1969) avaît déjà noté que les caractêristîques hydrochîmíques des sources entre Evian et Amphìon étaìent très proches de celles où les affleurements d'Ultrahelvetique sont bien connus (région de St-Gingolph). Nous aurions alors une configuration sous-lacustre analogue à celle de la zone entre Lugrin et le Bouveret où les préalpes plongent abruptement dans le lac. Cela n'exclue évidemment pas 1'existence d'un accident important.

Enfin signalons qu'on distingue jusqu'à cinq réflecteurs secondaires ( $\mathrm{SL}_{1}, \mathrm{SL}_{2}, \mathrm{SL}_{3}, \mathrm{SL}_{4}, \mathrm{SL}_{5}$ ) dans les sédiments lacustres, le dernier étant sans doute recouvert par les dêpôts détritiques du cône sous-lacustre qui fait suite au canyon du Rhône. Bỉen que le canon à air n'ait pas une définition suffisante pour l'étude des sédiments superficiels, ce n'était pas le but de la campagne, notons toutefois sur la coupe B23 un ancien canyon remblayé par la dernière série de sédiments lacustres (fígure $V_{3}$ ). L'orígîne de ces réflexions secondaires est sans doute due à des nîveaux beaucoup plus détritiques. On peut y voir les apports de crues millénaires du Rhône ou encore les restes de phénomènes paroxismiques discontinus comme la rupture de lacs formés par la réunion de deux cônes de déjection dans la vallée du Rhône, ou de celle d'immenses poches d'eau intraglaciaires. Seul des carottages profonds au centre du lac pourraient préciser la nature de ces niveaux.
5.4. - Essai de synthèse des résultats obtenus par sismique continue.

\subsection{1. - Le substratum molassique.}

Nous avons construit (figure $V_{5}$ ) une carte des isochrones du toi.t de la molasse à partîr de celles de Winnock, Serruya et Houbolt ; la topographie de ce toît a été figurée en termes géographiques (barre, vallée, butte, fosse) et non en termes structuraux. Seuls les accidents remarqués par plusieurs auteurs ont été figurés et pour la partie mérìdionale du lac nous n'avons porté que le R.P.E. Enfin nous avons fait figurer les principales données structurales sur la molasse sur la pérîphérîe du lac. On note tout de suite deux directions privilégiées :

- un axe Sud-Ouest Nord-Est quî correspond à l'orientation générale du Petit Lac et aux axes antîclinaux de Messery et de Morges, les fosses d'Yvoire et de Messery seraient alors des surcreusements dans le synclinal molassîque qu'occupe le Petît Lac (Serruya). La barre d'Yvoire-Allaman bien décrite par Winnock et Serruya serait le prolongement de l'anticlinal de Messery auquel correspondrait celui de Morges avec un décalage sans doute dû à un décrochement.

- à partir de St-Sulpice toute la topographie molassique s'oriente Nord-Ouest Sud-Est c'est le cas notamment pour la barre d'Ouchy et la vallée de Villette. Cette dernière est due sans doute à une grande faille qu'il faudrait relier à l'accident de la vallée du Rhône. Enfin notons les deux petítes "vallées" de Morges et de St-Sulpice qu'il faut rapprocher des synclinaux et anticlinaux connus à terre dans cette région.

Le chevauchement de la molasse subalpine sur la molasse autochtone bien visible à Lausanne se poursuit sans doute au niveau de la cuvette lacustre : cet accident est visible sur 1a partie Ouest de 1 a coupe A24 et sur 1a coupe 05 (Evian-St-Prex). I1 aurait plutôt une direction Nord-Est Sud-Ouest et se raccorderait à terre au niveau du Mont de Boissy. La dorsale Pully-Evian dans la molasse décrite par 
Serruya n'a plus alors de raîson d'être.

Enfin sur la côte mérídionale du lac passe le grand ac-

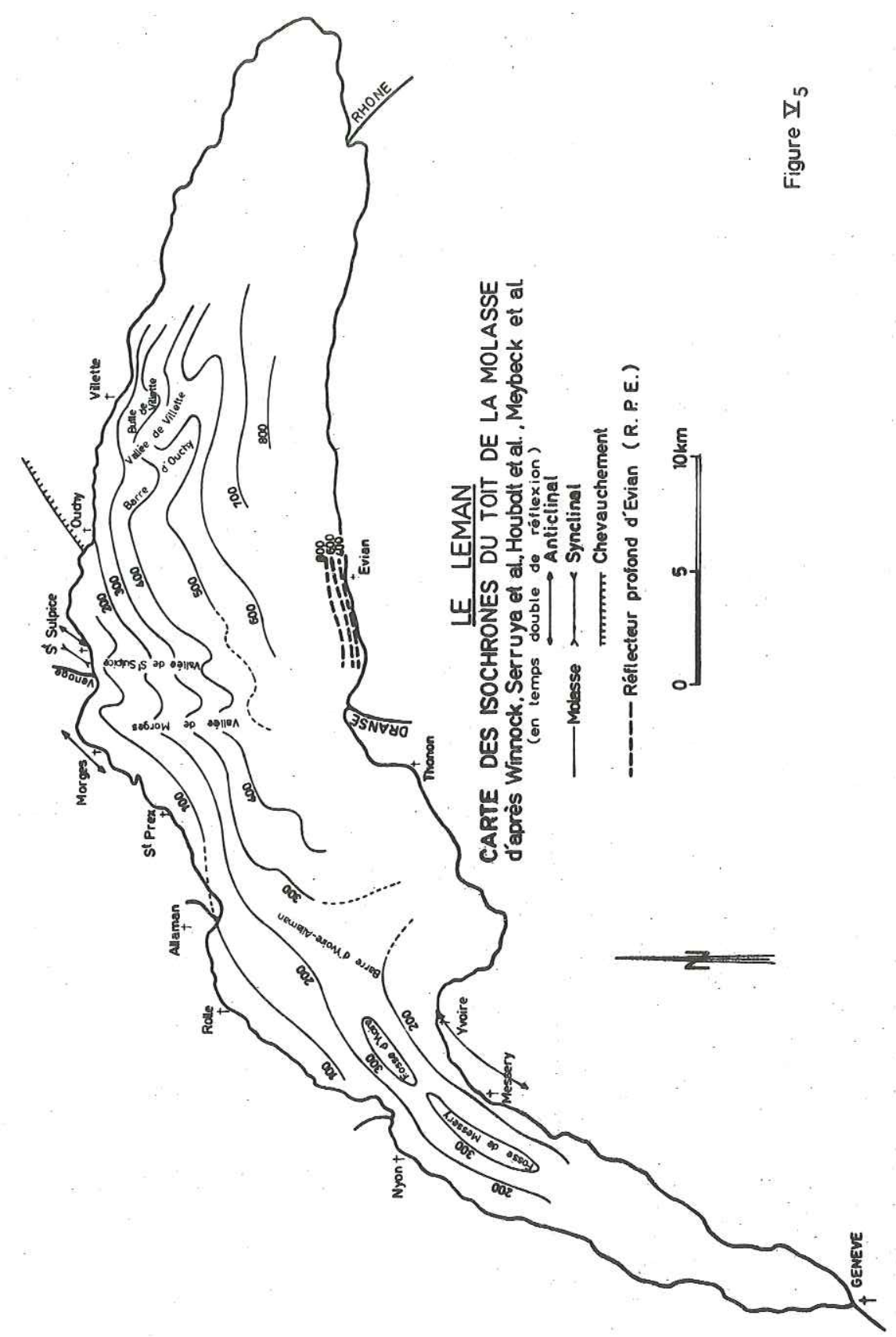

cident d'Evian précédemment décrît. C'est là notre principal point de divergence avec les travaux prédédents. Il n'y aurait pas eu une "grande vallée creusée par 1 'érosîon fluviale" (Serruya) maîs une dépression tectonique due au jeu de grands accîdents d'âge oligocène et qui sont peut être encore actîfs définisissant aìnsi une fosse de subsidence dans $1 \mathrm{a}$ partie orientale de la cuvette lémanique.

\subsection{2. - Epaisseur des niveaux glaciaires.}

Nous avons porté sur la fîgure $V_{6}$ I'épaîsseur du niveau glaciaire supérieur d'après les données d'Houbolt et Jonker 1968 (carte des isochrones de 1'"intervalle A-B") et les nôtres. Nous n'avons pas fait figuré sur cette carte les niveaux glaciaires pour 1e Petit Lac et la baie de Sciez tels qu'ils ont été décrits par Serruya. On remarquera l'absence presque totale de glaciaire sur la côte septentrionale de la cuvette où la morphologie sous-lacustre est directement modelée par la molasse (cas de la butte sous-lacustre de Villette qui serait une île si le niveau du lac baissait de $240 \mathrm{~m}$ ) Sur 1a côte sud par contre le bombement sous-lacustre gênéral à Evian est dû aux dépôts glaciaires. I1 est probable que les dépôts glaciaires atteignent dans cette zone une épaisseur globale de plusieurs centaines de mètres. On notera également d'importantes quantités de glaciaires au centre et à 1'Est de la cuvette.

\subsection{3. - Epaisseur et volume des sédiments lacustres.}

La figure $V_{7}$ montre 1 'épaisseur des sédiments à partir d'une épaisseur minimale de $40 \mathrm{~ms}$ environ $(50 \mathrm{~m})$. Nous n'avons pas pu décrire le phénomène plus finement, le canon à aîr n'ayant pas la même résolution que le sondeur de vase. Il est néanmoins probable que dans toute la zone septentrionale et occidentale de la cuvette l'épaisseur des sédiments soit plutôt de 1'ordre de $20 \mathrm{~m}$ environ (Glangeaud et al, 1964, avec le sondeur de vase). On remarque les épaisseurs considé- 


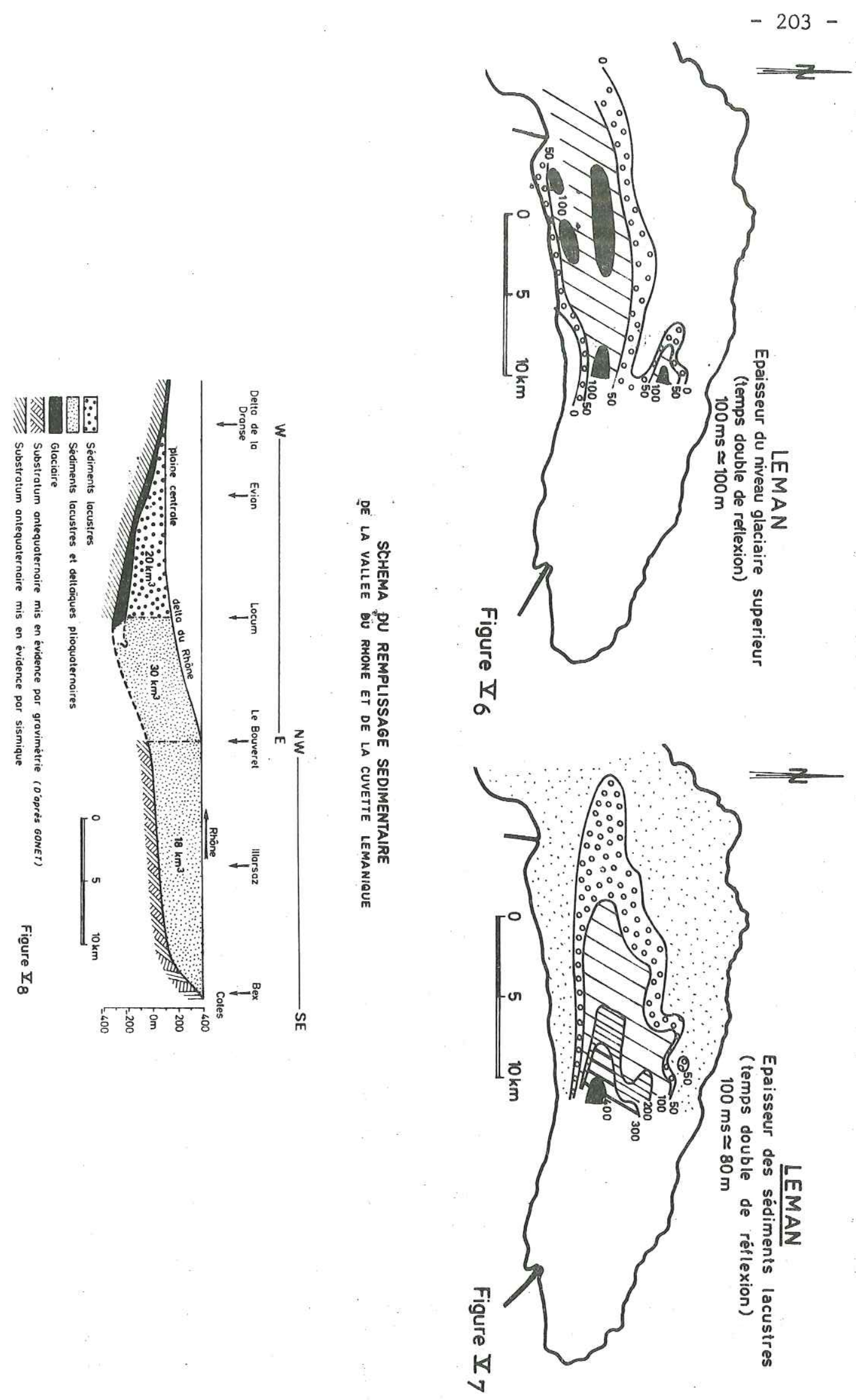

rables (350 m environ) vers Meillerie mais l'absorptîon des échos au-delà de 1a transversale Rivaz-Locum nous oblige vers le Rhône à extrapoler ces contours pour calculer le volume global des sédiments lacustres et deltal̆ques : comme on note dans cette zone à la fois un enfoncement du toit de la molasse et un relèvement des fonds, les dépôts atteîgnent sans doute $500 \mathrm{~m}$ d'êpaisseur. Il faut rapprocher cette estimation aux travaux de Gonet sur 1'épaisseur des alluvions dans la vallée du Rhône : la méthode employée (gravimétrie) ne permettait pas de différencier les dépôts deltaïques des dépôts glaciaires. La carte du bed-rock donne une profondeur de $500 \mathrm{~m}$ entre Villeneuve et 1e Bouveret.

Nous avons portê sur la fígure $V_{8}$ une synthèse des travaux effectués par les deux méthodes. Jusqu'à Locum il est encore possible de différencier les dépôts glaciaires, des sédiments lacustres et deltaïques. Les volumes estimés suivants englobent les deux types de dépôts :

- vallée du Rhône entre Bex et le Bouveret : $18 \mathrm{~km}^{3}$

- delta sous-lacustre du Rhône entre 1e Bouveret et Locum : $30 \mathrm{~km}^{3}$

- sédiments et glaciaire supérieur de la plaine centrale

- sédiments du reste du lac

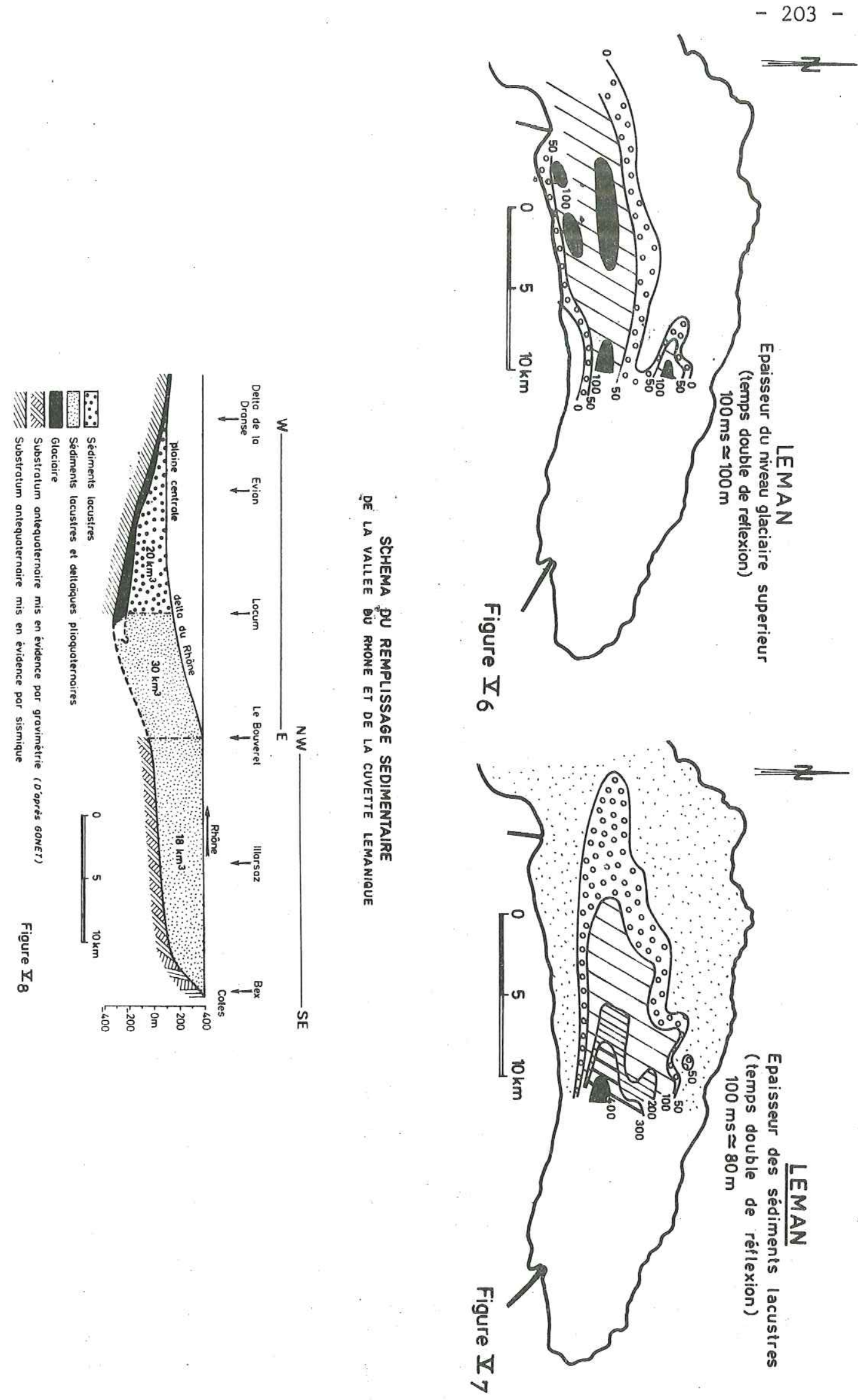

(flanc de 1a cuvette et Petit Lac)

$15 \mathrm{~km}^{3}$

TOTAL

$83 \mathrm{~km}^{3}$

Ces chiffres ne tiennent pas compte ni du delta de la Dranse ni d'une grande épaisseur de glaciaire probable au niveau d'Evian. Nous pensons que sur ce volume global les $1 / 4$ sont à mettre réellement au compte de dépôts de glaciaire supérieur, il 
resterait pour les dépôts lacustres et deltai̊ques postglaciaires un volume de $60 \mathrm{~km}^{3}$ environ. En estimant la masse totale des dépôts, qui peuvent être compactés assez fortement, à $120.10^{9} \mathrm{t}$, et Le retrait des derniers glaciers à 15000 ans (J. Sauvage, 1969), on aurait une moyenne de 8 millions de tonnes/an pour l'ensemble de cette période. Ce chîffre est du même ordre de grandeurs que les données de la période actuelle ( 6 à 7 mìllions de tonnes). Notons la concordance entre les données obtenues à des échelles très différentes (quelques dìzaines d'années et quelques dizaines de milliers d'années). Cependant les estimations faites sur des grandes périodes, quoíque paraîssant beaucoup plus împrécises, nous semblent quand même plus valables car elles intègrent alors les phénomènes très exceptionnels dont nous avons parlé et dont la probabilité d'apparition à l'échelle de temps humaine est très faible.

\section{5. - Comparaison entre la structure du Léman et celle du Bodensee.}

Une foîs de plus nous sommes amenés à comparer les deux grand lacs alpins : dans deux notes G. Müller et R.A. Gees (1968 et 1970) ont décrît les résultats d'une campagne de sismique contìnue effectuée sur le Bodensee. Comme pour le Léman, 3 horizons ont été trouvés : molasse tertiaîre, glaciaire et sédîments lacustres, 1a topographie générale de la cuvette étant due à la molasse. Le travail de Müller basé sur de très nombreuses transversales montre 1'existence d'une profonde vallée dans la molasse remplie de dépôts pleistocènes ( $80 \mathrm{~m}$ au maxîmum) et holocène $(90 \mathrm{~m})$. Ces dépôts augmentent également vers le delta du Rhin où le quaternaire atteint une épaisseur de $336 \mathrm{~m}$. Les similitudes structurales des deux lacs sont grandes, néanmoins Müller et Gees pensent que la cuvette du Bodensee a plutôt une origine glaciaire que tectonique.

\section{6. - Conclusions.}

Nous avons vu tous les progrès que les études de sismique continue ont apportés à la connaissance de la structure et du remplissage. Cette méthode devrait beaucoup se développer pour l'étude des grands lacs. Une foìs de plus la limnologie et l'océanographie se rejoignent. Les prîncîpaux rêsultats acquîs sont les suivants :

- Le Léman a une origine essentiellement tectonique : il y a eu remplissage d'une fosse délimitée par des accidents d'âge oligocène mais qui ont rejoué au Pontien et au Quaternaire (phase néotectonique générale de l'Europe occidentale de L. Glangeaud).

- La morphologie des fonds sous-lacustres est modelée sur celle de la molasse pour toute la partie septentrionale du Grand Lac ainsi que pour le Petit Lac. La topographie du toit de la molasse est alors essentiellement structurale et correspond à des accidents et à des plis bien connus à terre. Sur le flanc méridional de la cuvette les dépôts glaciaires, qui peuvent être très importants, ont déterminé la morphologie des fonds.

- Le remplissage sédimentaire considérable est sans doute lié à une subsidence de la fosse, d'une côte de moins $300 \mathrm{~m}$ par rapport au niveau marin actuel, qui constitue le tréfond de la partie orientale du Léman. Le volume global des dépôts holocènes lacustres et deltaĩques peut être estimé à au moins $60 \mathrm{~km}^{3}$.

Néanmoins il reste de nombreux points à prêciser dans la structure du Léman : le plus important nous semble être l'identification du R.P.E. qui peut se faire soît par sondage sur la côte soît en employant d'autres méthodes de sismique plus puissantes :

(le flexotir). Il faudrait également préciser la topographie de la molasse dans la baie de Sciez et la barre d'Yvoire-Allaman, une très récente campagne entreprise par le B.R.G.M. avec 1a collaboration des Laboratoires de Géologie de Genève et du C.R.G. de Thonon (projet GEOLEM) devrait permettre de lever ces imprécisions. La prospection 
magnétique par magnêtomètre à protons devraît également apporter de nouvelles données, mais seul un sondage profond implanté au large de Meillerie pourra vraiment nous faire connaître la chronologie de tout l'holocène et I'évolution du lac
CHAPITRE W

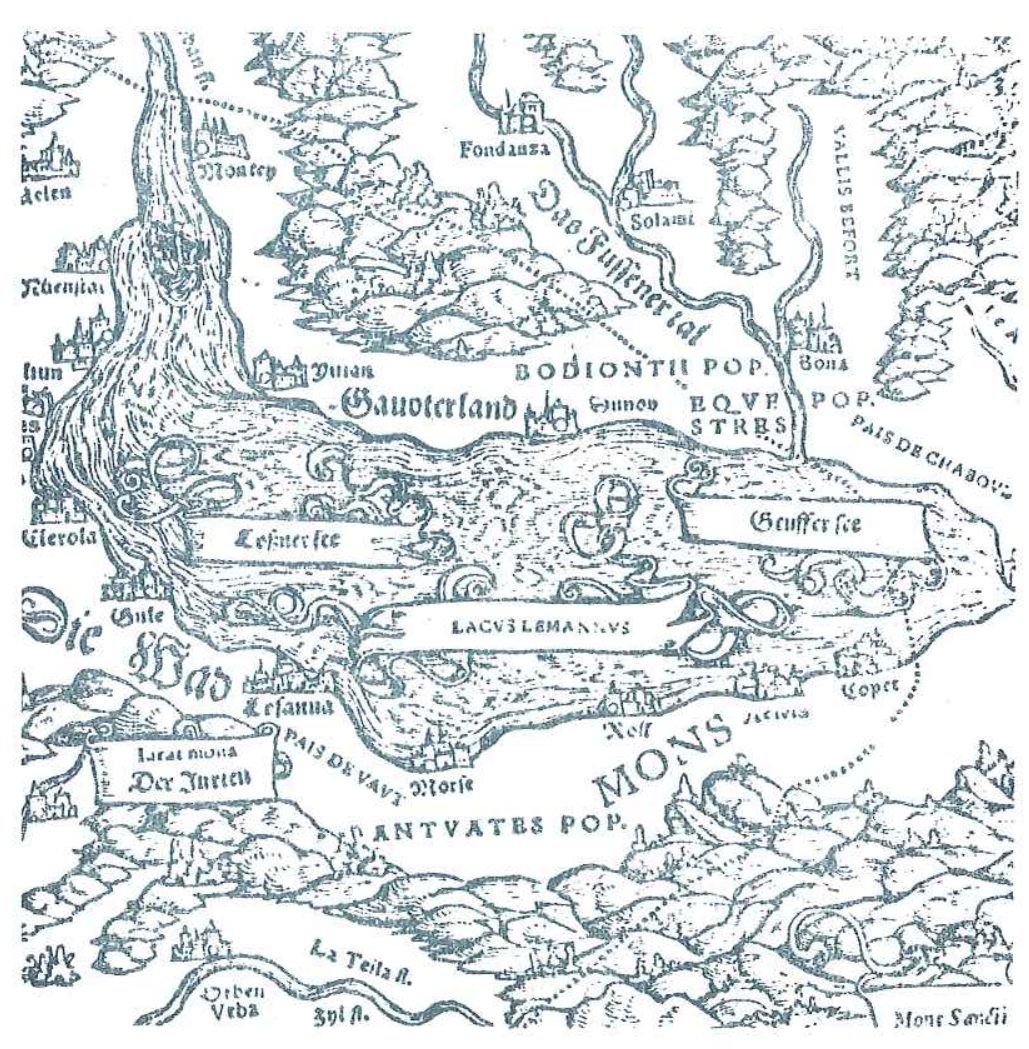

CONCLUSIONS 
Nos conclusions porterons : d'abord sur les résultats proprement dits, puis sur la façon d'y parvenîr, c'est-à-dîre la méthodologie.

\section{1. - Conclusions spécifiques.}

\subsection{1. - L'érosion dans le bassin versant du Léman.}

L'étude des apports en suspensions et des apports dissous, tant au poînt de vue dynamìque que chìmîque, a été effectuée sur les bassins versants du Rhồne $\left(5200 \mathrm{~km}^{2}\right)$ et de la Dranse $\left(550 \mathrm{~km}^{2}\right)$, qui représentent environ $78 \%$ du bassin versant d'alimentation du Léman et $85 \%$ des apports líquîdes. Cette étude a permis de mettre en évidence les faits suivants

- l'érosion globale dans cette partie des Alpes est très forte, de $1^{\prime}$ ordre de $1500 \mathrm{t} / \mathrm{km}^{2} / \mathrm{an}$. La part de 1 'érosion chimique est plus grande pour le bassin de la Dranse (470 t/ $\left./ \mathrm{km}^{2} / \mathrm{an}\right)$ que pour 1e Rhône $\left(210 \mathrm{t} / \mathrm{km}^{2} / \mathrm{an}\right)$. Cette différence est explìcable en première analyse par la nature des terrains essentiellement calcaires pour le Chablais et cristallins pour le Valais, et les débits spécifiques plus élevês de la Dranse. Mais dans l'ensemble l'érosion par dissolution ne représente qu'un tiers de l'érosion totale.

- tous les éléments ne sont pas libérês de la même façon dans les processus d'érosion. La silice provient à $99 \%$ de l'érosion détritique, le calcium et les carbonates proviennent à part 
égale des dissolutions et des désagrégations, tandis que la plus grande partie des composés sulfurés est transportée sous forme dissoute.

- 1'érosion détritique augmente suivant une loî puissance lors des crues $C_{s}=A Q_{1}^{B}$ avec $B>0$. Pendant quelques jours de crue, la rivière peut alors transporter autant de matériaux que pendant une année entière. En même temps l'érosion chimique, quỉ devrait alors être très faible en raison des coefficients de ruissellement élevés, atteint une valeur mînìmale limitée. Si la dissolution et la désagrégation des reliefs sont des phénomènes permanents, le transport des matériaux se fera done principalement lors des crues.

- 1'origine des éléments majeurs dissous a pu être étudiée par les variations des éléments en fonction du débît. Ainsi les concentrations en sulfates, magnésium, et dans une moìndre mesure en calcium et carbonates sont des fonctions dêcroissantes du débit du type : $C_{d}=A Q_{1}^{B}$

avec $C_{d}=$ concentration en éléments dissous, $Q_{1}=$ débit liquide.

$A$ et $B$ coefficients numériques où $B<0$. Ces éléments proviennent donc en grande partie des dissolutions par les eaux souterraines.

- La silice par contre, est en général constante; elle provient essentiellement des altérations superficielles. Les concentrations en chlorures, nitrates sont très îrrégulières et dépendent de la période de l'année, de l'état de lessivage du bassin, etc... Ce comportement suggère une origine artificielle (engrais, pollutions etc...), masquant l'origine naturelle de ces éléments qui se trouve principalement dans les précỉpitations pour le sodium et les chlorures.

\subsection{2. - La sédimentation du Léman.}

- Les sédiments du Léman sont dans leur majeure partie d'origine détritique. Les apports du Rhône, prîncîpalement des particules fỉnes transportées en suspension, conditionnent largement la sédîmentation du Lểman. Il y a deux processus de transport de ces apports : au phénomène général de transport sous forme de suspensions correspondent les vases fines ( 2 à $10 \mu$ ) que 1 'on trouve partout dès qu'on s'éloigne de quelques centaines de mètres des embouchures. Maîs îl existe en plus dans le Léman un phénomène rare dans les lacs : les courants de turbídités. Nous avons pu les mettre en évidence par des études sédimentologiques classiques mais aussi par un traceur naturel des eaux, le trîtium. Ces courants, transportant des matériaux plus grossiers, sont localisés dans les canyons sous-lacustres quî partent du Rhône pour aboutir à la région centrale du Léman. Il se constitue alors un cône résultant du dépôt des sédîments grossiers (turbìites). Plusieurs canyons ont été repérés par des profils d'écho-sondeur, mais il semble qu'actuellement un seul soit régulièrement le siège de courants de turbidités provoqués par les fortes crues du Rhône.

- Dans l'ensemble du lac îl y a précipitation de carbonates d'origine biochîmique au niveau de 1'épilimnion. En général ces particules se redissolvent dans les couches profondes mais dans les zones côtières et surtout dans le Petit Lac cette redissolution est minime, une grande part du sédiment est alors constituée par des carbonates précipités. Dans le Grand Lac cette proportion ne dépasse guère $30 \%$. Dans le Petit Lac par contre, d'une profondeur moyenne de $40 \mathrm{~m}$ elle peut atteîndre $70 \%$. Enfin ill est important de noter que seul 1e carbonate de calcîum est précipité, le magnésium participant très peu à ce processus de précipitation.

- Une première étude des variations comparées des éléments majeurs constîtuant les vases du Léman nous a permis de différencier 
les éléments par groupes :

- les éléments lîés aux aluminosîlicates surtout argileux (illite et chlorite) sont par degré de liaison décroissant : $\mathrm{SiO}_{2}, \mathrm{Al}_{2} \mathrm{O}_{3}, \mathrm{Fe}_{2} \mathrm{O}_{3}, \mathrm{~K}_{2} \mathrm{O}, \mathrm{TiO}_{2}, \mathrm{Na}_{2} \mathrm{O}$ et $\mathrm{TiO}_{2}$.

- une partie de la sîlìce dosée est sous forme de quartz fin et de tests siliceux (diatomées).

- 1e calcium peut provenir des apports détritiques mais dans les zones éloignées du Rhône ou peu profondes, cette origine devient secondaire, devant le calcium d'origine biochimique.

- Enfin le manganèse et le phosphore ont un comportement très à part, l'un par sa forte variabililté, l'autre par sa constance. L'origine de ces variations n'a pas pu être encore trouvée.

\subsection{3. - Composition chimique des eaux du Léman.}

L'étude des eaux du Léman a été poursuivie pendant plus de deux ans en 8 stations hydrologiques réparties sur tout le lac. La comparaison de plus de 700 analyses d'éléments majeurs nous a permis de mettre en évidence les faits suivants :

- Dans son ensemble le lac est caractérisé par une grande stabilité chimique des éléments majeurs : la composition moyenne des eaux est constante d'une année d̀ l'autre et n'a guère varié depuis 100 ans. De plus, à profondeur identique, les eaux ont sensiblement la même composition moyenne annuelle d'un bout à l'autre du lac.

- Si le lac est bien mélangé latéralement, il n'en est pas de même verticalement. Il y a une différence nette entre la couche supérieure $(0-50 \mathrm{~m})$ et les couches profondes qui sont beaucoup plus stables au point de vue chimique :

- La couche supérieure est le siège d'importantes variations saisonnières, essentiellement liées au cycle biologique du plancton, mais aussi aux conditions de température. Ainsì en Septembre l'hypolimnion s'appauvrit fortement en bicarbonates et en calcium par précipitation de $\mathrm{CaCO}_{3}$ due à la présence de $\mathrm{CO}_{2}$ dégagé par le plancton, des pH élevés (jusqu'à 8,6) et des fortes températures $\left(20^{\circ} \mathrm{C}\right)$. Les éléments liês au cycle organîque, comme la silice et les nitrates, présentent des variations très importantes directement opposées au développement du plancton.

- Les couches profondes sont beaucoup plus stables et homogènes au point de vue chimique. On note cependant une augmentation régulière et nette des bicarbonates et du calcium avec la profondeur, elle correspond sans doute dela dissolution des carbonates précipittés. Les sulfates présentent également des valeurs plus élevées dans les couches profondes que dans la couche superrieure mais cette élévation n'est pas régulière jusqu'au fond du lac. La silice, qui est l'élément le plus variable du lac,présente toujours une augmentation dans les couches profondes. Cette élévation provient sans doute de la dissolution du test siliceux des diatomées.

- Un certain nombre d'éléments sont presque invariants dans le Léman, il s'agit en particulier du potassium, du sodium et des chlorures. Le magnésium est presque constant, ce qui souligne un comportement bien différent de celuỉ du calcium.

- A la fin de l'hiver le Léman est presque entièrement homogène au point de vue des éléments dissous. Seule la silice présente encore des concentrations plus élevées à partir de $200 \mathrm{~m}$ de profondeur. Cette homogénéité n'est sans doute qu'apparente, en effet les études isotopiques effectuées par le tritium ont montré que même en hiver le Léman présentait trois couches de comportement 
dynamique différent ( 0 à $50 \mathrm{~m}, 50 \mathrm{~m}$ à $200 \mathrm{~m}$ et $200 \mathrm{~m}$ au fond). L'homogénéité chimique des eaux, variable suivant les hivers, est sans doute réalisée beaucoup plus par diffusion, les températures étant identiques sur toute la colonne d'eau, que par mélange des masses d'eaux.

- L'eutrophisation du Léman s'est traduite par une modification profonde des conditions biologiques, les changements quantitatifs et qualitatifs de la transparence en sont un exemple type. Ce phénomène est surtout lié aux apports considérables depuis 30 ans, d'éléments nutritifs azotés et phosphorés. A part ces éléments, qui représentent moins $\mathrm{d}^{\prime} 1$ \% des sels dissous, 1a composition chimique du Léman est restée remarquablement constante depuis 100 ans, contrairement aux Grand Lacs amérícaìns dont 1'eutrophisation est largemen d'origine industrielle.

\subsection{4. - Remplissage de la cuvette lémanique et bilan du} bassin versant du Léman.

- Devant la variabilité des taux de sédimentation et la difficulté de leur estimation, une étude globale du remplissage de 1a cuvette lémanique a été entreprise par sismique continue. Cette étude a permis de mettre en évidence 1'épaisseur énorme des dêpôts post glaciaires allant jusqu'à $400 \mathrm{~m}$ dans le delta du Rhône. Des cartes des épaisseurs des sédiments, du niveau glaciaire supérieur et du toit de la molasse ont pu ainsi être rểalisées. Les résultats de cette campagne concordent bien avec ce qu'on savait de la structure de la vallée du Rhône. Par contre nous avons pu mettre en évidence pour 1a première fois la présence d'un important accident tectonique Est-Ouest au niveau d'Evian. Ce fait est un argument supplémentaire pour une origine tectonique du Léman. L'étude sismique a aboutit à une estimation des apports détritiques ( $8.10^{6} \mathrm{t} / \mathrm{an}$ ). Cette valeur qui est une moyenne depuis le retrait des derniers glaciers Würmiens concorde avec les estimations basées sur les mesures actuelles.
- Le bilan moyen global du bassin versant du Léman se présente de la façon suivante pour un bassin versant (Léman compris) de $8000 \mathrm{~km}^{2}$ :

TABLEAU VI $\mathrm{I}_{1}:$ BILAN DU LEMAN

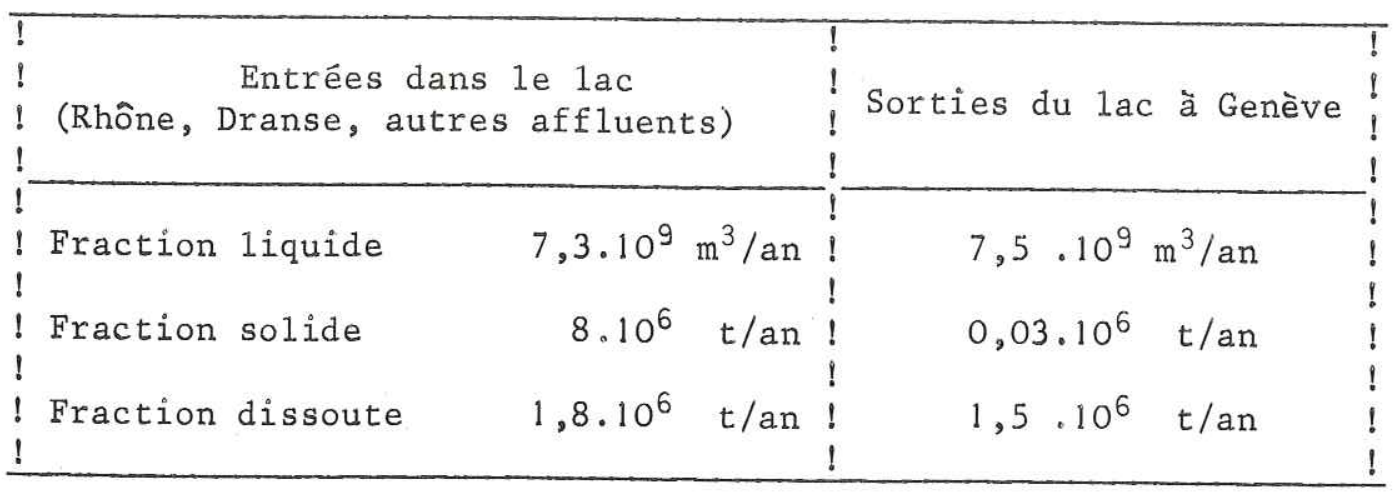

Le déficit du bilan hydrique est dû à la difficulté d'évaluer le ruissellement direct au Lëman. De toutes façons, sì des sources sous-1acustres existent, leur débit doit être faible par rapport aux apports directs.

La précision, encore faible, du bilan des éléments dissous ne permet pas la mise en évidence par cette méthode des précipitations chimiques ou biologiques. Mais ce phénomène a été remarqué par 1'étude de la composition chimique des eaux et des sédiments.

\subsection{5. - Comparaison du Léman et du Bodensee.}

Tout au long de ce travail nous avons remarqué la grande similitude entre le Léman et le Bodensee (Lac de Constance). Nous avons reporté dans le tableau $\mathrm{VI}_{2}$, les données hydrologiques, géochimiques, et sédimentologiques des deux lacs. La similitude des caractères extrinsèques de chaque système limnologique (climat régional, caractères des bassins versants, nature de la dépression 
lacustre...) explique celles des caractères intrinsèques (formes de sédimentation, etc...). Il est important de noter que les deux lacs sont en équilibre géochimique avec leurs bassins-versants. On peut à ce point de vue res ranger dans la catégorie des lacs mìxtes à la foìs silicatés, comme les lacs du Tessin, et calcaîres comme ceux du plateau suisse.

6.2. - Remarques sur les méthodes employées dans l'étude des lacs et l'établissement des bilans.

Nous avons tenté, tout au long de ce travaìl, d'appliquer au système limnologique les principes de la classification scalaire (L. Glangeaud, 1963) notamment en essayant de différencìer les caractères intrinsèques et extrînsèques du système (L. Glangeaud, 1941) et d'identifier à chaque niveau étudiê les phẻnomènes domìnants. $\mathrm{C}^{\prime}$ est dans cet esprit que nous avons rédigé les quelques remarques qui suivent.

\subsection{1. - Etablissement des bilans}

Période d'étude : 1e bilan d'un système limnologique (lac et affluents) doit être soit effectué pendant un temps comparable au temps de renouvellement du système, soit sur une période plus courte en tenant compte des phénomènes caractéristiques mis en évidence.

Précision des bilans : il faut d'abord en considérer tous sositon chimitue du $203 \mathrm{mg} / \mathrm{s}$

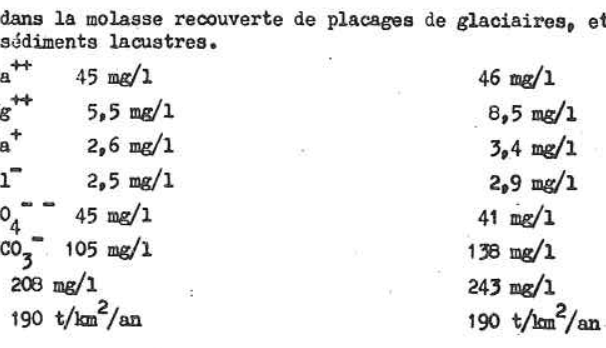

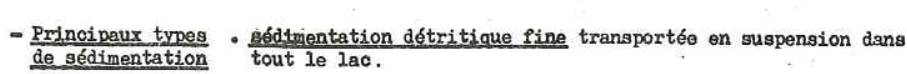

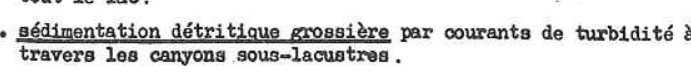

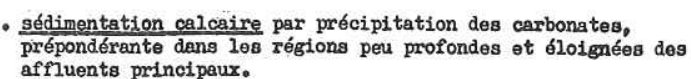
les termes et n'en négliger qu'après avoir déterminé les ordres de grandeur de chacun. Ainsi pour le Lểman nous ne savons rien des retombées sèches et humides, qui sont en général de l'ordre du \% dans les bilans, ni des interactions eau-sédiment : il suffit d'une dissolution suivie d'une diffusion d'une faible partie des minéraux phosphatés pour égaler les apports du Rhône. 
- Il faut déterminer ensuite les lois de variation des éléments dissous ou en suspension et y adapter les modes de calcu1. Nous avons vu que, quand les phénomènes changeaỉent d'éche11e, les lois étaient également différentes.

- Enfin il est essentiel de chiffrer la précision des bilans compte tenu de toutes les erreurs commises : analyses, prêlèvements, débits, mode de calcul des moyennes, apports oubliés ou extrapolés, etc... Ainsi pour notre part nous n'avons pas pu mettre en évidence la précipitation des carbonates : notre bilan des éléments dissous ne doit pas avoîr une meilleure précision que $20 \%$ et pour le bilan sédimentaire la précision est sans doute encore plus faible.

Aussi les conclusions sur 1'évolution des systèmes limnologiques doivent être très prudentes compte tenu des erreurs commises et des variations interannuelles aléatoíres.

\subsection{2. - Emploi des lacs comme modèles réduits.}

Si l'étude des lacs est primordiale pour les ressources en eau de la planète, d'un point de vue strictement scientifique un lac comme le Léman peut être considéré comme une micro-mer intérieure. D'ailleurs ce sont des méthodes venant de l'océanographie : carottages, sismique continue et bientôt peut être le mésoscaphe de J. Piccard, qui ont beaucoup fait progresser notre connaissance du Léman. Inversement le biologiste, le sédimentologue ou le géochimiste peuvent mener à bien sur de tels lacs des études quí seraient beaucoup plus délicates en mer. Un exemple type concerne les courants turbides qui transitent à travers le canyon du Rhône : de tels phênomènes existent partout mais leur étude est beaucoup plus facilement réalísable sur le Léman.

\subsection{3. - Etude de Z'érosion.}

Il est encore très difficile d'extrapoler les valeurs actuelles d'érosion chimique ou détrîtique aux temps géologiques. En effet les phénomènes géologiques comme l'érosion, sont essentiellement discontinus (Glangeaud, Tricart). Ces études auront done une valeur quantitative ponctuelle. Par contre elles ont une valeur qualitative certaine et permettent de déterminer les mécanismes des phénomènes. Pour 1'érosion détrîtique les études de remplissage de cuvette ont des valeurs intégratives et peuvent donc donner des estimations satisfaisantes. Pour l'érosion chìmîque, sî on considère que les lacs sont en général en équiliıbre chìmique avec les apports de leurs bassins versants, il sera possible en étudiant leur composition d'avoir des données moyennes sur l'ensemble du bassin versant. C'est d'ailleurs ce principe qui est appliqué en paléoclimatologie et en paléogéochimie.

\subsection{4. - Etude du système Zimnologique.}

Un lac constitue un milieu récepteur dont les caractères intrinsèques -thermique, morphologie, chimisme, sédimentation, degré de trophie-sont d'abord déterminés par un certain nombre de facteurs extrinsèques régionaux : climat général (température, pluviosité, degré d'ensoleillement,...), nature géographique et géologique du bassin versant, origine géologique et âge de la dépression lacustre. Dans ce milieu récepteur on peut définir cinq ensembles scalaires bien déterminés: l'eau, les électrolytes et gaz dissous, la biomasse, les suspensions minérales et les sédiments. L'étude d'un de ces ensembles n'est possible qu'en considérant toutes ses interactions avec les autres ensembles. C'est ce que nous avons essayê de faire, autant que la bibliographie le permettait, mais de nombreuses données nous ont encore manqué notamment en biologie. Nous avons pu vêrifier cette intrication des phénomènes à chaque chapitre et on comprend la définition de Vallentyne : "un limnologue est un zoologue qui, pendant l'été, étudie les aspects chimiques 
et botaniques de problèmes géologiques sur des lacs facilement accessibles de $15 \mathrm{~m}$ de profondeur, situés au voisinage des universités". Cette boutade montre bien la difficulté d'un travaîl en lỉmnologie.

Comme on ne peut pas dissocier l'étude d'un lac et celle de son bassin versant, à la notion de "lac" nous préférerons done substituer celle de "système limnologique". L'étude de tels systèmes, vu leur complexité, ne pourra être rêalisée que par des équipes "transcisplinaires" (hydrologue, géochimiste, sédimentologue, biologiste, etc...) travaillant sur des problèmes bien déterminés ; parmi les plus importants citons le retournement hivernal et la dynamique des lacsainsi que les interactions vases-eaux. C'est dans cette voie que nous continuons de travailler au C.R.G.

\section{B I B L I O G R A P H I E}

AMBUHL H., 1969, Die neueste Entwicklung der Vỉerwaldstättersees. Verh. Internat, Verein. Lìnol. 17, 219-230.

ARDESTANI H, 1965, Monographie hydrologìque du bassin de la Dranse d'Abondance.

Thèse d'Ingénìeur - Docteur Parìs.

BALAVOINE $P_{n}, 1925$, Variabilité de la composition de I'eau du lac de Genève.

C.R. seanc. Soc. Phys, Hist. Nat. Genève. 42, 2, $61-63$

BALAVOINE P., 1939, Variation de 1a composition de 1'eau du lac de Genève.

C.R. seanc. Soc. Phys. Hist. Nat. Genève 56, 36-38.

BEETON A, Mo, 1965, Eutrophication of the St Laurent Great Lakes, Limno1. Ocean. 10, 2, 240-254, 1965

BERTHOIS L., 1955, Apports sédimentaires en suspension dans la Loire pendant 1'année 1954.

C.R. Ac. Sc. Paris, 241, 814-816.

BERTHOIS L., 1958, Essai d'évaluation du tonnage des matières apportées en solution par les eaux de la Loire pendant I'année 1957.

Rev. Géomorph. Dyn. 9-10, 146-151. 
BERTHOIS L., 1969, Apports en solution par les eaux de la Loire de 1957 à 1968.

C.R. Ac. Sc. Paris, 269, 2072-2075.

BERTHOIS L., BARBIER M., 1953, Evaluation du tonnage des sêdîments en suspension apportés par la Loire pendant I'année 1953.

C.R. Ac. Sc. Paris, 238, 1865-1867.

BETANT A., PERRENOUD G., 1932, Etudes sur la partie occidentale du Lac de Genève. Courants et températures Mem. Soc. Phys. Hist. Nat. Genève 41, 2, 224-293.

BLANC P., DRAY M., 1967, La nappe du delta de Ia Dranse, qualité chimique des eaux en rapport avec son alimentation.

Rev. Institut Pasteur Lyon, 1, 2, 277-295.

BLAVOUX B., 1969, Les sources minérales d'Evian. Houille Blanche, $1,21-29$.

BLAVOUX B., DUSSART B., MANEGLIER H., SALVETTI Cl., 1962, La région du Léman au point de vue hydrométéorologique. Le Globe, 102, 49-68, Genève.

BLAVOUX B., OLIVE Ph., 1966, Premiers résultats sur la teneur en tritium des eaux du Lac Léman.

C.R. Acad.Sc. Paris, 262, 2245-2248,

BURKARD P., 1964-1969, Rapport sur 1'étude des affluents du Léman. Voir : Commission Internationale de protection du Léman.
CANALI L., 1964, Transport de matérîel en solution et en suspension du Pô.

Bu11. AIHS IX, 1, 17-26.

CANALI L。, ALLODI G., 1962, Contríbution à 1'étude sur le transport solide en suspension dans les cours d'eau et sur la dégradation du sol dans le bassîn du Pô. Bu11. AIHS VII, 4, 93-126.

CANALI L., GHERARDELLI L., 1960, Enquête sur les caractéristiques chimiques et physico-chimiques des eaux du Pồa Polesella et recherches des matières dissoutes dans l'eau au moyen d'analyses chimiques quantitatives et spectrographiques semi-quantitatives. Bu11. AIHS N $N^{\circ} \underline{19}, 24-36$.

CARBONNEL J.P., 1965, Sur les cycles de mise en solution du fer et de 1 a silice en milieu tropical.

C.R. Ac. Sc. Parìs, série D, 260, 4035-4038,

COLLET L. Wo, Les lacs 1925, G. Doin. Editeur, Paris, 320p.

COMMISSION INTERNATIONALE POUR LA PROTECTION DES EAUX DU LEMAN ET DU RHONE CONTRE LA POLLUTION. Rapports à la souscommission technique de MM. BURKARD P., LAURENT P., MATTHEY G., MONOD R. et PONGRATZ E.

CORBEL J., 1957, L'érosion chimique des granîts et silicates sous climats chauds.

Rev. Geomorph. Dyn. 1-2, 4-8, 1957. 
CROUZET E., HUBERT P., OLIVE Ph., SIWERTZ E., MARCE A., 1970, Le tritium dans les mesures d'hydrologie de surface. Détermination expérimentale du coefficient de ruisse1lement.

Journal of Hydrology, 11, 217-229.

DAVIS S.N., 1964, Silicain streams and ground water. Am. Journa1 Science 262, 870-891.

DEBYSER J., 1959, Contribution à 1'étude géochimiques des vases marines.

Thèse, Paris.

DELEBECQUE A., 1898, Les lacs français. Béranger Paris $435 \mathrm{p}$.

DIENERT F., 1913, Etude des projets d'adduction d'eau présentés en vue de l'alimentation de Paris. Impr. municipale, Paris.

DUSSART B., 1954, Température et mouvement des eaux dans les lacs. Ann. Stat. Centr. hydrobiol. App1. ㅌ, 5-128.

DUSSART Bo, 1957, Le sondage par ultra-sons et l'étude des lacs. Rev. Géogr. Phys, et Géo1. Dyn. 2ème série, 1, 2, 99-102.

DUSSART B., 1961, Action des eaux courantes sur 1a sédimentation en lac. Verh. Internat. Verein. Limno1. XIV, 440-446.

DUSSART B., 1963, Les grands lacs d'Europe occidentale. Ann. Biol. II, 11-12, 499-572.

DUSSART B., 1966, Limnologie, Gauthier Villars Paris, 677 p.
DURUM W.H., HEIDEL S.G., TISON L.J., 1960, world wide runoff of dissolved solids. A.I.H.S. Ass. Gen. Helsinski. Pub1. 51, 618-628.

F'OREL F.A., 1892-1904, Le Léman.

Monographie limnologique. Tomes I, II et III. F. Rouge. Lausanne.

FOURNIER F., 1960, C1imat et érosion. P.U.F. Paris, 201 pp.

FOURNIER F., 1969, Transports solides effectués par les cours d'eau. Bu11. A.I.H.S. XIV, 3 .

GAGNEBIN E., 1937, Les invasions glaciaires dans le bassin du Léman. Bu11. 1ab. Geo1. Univ. Lausanne, 58, 1-82.

GAZzoLO T., BASSI G., 1960, Contribution à I'étude du degré d'érosion des sols constituant les bassins versants des cours d'eaux italiens.

A.I.H.S.Ass. Gen. Helsinski, pub1. n ${ }^{\circ}$ 53, 112-134,

GIBBS R.J., 1967, The geochemistry of the Amazon river system. I. The factors that control the salinity and the composition and concentration of the suspended solid. Geo1. Soc. Am. Bu11. 78, 1203-1232.

GLANGEAUD L., 1941, Corrélation statistique, classification et hiérarchie des facteurs intervenant dans la formation des sédiments.

GLANGEAUD L., 1963, L'expérience et la recherche opérationnelle dans les Sciences de la Terre et de la Nature. Revue de Synthèse 29-31, 125-170, Paris. 
GLANGEAUD L., 1968, Revue Géogr. Phys. Géol. Dyn. 10, 83.

GLANGEAUD Lin, LEENHARDT O., SERRUYA C., 1964, Structures enregistrées par 1e "mud penetrator" dans les sédiments quaternaires du Léman.

C.R. Ac. Sc., Paris, 258, 4816-4818.

GONET O., 1965, Etude gravimétrique de la plaine du Rhône. Mat. géo1. Suìsse $n^{0} 6$.

GONET O., 1969, Etude gravimétrique du lac Léman à bord du mésoscaphe "Auguste Piccard".

Mat. Geol. Suisse, série géophysique $n^{\circ} 8$.

GORHAM E., DALWAY J.S., 1965, The influence of oxidizing and reducing conditions upon the distribution of some elements in lake sediments.

Limno1. Ocean. 10, 2, 268-279.

GOUGENHEIM A., GENNESSEAUX M., THOMAS A., 1970, Nomenclature des formes de relief océanique.

Cahiers Océanographiques XXII, 8, 769-774.

HEM J.D., 1959, Studies in interpretation of the chemical characteristics of natural water.USGS.

Water Supp1y Paper, 1473, 1-269 pp.

HENSON E. B., VIBBER J.H., 1969, Precipitation into lake Champlain USA, a source of dissolved minera1s. Verh. Internat. Verein. Limno1., 17, 148-153.

HOUBOLT J.J.H.C., JONKER J.B.M., Recent sediments in the eastern part of the lake of Geneva (Lac Léman). Géologie en mijnbouw, 47, 2, 131-148, 1968.
HUBAULT $E_{0}, 1947$, Etudes thermiques, chimiques et biologiques des eaux des lacs de 1'Est de la France.

Ann. Ec. Nat. Eaux et Fôrets, 10, 2, 116-260.

HUBERT P., MARIN E., MEYBECK M., OLIVE Ph., SIWERTZ E., 1969, Aspects hydrologiques, géochimiques et sédimentologíques de 1a crue exceptionnelle du 22 Septembre 1968 de 1 Dranse du Chablais.

Arch. Sc. Genève, 22, 3, 581-604.

HUBERT P., MEYBECK M。, OLIVE Ph., 1970, Etude par le tritium de 1 a dynamique des eaux du Léman.

C.R. Ac. Sc. Paris, série D. 270, 1298-1301.

HUTCHINSON G.E., 1957, A treatise of Limnology. John Wìley and sons edit. New-York. $1015 \mathrm{p}$

INTERNATIONALE GEWASSERSCHUTZ KOMMISSION FUR DEN BODENSEE., 1967, Untersuchungen zur Feststellung der Ursachen für die Verschmutzung des Bodensees. Bericht $n^{0} 6$

JOUKOWSKY E., BUFFLE J.P., 1938, Constitution physique et chimique des sédiments du lac de Genève. Mem. Soc. Phys. Hist. Nat. Genève, 41, 4, 415-456.

JUDSON S., RITTER D.F., 1964, Regional denudation in the United States.

Journal Geoph. Res., 69, 16, 3395-3401

KUENEN P.H., 1950, Marine Geology, (p. 510).

John Wiley and sons New-York. 568 p. 
KUENEN P.H., 1964, Deep sea sands and ancien turbidites. Turbidites,3-33. Bouma and Brouver, E1sevier.

LAL D., 1970, Communication personne1le.

LAURENT P.J., 1966, Modifications biologíques récentes dans quelques lacs subalpins.

Bu11. Ass. Romande Prot. Eaux et de 1'Air (A.R.P.E.A.) $18,47-56$.

LEVINSON A.A., HITCHON B., REEDER S.W., 1969, Major element composition of the Mackensie River et Norman We1ls, NWI Canada.

Geoch. Cosmoch. Acta, 33, 1, 133-137.

LIVINGSTONE D.A., 1963, Chemical composition of rivers and lakes, in Data of geochemistry, 6 th edition, chap. $G$, p. G1-G64, USGS Prof. Paper 440 G.

LOMBARD A., 1939, Influences tectonìques sur le modelê du Léman. Bu11. Ass. geogr. Français, 123, 128-137.

MARTIN J.M., MEYBECK M., HEUZEL M., 1970, A study of the dynamics of suspended matter by means of radioactive tracers : an application to the Gironde estuary. Sedimentology, 14, 27-37.

MEYBECK M., HUBERT P., OLIVE Ph., SIWERTZ E., GLANGEAUD L., 1969, Nouvelles données, obtenues par sismique continue, sur 1a structure de la cuvette lémanique. C.R. Ac. Sc., Paris, série D, 269, 2503-2508.
MEYBECK M., HUBERT P., MARTIN J.M., OLIVE Ph., 1970, Etude par 1e tritium du mélange des eaux en milieu lacustre et estuarien. Application au lac de Genève et à la Gironde. Isotopes Hydrology 1970, 523-541.

Agence Internationale Energie Atomique. Vienne.

MONOD R., 1956, Contribution à 1 'étude des variations de la composition chimique de 1 'eau du lac Léman. Ann. Station Centr. Hydrob. App1., 6, 231-346.

MONOD $R_{0}, 1960$, L'évolution du Léman vue par 1e chîmiste. Revue suisse Hydro1. 22, 1, 242-256.

MONOD R., 1965, Influence des grands froìds (1962-1963) sur l'état sanitaire du Léman.

Bu11. Soc. Vaud. Sc. Nat., 69, 2, 69-82.

MONOD R., 1959-1969, Rapport concernant les examens physiques et chimiques des eaux du Léman.

Voir Commission Internationale de Protection du Léman.

MULLER G., 1964, Zur Hydrochemie von Alpenrheìn und Seerhein. Gas und Wasserfach $105,810-814$.

MULLER G., 1966, a.The new Rhine delta in lake Constance. Deltas in their Geologic framework, 107-124. Houston Geological Society.

MULLER G., 1966, b.Die Sedimentbildung in Bodensee. Die Naturwissenschaften, 10, 237-247.

MULLER G., 1967, Beziehungen zwischen Wasserkörper, Bodensediment und Organismen in Bodensee.

Die Naturwissenschaften, 17, 454-466. 
MULLER G., FORSTNER U., 1968, General relationship between suspended sediment concentration and water discharge in the Alpen rhein and some others rivers.

Nature, 217, 5125, 244-245.

MULLER G., GEES R.A., 1968, Origin of lake Constance Basin. Nature, 217, 836-837.

MULLER G., GEES R.A., 1970, Distríbution and thickness of quaternary sediments in the lake Constance Basin.

Sediment. Geol., 4 .

MULLER G., TIEZ G., 1966, Der Phosphor -Gehalt des Bodensee- Sedimente, seine Beziehung zur Herkunft des Sediment-Materials sowie zum Wasserkörper des Bodensees.

N. Jb. Miner. Abh. 105, 1, 41-62.

PARDE, 1933, Fleuves et rivières. Armand Colin, Paris, 221 pp.

PARDE M., 1952, Sur les valeurs des turbidités spécifiques des rivières. La houille blanche 1952, 2ème journées de 1 'Hydraulique.

PAREJAS E., 1929, L'épaisseur des varves dans le Haut-Lac de Genève. Union gênérale des Rhodaniens. 3ème congrés du Rhône, 207-211. Naville Editeur. Genève.

PORTNER C., 1951, La formation du sédiment calcaire du lac de Neuchate1.

Rev. Suisse Hydrol. XIII, 2, 199-290,

RAMUZ, Composition chimique de 1'eau du Léman. 1957. Bul1. Soc. Vaud. Sc. Nat. 66, 294, 369-375
ROMIEUX J., Les carbonates dans les sédiments du lac de Genève. Thèse Genève 1930. Arch. Sc. Genève 12, 3-4, 202-232.

SAINTIGNON M.F., de, 1970, La sécheresse d'Octobre 1969 dans 1es Alpes Françaises.

Rev. Geogr. Alpine, LVIII, 2, 367-377.

de SAUSSURE H.B., 1779, Voyages dans les Alpes. Neuchate1.

SAUVAGE J., 1969, Etude sporo-pollinìque des sédiments récents du Léman. Docum. Lab. Géo1. Fac. Sc. Lyon, 31, 11-27.

SAYAR M., 1966, Etude géologique, hydrologique, hydrogéologique, climatologique, limnologique, hydrochimique du bassin de 1a Dranse de Morzine. Thèse 3ème cycle, Paris.

SEMENOVICH N.I., 1966, Bottom deposits of lake Ladoga, AIHS Symposium de Garde, publ $\mathrm{n}^{\circ} 70,502-510$.

SERRUYA C., 1969, Les dépôts du lac Léman en relation avec l'évolution du bassin sédimentaire et les caractères du milieu lacustre.

Arch. Sc., Genève, 22, 1, 125-254.

SERRUYA C., LEENHARDT O., GLANGEAUD L., 1966, Structures et évolution post-oligocène de la région du lac Léman. C.R.Acad. Sc. Paris, 259, 1752-1755.

SERRUYA C., VERGNAUD-GRAZZINI C., 1967, Evolution paléoc1imatologique des sédiments du Lac Léman. Arch. Sc. Genève 19, 2, 197-210. 
SHEPARD F.P., DILL R.F., 1966, Submarine canyon and others sea valleys. Rand Mc Na11y. Chicago.

TRICART J., 1962, Les discontinuités dans les phénomènes d'érosion. A.I.H.S. Colloque de Bari. Publ. n ${ }^{\circ}$ 59, 233-243.

VERNET J.P.,1969, Etude pétrographíque des matières en suspension dans le Rhône et ses affluents.

Bu11. Soc. Vaud. Sc. Nat. 70, 5, 215-221.

VIVIER P., ORAND A., 1966, Sur les variations de quelques éléments chimiques dans le lac de Nantua. Mélanges à Maurice Pardé, 717-728. Grenoble.

VIVIER P., SERRUYA S., ORAND A., 1967, Caractères généraux de la po1lution organique du 1 ac de Nantua.

Revue Institut Pasteur Lyon, 1, 3, 411-429.

WAIBEL F., 1962, Das Rheinde1ta în Bodensee, 10. Osterr. Rheìnbauleitung. Bregenz.

WINNOCK E., 1965, Sismique sur le Léman, résultats obtenus avec le sparker.

Bu11. Ver. Schweiz. Petr. Geol. Ing. 32, 82, 39-48,

YOUNG A., 1969, Present rate of 1and erosion.

Nature 224, 851-852.

ZENDER J., 1908, Sur 1a composition chimique de 1'eau et des vases des grands lacs de la Suisse.

Thèse. Université Genève.

\section{I S T E D E S F I GUR.ES}

Pages

\section{CHAPITRE I.}

It Les Grands Lacs pêrialpins.

$I_{2}$ Le Bassin Versant du Léman.

i. 3 Ie Lểman, carte bathymétrique, coupes

bachymétriques et. courbe hypsomé-

txique.

I4 Le Lêman : évolution thermique de

CRG 3 en 1968

i. Carte structurale de la région du Léman

\section{CHAPITE II}

Ii Variation des éléments dissous du Rhône avec je débit

a $\mathrm{HCO}_{3}{ }^{-}$e $\mathrm{Na}^{+}$i $\mathrm{NO}_{3}$

b $\mathrm{Ca}^{++} \quad \mathrm{f} \mathrm{K}^{+}$

c $\mathrm{SO}_{4}^{-{ }^{-}} \mathrm{g} \quad \mathrm{SiO}_{2}$

II2 khône. Corréiations entre les éléments dissous.

$$
\begin{array}{lll}
\text { a } & \mathrm{CI}^{-} & \mathrm{Na}^{+} \\
\text {b } & \mathrm{SO}_{4}{ }^{-}- & \mathrm{Mg}^{++} \\
\text {c } & \mathrm{HCO}_{3}{ }^{++} & \mathrm{Ca}^{++}
\end{array}
$$

II Carte du bassin versant de la Dranse. 
$I_{4}$ Variation des éléments dissous de la Dranse avec le débit.

$\begin{array}{llll}\text { a } & \mathrm{HCO}_{3}^{-} & \text {e } & \mathrm{Na}^{+} \\ \text {b } & \mathrm{Ca}^{++} & \text {f } & \mathrm{SiO}_{2} \\ \text { c } & \mathrm{SO}_{4}{ }^{-}- & \mathrm{g} & \mathrm{Cl}^{-} \\ \text {d } & \mathrm{Mg}^{++} & \mathrm{h} & \mathrm{NO}_{3}^{-}\end{array}$

II $_{5}$

$\mathrm{h} \mathrm{NO}_{3}{ }^{-}$

Dranse. Corrélation entre les éléments

dissous.

a $\mathrm{Cl}^{-}-\mathrm{Na}^{+} \quad \mathrm{C} \mathrm{Mg}^{++}-\mathrm{SO}_{4}{ }^{-}$

b $\mathrm{Ca}^{++}-\mathrm{HCO}_{3}{ }^{-}$d $\mathrm{SiO}_{2}{ }^{-}-\mathrm{NO}_{3}{ }^{-}$

II 6 Hydrogrammes de 1a Dranse et du Rhône.

$\mathrm{II}_{7}$ Variation de la concentration globale du Rhône.

II Variation de la concentration globale de la Dranse.

II Variation de la teneur en sulfates pour le Rhin, le Rhône et la Dranse.

$\mathrm{II}_{10}$ Variation de la concentration globale en fonction du débit pour 6 rivières.

\section{CHAPITRE III}

III $_{1}$ Les stations Hydrologiques sur le Léman

$\mathrm{III}_{2}$ Variation des éléments dissous dans la couche superficielle à CRG 3.

$\mathrm{III}_{3}$ Caractères physicochimiques des eaux à CRG 3

a température

b oxygène

c $\mathrm{pH}$

\section{d transparence}

$\mathrm{III}_{4}$ Evolution des bicarbonates, calcium, sulfates
III5 Evolution des $\mathrm{HCO}_{3}, \mathrm{SO}_{4}$, silice pour la couche superficielle du Lêman.

III6 Variation de la concentration globale du lac。 Couche superficielle.

III7 Variation de la concentration globale moyenne du 1ac. Couche moyenne.

III8 Variation de la concentration globale moyenne du lac. Couche intermédiaire.

III Schéma de la circulation gênérale du Léman.

III $_{0}$ Evolution des teneurs en tritium au centre du Léman.

III $_{11}$ Variation des teneurs en silìce au point CRG 10 .

III 12 Variation des teneurs en tritium à CRG 9 et CRG 21 avant et après la crue de 1a Dranse.

III $_{13}$ Evolution des éléments princîpaux dans le Léman depuis 1808 .

III $_{14}$ Variation comparée de la transparence en 1874 et 1968 .

III 5 Evolution du caractère eutrophe du Léman.

A Transparence

B Oxygène dissout

C Tonnage global des êléments azotés et phosphorés.

CHAPITRE IV

Charge en suspension du Rhône et du Rhìn de 1965 à 1967 .

IV 2 Rhône (Porte de Scex). Relation entre la et silice à CRG 3 . paraison des années 1965 et 1966 . 
IV 3 Rhône (Porte de Scex). Relation entre la charge en suspension $\mathrm{Cs}$ et le débit liquide Q1. Diffêrence entre crues et décrues pour les périodes de hautes eaux des années 1965-1966 et 1967. Dranse du Chablais - Relation charge en suspension - débit liquíde.

$\mathrm{IV}_{5}$ Erosion détritique - érosion chimique.

IV 6 Variation de la concentration en éléments dîssous en fonction du débít spécifique.

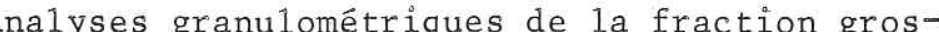
sière.

$\mathrm{IV}_{8}$ Photo de 1'installation de filtration.

$\mathrm{IV}_{9}$ Schêma de I'installation de filtration.

IV 10 Installation de filtration. Schéma d'une cuve

IV 11 Emplacement des prélèvements de sédiments superficiels.

IV $_{12}$ Profils transverses du canyon du Rhône.

IV 13 Profil bathymétrìque St-Gingolph-Vevey.

$\mathrm{IV}_{14}$ Description des carottes LZ 11 et LZ 7.

IV 15 Description des carottes LZ 4 et LZ 8.

$\mathrm{IV}_{16}$ Description des carottes LZ 1 et LZ 10.

IV Teneur en carbonate dans le Léman.

IV Variations des teneurs en silice et en $\mathrm{CaCO}_{3}$ dans les sédiments superficiels.
Véfecteur profond d'Evian : coupes, interprétations et trajets.

$V_{5}$ Carte des isochrones du toit de 1a molasse.

$V_{6} \quad$ Epaisseur du niveau glacỉaire supérieur.

$V_{7} \quad$ Epaisseur des sédiments lacustres.

V8 Schéma du remplissage de la cuvette lémaníque.

Pages

(trajets et appareils utilisés).

$\mathrm{V}_{2}$ Schéma structural de la région lémanique. Trajets des profils sismiques.

$V_{3} \quad$ Profils sismiques et leur interprétation

$\mathrm{V}_{3 \mathrm{a}}$ Coupe $\mathrm{A}_{24}$.

$\mathrm{V}_{3_{\mathrm{b}}}$ Coupe $\mathrm{B}_{10}$.

192

$\mathrm{V}_{3}$ Coupe $B_{23}$ 。 
II $I_{1}$ Comparaison des analyses chimiques des troìs Dranses du Chablais.

$\mathrm{II}_{2}$ Analyses chỉmiques du Rhône à 1 'entrée du Léman.

$\mathrm{II}_{3}$ Bilan des apports dissous du Rhône.

$\mathrm{II}_{4} \quad$ Bîlan des apports dissous de la Dranse.

II 5 Erosion chimique spécifique par éléments, du Rhồne, de la Dranse et du Rhìn alpestre.

III $_{1}$ Coordonnées des stations de prélèvements hydrologiques.

III $_{2}$ Analyses chimiques complètes du Rhône à la sortie du Léman.

$\mathrm{III}_{3}$ Concentrations en $\mathrm{HCO}_{3}{ }^{-}, \mathrm{Ca}^{++}, \mathrm{SO}_{4}{ }^{-}$et silice dissoute. Valeurs extrêmes observées en F'évrier 1968 et Mars 1970 dans 1'ensemble du Léman, Genève excepté.

$\mathrm{III}_{4}$ Valeurs extrêmes de la concentration globale rencontrées dans le Léman de Novembre 1967 à Mars 1970 (sur environ 700 analyses).

III 5 Concentrations en $\mathrm{HCO}_{3}{ }^{-}, \mathrm{Ca}^{++}, \mathrm{SO}_{4}{ }^{-}$et silice dissoute dans la couche superficielle. Evolution des points 3, 4, 6, 9, 21, 22, 23 de Février 1968 à Novembre 1969 .

III 6 Moyennes annuelles par points, par couches des concentrations de 9 éléments analysếs couramment. Année 1968. Moyennes annuelles par points, par couches des concentrations de 9 éléments analysés couramment. Année 1969.

III $_{8} \quad$ Concentration globale en éléments dissous par points et par couches de Février 1968 à Mars 1970 
IIIg Teneurs moyennes en élémentis disssous du Léman en 1968

III10 Teneurs moyennes en éléments dissous du Léman en 1969.

III 1 Anaíyse chimique complète du Lac Léman

III $12_{2}$ Analyse chimique comparée d'un courant turbide.

III 3 Bílan gíobal (en tonnes) du Lẻman pour 1968 par élément, effectué sans tenir compte de la variation de teneur du lac

III $14_{4}$ Erosion spécifique comparée, par éléments, des bassins versants du Léman et du Bodensee.

IV 1 Débits liquides et soildes du Rhône en 1967.

$\mathrm{IV}_{2}$ Erosions spécifiques, chìmiques et dêtrîtiques des rivières alpines comparêes à d'autres rivières.

IV 3 Analyses physiques des sëdiments.

$\mathrm{IV}_{4} \quad$ Analyses chimiques des êléments majeurs des apports du Rhône, de la Dranse, des suspensions et des sédiments du Léman. Valeurs moyennes et extrêmes .

IV 5 Apports moyens annuels en suspension des éléments majeurs au Léman.

IV $_{6}$ Analyses chimiques des éléments majeurs des sédiments superficiels.

IVy Variation dans les vases du Léman des éléments majeurs en fonction de la teneur en silice et en $\mathrm{CO}_{2}$

IV 8 Erosions chimiques et détritiques comparées par éléments.

$\mathrm{VI}_{1} \quad$ Bilan du Léman

$\mathrm{VI}_{2}$ Comparaison hydrologique, sédimentologique et géochimique des systèmes lỉmnologíques du Lểman et du Bodensee.
S O M M A I R E

Pages

INTRODUCTION

CHAPITRE I - CARACTERES GENERAUX DU LEMAN.

1.1. - Données morphométríques.

1.2. - Hydrologie.

1.3. - Bathymétrie

1.4. - Thermique et chimie des eaux.

1.5. - Géologie

1.6. - Climat de la régìon du Léman.

1.7. - Comparaìson du Léman et du Bodensee.

CHAPITRE II - APPORTS DES RIVIERES EN ELEMENTS DISSOUS,

2.1. - Caractères de I'étude chîmique des affluents du Léman.

2.2. - Méthodes d'analyses employées.

2.3. - Variations des éléments dissous dans 1e Rhône .

2.3.1. - Caractères du bassin.

2.3.2. - Corrélation entre la concentration et le débit liquide.

2.3.3. - Corrélation entre les éléments. 
2.4. - Variations des éléments dissous dans la Dranse.

2.4.1. - Caractères du bassin.

2.4.2. - Corrélation entre la concentration et le débit liquide.

2.4.3. - Corrélation entre les éléments. 29

2.4.4. - Etude du chimîsme de certains 32

épisodes hydrologìques.

2.4.5. - Orìgíne géographìque des élêments 35

de la Dranse du Chablais.

2.4.6. - Orỉgine géochimíque des êléments 35 dissous de la Dranse.

2.5. - Bilan des apports au Léman.

2.5.1. - Mode de calcul des bilans.

2.5.2. - Bỉan des apports du Rhône.

2.5.3. - Bilan des apports de la Dranse.

2.6. - Erosion des bassins versants par dissolution. 2.6.1. - Erosion spécifique pour chaque élément 46 2.6.2. - Cas de la silice.

2.6.3. - Conciusion.

CHAPITRE III - COMPOSITION CHIMIQUE DES EAUX DU LEMAN ET BILAN DES ELEMENTS DISSOUS.

A - COMPOSITION CHIMIQUE DES EAUX DU LAC

3.1. - Histoire de l'étude chimique des eaux du
3.2. - Prélèvements et analyses.

3.2.1. - Points de prélèvements du CRG.

3.2.2. - Fréquence des prêlèvements.

3.2.3. - Profondeur des prélèvements.

3.4. - Eléments étudiés.

3.2.5. - Problèmes de méthodologie.

3.3. - Variabilité de la compositîn chîmíque. 3.3.1. - Valeurs extrêmes du Léman.

3.3.2. - Etude physico-chimique et chímique du lac au point CRG 3 .

3.3.2.1. - Caractères physicochìmiques généraux.

3.3.2.2. - Etude des éléments à fortes variations

3.3.2.3. - Elements à faibles variations.

3.3.2.4. - Conclusion de l'étude chimique au point CRG 3

3.3.3. - Etude chimique globale du Léman. $\quad 70$

3.3.3.1. - Variations saisonnières. 70

3.3.3.2. - Variations suivant la 72 profondeur .

3.3.3.3. - Variations suivant les 74 régions du lac.

3.3.4. - Etude des eaux de 1'émissaire à Genève.

3.3.4.1. - Comparaison avec 1e 76 reste du lac.

3.3.4.2. - Teneurs moyennes à I'émissaire.
56

58

59

60

60

64

68

72


3.3.5. - Conclusions de l'étude chîmique globale du Lêman.

3.3.6. - Concentration moyenne annuelle du Léman et variations interannuelles.

3.4. - Problème de la circulation générale du lac et du renouve1lement des eaux.

3.4.1. - Position du problème.

3.4.2. - Etude isotopique par le trîtìum.

3.4.3. - Emploi de la silî́ce comme élément traceur.

3.4.4. - Conciusion.

3.5. - Pénétration du Rhône et de la Dranse dans le Léman.

3.5.1. - Etudes antêrieures.

3.5.2. - Etude chimique générale.

3.5.3. - Etude isotopique par le tritium.

3.6. - Evolution des caractères physico-chimiques du Léman depuis 1870

3.6.1. - Evolution des éléments principaux. 3.6.2. - Evolution du caractère eutrophe.

3.6.2.1. - Transparence.

3.6 .2 .2 . - Oxygène

3.6.3.3. - Eléments nutrîtifs.

3.6.3. - Comparaison du Léman et des grands lacs du St-Laurent.

B - BILAN DES ELEMENTS SOLUBLES DU BASSIN DU LEMAN.

3.7. - Problèmes posếs par 1'établissement du bilan. 3.7.1. - Les termes du bilan.

3.7.2. - Période de calcul du bilan.
3. 8。- Bilan。

3.8.1. - Bilan sans tenir compte de la varìation du lac.

3.8.2. - Bilan en tenant compte de la variation du lac.

3.8.3. - Bilan sur plusieurs années.

3. 9. - Erosion chimique du bassìn versant du Léman.

3.10. - Conclusions générales de l'étude et du bilan chimique des eaux du Léman.

CHAPITRE IV - BILAN SEDIMENTAIRE ET SEDIMENTATION ACTUELLE

\section{DU LEMAN.}

A - LES APPORTS TERRIGENES.

4.1. - Erosion du bassin du Léman.

4.1.1. - Méthodologie.

4.1.1.1. - Mesures de turbìdités. 4.1.1.2. - Méthodes de calculs.

4.1.2. - Apports du Rhône.

4.1.2.1. - Variations saisonnières et interannuelles.

4.1.2.2. - Variation de la charge solide au sein d'une crue.

4.1.2.3. - Calcul des apports du 126 Rhône pour 1967.

4.1.2.4. - Apports solides du Rhône 130 au Léman.

4.1.3. - Apports de la Dranse.

4.1.4. - Effets des crues.

4.1.5. - Comblement du Léman.

\author{
118
}

103

103

104

105

105 107

118

118

118

120

123

124

126

133 
4.1.6. - Comparaison des érosions spécifiques détritíques et chîmiques.

4.1.6.1. - Taux d'érosions actuels des Aipes Centrales.

4.1.6.2. - Comparaison des érosions détrîtiques et chìmiques.

4.1.6.3. - Valeur des érosions mesurées actuellement.

4.2. - Nature physique des apports.

4.2.1. - Mode de prélèvement et méthodes d'ana1yses des sédiments.

4.2.2. - Apports du Rhône.

4.2.3. - Apports de 1a Dranse.

4.3. - Nature chimique des apports.

4.3.1. - Filtration des eaux

4.3.2. - Méthodes d'analyses.

4.3.3. - Analyses chimiques des apports du Rhône et de 1a Dranse.

4.4. - Bilan des apports chimiques en suspensions au Léman.

B - LES DEPOTS RECENTS DU LEMAN。

4.5. - Etude antérieures sur les sédiments du Léman.

4.6. - Modes de prélèvements.

4.7. - Etude sédimentologique du Haut-Lac.

4.7.1. - Morphologie du Haut-Lac.

4.7.2. - Fonctionnement du canyon du Rhône.

4.7.3. - La sédimentation fine dans le Lêman.

4.8. - Etude chimique des sédiments actuels.

4.8.1. - Travaux antérieurs.

4.8.2. - Caractères chimiques des sédiments du Léman
4.8.3. - Précipitation des carbonates.

4.8.4. - Premières conclusions sur la nature chimique des sédiments du Léman.

4. 9. - Sédimentation comparée du Léman et des autres lacs.

10. - Taux de sédimentation du Léman.

C - BILAN SEDIMENTAIRE DU BASSIN VERSANT DU LEMAN ET EROSION SPECIFIQUE DES ELEMENTS MAJEURS.

4.11. - Composition chimique des suspensions lacustres. 176 4.12. - Erosion détritique et chìmique comparée par éléments.

\section{CHAPITRE V - STRUCTURE ET REMPLISSAGE DE LA CUVETTE LEMANIQUE.}

5.1. - Nécessité d'une étude sîsmìque.

5.2. - Situation geologique de la cuvette 1́r anique, éléments apportés par les études sismiques.

5.2.1. - Structure de la région.

5.2.2. - Les horizons rencontrés en sismique continue.

5.2.3. - Problèmes posés par l'identification des horizons.

5.3. - Résultats de la campagne de sismique contỉnue d'Avri1 1969.

5.3.1. - Méthode d'étude. Appareillage.

5.3.2. - Résu1tats.

5.4. - Essai de synthèse des résultats obtenus par sismique continue.

5.4.1. - Le substratum molassique.

5.4.2. - Epaisseur des niveaux glaciaires.

5.4.3. - Epaisseur et volume des sédiments lacustres。 
5.5. - Comparaison entre la structure du Léman et celle du Bodensee.

5.6. - Conclusions

6.1. - Conclusions spécifiques. 6.1.1. - L'érosion dans $1 \mathrm{e}$ bassin versant du Léman.

6.1.2. - La sédimentation du Léman.

6.1.3. - Composition chìmique des eaux du Léman.

6.1.4. - Remplissage de la cuvette lémanique et bilan du bassin versant du Léman.

6.1.5. - Comparaison du Léman et du Bodensee.

6.2. - Remarques sur les méthodes employées dans 1'étude des lacs et l'établissement des bilans. 2.1. - Etablissement des bilans.

6.2.2. - Emploi des lacs comme modèles réduits. 6.2.3. - Etude de 1'érosion.

6.2.4. - Etude du système limnologique.

BIBLIOGRAPHIE.

LISTE DES FIGURES.

LISTE DES TABLEAUX.

\author{
E R R A T A \\ - Dans le tableau $\mathrm{IV}_{3}, \mathrm{p} 183$, lire $\mathrm{LB}_{15 \mathrm{~F}} 320 \mu$ \\ au lieu de $\mathrm{LB}_{15 \mathrm{~S}} 320 \mu$. \\ - Il y a une légère différence pour les érosions \\ chimiques du Rhin alpestre entre les tableaux \\ $\mathrm{IV}_{2}$ (p 181) et $\mathrm{VI}_{2}$ (p 214). Cette différence \\ s'explique par les données bibliographiques \\ contradictoires concernant la surface exacte \\ du bassin versant du Rhin, la valeur la plus \\ probable est de $235 \mathrm{t} / \mathrm{km}^{2} / \mathrm{an}$.
}

